\title{
Employments effects of Vocational Rehabilitation in Germany
}

Citation for published version (APA):

Echarti, N. (2019). Employments effects of Vocational Rehabilitation in Germany: A Quantitative Analysis. [Doctoral Thesis, Maastricht University]. Boekenplan Maastricht. https://doi.org/10.26481/dis.20191121ne

Document status and date:

Published: 21/11/2019

DOI:

10.26481/dis.20191121ne

Document Version:

Publisher's PDF, also known as Version of record

\section{Please check the document version of this publication:}

- A submitted manuscript is the version of the article upon submission and before peer-review. There can be important differences between the submitted version and the official published version of record.

People interested in the research are advised to contact the author for the final version of the publication, or visit the DOI to the publisher's website.

- The final author version and the galley proof are versions of the publication after peer review.

- The final published version features the final layout of the paper including the volume, issue and page numbers.

Link to publication

\footnotetext{
General rights rights.

- You may freely distribute the URL identifying the publication in the public portal. please follow below link for the End User Agreement:

www.umlib.nl/taverne-license

Take down policy

If you believe that this document breaches copyright please contact us at:

repository@maastrichtuniversity.nl

providing details and we will investigate your claim.
}

Copyright and moral rights for the publications made accessible in the public portal are retained by the authors and/or other copyright owners and it is a condition of accessing publications that users recognise and abide by the legal requirements associated with these

- Users may download and print one copy of any publication from the public portal for the purpose of private study or research.

- You may not further distribute the material or use it for any profit-making activity or commercial gain

If the publication is distributed under the terms of Article $25 \mathrm{fa}$ of the Dutch Copyright Act, indicated by the "Taverne" license above, 


\section{Employment Effects of \\ Vocational Rehabilitation}

in Germany

A quantitative analysis

Nicolas Echarti

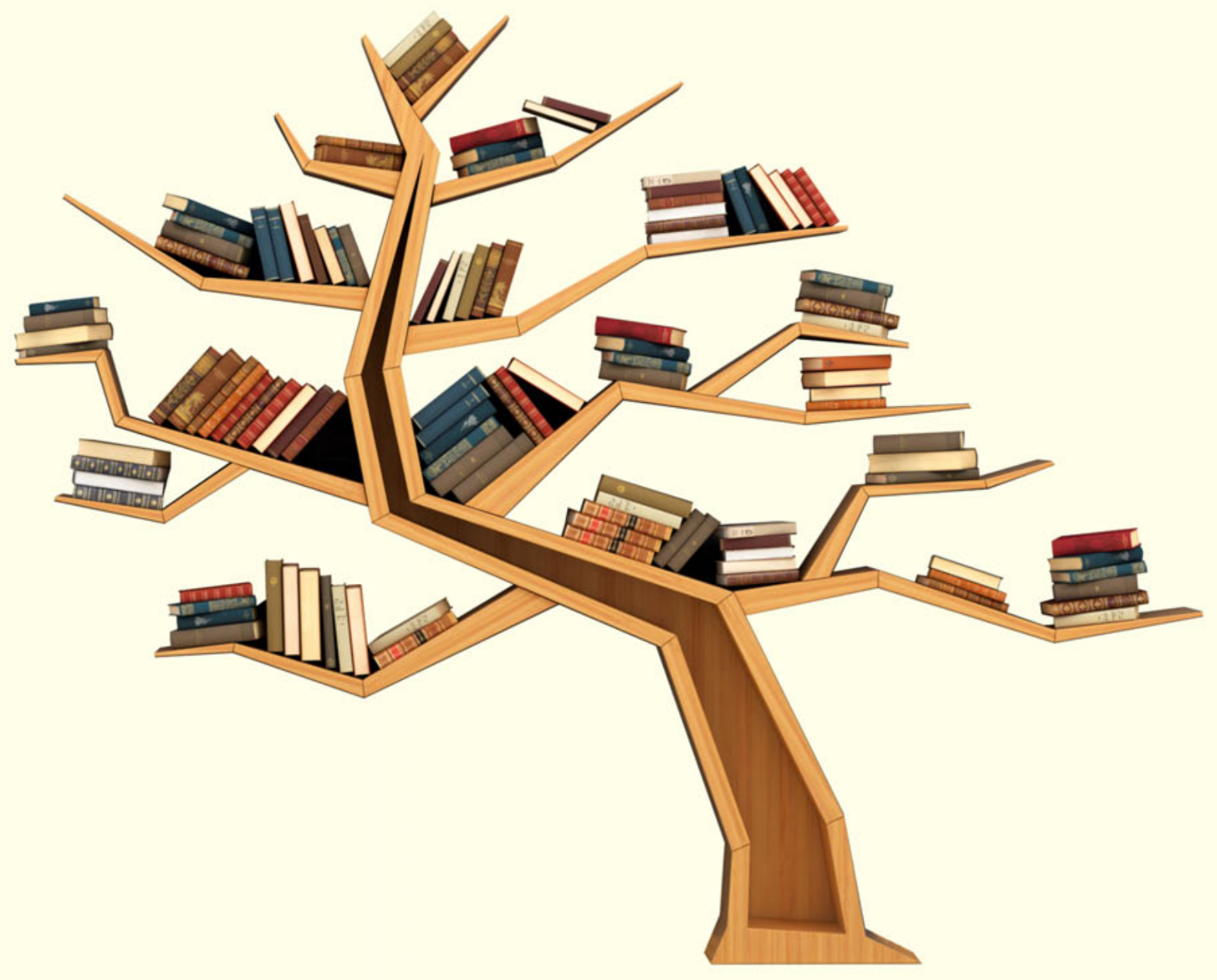





\section{Employment Effects of Vocational Rehabilitation in Germany: A Quantitative Analysis}

Nicolas Echarti 


\section{(C) 2019 Nicolas Echarti}

ISBN 9789086665013

Cover Illustration: urfinguss

Publisher: Boekenplan, Maastricht

All rights reserved. No part of this publication may be reproduced, stored in a retrieval system, or transmitted in any form, or by any means, electronic, mechanical, photocopying, recording, or otherwise, without the prior permission in writing, from the author. 


\title{
Employment Effects of Vocational Rehabilitation in Germany: A Quantitative Analysis
}

\author{
Dissertation
}

to obtain the degree of Doctor at Maastricht University, on the authority of the Rector Magnificus Prof. Dr. Rianne M. Letschert in accordance with the decision of the Board of Deans, to be defended in public on Thursday, 21 November 2019, at 10:00 hours by

Nicolas Echarti 


\section{Supervisors:}

Prof. Dr. Cathal O’Donoghue

Prof. Dr. Esther Schüring, University of Applied Sciences Bonn-Rhein-Sieg

\section{Assessment Committee:}

Prof. Dr. Wim Groot (Chair)

Prof. Dr. Alessio Brown

Prof. Dr. Matthias Bethge, Universität zu Lübeck

Dr. Denisa Sologon, Luxembourg Institute of Socio-Economic Research 


\section{Acknowledgments}

I would like to express my gratitude to my dissertation supervisors Prof. Cathal O'Donoghue and Prof. Esther Schüring, who provided me with continuous academic support throughout the last years. Although doing a part-time PhD means that you and your supervisors are not necessarily in the same place, I always felt that I could reach out anytime and get advice from you. Cathal, thank you especially for deepening my knowledge with regards to carrying out quantitative impact analysis and for pushing me to think hard about the implications of my results. Esther, I sincerely appreciate all the support you have given me since day one, and I am very thankful for all the comments and suggestions you have provided me with during the process of conducting research as well as in the final stages of writing the dissertation. While most of the research for this dissertation was carried out while I was employed at the German Statutory Accident Insurance (DGUV), I would also like to thank Dr. Gregor Kemper and Dr. Joachim Breuer, my main supervisors at DGUV, for giving me the opportunity and flexibility, to combine my work with doing a PhD. In addition, I would like to thank Prof. Karin Hummel and Prof. Edwin Toepler (from the University of Applied Sciences Bonn-Rhein-Sieg) for the helpful comments and suggestions on my early-stage research manuscripts. A special thanks also to Anne Wegner for proofreading many parts of this thesis. Furthermore, I would like to thank Mindel and the entire GPAC staff for the organization of the $\mathrm{PhD}$ program and interesting workshop weeks. I have always felt welcome in Maastricht and certainly have learned a lot during the seminars in the last five years. Moreover, I am very grateful to my family for their consistent support for my education ever since I was young and for the opportunities, they have provided me with. A big thanks also to my friends and fellow classmates for taking my mind of the research from time to time. Finally, I would like to express my appreciation to my partner, Birte, who has provided me with much kindness and love throughout this five-year long research. 


\section{Acronyms}

$\begin{array}{ll}\text { AIC } & \text { Akaike Information Criterion } \\ \text { ARIMA } & \text { autoregressive integrated moving average } \\ \text { ATE } & \text { average treatment effect } \\ \text { ATET } & \text { average treatment effect on the treated } \\ \text { BIC } & \text { Bayesian Information Criterion } \\ \text { CI } & \text { confidence interval } \\ \text { Ctr } & \text { contribution rate } \\ \text { DRV } & \text { Deutsche Rentenversicherung } \\ \text { ICD } & \text { International Classification of Diseases } \\ \text { ICD-10 } & \text { International Classification of Diseases, 10th Revision } \\ \text { ILO } & \text { International Labour Organization } \\ \text { IRR } & \text { internal rate of return } \\ \text { ISSA } & \text { International Social Security Association } \\ \text { IPW } & \text { inverse-probability weighting } \\ \text { IPWRA } & \text { inverse-probability-weighted regression-adjustment } \\ \text { Mlogit } & \text { multinomial logistic regression } \\ \text { MSD } & \text { musculoskeletal disorders } \\ \text { NPV } & \text { net present value } \\ \text { OECD } & \text { Organisation for Economic Co-operation and Development } \\ \text { OLS } & \text { ordinary least squares } \\ \text { PV } & \text { present value } \\ \text { ROI } & \text { return on investment } \\ \text { SD } & \text { standard deviation } \\ \text { SE } & \text { standard error } \\ \text { SGB } & \text { Sozialgesetzbuch } \\ \text { UE } & \text { unemployment } \\ \text { VR } & \text { Wocational rehabilitation } \\ \text { WHO } & \end{array}$




\section{Table of contents}

Acknowledgments ..................................................................................................... v

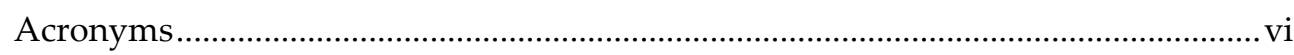

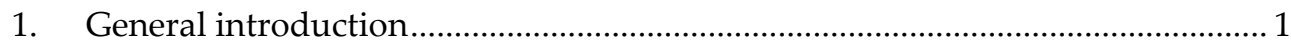

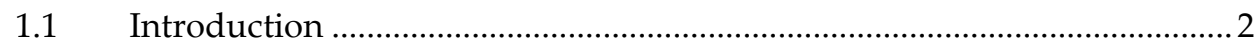

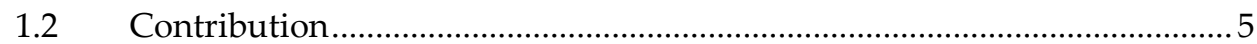

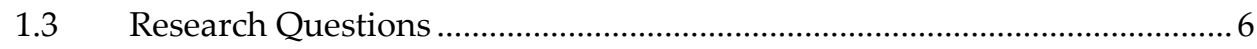

1.4 Study context and research methods.....................................................12

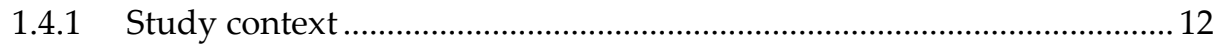

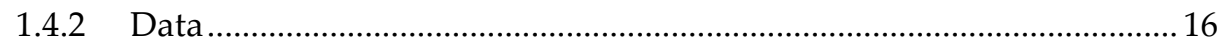

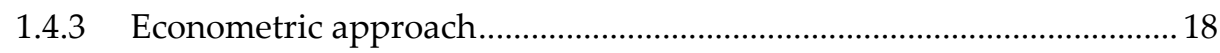

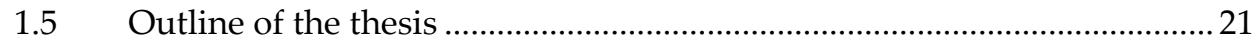

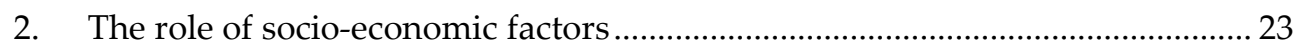

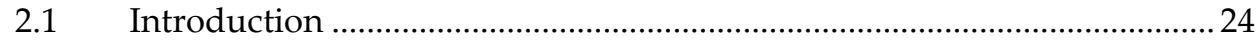

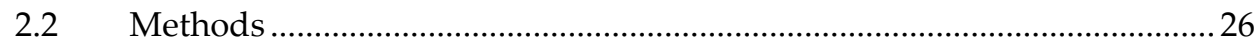

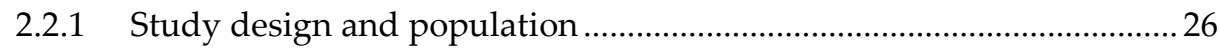

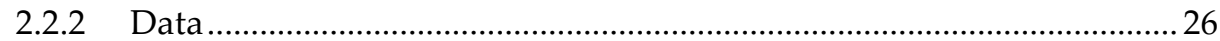

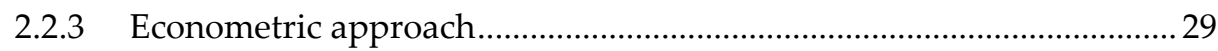

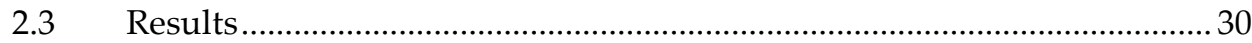

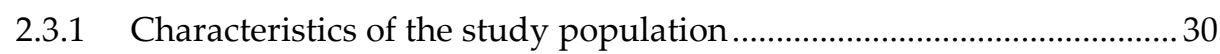

2.3.2 Changes in employment outcomes ……………………………….......... 33

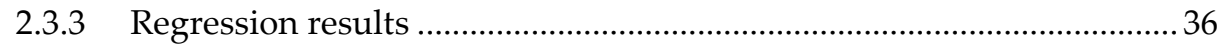

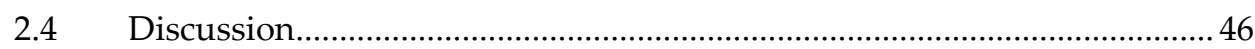

3. The impact of vocational re-training on employment outcomes....................... 49

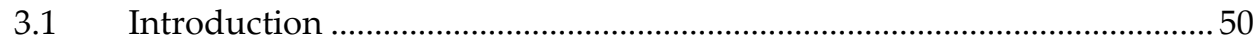

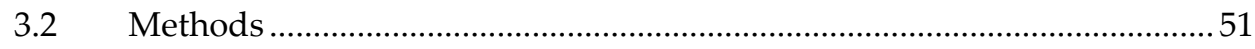

3.2.1 Study design and population ................................................................. 51

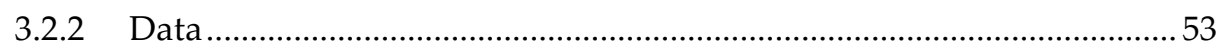

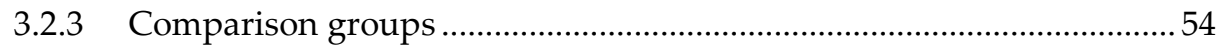




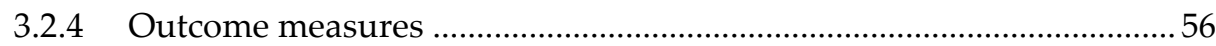

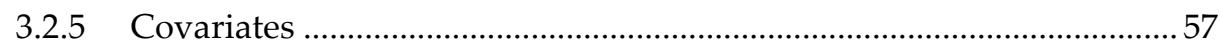

3.2.6 Econometric approach........................................................................... 57

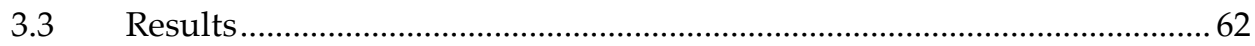

3.3.1 Descriptive characteristics of study participants.................................... 62

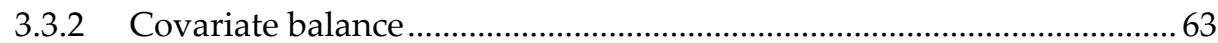

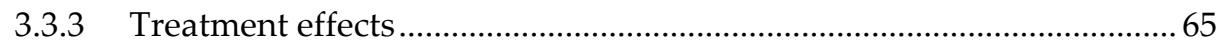

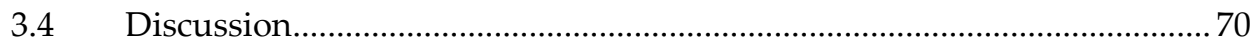

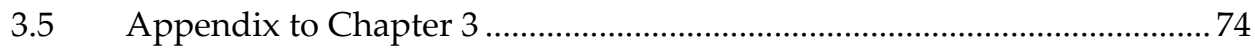

4. One- and two-year re-training in comparison: does the extra year pay off? ... 81

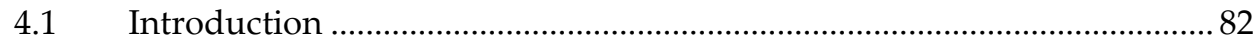

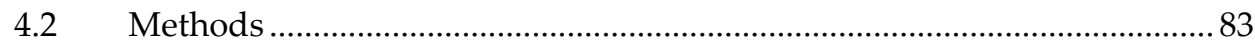

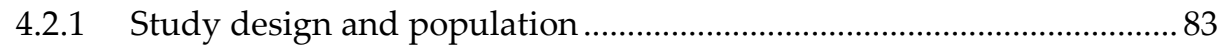

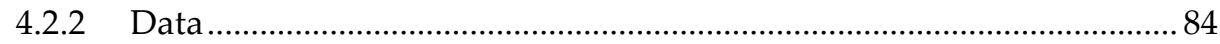

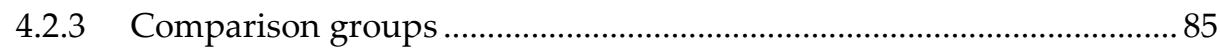

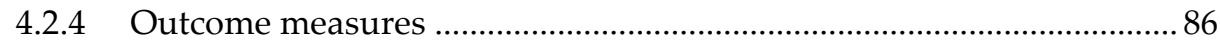

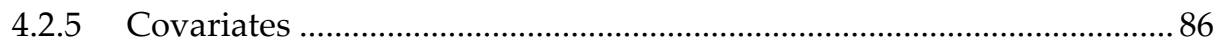

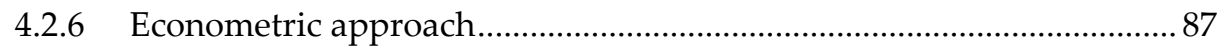

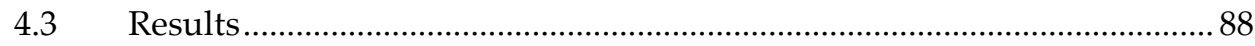

4.3.1 Descriptive characteristics of study participants................................... 88

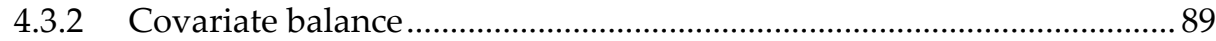

4.3.3 Treatment effects ................................................................................. 92

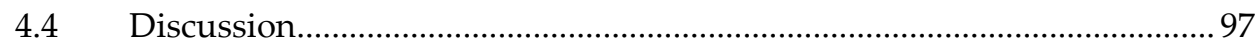

5. The return on vocational re-training: cost-effectiveness from the perspective of

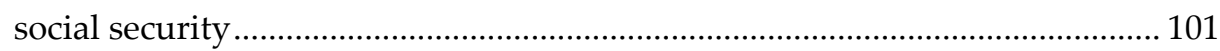

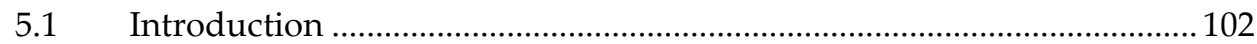

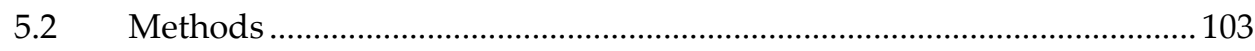

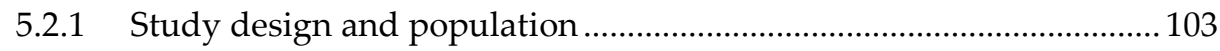

5.2.2 Calculation of benefits to social security............................................... 104

5.2.3 Cost of providing rehabilitation for social security............................... 107 
5.2.4 Cost-effectiveness outcome metrics 109

5.3 Results 110

5.3.1 Benefits of one-and two-year re-training for social security 110

5.3.2 Costs of one-and two-year re-training for social security 112

5.3.3 Cost-effectiveness of one-year and two-year re-training for social security 113

5.3.4 Incremental cost-effectiveness of two-year re-training in comparison

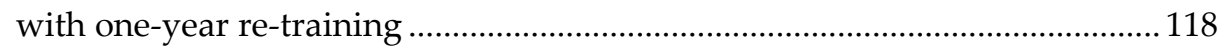

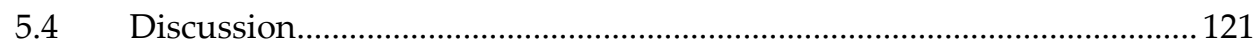

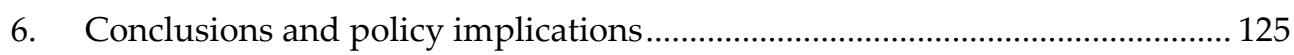

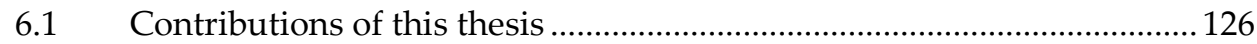

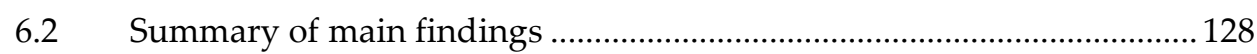

6.3 Policy recommendations .................................................................... 137

6.4 Limitations and areas for future research ...............................................139

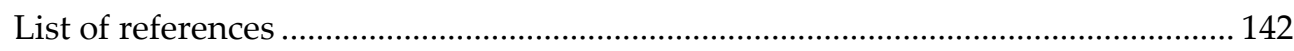

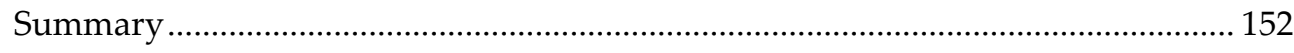

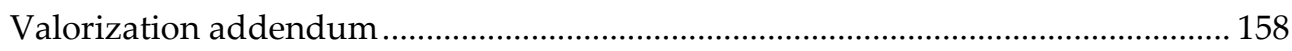

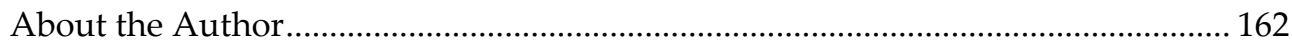




\section{List of Tables}

Table 1: Descriptive Statistics, demographic variables........................................... 31

Table 2: Descriptive Statistics, employment status and last job type ....................... 32

Table 3: Descriptive Statistics, distribution of assigned vocational rehabilitation

measures and main medical diagnosis ....

Table 4: Development of mean labor income, days of employment and days with unemployment benefits

Table 5: OLS regression of socio-economic factors on the before-after difference in income, employment days and short-term unemployment benefits.

Table 6: OLS regression of socio-economic factors on pre-rehabilitation income ... 40

Table 7: OLS regression of socio-economic factors on accumulative employment outcomes over four years after admission into vocational rehabilitation, adjusted for baseline employment outcomes.

Table 8: Stratified OLS regression of socio-economic factors on employment outcomes (by gender).

Table 9: Registered reasons for program dropout...... .55

Table 10: Baseline characteristics of the one-year re-training intervention group, the two-year re-training intervention group and the group of program dropouts. ...... 63

Table 11: Standardized differences before and after re-weighting observations ..... 64 Table 12: Potential outcome means and average treatment effects, one-year and two-year re-training in comparison to no re-training scenario.... 66 Table 13: Potential outcome means without re-training among treated individuals and average treatment effects on the treated, one-year and two-year re-training in comparison to no re-training scenario

Table 14: Comparison of independent variables included in the optimal model under the Bayesian and the Akaike Information Criterion and final model used .. 79

Table 15: Baseline characteristics of re-training participants.

Table 16: Overview propensity scores one- and two-year participants .

Table 17: Standardized differences before and after re-weighting of observations 91 Table 18: Potential outcome means and average treatment effects, participation in two-year vs. one-year re-training

Table 19: Potential outcome means with one-year re-training among treated individuals with a two-year re-training and average treatment effects on the treated.

Table 20: Potential outcome means with two-year re-training among treated individuals with a one-year re-training and average treatment effects on the treated.

Table 21: Annual income difference.

Table 22: Factors used to monetarize the benefits of vocational re-training 107 
Table 23: Factors used to monetarize the costs of vocational re-training 108

Table 24: Measured and extrapolated effects resulting from program completion

Table 25: Benefits to social security for average program completer 111

Table 26: Program duration of one-year and two-year re-training.

Table 27: Type of housing provided during rehabilitation

Table 28: Average costs to social security for re-training measure provided, per program completer.

Table 29: Overview of cost-benefits for the average program completer 114

Table 30: Effect of social discounting on accumulative income forecast. 115

Table 31: Sensitivity analysis: Social discount factor 115

Table 32: Sensitivity analysis: Forecasting model 116

Table 33: Sensitivity analysis: Income tax rate 117

Table 34: Costs of program participation in one- and two-year re-training and additional program costs of two-year re-training, per program participant

Table 35: Measured and extrapolated direct effects resulting from program participation in longer re-training.

Table 36: Additional benefits to social security two-year vs. one-year re-training, per participant

Table 37: Incremental cost-benefit relationship to social security two-year vs. oneyear re-training, per participant. 


\section{List of Figures}

Figure 1 Graphical analysis of before rehabilitation income and gain score.............39

Figure 2 Sampling process: Participants in vocational re-training .............................54

Figure 3 Comparison groups: the impact of vocational re-training .......................... 55

Figure 4 Graphical analysis real income development 2006-2013 in comparison to

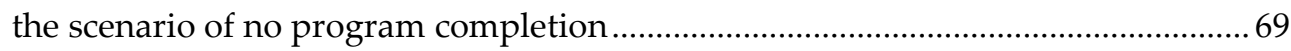

Figure 5 Comparison groups: duration effects of vocational re-training...................85

Figure 6 Overlap plot of propensity scores full re-training vs. partial re-training .. 90 Figure 7 Graphical Analysis of real income development 2006-2013 in comparison

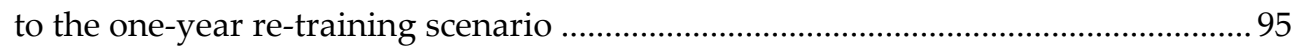

Figure 8 Extrapolation of real wage gains 2013-2030, method of extrapolation: four-

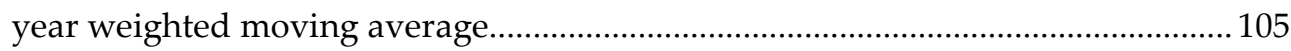

Figure 9 Benefits of re-training to the social security system ................................... 106

Figure 10 Costs of re-training to the social security system...................................... 108 
1. General introduction 


\subsection{Introduction}

Although it has been shown that there are many benefits to hiring people with disabilities (Lindsay, Cagliostro, Albarico, Mortaji, \& Karon, 2018), people with a disability still face considerable economic disadvantages compared to working-age people without disabilities. Disadvantages include lower employment rates, a significantly higher risk of living in poverty, a lower quality of life and reduced social inclusion (OECD, 2010; World Health Organization \& World Bank, 2011).

Since, on average, six percent of the working-age population receive a disability benefit, with this figure rising to more than ten percent in some countries (ISSA, 2012), the situation also constitutes a major public concern: Low employment rates among people with disabilities are in many ways a challenge to economic productivity and the financial stability of social security systems (OECD, 2009). Sickness and disability benefit schemes are costly, and many industrialized countries spend more than twice as much on disability and return-to-work programs compared to unemployment benefit programs (OECD, 2009).

In addition, the outflow from disability benefit schemes is stuck around or even below one percent of those receiving benefits (ISSA, 2012). Countries with an aging workforce and low birth-rates are particularly affected (Duggan \& Imberman, 2008): The prevalence of disabilities increases with age (Berecki-Gisolf, Clay, Collie, \& McClure, 2012) while fewer people are available to produce the contributions needed to be able to finance the growing number of beneficiaries. Finding strategies to enable workers to stay economically active, despite possible health deficiencies, thus, contributes to wider efforts that respond to the challenges facing social security systems and public budgets (ISSA, 2012).

An important goal for public policymakers is to find tools to mitigate the negative impact of disabilities on livelihoods and social security systems, for instance, through implementing comprehensive structural reforms and labor market activation policies and by improving the effectiveness of return-to-work programs (Nikolic, Stanciole, \& Zaydman, 2011). The high individual and societal costs of (work-) disability and long-term sick leave make it crucial to minimize their magnitude and duration.

Work disability is nowadays widely viewed as a public health issue, where the consequences of work disability are considered not only to be following a biomedical causality but also to be influenced by participation restrictions and contextual factors. The consensus is that it is important to understand the dynamic relationship between individual, organizational and societal factors influencing reemployment chances. It is widely accepted that many of the barriers facing people 
with disabilities are, in fact, avoidable and that workers with a disability are far from incompatible with employment in the labor market. Instead, factors such as the absence of access to vocational education and re-training and the lack of financial and other assistance are the significant drivers of labor market exclusion (OECD, 2009; World Health Organization \& World Bank, 2011).

The United Nations Convention on the Rights of Persons with Disabilities (UN General Assembly, 2007) defines the specific actions to be taken by national policymakers to promote the realization of the right to work, including effective access to vocational rehabilitation (VR) and re-training programs. ${ }^{1}$ Vocational retraining programs are usually based on manual or practical activities and are traditionally non-academic but related instead to a specific trade or occupation. Already in 1983, the International Labor Organization (ILO) adopted the Convention C159 on Vocational Rehabilitation and Employment (ILO, 1983). The convention requires member states, given their national circumstances, practices and possibilities, "to formulate, implement and periodically review a national policy on vocational rehabilitation and the employment of persons with disabilities" (ILO, 1983).

Many countries have since adopted vocational rehabilitation programs for people with a disability. However, while there is plenty of research on the impact of labor market programs targeted at regular job seekers (see e.g. Card, Kluve, \& Weber, 2010 for a systematic review), empirical evidence on program effects of vocational rehabilitation programs for people with a disability is scarce. Since the objectives of vocational rehabilitation for people with health problems differ from those of vocational rehabilitation programs for generally unemployed workers, drawing conclusions from non-disabled study populations would be misleading. For people with a disability, it is not a lack of general qualifications that hinders labor market integration but health restrictions that are (or, in the foreseeable future, will become) incompatible with current job duties.

In particular, while systematic evidence exists showing how interventions implemented at the workplace impact employment outcomes (see e.g. Nevala, Pehkonen, Koskela, Ruusuvuori, \& Anttila, 2015; Odeen et al., 2013; van Vilsteren et al., 2015), there is less conclusive evidence on the effects of (out-of-job) re-training

\footnotetext{
1 "States Parties shall safeguard and promote the realization of the right to work, including for those who acquire a disability during the course of employment, by taking appropriate steps, including through legislation, to [...] enable people with a disability to have effective access to general technical and vocational guidance programs, placement services and vocational and continuing training; Promote vocational and professional rehabilitation, job retention and return-to-work programs for people with a disability." (UN General Assembly, 2007, sec. 27)
} 
measures. Some researchers have found a positive effect of re-training on income and employment (Campolieti, Gunderson, \& Smith, 2014; D. Dean, Pepper, Schmidt, \& Stern, 2015; Laaksonen \& Gould, 2015) while others find little or no effects resulting from program participation (Aakvik, 2003; Frölich, Heshmati, \& Lechner, 2004). Interpreting these findings is complicated by differences in study populations, methods used and in the vocational rehabilitation measures analyzed, which may have different mandates, strategies and curricula.

The reviewed evidence indicates, that there is a need for more empirical evidence if the impact of these programs on employment outcomes is to be better understood. So far, there have been only a few attempts to estimate treatment effects of vocational re-training measures, due to researchers struggling with the recruitment of suitable comparison groups and because an appropriate time of follow up is rarely available.

Other uncertainties in the available evidence exist with regards to the influence of patient-level factors on rehabilitation outcomes. From what is known about returning to work after having experienced a disabling injury or disease, the ability to return to meaningful employment depends not only on the quality and type of the rehabilitation measure but also on personal and contextual characteristics (see e.g. Streibelt \& Egner, 2013 for a review). However, much of the available evidence concerning the determining factors is limited to small sample sizes and specific institutional settings. Given the lack of convincing large-scale evidence, there is a need for more research capable of analyzing the effects of influencing factors on the occupational reintegration after participation in vocational rehabilitation. Because participants of vocational rehabilitation are a heterogeneous group of people, knowing the effects of these predictors is also a necessary condition to carry out evaluations on the impact of the programs.

Another gap in knowledge pertains to systematic cost-benefit analyses from the perspective of social-security systems, which, to this date, are largely missing. To assess whether vocational re-training is a worthwhile investment, also from a financial perspective, it is necessary to itemize the services and their resulting costs to be able, consequently, to evaluate them economically. The rise of institutionalized vocational rehabilitation programs in the last decades has both increased the demand for representative empirical evidence on vocational rehabilitation and provided researchers with new opportunities for empirically analyzing the programs' impacts.

Better empirical evidence is needed to enhance our understanding of the conditions that influence the outcomes of vocational rehabilitation programs, to increase their usage and effectiveness and to be able to monitor whether the goals have been met. 


\subsection{Contribution}

This thesis adds to a growing body of literature on the effects of labor market activation programs for people with a disability. While re-affirming the important role of micro-level determinants, the present study provides an extensive example of the individual and fiscal effects that are possible through meaningful vocational rehabilitation measures. The impact analysis presented in this thesis takes a broad perspective and examines the influence of vocational re-training on the number of employment days, income earned, days on unemployment and other social security benefits and on the likelihood of receiving an earnings incapacity pension.

This thesis seeks to make four major contributions to the analysis of the effectiveness of vocational rehabilitation programs for persons with disabilities.

First, it is investigated, how individual-level socio-economic factors influence employment outcomes after participation in vocational rehabilitation in Germany. The analysis includes identifying which factors positively affect re-employment chances and which socio-economic characteristics tend to act as barriers to successful work reintegration. The analysis of socio-economic determinants also serves as a starting point for subsequent impact analysis since to know the effect of socioeconomic factors on employment outcomes is a necessary condition for validly examining an interventions' impact.

Second, while taking the effect of confounding variables into account, it is shown, to what extent completing a one-year or two-year vocational re-training, impacts employment outcomes and to what extent the programs reduce the likelihood of receiving an earnings incapacity pension. In order to investigate the employment effects of vocational re-training measures in Germany, a series of quasi-experiments are carried out. While the selection of potential controls is one of the key challenges to estimate the treatment effects, advanced statistical measures to handle confounding are applied in order to establish comparability between comparison groups. In the absence of randomization, an applicant-based comparison group (program dropouts) is used to compare outcomes with and without completion of a vocational re-training measure. This approach, so far, has been widely underutilized in vocational rehabilitation research. The estimation of treatment effects provides policymakers with detailed information about the interventions' abilities to improve the individuals' employment statuses and show to what extent these improvements are sustained over-time. In addition to its empirical relevance, the research contributes to the methodological debate on how to estimate the impact of training programs from observational data using quasi-experiments and (applicant-based) internal comparison groups. 
Third, the effects of two-year and one-year vocational re-training programs are directly compared to examine the differential impact of these two different forms of re-training. In the light of political initiatives to strengthen horizontal training (qualification for additional tasks), one-year re-training measures (partial qualifications) are becoming increasingly popular as an alternative to the more involved (and more costly) two-year re-training programs (full qualifications). Whereas a recent evaluation of the differential impact of partial and full re-training measures suggests that in the short to medium-term the less involved, one-year programs provide similar employment effects to the traditional, more involved, two-year programs, the long-term effects are unclear. Using an appropriate time of follow up, this thesis re-assesses the question of duration effects of re-training by showing to what extent the long-term effects of one-year and two-year re-training differ.

Lastly, the cost-effectiveness and efficiency of re-training are evaluated from the social security providers' perspective. Relating the benefits to the costs of providing the programs, financial balance sheets are used to illustrate the economic potential of meaningful vocational rehabilitation, showing to what extent the measures can be considered cost-effective. While taking a long-term perspective, it is shown after how many years the programs break even and how large the potential economic returns to the social security system are.

\subsection{Research Questions}

This thesis seeks to answer four main research questions:

1. To what extent do individual socio-economic factors influence return-towork outcomes after participation in vocational rehabilitation?

2. To what extent do vocational re-training programs impact employment outcomes and influence dependency on social security benefits?

3. Are there significant differences between the effects of one- and two-year retraining programs with regards to income earned, employment days and social security benefit receipt?

4. What are the financial returns for providers of the re-training programs: are the measures cost-effective?

In the following section, each research question is developed, first by detailing the importance and prior evidence on the issue and continuing with the contributions this thesis seeks to make. 


\section{To what extent do individual socio-economic factors influence return-to-work outcomes after participation in vocational rehabilitation?}

Since participants of vocational rehabilitation are a heterogeneous group of people, with different social and occupational backgrounds and different health situations, barriers to work reintegration can only be assessed with reference to the individual situation of the participants (Hoffmann, 2004). In a recent study, White et al. (2015) "find consistent evidence [...] for increased risk of work disability in situations where workers have lower education, older age, emotional distress [...] and greater sick leave history." A further recent study on the effects of individual and work factors on work participation trajectories before and after participation in vocational rehabilitation comes to similar conclusions: "Work participation trajectories around vocational rehabilitation are determined by various individual and work-related factors" (Leinonen, Solovieva, Husgafvel-Pursiainen, Laaksonen, \& Viikari-Juntura, 2019).

These two results do not come as a surprise since the complex relationship between demographic and work factors in relation to employment outcomes has been evident in rehabilitation and disability research for quite some time. In particular, age and return-to-work outcomes are negatively correlated (Blackwell, Leierer, Haupt, Kampitsis, \& Wolfson, 2004; Cancelliere et al., 2016; Dodoo-Schittko et al., 2017; Valentin et al., 2016; Van Muijen et al., 2013). A possible explanation is that older workers are less likely to adapt to new situations and have poorer labor market chances than younger applicants. Moreover, many older workers have less of an incentive to return to work as they may be only a few years away from retirement.

Empirical results on the effects of gender are consistent, demonstrating that males exhibit better return-to-work outcomes than females, everything else being equal (Cancelliere et al., 2016; Cornelius, Van Der Klink, Groothoff, \& Brouwer, 2011; Feuerstein, Berkowitz, Haufler, Lopez, \& Huang, 2001; Giesen J. \& Cavenaugh Brenda, 2013; Saltychev, Tenovuo, \& Laimi, 2013; Stergiou-Kita, Mansfield, \& Sokoloff, 2016). A frequently made argument here is that the lower performance of females with regards to labor market reintegration might be the result of a malecentered work environment, of the invisible "glass ceiling" or of greater physical stress and time demands outside the working place.

Over and above age and gender, other factors have been shown to play a role in rehabilitation. For the marital status of the rehabilitants, the majority of findings indicate that married individuals are more likely to return to work and that divorced women are particularly vulnerable to staying out of work. (Kreutzer et al., 2003; Selander, Marnetoft, Bergroth, \& Ekholm, 2002; Street \& Lacey, 2015; Yasuda, Wehman, Targett, Cifu, \& West, 2002). While not all authors agree on the causes, the 
positive effect of being married on rehabilitation outcomes are thought to be partially attributed to the social support received through marriage. Singles are at a greater risk of leaving the labor market permanently.

Various studies have also detected that education is a useful predictor of return-towork outcomes (Blackwell et al., 2004; Cancelliere et al., 2016; Giesen J. \& Cavenaugh Brenda, 2013). Throughout the studies analyzed in that research, the likelihood of successfully returning to work increases with additional education. The results suggest that the improved outcomes for higher-educated individuals may be the result of greater adaptability with regards to coping with the health effects of disabilities, as well as with regards to possible changes in occupational activities.

Regarding the rehabilitants' work and professional lives, the individual work history has been found to affect employment outcomes significantly. It includes past income and employment developments as well as employment or job type related indicators (see for example Adams \& Williams, 2003; Cornelius et al., 2011; Giesen J. \& Cavenaugh Brenda, 2013; Ottomanelli \& Lind, 2009). Blue-collar and manual labor is usually associated with reduced employment outcomes. Moreover, a supportive work environment and the status of employer-employee relations have been found to significantly affect return-to-work rates (Franche et al., 2005; A. Muijzer, Groothoff, Geertzen, \& Brouwer, 2011). Other, more difficult-to-measure characteristics affecting employment results are, for example, the individual motivation for work, intelligence, determination, commitment or the ability to cope with stress (Gericke, 2010).

Taken together, from what is known about returning to work after having experienced a disabling injury or disease, the ability to return to meaningful employment depends not only on the quality and type of the rehabilitation measure but also on personal and contextual characteristics. According to a recent review on studies that examine the drivers of occupational reintegration after vocational education and training (Streibelt \& Egner, 2012b), there is some evidence that this is also true with regards to the employment outcomes after participation in vocational rehabilitation. However, the authors point to the need for further studies to confirm earlier results and to clarify known uncertainties in the available evidence. So far, much of the available evidence concerning the determining factors is limited to small sample sizes and specific institutional settings.

This thesis re-assesses the question of the direction and size of person- and occupation-related factors with regards to employment outcomes after participation in vocational rehabilitation in Germany. One feature of the data used is that it allows outcomes to be compared across different treatments and health states. The data 
includes representative longitudinal employment records of the German pension insurance fund from more than 19,000 participants of vocational rehabilitation between 2007-2009. In the analysis, rehabilitants are compared to each other to determine the extent to which differences in employment outcomes can be linked to the participants' socio-economic backgrounds. By drawing comparisons across rehabilitants, this analysis contributes empirically to a growing body of literature on individual-level facilitators and barriers to return-to-work.

\section{To what extent do vocational re-training programs impact employment outcomes and influence dependency on social security benefits?}

While an impressive amount of literature has shown that individual wages increase with general education (for a review, see Card, 1999) and that re-training for the unemployed improves the employment situation (Card et al., 2010), whether this is also true for vocational re-training measures for people with a disability is unclear. So far there is only limited evidence on the effectiveness of vocational rehabilitation for people with a disability.

Although a plenitude of systematic evidence shows how interventions implemented at the workplace impact employment outcomes (Cullen et al., 2018; Franche et al., 2005; Furlan et al., 2012; Hoosain, de Klerk, \& Burger, 2019; McDowell \& Fossey, 2015; Nevala et al., 2015; Odeen et al., 2013; van Vilsteren et al., 2015), there is less conclusive evidence on the effects of (out-of-job) re-training measures: Some recent empirical studies indicate a positive effect of re-training on income and employment (Campolieti et al., 2014; D. Dean et al., 2015; Laaksonen \& Gould, 2015) while other authors find little or no effects resulting from program participation (Aakvik, 2003; Frölich et al., 2004). Interpreting these findings is complicated by differences in study populations, methods used and in the vocational rehabilitation measures analyzed, which may have different mandates, strategies and curricula.

A common problem when analyzing vocational re-training measures is that researchers generally struggle to recruit study participants, which may limit the types and appropriateness of methods being used (Lysaght, Kranenburg, Armstrong, \& Krupa, 2016). Treatment allocation is generally considered to be nonrandom and dependent on the rehabilitants' individual situations. Due to their individual health situation, people with a disability are a heterogeneous group, making it difficult to find suitable comparison groups for program evaluation (Sears, Rolle, Schulman, \& Wickizer, 2014). Individuals who are deemed eligible to participate in rehabilitation measures usually cannot be denied access to the services. The challenge in evaluating the impact of an intervention is thus to obtain 
a credible estimate on the counterfactual scenario: What would be the outcome had the re-training participants not completed the measures?

In this thesis, rich administrative data about Germany is used as a case study to provide new and unique evidence on the effects of vocational re-training on longterm employment outcomes. While adjusting the results for measured confounders, the research shows to what extent completion of a re-training program influences subsequent income and employment development. Additionally, the influence of the re-training programs on the number of days with unemployment and social security benefits and with regards to the uptake of a pension due to a limited earnings capacity is evaluated.

In the absence of a natural control group, an applicant-based (internal) control group is utilized to estimate the program impact. By drawing a comparison with a group of similar program dropouts, this analysis also adds to a methodological debate on how to estimate program effects of re-training measures from observational data. The estimated treatment effects described in this thesis provide policymakers with detailed information about interventions' abilities to improve an individual's employment status and show to what extent these improvements are sustained over time.

\section{Are there significant differences between the effects of one- and two-year re- training programs with regards to income earned, employment days and social security benefit receipt?}

Traditionally, German vocational re-training programs have focused on long-term re-training measures that are often completed by formal examinations. Following criticism with regards to lock-in effects, there has been an increase in the usage of so-called partial re-training measures that usually can be completed within a year. Due to political initiatives that have the aim to strengthen the role of horizontal training, partial qualifications are becoming increasingly popular as an alternative to the more involved full re-training.

A recent evaluation of the differential impact of partial and full re-training measures (Bethge \& Streibelt, 2015) suggests that the, shorter, partial programs provide similar employment effects to the traditional, more involved, full programs. Using four years of follow-up data, the authors showed that, for the matched rehabilitants, partial qualifications led to at least comparable integration results compared to qualifications from the longer full re-training programs. The results are linked to the earlier findings from Biewen \& Waller (2007), who find that short-term programs are surprisingly effective when compared to the traditional and more expensive longer-term degree programs; a finding similar to that of Fitzenberger \& Völter 
(2007), who examined the comparative effectiveness of public-sponsored training programs of the German federal employment office. ${ }^{2}$

However, compared to the duration of the measures studied, the post-treatment observation period of previously published studies is too short to examine the question of long-term effects. According to Streibelt \& Egner (2012), the time of follow up strongly influences studied outcomes. Accordingly, the benefits of fully qualifying services in terms of more stable employment might only be reflected with a significantly longer follow-up period. Consequently, there is a need for empirical evidence on long-term outcomes to assess the question of long-term effectiveness and efficiency.

In this thesis, the extent to which participation in shorter or longer re-training measures affects a person's ability to return to work is, thus, re-assessed using eight years of follow-up data. The goal is to examine whether program allocation into a one- or two-year program is, on average, more effective in the long run. This research is carried out on two levels: First, average treatment effects are calculated to compare the potential outcomes of all rehabilitants under each treatment scenario over an eight-year post-treatment observation period. Second, the analysis of the treatment effects is limited to the subset of individuals that received a two-year (oneyear) re-training in order to determine the (potential) incremental effects of the longer duration program.

The analysis contributes to a growing body of literature on the comparative effects of publicly funded training programs for people with a disability. Considering a longer post-treatment observation period, this analysis provides new evidence on the relative effectiveness of vocational re-training programs for persons with a disability in Germany.

\section{What are the financial returns for providers of the re-training programs: are the measures cost-effective?}

Whereas legal and social obligations can largely explain expenditures directed at rehabilitation and reintegration, supporting people in the work reintegration process can also be viewed as an economic decision. By enabling workers to stay economically active despite possible health deficiencies, return to work programs can be viewed as important drivers of economic stability and growth (OECD, 2009). Although rehabilitation and reintegration measures are economically important

\footnotetext{
${ }^{2}$ For a more comprehensive survey of the empirical literature, refer to the available reviews: Biewen et al. (2006) and Deeke et al. (2011).
} 
investments, debates about the cost of such measures often dominate public discussions.

To date, systematic cost-benefit analyses that estimate the direct and indirect effects achieved and contrast them with the costs of the measures are generally missing. To assess whether vocational re-training is a worthwhile investment, also from a financial perspective, it is, thus, necessary to itemize the services and their resulting costs to be able to evaluate them economically.

In this thesis, the long-term economic impact of vocational retraining on social security providers' balance sheets is investigated. While considering consumed resources and various economic benefits, cost-benefit relationships of one- and twoyear vocational re-training programs are estimated. The analysis provides new insights into the cost-effectiveness of vocational re-training for people with a disability in Germany, contributing to a better understanding of the economic potential of these measures.

\subsection{Study context and research methods}

\subsubsection{Study context}

Overall there are 3.25 million people in working age in Germany with a severe disability. Contrary to popular belief only $3 \%$ of the disabilities are congenital or occurred in the first year of life. The majority, $88 \%$ of disabilities, are caused by illness developed at some point in life. For this reason, people with a severe disability are disproportionately higher represented in the higher age cohorts. More than 1.3 million persons in Germany, 40 percent of people with severe disabilities in working age, are between 55 and 65 years old. However, the share of this age cohort in all persons of working age is only half as high. (Statistisches Bundesamt, 2017)

The ongoing aging of the German workforce poses challenges to care systems, social security and economic productivity (Weber, Peschkes, \& de Boer, 2015). More than a quarter of the working-age population will be above the age of 55 by 2020 (Weber et al., 2015). Since older workers are more likely to develop a disability, the share of active workers is currently decreasing, a trend that is also related to population aging, which results in a decreasing absolute number of potential workers. In 2017, the German economy was assessed to have a shortage of 440,000 skilled workers (Burstedde, Kolev, \& Matthes, 2017). The effect of this shortage on economic production was estimated to be 30 billion Euros, or equally, a loss of one percent of gross domestic production (Burstedde et al., 2017). In the years to come, the number of workers is predicted to further decrease when the majority of the baby-boomer generation retires (Swiaczny, 2016). 
The sources of disabilities have been changing, too, with an increasing number of disability claims appearing relatively early in working life, exhibiting long claim durations and high survival rates (chronic back problems, mental problems). The average age of persons that have been admitted a pension due to a reduction in earnings capacity is 51.7 years (DRV, 2017). At the same time, legal retirement ages in Germany have been raised to 67 to overcome the ongoing demographic challenges. This extension can already be observed in the statistics of the German Pension Insurance Fund: In 2000, workers admitted into old-age pension were on average 62.3 years old; in 2016 average retirement ages due to old age have increased to 64.1 years (DRV, 2017). These changes mean that, in the case of a disability or illness that prevents the worker from returning to work, the gap between working and retiring is increasing.

The developments described in the previous paragraphs have led to a rapid increase in the economic costs due to longer periods of inability to work, sickness-related unemployment, rehabilitation and early retirement from work. While the total expenditure for the rehabilitation services of the German Pension Insurance Fund increased by approx. 24 percent, from 5.0 to 6.2 billion euros between 1995 and 2010, the share of benefits for vocational rehabilitation increased in the same period from about 648 million to 1,269 billion euros (DRV, 2017). This means that, while about three-quarters of the total costs (2015: about 4.05 billion euros) are attributable to medical rehabilitation, the increase in overall rehabilitation expenditure is, to a large extent, due to the increase in vocational rehabilitation costs. Accordingly, the recent increase in usage of vocational rehabilitation measures demands a critical assessment of the effectiveness of the additional resources spent.

\section{Vocational Rehabilitation in Germany}

This thesis focuses on the employment outcomes of participants in vocational rehabilitation organized under the German Statutory Pension Insurance Fund (Deutsche Rentenversicherung Bund). The people studied throughout this project are individuals who have been provided with a vocational rehabilitation measure as a result of an injuring disability or illness that prohibits them from carrying out former job tasks.

The rehabilitation system of the German Pension Insurance Fund provides good conditions to study the effects of vocational rehabilitation measures on actual labor market outcomes for several reasons. There is a long tradition of implementing different forms of public programs ranging from short measures to medium- and long-term vocational rehabilitation programs. In addition, policymakers have developed a growing awareness of the need to re-evaluate implemented labor 
market policies. This awareness has contributed to researchers gaining access to existing data sources, which can be used for policy analysis.

Vocational rehabilitation is a fundamental element of German social and employment-market policy and is increasingly being used to assist people with disabilities to overcome barriers to employment and facilitate their participation in working life. The observed increase in rehabilitation expenditure described in the previous section results from increased demand for vocational rehabilitation, influenced both by the labor market and demographic changes, and from a greater politician and tax-payer awareness of the importance of vocational rehabilitation for a sustainable return to work. For the taxpayers, it is particularly significant that vocational rehabilitation pays off. The premise "rehabilitation before retirement" is not only a political task; it also reflects the individual and economic gain linked to successful occupational reintegration (Weber et al., 2015).

The German Statutory Pension Insurance Fund is one of Europe's largest providers of vocational rehabilitation measures, investing more than a billion euros every year into vocational rehabilitation (DRV, 2017). Depending on the source of disability and the allocated measures, different implementing agencies are responsible for the provision of vocational rehabilitation services. The type of training measures is usually assigned by a caseworker. Given the regional availability of training programs and other local circumstances, the caseworker may exercise a great deal of discretion in the program allocation process. Suitable programs are chosen by the provider from a large pool of certified private or public institutions specialized in different professions and skill training.

The main goal of vocational rehabilitation is to permanently reintegrate individuals back into employment and to avoid early retirement. The return to work is, thus, the target outcome of vocational rehabilitation and simultaneously an indicator of its effectiveness. The right to vocational guidance and training is manifested in European social law (Council of Europe, 1996, sec. 10) and implemented in Germany through the ninth book of the Code of Social Law (§§49-54, §57, §60 SGB IX $\left.{ }^{3}\right)$

To become eligible for a vocational rehabilitation measure with the German Statutory Pension Insurance Fund, candidates must satisfy any of the following eligibility criteria: Either the applicants 1) already have to receive a pension due to limited earnings capacity, or 2) assessment shows that, without the measure, the pension provider would have to pay out a pension due to limited earnings capacity, or 3) a medical rehabilitation by itself is determined to be insufficient for proper

\footnotetext{
${ }^{3}$ Ninth book of the German Code of Social Law
} 
reintegration into the labor market or, lastly, 4) the qualifying minimum insurance period is fulfilled ${ }^{4}$. Moreover, the training scheme needs to be considered necessary for the rehabilitant and a positive chance for the success of the measures and consequent re-employment should exist. This is the case, for instance, when the chances for employment in the target occupation of the rehabilitant are good but require additional skills or job-specific knowledge. ${ }^{5}$

The allocated measures range from workplace accommodation to professional retraining and education courses being tailored to the individual needs and capabilities of the person with a disability. Overall there are 40 different types of measures, which can be grouped into nine categories: (1) workplace accommodation measures, (2) job preparation measures, (3) vocational re-training measures, (4) workshops for people with disabilities, (5) rehabilitation programs for people with a mental illness, (6) employer services, (7) aptitude testing, (8) mobility grants, (9) startup subsidies. These groups of measures are briefly described below.

(1) Workplace accommodation measures refer to service provisions in which people with disabilities are assisted in obtaining and maintaining employment for example through the provision of technical aids or work equipment. (2) Job preparation measures refer to training courses or counseling sessions aimed at preparing the (disabled) individual for work in the regular labor market. (3) Vocational re-training measures refer to education programs that prepare people to work in professional vocations. (4) Workshops for people with disabilities (sheltered employment) refer to programs consisting of routine-oriented tasks and activities that allow people with disabilities to gain work experience outside the regular labor market before entering the workforce. (5) Rehabilitation programs for people with a mental illness employ specialized psychologists to provide vocational guidance and facilitate labor market reintegration. (6) Employer services refer to direct financial assistance provided to employers for adapting the workplace and for providing training or retraining measures in the company. (7) Aptitude testing refers to tests designed to determine a person's ability in a skill or field of knowledge. (8) Mobility grants refer

\footnotetext{
${ }^{4}$ Depending on the rehabilitation benefit, this may be a waiting period of five or fifteen years, in other cases it is sufficient to have paid compulsory contributions to the pension insurance in at least six calendar months in the past two years prior to the application.

${ }^{5}$ Apart from eligibility criteria, several exclusion criteria also exist: If, due to a work accident or illness, the individual is already entitled to a similar measure with a different rehabilitation provider (e.g. the statutory accident insurance or the unemployment offices); for individuals who already receive an old-age pension of at least two-third of their full pension, or that have applied for one and for civil servants and pension schemes of the liberal professions as well as for retirees and people living mainly abroad.
} 
to assistance provided for acquiring and using a car and for related modifications that accommodate a functional restriction. (9) Startup subsidies refer to grants paid to founders of a new business.

A distinctive feature of the German vocational rehabilitation system are the welldeveloped vocational re-training programs, which provide individuals unable to return to their former employment with an opportunity to learn a new profession. Approximately, one-quarter of all expenditure on vocational rehabilitation is spent on vocational re-training (DRV, 2017). Vocational re-training measures are usually based on manual or practical activities and are traditionally non-academic and related to a specific trade or occupation. Within the pool of available re-training measures, the courses can be classified into partial and full re-training programs. Apart from the fact that both types of re-training programs require full-time participation, they differ considerably in contents and length.

Full-length re-training programs are comparable to regular apprenticeships programs, typically lasting for two years. During the measures, classroom training is combined with on-the-job training to learn skills and obtain a professional qualification in a new field of work. Since their implementation in the 1970s, the measures have received a great deal of acceptance, despite the high importance of professions and professional degrees with regards to finding a job in Germany (Beiler, 2013). Partial qualifications, on the other hand, aim at extending existing competencies with additional skills, e.g. in the fields of business administration or information technology. The measures are usually completed within a year with the aim to reintegrate the participants quickly into working life.

\subsubsection{Data}

In Germany, policymakers have developed a growing awareness of the need to assess and re-evaluate implemented labor market reintegration policies. Establishing a framework to empirically evaluate applied measures is needed to optimize the provision of services and verify that training objectives have been achieved. This need has contributed to researchers gaining access to already existing databases. As a result, informative data sets that merge different administrative sources can now be used for policy analysis. In fact, the collection of program and outcome data is implemented into German social law, a fact that has contributed to researchers gaining access to informative longitudinal program data for program evaluation.

Routinely, the German Statutory Pension Insurance Fund compiles extensive databases of rehabilitation cases that date back to the early 2000s. One of the advantages of the available administrative datasets is that data on income and 
employment of program participants (including graduates of the measures as well as program dropouts) is collected over a long period of time and for representative samples of rehabilitants. Since the data can also be linked to personal socio-economic and health information, the lack of detailed micro-data present in many other evaluation studies can be overcome, enabling micro-founded impact analysis.

The data used for this analysis was collected from the administrative data records of the German Statutory Pension Insurance Fund. The retrieved databases consist of process-generated information on the earnings development and insurance relationship for several cohorts of rehabilitants that entered the vocational rehabilitation program between 2005 and 2009. The so-called "Scientific Use File" SUF_RSDV2013, retrieved in mid-2016, was made available by the "Research Data Centre of the German Statutory Pension Insurance Fund" and consists of four databases.

The first database includes a random sample of all the cases with at least one vocational rehabilitation measure for 2005-2009 and a range of variables linked to implementing the rehabilitation program. Moreover, it provides information on some labor-market-related and personal characteristics at the time of application for treatment. To better account for the health situation, participants' medical discharge information from hospitals and other medical facilities is also included. The data includes information on the type of granted rehabilitation measure, the date of approval, the rehabilitation start date, medical discharge diagnosis (ICD), employment and occupational status at the time of application, residential region and the participants' marital status at the time of application for vocational rehabilitation. The coding name of this first dataset is SUFRSDLV13BFB.

The second dataset was retrieved from the pension insurance follow-up database, which provides information on the participants' insurance relationships. It consists of observations from 2003-2013, including annual individual income and days of (un-)employment data. It, thus, overcomes the lack of detailed labor market data present in many other studies. In this study, the following variables are used from this file: year, yearly income, annual days of employment, days with short- or longterm unemployment benefits and days with other social-security benefits. The coding name of this second dataset provided is SUFRSDLV13BYB

To provide further information on the participants' socio-demographics, a third dataset was merged with the previous files. The latter file includes data on the persons' sex, birth (death) years, nationalities and highest attained levels of education. The coding name of the third dataset provided is SUFRSDLV13KOB 
A fourth dataset provided information on receiving a pension. The data comprises information on whether a pension due to a reduced earnings capacity was paid out as well as on the level of the pension benefits awarded. The coding name of the fourth dataset provided is SUFRSDV13RTB

To retrieve information on the employment status before and after participation in vocational rehabilitation, the longitudinal labor market data was merged with the rehabilitation data. The unique identifier, present in all four databases used to merge the data, is the variable "case". Data management and analysis were performed using the statistical software Stata $14.2^{6}$.

\subsubsection{Econometric approach}

The main part of this thesis consists of four empirical chapters that are each based on the previously introduced administrative data set, retrieved from the German Statutory Pension Insurance Fund. The underlying framework for the analysis of program effects is that of human capital theory: Human capital theory assumes that education develops skills, that these skills increase a worker's capacity to be productive and that increased productivity leads to better employment outcomes.

The starting point of the quantitative analysis was the measurement of employment outcomes for several years before and after participation in a vocational rehabilitation measure associated with the German Statutory Pension Insurance Fund. The analysis then proceeded to estimate 1) the partial effects of socioeconomic determinants on these employment outcomes, 2) the treatment effects associated with vocational re-training, 3) the incremental effects of the more involved re-training program, and 4) the effects of vocational re-training on the social security system.

The following paragraphs summarize the econometric approach taken to answer the research questions formulated in the previous section.

1) The research design in the analysis of socio-economic factors can be described as a before-after comparison as differences in pre-post outcomes among different groups of rehabilitants were assessed. Ordinary least squares regression models were used to derive partial regression coefficients for individual-level determinants. First, a multivariate analysis of the variance of before-after differences in employment outcomes was performed (gain score approach). Second, a lagged dependent variable was included, and the

\footnotetext{
${ }^{6}$ All estimations were carried out using the statistical Software Stata 14.2. StataCorp LLC, 4905 Lakeway Drive, College Station, Texas 77845-4512, USA.
} 
calculation was repeated considering heterogeneity in pre-treatment employment outcomes among socio-economic groups. Both steps aimed to provide an estimate on the size and direction of partial regression coefficients for individual-level rehabilitant characteristics. In the analysis, rehabilitants were differentiated by demographic as well as by work-related characteristics such as age, gender, job position or last employment status, thus allowing an evaluation of the partial effects of these factors on before-after changes in income, employment days and days with short-term unemployment benefits.

The two types of regression setups were included in this analysis because they provide answers to different questions. The first, gain score analysis, yields the more intuitive outcomes illustrating the overall income and employment development of different socio-economic groups of rehabilitants. However, those with larger pre-test outcomes can generally experience larger changes than those with lower scores at the pre-test when using a change-score approach. This is because without any employment in the years before entering the rehabilitation program, the change score cannot be negative. Thus, an alternative estimate is provided, illustrating the income and employment development of the different socio-economic groups, given that the rehabilitants would have earned the same before program admission. Since, in comparison to a change score analysis, in the second, lagged dependent variable, approach the pre-test outcomes enter the regression function as covariates (instead of being subtracted from the post-rehabilitation outcomes), the corresponding error distribution can be used to explain the variation (variance) in posttreatment outcomes. This procedure allows a different interpretation compared to the first analysis.

2) The employment effects associated with vocational re-training were analyzed through retro-perspective quasi-experiments. In the analysis, a "selection on observed variables strategy" was adopted making use of available pre-treatment data on the rehabilitants' socio-economic statuses to identify conditional treatment-probabilities. The set of independent variables included variables for the treatment status, representing the type of vocational rehabilitation program a rehabilitant participated in, as well as of micro-level covariates consisting of individual and environmental contextual factors.

The program impact was calculated using inverse probability weighted regression adjustment. Specifically, the average treatment effect and the average treatment effect on the treated of completion of re-training were estimated against the alternatives of non-completion. In the absence of a natural 
comparison group, an internal comparison group design was used to compare outcomes with and without vocational re-training. In a much-cited academic paper, Dean \& Dolan (1991) argue that the preferred control group in an evaluation of training programs should consist of clients who enroll in the services and get admitted but never graduate from the program. This is because using program dropouts reduces concerns about selection bias since dropouts and completers share the same motivation to apply for the vocational re-training program, satisfy the relevant eligibility criteria and probably exhibit similar levels of (unobserved) severities in their disabilities. To examine the effect of vocational re-training on labor market reintegration, several outcome measures were used: The primary estimated outcome variable was the accumulative income earned in the first eight years after program admission. Secondary outcomes consisted of days with employment and the number of days with unemployment and other social-security benefits. ${ }^{7}$

3) Often valid control groups can also be found by analyzing participants in competing vocational education programs. These groups are permissible in this research because the best alternative to a certain educational program is not necessarily to receive no education; it might be to participate in a different program instead. To test for the differential effects of one- and two-year retraining programs, treatment effects of both programs were directly compared. Specifically, the average treatment effect on the treated of participation in twoyear re-training programs was estimated against the alternatives of participation in shorter, partial re-training to examine whether the additional year of education "paid-off" from the perspective of the participant.

4) To evaluate the cost-effectiveness and cost-efficiency from the perspective of social security, the benefits of vocational rehabilitation to providers were compared with the incurred expenditure. The main challenge in the analysis of economic effects was to quantify the different benefits and costs of the interventions and to develop adequate predictions about the future development of received wages and benefits. In this study, the benefits were quantified based on the results of the quasi-experiments described in chapters three and four while costs were based on a combination of primary data and information retrieved from secondary literature.

First, the direct effects on wages and benefit receipt were extrapolated to provide out-of-sample estimates on the impact of vocational re-training measures over

\footnotetext{
${ }^{7}$ After 2010, information on long-term (means-tested) unemployment benefits is unavailable due to changes in legislation.
} 
an entire working life. After extrapolating the treatment effects, additional tax collection and contribution collection as a result of increased income were calculated. Furthermore, prevented un-employment and other social-security benefit pay were monetarized and factored in. This process provided the basis for calculating benefits for social security providers. The cost-effectiveness and efficiency of the measures were, then, evaluated using a break-even analysis, net present value analysis, return-on-investment analysis and by calculating the internal rate of return.

\subsection{Outline of the thesis}

The book comprises six chapters. In Chapter 1, the aims and contributions of the thesis and the research questions have been introduced. Chapter 2 provides a detailed overview of the influence of socio-economic factors on changes in employment outcomes before and after participation in a vocational rehabilitation measure. Chapter 3 investigates the impact of vocational re-training before the duration effects of the re-training programs are further assessed in Chapter 4. Chapter 5 introduces the costs of re-training and secondary benefits to social security into the calculation to assess whether the applied measures are cost-effective from the perspective of (public) social security systems. Chapter 6 presents the conclusions of this thesis. Below is a brief description of each chapter.

\section{Chapter 2: The role of socio-economic factors}

In the second chapter, the extent to which differences in employment outcomes can be linked to the participants' socio-economic backgrounds is estimated. This chapter comprises a longitudinal analysis of the income and employment outcomes of multiple cohorts of rehabilitants. The focus of this chapters' analysis is on differences in these employment outcomes, linked to the participants' socio-economic characteristics. Since participants in vocational rehabilitation are a heterogeneous group of people, with different social and occupational backgrounds and different health situations, possible barriers to work reintegration can only adequately be assessed with reference to the rehabilitants' individual situations. A needed step to improve the empirical evidence is, thus, to assess the relevant person-related factors influencing return-to-work outcomes. This chapter provides an overview of the direction and size of the relevant person- and occupation-related factors such as age, gender, education, employment history, job type as well as other relevant socioeconomic characteristics.

\section{Chapter 3: The impact of vocational re-training on employment outcomes}

In the third chapter, the impact of vocational education is estimated by drawing a comparison between program completers and non-completers. An impressive 
amount of literature has shown that individual wages increase with general education; whether this holds also for vocational education measures for people with a disability is unclear: So far there is only limited evidence on the effectiveness of vocational education for people with a disability. This chapter evaluates the size of the absolute treatment effects of one- and two-year programs on work participation outcomes in Germany.

\section{Chapter 4: One-and two-year re-training in comparison: does the extra year pay off?}

In the fourth chapter, the extent to which one-year and two-year vocational retraining programs have similar effects on employment outcomes is further examined. Although the shorter re-training programs require less time and smaller financial resources, recent assessments have found that they might provide employment effects comparable to the traditional two-year programs. In this analysis, this claim is challenged based on longitudinal cohort data of participants of vocational rehabilitation. Through the calculations, it is possible to determine which type of programs have a larger impact on labor market outcomes for the average participant. Thereby, this chapter's analysis contributes to closing a relevant gap in prior literature on the effects of competing treatment alternatives/strategies.

Chapter 5: The return on vocational re-training: cost-effectiveness from the perspective of social security

In the fifth chapter, the long-term impact on the social security systems' financial balance sheet is evaluated. While considering consumed resources and economic factors such as inflation, cost-benefit calculations of one- and two-year re-training programs are performed. By providing new evidence on metrics such as the net present value, the return on investment or the internal rate of return, this chapter's analysis contributes to a better understanding of the economic consequences of vocational re-training.

\section{Chapter 6: Conclusions and policy implications}

The sixth chapter presents the conclusions of this thesis. It comprises a summary and discussion of the gathered evidence with regards to answering the four research questions posed in the introduction as well as a discussion on policy implications, possible limitations of this study and recommendations for future research. The thesis concludes with a summary of the main scientific contributions. 
2. The role of socio-economic factors 


\subsection{Introduction}

One important step in improving the provision of vocational rehabilitation measures is providing an assessment of the relevant person-related and environmental factors influencing long-term employment outcomes. Because people with a disability and those at risk of becoming disabled do not constitute a homogeneous group of people, possible barriers to work reintegration can only be adequately assessed with reference to the rehabilitants' individual situations (Hoffmann, 2004).

Many studies on labor market consequences of vocational rehabilitation do not present disaggregated results by age groups, gender and other environmental variables. Yet there is a wide demand for representative empirical data as detailed results are needed to be able to investigate and react to the specific needs and responses of individuals with regards to vocational rehabilitation.

In research on the return to work of people with a disability, the complex relationship between demographic and work factors has been evident for some time (Baril, Berthelette, \& Massicotte, 2003; Foreman, Murphy, \& Swerissen, 2006; Muijzer, Groothoff, De Boer, Geertzen, \& Brouwer, 2010)). In particular, the factors age, gender and health status have repeatedly been shown to significantly affect rehabilitation outcomes (Gericke, 2010; Meschnig, von Kardorff, \& Klaus, 2019; Schmidt, Flach, \& Begerow, 2011; Streibelt \& Egner, 2012b).

Age and return-to-work outcomes are negatively correlated (Blackwell et al., 2004; Cancelliere et al., 2016; Dodoo-Schittko et al., 2017; Valentin et al., 2016; Van Muijen et al., 2013). A possible explanation is that older workers are less likely to adapt to new situations and have poorer chances on the labor market than younger applicants. Moreover, many elderly workers have less of an incentive to return to work as they are often only a few years away from retirement.

With regards to the effects of gender, empirical results are similarily consistent, demonstrating that males generally have higher return-to-work outcomes compared to females, everything else being equal (Cancelliere et al., 2016; Cornelius et al., 2011; Feuerstein et al., 2001; Giesen J. \& Cavenaugh Brenda, 2013; Saltychev et al., 2013; Stergiou-Kita et al., 2016). A frequently made argument here is that the lower performance of females with regards to labor market reintegration might be the result of a male-centered work environment, the invisible "glass ceiling" or greater physical stress and time demands outside the working place being placed on women.

The majority of findings on the effect of the marital status of the rehabilitants on work outcomes indicate that married individuals are more likely to return to work 
and that the divorced are particularly vulnerable to staying out of work (Kreutzer et al., 2003; Selander et al., 2002; Street \& Lacey, 2015; Yasuda et al., 2002). While not all authors agree on the causes, the positive effect of being married on rehabilitation outcomes is thought to be partially attributed to the social support received through marriage. Singles are at a greater danger of leaving the labor market permanently.

The level of prior education has also been found to be strongly correlated with return-to-work outcomes (Blackwell et al., 2004; Cancelliere et al., 2016; Giesen J. \& Cavenaugh Brenda, 2013); the likelihood of successfully returning to work increases with additional education. The research results suggest that the improved outcomes for more highly educated individuals may be the result of greater adaptability with regards to coping with the health effects of disabilities, as well as with regards to possible changes in occupational activities. Other, more difficult to measure, characteristics affecting employment results are, for example, the individual motivation for work, intelligence, determination, commitment or the ability to cope with stress (Gericke, 2010).

Concerning the rehabilitants' work and professional lives, several studies found that individual work history significantly affected employment outcomes. The history included past income and employment developments, as well as employment or job type related indicators (see for example Adams \& Williams, 2003; Cornelius et al., 2011; Giesen J. \& Cavenaugh Brenda, 2013; Ottomanelli \& Lind, 2009). Blue-collar and manual labor is usually associated with reduced employment outcomes. Moreover, a supportive work environment and the status of employer-employee relations have been found to significantly affect return-to-work rates (Franche et al., 2005; A. Muijzer et al., 2011).

This chapter contributes from a labor market perspective to the literature on the topic of vocational rehabilitation. It does so by answering open questions with regards to measuring long-term employment outcomes and by estimating the socioeconomic determinants on a rehabilitant level. For 19,250 participants of vocational rehabilitation in the German labor market, results four years before and after program entry were retrieved and compared. This comparison examined the effects of socio-economic status by determining to what extent differences in attained employment outcomes could be linked to the rehabilitants' demographic and occupational statuses.

This chapter is organized as follows: The methods section presents the study design and population, the data and the econometric approach. In the results section, the development of employment outcomes over time is analyzed before estimates of the 
influence of the measured socio-economic factors on changes in the employment status are presented.

\subsection{Methods}

\subsubsection{Study design and population}

The research design for the analysis of socio-economic determinants can be categorized as a before-after comparison. In the analysis, before-after differences in employment outcomes among socio-economic groups of rehabilitants were estimated. The starting point for the empirical analysis was the collection of employment data on people who had participated in a vocational rehabilitation measure with the Germany Statutory Pension Insurance Fund in the years 20072009. Subsequently, an analysis of variance and covariance of these employment outcomes was performed to estimate partial regression coefficients for individuallevel rehabilitant characteristics registered. Rehabilitants were differentiated by demographic and work-related characteristics including their age, gender, marital status and nationality, place of residence, job position, employment status and prior employment history. Furthermore, to control for possible health differences among participants, medical discharge information from hospitals and other medical facilities was included as a control.

\subsubsection{Data}

The retrieved data consisted of a random sample of all vocational rehabilitation measures approved by the insurer between 2005 and 2013. The "Scientific Use File", retrieved in mid-2016, was made available by the "Research Data Centre of the German Pension Insurance" and was composed of three databases: The first database included a random sample of all the cases with at least one vocational rehabilitation measure and a range of variables linked to the implementation of the rehabilitation program. Moreover, it provided information on some labor-market-related and personal characteristics at the time of application for treatment.

In a second step, the rehabilitation data was merged with longitudinal labor market data to retrieve information on the employment status before and after the participation in vocational rehabilitation. That employment data was retrieved from the pension insurance follow-up database, which provides information on the insurance relationship of the participants. It consisted of observations from 20032013, including data on yearly individual income and days of (un-)employment, overcoming the lack of detailed labor market data present in many other studies. To provide further information on the participants' socio-demographics, a third dataset was merged with the previous files. The latter file included data on a person's gender, birth year, nationality and highest attained level of education. 
The first challenge was to identify the year in which a rehabilitant entered a program. To identify the correct year for matching in the rehabilitation file, the rehabilitation start date was used. When no start data was entered, the case when, for example, measures consisted only of the provision of technical aids, the date of approval was used. In a second step, the income and employment observations from several years before and after participation were used to generate timely lagging variables added horizontally to each rehabilitation case. The resulting crosssectional dataset provided information on the rehabilitants' income and employment developments over several years before and after participation in vocational rehabilitation.

While most participants received more than one vocational rehabilitation measure over the observation period, the focus of this chapter's' analysis was on comparing income and employment results four years before and after entry into the vocational rehabilitation program. Data was, thus, limited to people who were allocated a vocational rehabilitation measure under the Germany Statutory Pension Insurance Fund from 2007-2009. The included cases were further limited to program entries for which labor market outcomes were available for all four years before and after participation in vocational rehabilitation and where all the participants' baseline characteristics (socio-economic variables) were recorded. Moreover, to limit the observations to those in working age, rehabilitants below 20 and above 62 years of age were dropped from the dataset. The final sample consisted of 19,250 cases for which complete employment data was available, four years before and after participation in vocational rehabilitation, and for which all socio-economic variables were recorded.

\section{Outcome variables}

The rehabilitation literature has proposed several approaches with regards to the measurement of employment outcomes of interventions (see for example Steenstra, Lee, De Vroome, Busse, \& Hogg-Johnson (2012) for a recent review); most commonly return-to-work is measured in some way as it is the primary goal of vocational rehabilitation programs. Return-to-work outcomes refer to an observation of the working status, at a period or point in time after experiencing a disabling disease or accident and often imply a comparison with the situation before.

In this chapter, the following indicators were used as outcomes:

(1) Nominal income, (2) real income, (3) days with employment, (4) days with short term unemployment benefits 
Nominal income refers to income from labor that was subject to social insurance contributions. Income data collected by the German pension insurance is limited to a contribution ceiling. For annual incomes above $€ 69,600$ ( $€ 58,800$ in the former East German states), the median value of $€ 77,179$ (€65,400) was recorded in the data instead of the true value. This potentially lowers the estimates, as annual income above the specified cut-off value was not properly recorded. Changes in real (inflation-adjusted) income were estimated using the average of historical inflation rates collected from the German Federal Bureau of Statistics between 2003 and 2013 as the discount factor (Destatis, 2018). The mean annual discount rate used was equal to $1.6 \%$. Using this value, all income flows in the four years before rehabilitation start, and in the four years after, were discounted into the year of rehabilitation admission (i.e. 2007-2009). Days with employment included all employment days including days with sickness absence benefits, if the employer is still obliged to pay wages. Short-term unemployment benefits are benefits of the German unemployment insurance, which are paid on the occurrence of unemployment. They are usually paid for up to one year after having lost employment, and for older unemployed people, for up to two years.

\section{Explanatory variables}

Among the group of explanatory variables, a focus was placed on the age-specific differences, as elder rehabilitants are generally considered to be particularly vulnerable with regards to labor market reintegration after having experienced a disabling injury or illness. To test, whether the outcomes of elderly workers differed to those of younger workers, age groups were created and included as indicator variables. Moreover, to detect differences in labor market outcomes linked to the rehabilitants' genders, an indicator variable for being female was included. Moreover, the effect of marital status and nationality were considered; a set of indicators were included for each category.

Prior educational attainments were considered through the inclusion of variables reflecting the level of schooling and professional education at program entry. Furthermore, employment and job-related dummy variables were included, such as the employment status before rehabilitation and the last job type. Further covariates were the treatment type, the residential region, the medical discharge diagnosis (ICD-10) and the calendar year of rehabilitation start. While the focus of the analysis was the socio-economic factors, it was, nevertheless, important to control for differences in treatment since the various programs have different lengths and could, therefore, also have a different impact on subsequent employment outcomes. 


\subsubsection{Econometric approach}

Two types of regression models were used to estimate partial regression coefficients for individual-level factors. The first approach was a multivariate analysis of the variance of differences in employment market outcomes before and after participation in a rehabilitation measure (gain score approach). The second method used a lagged dependent variable to better account for rehabilitant heterogeneity in pre-treatment outcomes. Both estimates aimed to provide an estimate on the size and direction of partial regression coefficients for individual-level rehabilitant characteristics.

The reason for including these two types of regression setups in this analysis is that they provide answers to different questions. Analysis using gain scores yields the more intuitive outcomes, illustrating the overall income and employment development of different socio-economic groups of rehabilitants. However, in our setting, those with better pre-test employment outcomes can generally experience greater changes than those with lower scores at pre-test (they have more to lose) rehabilitants without any income in the four years before entering the program cannot experience a negative income development, thus, the change score can only be positive. Therefore, an alternative estimate is provided, illustrating the income and employment development of the different socio-economic groups, given that the rehabilitants would have earned the same at the start of the program.

In comparison to a change score analysis, in a lagged dependent variable approach, the pre-test scores enter the regression function as covariates. The corresponding error distribution can, thus, be used to explain the variation (variance) in posttreatment outcomes. This method allows a different interpretation compared to the first analysis. The technical discussion between gain score and covariate adjustment is well documented in the scientific literature (see e.g. Glymour, Weuve, Berkman, Kawachi, \& Robins, (2005) or Maris (1998) for a more extensive discussion).

\section{Change score regression approach}

The change score, defined as the difference between pre- and post-test results, is frequently used as the dependent variable in the comparison of two or more groups. Using the gain score approach, it is possible to control for differences in pre-test scores at an individual level by measuring the post-test score relative to every individual's pre-test score. However, it is not possible to control for differences in pre-test scores between groups. As a result, using change scores when measuring changes has been criticized, commonly with the argument that the scores are less reliable than the raw values. The reliability depends on the nature of the data, for instance, whether the pre-test outcomes have a causal effect on the post-test results or whether the included explanatory variables are correlated with the pre-tests 
(Allison, 1990). To compare the employment outcomes after participation in vocational rehabilitation with the situation before (using the change score regression approach), the dependent variable was constructed as follows:

$y_{\Delta}=\left(\boldsymbol{y}_{t+1}+\boldsymbol{y}_{t+2}+\boldsymbol{y}_{t+3}+\boldsymbol{y}_{t+4}\right)-\left(\boldsymbol{y}_{t-1}+\boldsymbol{y}_{t-2}+\boldsymbol{y}_{t-3}+\boldsymbol{y}_{t-4}\right)$

\section{Lagged dependent variable approach}

In settings where starting positions are heterogeneous, using gain scores might be misleading while using pre-test scores as a covariate can, to a large extent, reduce error variance and eliminate systematic bias. This improvement results from including lagged values of the dependent variable into the group of controls, which allows the differences in pre-test scores between groups to be considered. The resulting estimates provide information on partial effects, given equalized pre-test scores of the dependent variable. The interpretation of the regression coefficients, thus, differs compared to that in the change score set-up, in which the estimates for the size of the coefficients are retrieved irrespective of pre-test results. Accordingly, additional OLS regressions with before rehabilitation outcomes as part of the group of explanatory variables were estimated. The pre-treatment outcomes of the dependent variables were no longer part of the dependent variable. Instead, the dependent variable equaled the sum of the employment outcomes in the four years after rehabilitation while the before-rehabilitation outcomes entered the specification only as covariates:

$y_{\text {level }}=\left(\boldsymbol{y}_{t+1}+\boldsymbol{y}_{t+2}+\boldsymbol{y}_{t+3}+\boldsymbol{y}_{t+4}\right)$

\subsection{Results}

\subsubsection{Characteristics of the study population}

Table 1 presents the descriptive statistics of the rehabilitants. The average age of the 19,250 people in the sample at the start of the measures was 45.8 years. Furthermore, age groups were defined with an interval of five years. The age groups indicated that most participants were between $36-55$, whereas only $17.1 \%$ were 35 or younger and only $13.8 \%$ of the participants were aged above 55 .

With regards to gender, $46 \%$ of the rehabilitants were male while $54 \%$ were female. $24 \%$ were single, $60 \%$ were married, $14.4 \%$ divorced and $1.6 \%$ widowed at the start of the rehabilitation measures. $97.9 \%$ had German nationality, with the Turkish nationality being the largest minority with $0.5 \%$. Moreover, most of the participants were not highly qualified (only $4.9 \%$ had a university degree, $10.5 \%$ with a technical college degree). On the contrary, most of the participants had a relatively low educational attainment. $70.5 \%$ did not have a full high school education (in Germany 
called "Abitur"). However, many participants did hold supplementary apprenticeship degrees, which are common in Germany.

Table 1: Descriptive Statistics, demographic variables

\begin{tabular}{lr}
\hline Variable & Distribution \\
\hline Age (in \%, mean = 45.8 years) & \\
$20-25$ & 9.0 \\
$26-30$ & 2.5 \\
$31-35$ & 5.6 \\
$36-40$ & 14.7 \\
$41-45$ & 23.0 \\
$46-50$ & 23.7 \\
$51-55$ & 19.4 \\
$56-60$ & 9.8 \\
61-62 & 4.0 \\
Sex (in \%) & \\
Male & 46.0 \\
Female & 54.0 \\
Marital Status (in \%) & \\
Single & 24.0 \\
Married & 60.0 \\
Divorced & 14.4 \\
Widowed & 1.6 \\
Nationality (in \%) & \\
German & 97.9 \\
South Europe & 0.4 \\
Former Yugoslavia & 0.4 \\
Turkey & 70.5 \\
Other & 4.9 \\
Education (in \%) & 0.5 \\
No high school no apprenticeship & \\
No high school with an apprenticeship & \\
High school no apprenticeship & \\
High school with an apprenticeship & 0.8 \\
Technical College & \\
University degree & \\
& \\
&
\end{tabular}

Table 2 shows whether the individuals were employed before the start of the measures. The table indicates that most rehabilitants were employed, either full- or part-time, white-collar workers. Only $13.8 \%$ were registered as unemployed. 


\begin{tabular}{lr}
\hline Variable & Distribution \\
\hline Employment status (in \%) & \\
Full-time work & 59.0 \\
Full-time piece work & 6.9 \\
Fulltime with nightshifts & 2.8 \\
Part-time work < 50\% & 1.7 \\
Part-time work > 50\% & 15.4 \\
Other & 0.5 \\
Unemployed & 13.8 \\
Last Job Type (in \%) & \\
Unskilled blue-collar & 5.0 \\
Low skilled blue-collar & 3.9 \\
Skilled blue-collar & 17.7 \\
Master / Craftsmen & 1.0 \\
White-collar & 72.0 \\
Self-employed / civil servant & 0.4
\end{tabular}

Table 3 presents the distribution of vocational rehabilitation measures together with the main medical diagnosis that led to admission into rehabilitation. Most rehabilitants received some form of assistance for keeping or obtaining a job (59.6\%); second most common were vocational education measures $(14 \%)$, followed by aptitude testing (10\%). The other groups of measures were sheltered employment $(5 \%)$, mobility grants $(4.3 \%)$, vocational preparation courses $(3.8 \%)$, employer services $(2.5 \%)$ as well as specific measures for the mentally ill $(0.7 \%)$ and start-up subsidies $(0.2 \%)$.

Overall, 166 distinct medical identifiers were used in the analysis to reflect the rehabilitants' health situations at the start of the vocational rehabilitation measure. With regards to the main registered medical diagnosis, Dorsalgia $(28.06 \%)$, a disorder characterized by marked discomfort sensation in the back region was the most frequently registered medical problem. The second most frequently registered medical problems were thoracic, thoracolumbar, or lumbosacral intervertebral disc disorders $(15.39 \%)$ followed by other and unspecified disorders of the back or spine $(3.92 \%)$, conductive and sensorineural hearing loss $(3.10 \%)$ and cervical disc disorders (2.21\%). 
Table 3: Descriptive Statistics, distribution of assigned vocational rehabilitation measures and main medical diagnosis

\begin{tabular}{lr}
\hline Variable & Distribution \\
\hline Type of VR measure (in \%) & \\
Work accommodations & 59.6 \\
Vocational education & 14.0 \\
Aptitude testing & 10.0 \\
Sheltered employment & 5.0 \\
Mobility grant & 4.3 \\
Vocational preparation & 3.8 \\
Employer services & 2.5 \\
Rehab for mentally ill & 0.7 \\
Startup subsidy & 0.2 \\
Main medical diagnosis (in \%) & \\
Dorsalgia (M54) & 28.06 \\
Thoracic, thoracolumbar, and lumbosacral intervertebral disc & 15.39 \\
disorders (M51) & 3.92 \\
Other and unspecified dorsopathies (M53) & 3.10 \\
Conductive and sensorineural hearing loss (H90) & 2.21 \\
Cervical disc disorders (M50) & 1.72 \\
Other and unspecified hearing loss (H91-95) & 1.71 \\
Osteoarthritis of the hip (M16) & 1.70 \\
Depression (F32)
\end{tabular}

\subsubsection{Changes in employment outcomes}

The mean income results for four years before and after participation in vocational rehabilitation are summarized in Table 4. The development of nominal labor income illustrates the loss in earnings capacity before program entry: In the four years before program admission, mean nominal income was equal to, respectively, $€ 26,066$, $€ 26,237, € 25,969$ and $€ 23,701$, In the year when the rehabilitants were admitted into vocational rehabilitation, the mean income was equal to $€ 21,998$. After program admission, mean income increased to $€ 24,209$ in the first year, $€ 25,030$ in the second year, $€ 25,838$ in the third year and $€ 26,099$ in the fourth year after program admission.

In the last row, the accumulative income in the four years after admission is also compared to the accumulative results in the four years before admission. The average change score in nominal income was equal to $€-799$, while in real terms the before-after difference equaled $€-8,975$. The attained statistics exhibit large standards deviations, indicating a lot of variation (volatility) in program outcomes, which is an 
important prerequisite for using the change score as the dependent variable in subsequent analysis. Summarizing, income was generally gradually reduced in the years before rehabilitation and then consistently increased in the following years.

With regards to the number of days worked, a similar development over time can be observed. While, four years before program participation, the mean number of days employed was equal to 302, it was reduced to 256 in the year before the start of the rehabilitation program. In the year of program entry, rehabilitants only worked an average of 226 years. In the four years after program admission, the average number of days employed increased to 249, 256, 263 and 261 days, respectively. The pre-post change score equaled -119.65 days in employment. Summarizing, the rehabilitants had fewer and fewer days in employment over the four years before rehabilitation measures. After the measure, the number of days in employment then gradually rose over the following four years to a level under that of four years before the measure.

With regards to average days with short term unemployment benefits, values rose from 17 days four years before program admission to almost 20 days in the year before rehabilitation start and 26 days in the year entering the vocational rehabilitation program. After program entry, values decreased to 17 in the first year, to 15 days in the second and third year after admission, down to 13 days in the fourth year after admission into rehabilitation. The mean change score was equal to -7.61 days. Summarizing, in the four years after rehabilitation measures, the rehabilitants received benefits on increasingly fewer days than in the four years before the measures.

While days of employment and days with unemployment correlate with each other, there are also some discrepancies because not every day without employment automatically yields unemployment benefits. First, not all individuals apply for unemployment benefits, for which they are entitled due, e.g. because periods of unemployment may be too short. Second, some people might decide to spend more time outside the traditional labor market (voluntary work, household work, etc.) or change their work-leisure balance. 


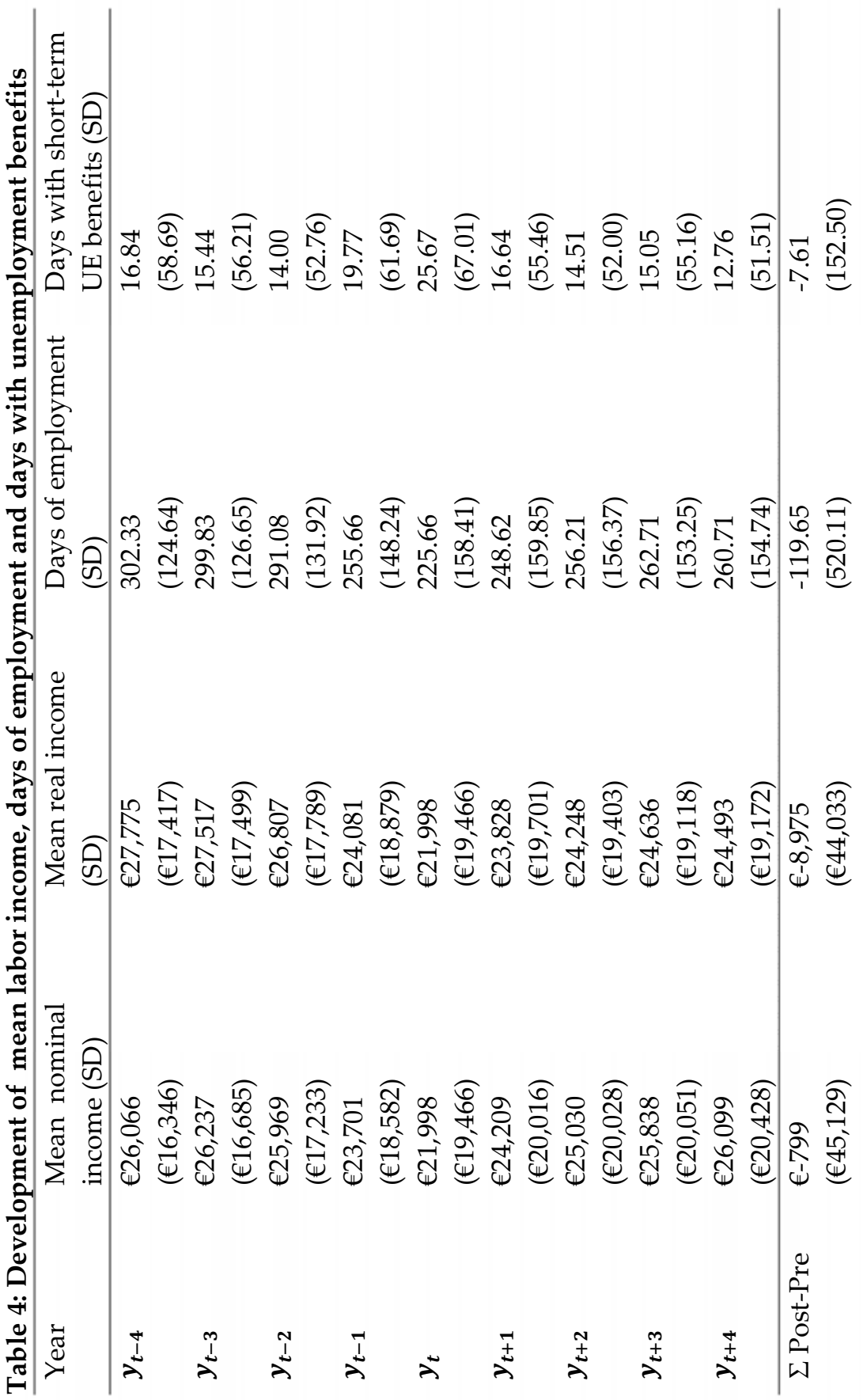




\subsubsection{Regression results Change score regression results}

Table 5 presents the regression results for the before-after rehabilitation differences in income, days with employment and days with unemployment benefits of the gain score regressions. In total, respectively, 31\%, 29\%, 32\% and 10\% of the variation in pre-post-employment outcomes was explained through the factors included in the regression. Regarding the demographic predictors, age has been found to have a significant effect on the change score. Everything else equal, the pre-post difference in income was much lower with higher ages. The lower income changes were a result of fewer days in employment changes with greater ages. On the other hand, a greater age was also associated with larger pre-post differences in the number of days on short-term unemployment benefits.

For the rehabilitants' genders, the regressions showed that females were associated with a lower-wage loss than men over the four years, respective to their own starting position. Moreover, females had a smaller reduction in days of employment, about six and a half days per year. The size of the estimated coefficients was relatively small, yet significant at the $1 \%$ level. Differences in the number of days with short term unemployment benefits seemed not to be related to differences in gender.

To evaluate the influence of the family situation, the marital status of the rehabilitants was also examined. The coefficients for married and divorced were statistically significant; however, their absolute sizes were small in comparison. The next factor assessed was the educational background. The results indicate that the before-after difference changed significantly more with higher education; compared to rehabilitants without a high school diploma (abitur), those with a high school, college or university degree had significantly higher changes in pre-post earnings, everything else being equal.

With regards to the job type, white-collar workers and the self-employed had significantly higher changes in income and in the number of days with employment than blue-collar workers. Piece work and nightshift had a negative effect on earnings differentials compared to regular full-time work, while previously working in parttime had a positive effect on the change score. Moreover, the estimates for the employment status show that unemployed rehabilitants had the highest pre-post changes of all groups compared. 
Table 5: OLS regression of socio-economic factors on the before-after difference in income, employment days and short-term unemployment benefits

\begin{tabular}{|c|c|c|c|c|}
\hline Variable & $\begin{array}{c}\text { Nominal } \\
\text { Income }(€)\end{array}$ & $\begin{array}{c}\text { Real } \\
\text { Income }(€)\end{array}$ & $\begin{array}{c}\text { Employed } \\
\text { (in days) }\end{array}$ & $\begin{array}{l}\text { Short-term } \\
\text { UE (in days) }\end{array}$ \\
\hline \multicolumn{5}{|l|}{ Age (base $=20-25)$} \\
\hline $26-30$ & $\begin{array}{c}-9275.67^{* *} \\
(3140.37)\end{array}$ & $\begin{array}{l}-9645.89 * * \\
(3101.55)\end{array}$ & $\begin{array}{l}-16.38 \\
(41.53)\end{array}$ & $\begin{array}{c}8.20 \\
(13.19)\end{array}$ \\
\hline $31-35$ & $\begin{array}{c}-18643.94^{* * *} \\
(2973.15)\end{array}$ & $\begin{array}{c}-19866.15^{* * *} \\
(2938.47)\end{array}$ & $\begin{array}{l}-63.64 \\
(38.32)\end{array}$ & $\begin{array}{l}33.18^{* *} \\
(12.10)\end{array}$ \\
\hline $36-40$ & $\begin{array}{c}-17651.48^{* * *} \\
(2799.05)\end{array}$ & $\begin{array}{c}-19353.16^{* * *} \\
(2765.87)\end{array}$ & $\begin{array}{l}-47.55 \\
(36.55)\end{array}$ & $\begin{array}{l}31.07^{* *} \\
(11.56)\end{array}$ \\
\hline $41-45$ & $\begin{array}{c}-19039.78^{* * *} \\
(2783.53)\end{array}$ & $\begin{array}{c}-21197.74^{* * *} \\
(2749.37)\end{array}$ & $\begin{array}{l}-41.06 \\
(36.35)\end{array}$ & $\begin{array}{l}34.55^{* *} \\
(11.47)\end{array}$ \\
\hline $46-50$ & $\begin{array}{c}-24025.10^{* * *} \\
(2800.11)\end{array}$ & $\begin{array}{c}-26276.92^{* * *} \\
(2765.63)\end{array}$ & $\begin{array}{l}-73.24^{*} \\
(36.52)\end{array}$ & $\begin{array}{l}37.93^{* *} \\
(11.53)\end{array}$ \\
\hline $51-55$ & $\begin{array}{c}-29344.38^{* * *} \\
(2831.75)\end{array}$ & $\begin{array}{c}-31543.76^{* * *} \\
(2796.79)\end{array}$ & $\begin{array}{c}-110.65^{* *} \\
(36.75)\end{array}$ & $\begin{array}{c}41.19^{* * *} \\
(11.69)\end{array}$ \\
\hline $56-60$ & $\begin{array}{c}-35816.25^{* * *} \\
(2935.36)\end{array}$ & $\begin{array}{c}-37776.02^{* * *} \\
(2897.03)\end{array}$ & $\begin{array}{c}-147.78^{* * *} \\
(37.57)\end{array}$ & $\begin{array}{c}64.70^{* * *} \\
(12.23)\end{array}$ \\
\hline $61-62$ & $\begin{array}{c}-39096.50^{* * *} \\
(5013.30)\end{array}$ & $\begin{array}{c}-40853.53^{* * *} \\
(4941.74)\end{array}$ & $\begin{array}{c}-211.35^{* * *} \\
(54.15)\end{array}$ & $\begin{array}{c}81.48^{* * *} \\
(21.50)\end{array}$ \\
\hline \multicolumn{5}{|l|}{ Gender (base = male) } \\
\hline Female & $\begin{array}{c}3265.22^{* * *} \\
(664.84)\end{array}$ & $\begin{array}{c}5424.93^{* * *} \\
(656.86)\end{array}$ & $\begin{array}{c}26.09^{* * *} \\
(7.42)\end{array}$ & $\begin{array}{c}1.15 \\
(2.54)\end{array}$ \\
\hline \multicolumn{5}{|c|}{ Marital status (base $=$ single) } \\
\hline Married & $\begin{array}{l}1892.40^{*} \\
(744.56)\end{array}$ & $\begin{array}{l}1557.67^{*} \\
(738.85)\end{array}$ & $\begin{array}{c}31.80^{* * *} \\
(8.61)\end{array}$ & $\begin{array}{l}-3.08 \\
(2.82)\end{array}$ \\
\hline Divorced & $\begin{array}{c}3040.23^{* * *} \\
(978.53)\end{array}$ & $\begin{array}{c}3012.25^{* *} \\
(971.98)\end{array}$ & $\begin{array}{l}29.29^{*} \\
(11.37)\end{array}$ & $\begin{array}{l}-5.68 \\
(3.89)\end{array}$ \\
\hline Widowed & $\begin{array}{c}-782.15 \\
(2200.31)\end{array}$ & $\begin{array}{l}-1036.06 \\
(2153.15)\end{array}$ & $\begin{array}{c}2.63 \\
(24.04)\end{array}$ & $\begin{array}{c}5.30 \\
(8.34)\end{array}$ \\
\hline \multicolumn{5}{|c|}{ Education Level (base = no high school no apprenticeship) } \\
\hline $\begin{array}{l}\text { No high school } \\
\text { with an } \\
\text { apprenticeship }\end{array}$ & $\begin{array}{c}1284.97 \\
(1115.22)\end{array}$ & $\begin{array}{c}449.13 \\
(1115.56)\end{array}$ & $\begin{array}{c}20.73 \\
(15.30)\end{array}$ & $\begin{array}{l}-3.37 \\
(5.48)\end{array}$ \\
\hline $\begin{array}{l}\text { High school no } \\
\text { apprenticeship }\end{array}$ & $\begin{array}{c}13837.49^{* * *} \\
(3230.30)\end{array}$ & $\begin{array}{c}11175.57^{* * *} \\
(3183.65)\end{array}$ & $\begin{array}{c}136.79^{* * *} \\
(33.38)\end{array}$ & $\begin{array}{l}-15.41 \\
(10.47)\end{array}$ \\
\hline $\begin{array}{l}\text { High school with an } \\
\text { apprenticeship }\end{array}$ & $\begin{array}{l}4193.55^{* *} \\
(1595.49)\end{array}$ & $\begin{array}{c}1993.62 \\
(1582.18)\end{array}$ & $\begin{array}{l}39.51^{*} \\
(18.60)\end{array}$ & $\begin{array}{l}-1.31 \\
(6.24)\end{array}$ \\
\hline
\end{tabular}




\begin{tabular}{|c|c|c|c|c|}
\hline Variable & $\begin{array}{c}\text { Nominal } \\
\text { Income }(€)\end{array}$ & $\begin{array}{c}\text { Real } \\
\text { Income }(€)\end{array}$ & $\begin{array}{l}\text { Employed } \\
\text { (in days) }\end{array}$ & $\begin{array}{l}\text { Short-term } \\
\text { UE (in days) }\end{array}$ \\
\hline Technical College & $\begin{array}{c}17538.87^{* * *} \\
(1737.22)\end{array}$ & $\begin{array}{c}14733.7^{* * *} \\
(1730.79)\end{array}$ & $\begin{array}{c}187.83^{* * *} \\
(21.08)\end{array}$ & $\begin{array}{c}-21.38^{* * *} \\
(6.42)\end{array}$ \\
\hline University degree & $\begin{array}{c}7353.93^{* * *} \\
(1768.19\end{array}$ & $\begin{array}{l}3894.37^{*} \\
(1749.12\end{array}$ & $\begin{array}{l}45.89^{*} \\
(18.79)\end{array}$ & $\begin{array}{l}-10.75 \\
(6.84)\end{array}$ \\
\hline \multicolumn{5}{|c|}{ Job type (base = unskilled blue-collar) } \\
\hline $\begin{array}{l}\text { Low skilled blue- } \\
\text { collar }\end{array}$ & $\begin{array}{l}-3420.75 \\
(1958.88)\end{array}$ & $\begin{array}{l}-3325.67 \\
(1971.89)\end{array}$ & $\begin{array}{l}-15.86 \\
(29.27)\end{array}$ & $\begin{array}{l}-17.12 \\
(9.77)\end{array}$ \\
\hline Skilled blue-collar & $\begin{array}{c}-5327.16^{* * *} \\
(1550.49)\end{array}$ & $\begin{array}{c}-5047.20^{* *} \\
(1562.13)\end{array}$ & $\begin{array}{c}6.28 \\
(22.46)\end{array}$ & $\begin{array}{l}-24.17^{* *} \\
(7.50)\end{array}$ \\
\hline Master / Craftsmen & $\begin{array}{l}-1939.87 \\
(3884.69)\end{array}$ & $\begin{array}{l}-4751.52 \\
(3841.81)\end{array}$ & $\begin{array}{l}95.66^{*} \\
(39.54)\end{array}$ & $\begin{array}{l}-34.08^{*} \\
(13.85)\end{array}$ \\
\hline White-collar & $\begin{array}{l}5084.77^{* *} \\
(1636.37)\end{array}$ & $\begin{array}{c}2411.68 \\
(1640.49)\end{array}$ & $\begin{array}{c}130.68^{* * *} \\
(23.13)\end{array}$ & $\begin{array}{c}-29.24^{* * *} \\
(7.68)\end{array}$ \\
\hline $\begin{array}{l}\text { Self Employed / } \\
\text { Civil Service }\end{array}$ & $\begin{array}{l}13693.58^{*} \\
(5445.12)\end{array}$ & $\begin{array}{c}16422.36^{* *} \\
(5368.99)\end{array}$ & $\begin{array}{c}294.63^{* * *} \\
(59.35)\end{array}$ & $\begin{array}{l}-49.35^{*} \\
(21.93)\end{array}$ \\
\hline \multicolumn{5}{|c|}{ Employment status (base $=$ full-time work) } \\
\hline $\begin{array}{l}\text { Full time piece } \\
\text { work }\end{array}$ & $\begin{array}{c}-6951.71^{* * *} \\
(1281.49)\end{array}$ & $\begin{array}{c}-6466.60^{* * *} \\
(1263.00)\end{array}$ & $\begin{array}{l}-31.84^{*} \\
(14.71)\end{array}$ & $\begin{array}{c}18.99^{* * *} \\
(5.23)\end{array}$ \\
\hline $\begin{array}{l}\text { Fulltime with night- } \\
\text { shifts }\end{array}$ & $\begin{array}{c}-10225.80^{* * *} \\
(1989.10)\end{array}$ & $\begin{array}{c}-9995.74^{* * *} \\
(1967.63)\end{array}$ & $\begin{array}{l}-42.71 \\
(21.83)\end{array}$ & $\begin{array}{l}22.19^{* *} \\
(7.49)\end{array}$ \\
\hline $\begin{array}{l}\text { Part time work less } \\
\text { than } 50 \%\end{array}$ & $\begin{array}{l}9631.15^{* * *} \\
(1962.84)\end{array}$ & $\begin{array}{c}13866.37^{* * *} \\
(1934.37)\end{array}$ & $\begin{array}{c}138.99^{* * *} \\
(31.69)\end{array}$ & $\begin{array}{l}-4.04 \\
(8.07)\end{array}$ \\
\hline $\begin{array}{l}\text { Part time work } \\
\text { more than } 50 \%\end{array}$ & $\begin{array}{c}3162.22^{* * *} \\
(755.11)\end{array}$ & $\begin{array}{c}6153.77^{* * *} \\
(744.99)\end{array}$ & $\begin{array}{l}28.71^{* *} \\
(9.15)\end{array}$ & $\begin{array}{c}3.67 \\
(2.80)\end{array}$ \\
\hline Other & $\begin{array}{c}5509.45 \\
(4017.91)\end{array}$ & $\begin{array}{c}6825.46 \\
(4013.15)\end{array}$ & $\begin{array}{l}147.81^{*} \\
(58.88)\end{array}$ & $\begin{array}{l}-25.83 \\
(14.44)\end{array}$ \\
\hline Unemployed & $\begin{array}{c}17758.55^{* * *} \\
(1092.45)\end{array}$ & $\begin{array}{c}19823.32^{* * *} \\
(1098.06)\end{array}$ & $\begin{array}{c}286.21^{* * *} \\
(15.08)\end{array}$ & $\begin{array}{c}-111.67^{* * *} \\
(5.26)\end{array}$ \\
\hline $\mathrm{n}$ & 19,250 & 19,250 & 19,250 & 19,250 \\
\hline r2 & 0.31 & 0.29 & 0.32 & 0.10 \\
\hline r2_a & 0.30 & 0.28 & 0.31 & 0.09 \\
\hline
\end{tabular}

Notes: Dependent Variables $=$ Difference between employment outcomes four years after minus four years before admission into vocational rehabilitation. Standard errors in brackets. The standard error is an estimate of the standard deviation of the estimated coefficient; the amount it varies across cases. Other covariates included medical diagnosis, nationality, residential region, year of rehabilitation start. ${ }^{*} \mathrm{p}<.05 ;{ }^{* *} \mathrm{p}<.01 ;{ }^{* *} \mathrm{p}<.001$. 


\section{Correlation of change score with pre-rehabilitation income}

Analysis using a simple gain score does not control for baseline imbalance in the dependent variable and thus might yield undesirable results. This effect is due to baseline values being negatively correlated with change since rehabilitants with low scores at baseline generally improve more than those with high scores. To test whether the results were influenced by the relative starting positions, the attained individual gain scores were graphed against the income in the four years before rehabilitation. Figure 1 visualizes how, with higher pre-rehabilitation income, the gain score of the individual rehabilitants was more negative.

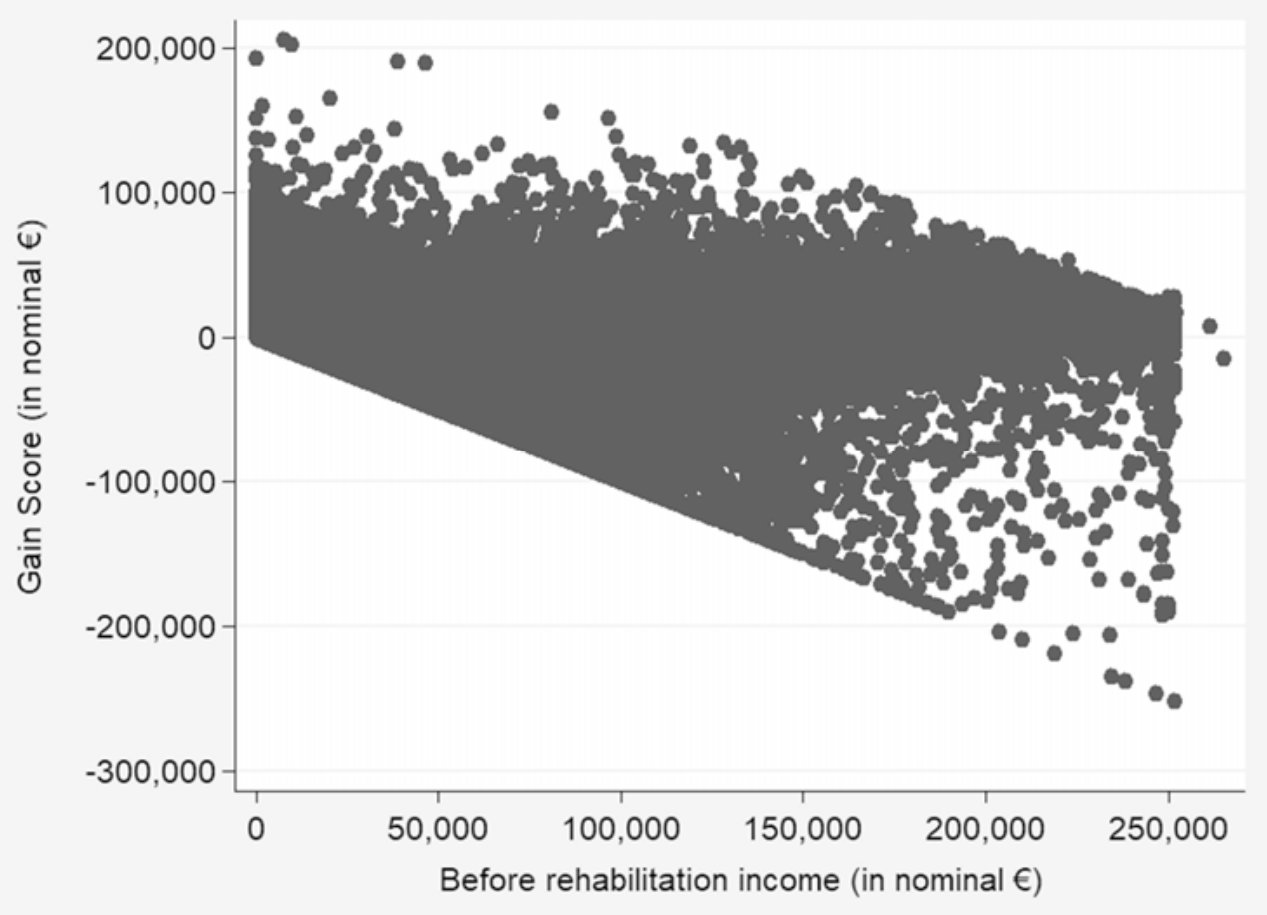

Figure 1 Graphical analysis of before rehabilitation income and gain score

Table 6 shows the extent to which the before-rehabilitation income differed between socio-economic subgroups. Overall, rehabilitant heterogeneity at baseline was high. According to the regression estimate, $50 \%$ of the variation in before rehabilitation earnings was explained based on the included socio-economic characteristics. A greater age was associated with higher earnings while females earned significantly less than males. Married rehabilitants earned significantly more than singles. Higher education was associated with higher earnings, white-collar workers earned substantially more than blue-collar workers, and regular full-time workers had a higher pre-treatment income than persons in all other types of employment. 


\begin{tabular}{|c|c|c|}
\hline Variable & Coef. & SE \\
\hline \multicolumn{3}{|l|}{ Age $($ base $=20-25)$} \\
\hline $26-30$ & $8301.68^{*}$ & $(4128.09)$ \\
\hline $31-35$ & $25306.84^{* * *}$ & $(3825.78)$ \\
\hline $36-40$ & $31682.95^{* * *}$ & $(3674.20)$ \\
\hline $41-45$ & $39657.66^{* * *}$ & $(3651.82)$ \\
\hline $46-50$ & $42799.97^{* * *}$ & $(3660.52)$ \\
\hline $51-55$ & $46974.31^{* * *}$ & $(3682.98)$ \\
\hline $56-60$ & $50476.80^{* * *}$ & $(3769.23)$ \\
\hline $61-62$ & $62311.29^{* * *}$ & $(6166.94)$ \\
\hline \multicolumn{3}{|l|}{ Gender $($ base $=$ male $)$} \\
\hline Female & $-31445.19^{* * *}$ & (771.15) \\
\hline \multicolumn{3}{|l|}{ Marital status (base $=$ single) } \\
\hline Married & $6833.25^{* * *}$ & $(883.68)$ \\
\hline Divorced & -949.21 & $(1170.54)$ \\
\hline Widowed & 5226.90 & $(2804.45)$ \\
\hline \multicolumn{3}{|c|}{ Education Level (base $=$ no high school no apprenticeship) } \\
\hline No high school with an apprenticeship & $10774.38^{* * *}$ & $(1350.30)$ \\
\hline High school no apprenticeship & $30923.52^{* * *}$ & $(3412.79)$ \\
\hline High school with an apprenticeship & $28297.13^{* * *}$ & $(1934.90)$ \\
\hline Technical College & $7206.89^{* * *}$ & $(1642.66)$ \\
\hline University degree & $39411.62^{* * *}$ & $(2019.94)$ \\
\hline \multicolumn{3}{|l|}{ Job type (base = unskilled blue) } \\
\hline Low skilled blue-collar & $5227.33^{*}$ & $(2284.64)$ \\
\hline Skilled blue-collar & -1176.86 & $(1760.09)$ \\
\hline Master / Craftsmen & $56575.16^{* * *}$ & $(3806.92)$ \\
\hline White-collar & $62116.34^{* * *}$ & $(1711.16)$ \\
\hline Self Employed / Civil Service & $-26328.40^{* * *}$ & $(5339.51)$ \\
\hline \multicolumn{3}{|l|}{ Employment status (base $=$ full-time work) } \\
\hline Full-time piece work & $-20545.87^{* * *}$ & $(1361.18)$ \\
\hline Fulltime with nightshifts & $-19098.40^{* * *}$ & $(2063.82)$ \\
\hline Part time work less than $50 \%$ & $-69439.02^{* * *}$ & $(2652.06)$ \\
\hline Part time work more than $50 \%$ & $-44459.67^{* * *}$ & $(1016.53)$ \\
\hline Other & $-29214.58^{* * *}$ & $(4962.78)$ \\
\hline Unemployed & $-60854.23^{* * *}$ & $(1115.62)$ \\
\hline $\mathrm{n}$ & 19,250 & \\
\hline r2 / r2_a & $0.50 / 0.50$ & \\
\hline
\end{tabular}

Notes: Dependent variable $=$ Four-year pre-rehabilitation earnings. Other covariates included were nationality and the rehabilitation start year. ${ }^{*} \mathrm{p}<.05 ;{ }^{* *} \mathrm{p}<.01 ;{ }^{* *} \mathrm{p}<.001$. 


\section{Lagged dependent variable regression results}

Because of the rehabilitants' heterogeneity with regards to their past employment history, a lagged dependent variable regression was performed. Using baseline outcomes as covariates result in greater precision and a more informative value with regards to interpreting the model coefficients. Table 7 provides an overview of the lagged dependent variable regression results. Overall, the higher adjusted r-square compared to previous models was the consequence of including employment history as a covariate instead of as part of the dependent variable.

In the estimation of demographic determinants, the specified age groups remained a significant predictor of employment outcomes. The estimate was slightly smaller in absolute size compared to the gain score regression estimate, suggesting that results based on the gain score regression overestimate the effect of age on employment outcomes as some of the effects were actually linked to higher wages (seniority) before participation in the rehabilitation program, everything else being equal.

For the gender effect, it becomes evident that the lower pre-rehab earnings of women in comparison to men were responsible for the positive regression coefficient of females in the gain score regression. After controlling for the intergroup baseline outcomes, the coefficient of the regression models changed signs; in the lagged dependent variable regression, the female coefficient was negative, significant at the one-percent level. With regards to the marital status, a significant positive effect for married and divorced participants compared to singles was detected. The results with regards to the education status confirmed the results retrieved through the change score regression. Higher prior educational attainments were an important predictor variable of the employment outcomes after participation in vocational rehabilitation.

With regards to the employment type, the indicator variables for part-time work changed from positive and significant, to negative and significant when taking prerehabilitation earnings into account. Moreover, piece work and nightshifts were found to have a negative effect on the level of earnings after rehabilitation. Additionally, the lagged dependent variable estimates confirmed that white-collar workers exhibited significantly greater improvements in employment outcomes than blue-collar workers. In addition, the results show that master/craftsmen were able to increase their earnings substantially above the earnings development of similar blue-collar workers. The coefficient for self-employed remained marginally positive but became insignificant through the inclusion of income history as covariate. 
Table 7: OLS regression of socio-economic factors on accumulative employment outcomes over four years after admission into vocational rehabilitation, adjusted for baseline employment outcomes

\begin{tabular}{|c|c|c|c|c|}
\hline Variable & $\begin{array}{c}\text { Nominal } \\
\text { Income }(€)\end{array}$ & $\begin{array}{c}\text { Real } \\
\text { Income }(€)\end{array}$ & $\begin{array}{c}\text { Employed } \\
\text { (in days) }\end{array}$ & $\begin{array}{l}\text { Short-term } \\
\text { UE (in days) }\end{array}$ \\
\hline $\begin{array}{l}\text { Lagged dependent } \\
\text { variable }\end{array}$ & $\begin{array}{l}0.72^{* * *} \\
(0.01)\end{array}$ & $\begin{array}{l}0.67^{* * *} \\
(0.01)\end{array}$ & $\begin{array}{l}0.21^{* * *} \\
(0.01)\end{array}$ & $\begin{array}{l}0.22^{* * *} \\
(0.01)\end{array}$ \\
\hline \multicolumn{5}{|l|}{ Age (base $=20-25)$} \\
\hline $26-30$ & $\begin{array}{l}-6956.56^{*} \\
(2974.46)\end{array}$ & $\begin{array}{l}-6704.52^{*} \\
(2852.55)\end{array}$ & $\begin{array}{l}-30.31 \\
(35.17)\end{array}$ & $\begin{array}{c}8.05 \\
(8.88)\end{array}$ \\
\hline $31-35$ & $\begin{array}{c}-12282.84^{* * *} \\
(2805.27)\end{array}$ & $\begin{array}{c}-11831.05^{* * *} \\
(2690.36)\end{array}$ & $\begin{array}{l}-36.29 \\
(32.61)\end{array}$ & $\begin{array}{l}14.17 \\
(8.03)\end{array}$ \\
\hline $36-40$ & $\begin{array}{c}-9887.48^{* * *} \\
(2649.30)\end{array}$ & $\begin{array}{l}-9550.24^{* * *} \\
(2540.58)\end{array}$ & $\begin{array}{l}-19.39 \\
(31.42)\end{array}$ & $\begin{array}{l}13.52 \\
(7.66)\end{array}$ \\
\hline $41-45$ & $\begin{array}{l}-9546.39^{* * * *} \\
(2642.43)\end{array}$ & $\begin{array}{l}-9213.54^{* * * *} \\
(2534.02)\end{array}$ & $\begin{array}{c}9.48 \\
(31.33)\end{array}$ & $\begin{array}{l}12.54 \\
(7.62)\end{array}$ \\
\hline $46-50$ & $\begin{array}{c}-13552.96^{* * *} \\
(2660.72)\end{array}$ & $\begin{array}{c}-13054.32^{* * *} \\
(2551.71)\end{array}$ & $\begin{array}{l}-13.47 \\
(31.51)\end{array}$ & $\begin{array}{l}17.33^{*} \\
(7.65)\end{array}$ \\
\hline $51-55$ & $\begin{array}{c}-18288.94^{* * *} \\
(2688.91)\end{array}$ & $\begin{array}{c}-17583.44^{* * *} \\
(2578.78)\end{array}$ & $\begin{array}{l}-38.22 \\
(31.70)\end{array}$ & $\begin{array}{l}28.99^{* * *} \\
(7.79)\end{array}$ \\
\hline $56-60$ & $\begin{array}{c}-24565.91^{* * *} \\
(2793.12)\end{array}$ & $\begin{array}{c}-23565.21^{* * *} \\
(2678.45)\end{array}$ & $\begin{array}{l}-75.96^{* *} \\
(32.44)\end{array}$ & $\begin{array}{c}55.50^{* * *} \\
(8.34)\end{array}$ \\
\hline $61-62$ & $\begin{array}{c}-27286.59^{* * *} \\
(4810.67)\end{array}$ & $\begin{array}{c}-25947.12^{* * *} \\
(4602.69)\end{array}$ & $\begin{array}{c}-117.53^{*} \\
(46.58)\end{array}$ & $\begin{array}{c}57.22^{* * *} \\
(18.16)\end{array}$ \\
\hline \multicolumn{5}{|l|}{ Gender $($ base $=$ male $)$} \\
\hline Female & $\begin{array}{c}-4603.44^{* * * *} \\
(667.08)\end{array}$ & $\begin{array}{c}-4456.18^{* * *} \\
(640.26)\end{array}$ & $\begin{array}{c}21.70^{* * *} \\
(6.03)\end{array}$ & $\begin{array}{l}-3.91^{*} \\
(1.81)\end{array}$ \\
\hline \multicolumn{5}{|c|}{ Marital status (base $=$ single) } \\
\hline Married & $\begin{array}{c}2796.30^{* * *} \\
(703.96)\end{array}$ & $\begin{array}{c}2692.43^{* * *} \\
(675.83)\end{array}$ & $\begin{array}{c}28.54^{* * *} \\
(6.86)\end{array}$ & $\begin{array}{l}-1.96 \\
(1.98)\end{array}$ \\
\hline Divorced & $\begin{array}{c}2767.09^{* *} \\
(918.86)\end{array}$ & $\begin{array}{l}2662.03^{*} \\
(882.06)\end{array}$ & $\begin{array}{l}1.10 \\
(9.15)\end{array}$ & $\begin{array}{c}0.02 \\
(2.76)\end{array}$ \\
\hline Widowed & $\begin{array}{c}287.25 \\
(2161.95)\end{array}$ & $\begin{array}{c}307.03 \\
(2073.64)\end{array}$ & $\begin{array}{c}19.08 \\
(21.39)\end{array}$ & $\begin{array}{c}0.03 \\
(6.31)\end{array}$ \\
\hline \multicolumn{5}{|c|}{ Education Level (base $=$ no high school no apprenticeship) } \\
\hline $\begin{array}{l}\text { No high school } \\
\text { with apprenticeship }\end{array}$ & $\begin{array}{l}3925.45^{* * *} \\
(1017.26)\end{array}$ & $\begin{array}{c}3764.96^{* * *} \\
(976.97)\end{array}$ & $\begin{array}{l}72.45^{* * *} \\
(12.22)\end{array}$ & $\begin{array}{l}-1.63 \\
(3.96)\end{array}$ \\
\hline $\begin{array}{l}\text { High school no } \\
\text { apprenticeship }\end{array}$ & $\begin{array}{c}20866.52^{* * *} \\
(3138.45)\end{array}$ & $\begin{array}{c}19991.97^{* * *} \\
(3013.98)\end{array}$ & $\begin{array}{c}205.13^{* * *} \\
(28.57)\end{array}$ & $\begin{array}{c}-17.63^{*} \\
(8.04)\end{array}$ \\
\hline
\end{tabular}




\begin{tabular}{|c|c|c|c|c|}
\hline Variable & $\begin{array}{c}\text { Nominal } \\
\text { Income }(€)\end{array}$ & $\begin{array}{c}\text { Real } \\
\text { Income }(€)\end{array}$ & $\begin{array}{l}\text { Employed } \\
\text { (in days) }\end{array}$ & $\begin{array}{l}\text { Short-term } \\
\text { UE (in days) }\end{array}$ \\
\hline High school with & $11161.60^{* * *}$ & $10737.14^{* * *}$ & $102.73^{* * *}$ & -4.09 \\
\hline apprenticeship & $(1528.83)$ & $(1467.55)$ & $(15.36)$ & $(4.62)$ \\
\hline Technical College & $\begin{array}{c}24603.30^{* * *} \\
(1624.39)\end{array}$ & $\begin{array}{c}23596.32^{* * *} \\
(1559.25)\end{array}$ & $\begin{array}{c}225.10^{* * *} \\
(16.66)\end{array}$ & $\begin{array}{c}-20.87^{* * *} \\
(4.55)\end{array}$ \\
\hline University degree & $\begin{array}{c}18239.84^{* * *} \\
(1726.16)\end{array}$ & $\begin{array}{c}17558.46^{* * *} \\
(1656.19)\end{array}$ & $\begin{array}{l}93.93^{* * *} \\
(15.59)\end{array}$ & $\begin{array}{l}-7.73 \\
(5.00)\end{array}$ \\
\hline \multicolumn{5}{|c|}{ Job type (base = unskilled blue-collar) } \\
\hline $\begin{array}{l}\text { Low skilled blue- } \\
\text { collar }\end{array}$ & $\begin{array}{l}-3400.37^{*} \\
(1725.86)\end{array}$ & $\begin{array}{l}-3283.40 \\
(1658.94)\end{array}$ & $\begin{array}{c}-0.90 \\
(21.01)\end{array}$ & $\begin{array}{c}3.96 \\
(6.48)\end{array}$ \\
\hline Skilled blue-collar & $\begin{array}{c}-5802.60^{* * *} \\
(1359.44)\end{array}$ & $\begin{array}{c}-5624.95^{* * *} \\
(1306.67)\end{array}$ & $\begin{array}{l}-13.64 \\
(15.99)\end{array}$ & $\begin{array}{c}0.30 \\
(4.99)\end{array}$ \\
\hline Master / Craftsmen & $\begin{array}{l}7449.31^{*} \\
(3652.31)\end{array}$ & $\begin{array}{l}7058.43^{*} \\
(3505.86)\end{array}$ & $\begin{array}{c}190.88^{* * * *} \\
(32.68)\end{array}$ & $\begin{array}{l}-18.01^{*} \\
(10.06)\end{array}$ \\
\hline White-collar & $\begin{array}{c}13030.83^{* * *} \\
(1477.27)\end{array}$ & $\begin{array}{c}12416.90^{* * *} \\
(1419.65)\end{array}$ & $\begin{array}{c}216.70^{* * *} \\
(17.19)\end{array}$ & $\begin{array}{c}-22.77^{* * *} \\
(5.25)\end{array}$ \\
\hline $\begin{array}{l}\text { Self Employed / } \\
\text { Civil Service }\end{array}$ & $\begin{array}{c}1363.46 \\
(5491.88)\end{array}$ & $\begin{array}{c}1002.23 \\
(5290.93)\end{array}$ & $\begin{array}{c}-123.86^{* *} \\
(63.59)\end{array}$ & $\begin{array}{l}-16.85 \\
(15.67)\end{array}$ \\
\hline \multicolumn{5}{|c|}{ Employment status (base $=$ Full-time work) } \\
\hline $\begin{array}{l}\text { Full time piece } \\
\text { work }\end{array}$ & $\begin{array}{c}-7836.73^{* * *} \\
(1226.97)\end{array}$ & $\begin{array}{c}-7569.17^{* * *} \\
(1177.77)\end{array}$ & $\begin{array}{c}2.94 \\
(12.98)\end{array}$ & $\begin{array}{c}11.94^{* * *} \\
(3.92)\end{array}$ \\
\hline $\begin{array}{l}\text { Fulltime with } \\
\text { nightshifts }\end{array}$ & $\begin{array}{c}-10007.63^{* * *} \\
(1862.92)\end{array}$ & $\begin{array}{c}-9700.51^{* * *} \\
(1787.05)\end{array}$ & $\begin{array}{c}8.92 \\
(19.52)\end{array}$ & $\begin{array}{c}7.00 \\
(5.75)\end{array}$ \\
\hline $\begin{array}{l}\text { Part time work less } \\
\text { than } 50 \%\end{array}$ & $\begin{array}{l}-6368.97^{* *} \\
(1969.45)\end{array}$ & $\begin{array}{l}-6231.03^{* *} \\
(1893.04)\end{array}$ & $\begin{array}{l}-13.49 \\
(23.44)\end{array}$ & $\begin{array}{l}-8.74 \\
(5.26)\end{array}$ \\
\hline $\begin{array}{l}\text { Part time work } \\
\text { more than } 50 \%\end{array}$ & $\begin{array}{c}-7711.53^{* * *} \\
(786.49)\end{array}$ & $\begin{array}{c}-7500.07^{* * *} \\
(754.91)\end{array}$ & $\begin{array}{l}17.90^{*} \\
(7.54)\end{array}$ & $\begin{array}{l}-0.98 \\
(2.10)\end{array}$ \\
\hline Other & $\begin{array}{c}132.56 \\
(3675.05)\end{array}$ & $\begin{array}{c}90.77 \\
(3534.69)\end{array}$ & $\begin{array}{l}90.32^{*} \\
(40.76)\end{array}$ & $\begin{array}{l}-2.63 \\
(9.34)\end{array}$ \\
\hline Unemployed & $\begin{array}{c}8222.43^{* * *} \\
(1001.74)\end{array}$ & $\begin{array}{c}7851.10^{* * *} \\
(962.58)\end{array}$ & $\begin{array}{l}-14.62 \\
(12.11)\end{array}$ & $\begin{array}{c}-38.10^{* * *} \\
(3.58)\end{array}$ \\
\hline $\mathrm{n}$ & 19,250 & 19,250 & 19,250 & 19,250 \\
\hline r2 & 0.78 & 0.78 & 0.62 & 0.25 \\
\hline r2_a & 0.78 & 0.78 & 0.61 & 0.24 \\
\hline
\end{tabular}

Notes: Dependent variable $=$ Four-year post-rehabilitation outcomes. Covariates now include a lagged dependent variable: the sum of employment outcomes in the four years before admission. Other covariates included were the medical diagnosis, nationality, residential region and the year of rehabilitation start. Standard errors in brackets. ${ }^{*} \mathrm{p}<.05 ;{ }^{* *} \mathrm{p}<.01 ;{ }^{* * *} \mathrm{p}<.001$. 


\section{Stratification by gender}

Separate regression results for men and women are presented in Table 8. Overall, the demographic effects were more strongly associated with post-rehabilitation employment outcomes for men than for women. For women, pre-rehabilitation earnings, instead, had a relatively larger effect. Interestingly, while most coefficients between men and women differed only with respect to their size, the coefficients of the factors divorced, widowed and self-employed were of opposite sign.

Table 8: Stratified OLS regression of socio-economic factors on employment outcomes (by gender)

\begin{tabular}{lcc}
\hline Variable & Males & Females \\
\hline Lagged dependent variable (pre- & $0.65^{* * *}$ & $0.76^{* * *}$ \\
rehabilitation earnings) & $(0.01)$ & $(0.01)$ \\
Age (base = 20-25) & & \\
$26-30$ & -7049.31 & $-7628.87^{*}$ \\
& $(5682.22)$ & $(3799.23)$ \\
$31-35$ & $-14179.16^{* *}$ & $-10645.96^{* *}$ \\
& $(5310.84)$ & $(3557.05)$ \\
$36-40$ & $-16303.46^{* *}$ & -4442.88 \\
& $(5178.18)$ & $(3390.60)$ \\
$41-45$ & $-16552.77^{* *}$ & -3338.15 \\
& $(5164.57)$ & $(3375.57)$ \\
$46-50$ & $-20328.64^{* * *}$ & $-7681.88^{*}$ \\
& $(5187.07)$ & $(3397.21)$ \\
$51-55$ & $-25933.59^{* * *}$ & $-11234.53^{* *}$ \\
& $(5231.17)$ & $(3431.56)$ \\
$56-60$ & $-36053.37^{* * *}$ & $-14591.00^{* * *}$ \\
& $(5322.99)$ & $(3531.86)$ \\
$61-62$ & $-39511.31^{* * *}$ & $-17266.83^{* *}$ \\
& $(7600.47)$ & $(6450.67)$ \\
Marital status (base = single) & & \\
Married & $3244.58^{* *}$ & $2251.81^{*}$ \\
Divorced & $(1111.70)$ & $(912.80)$ \\
Widowed & -505.26 & $3591.81^{* * *}$ \\
No high school with apprenticeship & $(1631.82)$ & $(1086.17)$ \\
& -10134.16 & 747.38 \\
& $(5648.10)$ & $(2259.57)$ \\
& $(1579.27)$ & $(1444.82)$ \\
& & \\
& & \\
& &
\end{tabular}




\begin{tabular}{|c|c|c|}
\hline Variable & Males & Females \\
\hline High school no apprenticeship & $\begin{array}{c}23912.65^{* * *} \\
(3797.37)\end{array}$ & $\begin{array}{c}18316.62^{* * *} \\
(3810.05)\end{array}$ \\
\hline High school with apprenticeship & $\begin{array}{c}15180.64^{* * *} \\
(2465.46)\end{array}$ & $\begin{array}{c}9600.43^{* * *} \\
(1936.47)\end{array}$ \\
\hline Technical College & $\begin{array}{c}28020.92^{* * *} \\
(2230.71)\end{array}$ & $\begin{array}{c}20804.41^{* * *} \\
(2055.63)\end{array}$ \\
\hline University degree & $\begin{array}{c}20931.66^{* * *} \\
(2511.47)\end{array}$ & $\begin{array}{c}16917.19^{* * *} \\
(2115.12)\end{array}$ \\
\hline \multicolumn{3}{|l|}{ Job type (base $=$ unskilled blue-collar) } \\
\hline Low skilled blue-collar & $\begin{array}{l}-3786.39 \\
(2438.13)\end{array}$ & $\begin{array}{l}-2136.20 \\
(2812.22)\end{array}$ \\
\hline Skilled blue-collar & $\begin{array}{l}-3712.76 \\
(1928.62)\end{array}$ & $\begin{array}{l}-1720.31 \\
(2270.34)\end{array}$ \\
\hline Master / Craftsmen & $\begin{array}{c}13828.09^{* * *} \\
(3821.54)\end{array}$ & $\begin{array}{c}850.63 \\
(5805.06)\end{array}$ \\
\hline White-collar & $\begin{array}{c}18165.44^{* * *} \\
(2153.59)\end{array}$ & $\begin{array}{c}7451.55^{* * *} \\
(2121.38)\end{array}$ \\
\hline Self Employed / Civil Service & $\begin{array}{l}-3055.18 \\
(5950.40)\end{array}$ & $\begin{array}{c}5039.12 \\
(6120.55)\end{array}$ \\
\hline \multicolumn{3}{|c|}{ Employment status (base = Full-time work) } \\
\hline Full time piece work & $\begin{array}{c}-4986.75^{* *} \\
(1751.23)\end{array}$ & $\begin{array}{c}-11033.06^{* * *} \\
(1396.83)\end{array}$ \\
\hline Fulltime with nightshifts & $\begin{array}{c}-6813.37^{* *} \\
(2546.98)\end{array}$ & $\begin{array}{c}-12093.13^{* * *} \\
(2140.35)\end{array}$ \\
\hline Part time work less 50 & $\begin{array}{l}-7576.01 \\
(6288.65)\end{array}$ & $\begin{array}{l}-5096.97^{*} \\
(2142.64)\end{array}$ \\
\hline Part time work plus 50 & $\begin{array}{c}-18561.39^{* * *} \\
(2899.88)\end{array}$ & $\begin{array}{c}-5519.37^{* * *} \\
(868.50)\end{array}$ \\
\hline Other & $\begin{array}{c}5820.16 \\
(5434.31)\end{array}$ & $\begin{array}{l}-7348.97 \\
(5711.25)\end{array}$ \\
\hline Unemployed & $\begin{array}{c}11748.56^{* * *} \\
(1355.90)\end{array}$ & $\begin{array}{c}2825.79 \\
(1484.12)\end{array}$ \\
\hline $\mathrm{n}$ & 8,856 & 10,394 \\
\hline $\mathrm{r} 2 / \mathrm{r} 2 \mathrm{a}$ & $0.82 / 0.82$ & $0.74 / 0.73$ \\
\hline
\end{tabular}

Notes: Dependent variable $=$ Four-year post-rehabilitation earnings. Standard errors in brackets. Other covariates included were medical diagnosis, nationality, region and the year of rehabilitation start. Standard errors in brackets. ${ }^{*} \mathrm{p}<.05 ;{ }^{* *} \mathrm{p}<.01 ;{ }^{* * *} \mathrm{p}<.001$ 


\subsection{Discussion}

In this chapter, employment outcomes and their determinants have been investigated through an analysis of representative panel data of German rehabilitants. The focus on rehabilitant-level characteristics in this chapter has only received limited attention in international research as microdata in the form used here is rarely available. As such, this chapter provides nationally and internationally new and relevant insights with regards to the effects of demographic factors and job characteristics on labor market outcomes before and after participation in vocational rehabilitation.

First, using income and employment data for several years before and after program entry, cohort and individual employment results were measured. Second, it was examined to what extent differences in return-to-work outcomes among rehabilitants can be linked to their socio-economic status. The outcome variables of interest were income from labor, days with employment and days with short-term unemployment benefits in the first four years after admission into a vocational rehabilitation program.

In the beginning of the results section, it was shown that program participants on average experienced a drop in their earnings and more days with unemployment in the years before admission into rehabilitation. After admission, earnings started to rise again until nominal levels were, on average, equal to the pre-treatment level of income. According to the analysis of socio-economic predictors, before-after differences in the employment outcomes can be predicted to a substantial extent based on the participants' socio-demographic and employment characteristics.

Among the different factors tested, the personal employment and income history were the strongest predictors of future labor market outcomes. Moreover, it has been shown that the rehabilitants' ages, educations, jobs and employment types had a large and significant effect on employment outcomes after vocational rehabilitation. Younger, better educated, white-collar workers and those employed in full-time contracts exhibited significantly higher changes in labor market outcomes than their respective comparison groups.

Differences in employment outcomes with regards to gender and marital status were, in comparison, rather small, but also significant. To provide more detailed results with regards to gender-specific effects, the analysis was extended by a stratified regression model showing gender-specific effects of socio-economic factors. Most notably, the coefficients for being widowed, divorced or self-employed were different for men and women. 
Among the group of rehabilitants evaluated, there were a comparably high number of rehabilitants with low variation in pre-post results, which, to some extent, can be attributed to workers who were unemployed in the years before the measures and who remain unemployed in the years after. As a result, the estimated differences for those that do experience a change in their working status are likely to be larger.

The relatively good fit of the change score regression models in comparison to that of a similar study based on medical rehabilitation data from the same source (Petrunyk, Pfeifer, Fischer, \& Wiemer, 2015) can, to some extent, be linked to the more cohesive data and a longer time horizon. Another explanation could be that employment outcomes after vocational rehabilitation are more dependent on socioeconomic characteristics than are employment outcomes after medical rehabilitation.

The first takeaway from this analysis pertains to the ability to collect and analyze large administrative datasets with substantial observation periods before and after participation in vocational rehabilitation in Germany. The analysis shows that a rehabilitant's socio-economic status plays a substantial role in the return to work of that individual. The findings support the argument that providers of vocational rehabilitation services need to be aware of the influence demographic and occupational factors have on labor market outcomes.

The results are, however, not suitable to be directly used in allocating vocational rehabilitation treatments. Rather, they point to potential problems in the screening mechanisms, which should be reconsidered; potential problems during the period of treatment and general reintegration problems, which could stem from discrimination in the labor market, incentives and disincentives provided by the retirement and unemployment benefit system. Further case studies and econometric analyses are needed to evaluate, and consequently provide concrete answers to these potential problems. 

3. The impact of vocational re-training on employment outcomes 


\subsection{Introduction}

Although it has been shown that there are many benefits to hiring people with disabilities (Lindsay et al., 2018), people with a disability still face considerable economic disadvantages compared to working-age people without disabilities. Disadvantages include lower employment rates and a significantly higher risk of living in poverty (OECD, 2010; World Health Organisation \& The World Bank, 2011). Other consequences of prolonged unemployment include a lower quality of life and reduced social inclusion. The situation also constitutes a major public concern since low employment rates among people with disabilities are in many ways a challenge to economic productivity and the financial stability of social security systems (OECD, 2009).

These factors have led to numerous occupational rehabilitation studies, some of them examining the effectiveness of interventions promoting re-employment. However, whereas systematic evidence shows how interventions implemented at the workplace impact employment outcomes (Cullen et al., 2018; Franche et al., 2005; Furlan et al., 2012; Hoosain et al., 2019; McDowell \& Fossey, 2015; Nevala et al., 2015; Odeen et al., 2013; van Vilsteren et al., 2015), there is less conclusive evidence on the effects of (out-of-job) re-training measures. Some empirical studies indicate a positive effect on income and employment (Campolieti et al., 2014; D. Dean et al., 2015; Laaksonen \& Gould, 2015) while other authors find little or no effects resulting from program participation (Aakvik, 2003; Frölich et al., 2004). Interpreting these findings is complicated by differences in study populations, methods used and in the vocational education measures analyzed, which may have different mandates, strategies and curricula.

A common problem throughout the analysis of vocational re-training measures is that researchers generally struggle to recruit study participants, which may limit the types and appropriateness of methods being used (Lysaght et al., 2016). Due to their individual health situation, people with a disability are a heterogeneous group, making it difficult to find suitable comparison groups for program evaluation (Sears et al., 2014). Individuals who are deemed eligible to participate in rehabilitation measures usually cannot be denied access to the services. The challenge in evaluating the impact of an intervention is thus to obtain a credible estimate on the counterfactual: What would have happened to the participants of the re-training programs had they not completed the measures?

In the absence of a natural comparison group, an alternative is to draw a comparison with program applicants who were admitted into a re-training program but never received the actual benefit from training (due to no-show or dropout). Drawing 
comparison with an applicant-based comparison group has several advantages compared to impact analysis based on an external comparison group design. While program graduates and dropouts share the same motivation to apply for vocational education, satisfy the eligibility criteria, and potentially have similar health problems, selection bias is minimized (D. H. Dean \& Dolan, 1991). Moreover, if data is process generated, distortions due to response denials or omissions in the retrospective collection of data can be dismissed.

Using data from Germany as a case study, this chapter provides new and unique evidence on the effects of vocational re-training on long-term employment outcomes. By drawing a comparison with a group of similar program dropouts, the results of this analysis add to a continuing methodological debate on how to estimate program effects from observational data. A successful outcome of this debate could improve public policymaking. While adjusting the results for measured confounders, it is shown to what extent completion of a re-training program influences subsequent income and employment development. Additionally, the influence of the re-training programs on the number of days with unemployment and social security benefits and with regards to the uptake of a pension due to a limited earnings capacity is evaluated.

The remainder of this chapter is structured as follows: In the next section, the methods are presented, with a description of the study design, the study population, the re-training programs, and the data used. Moreover, the method of statistical analysis is summarized. In the results section, descriptive statistics of the participants are presented and the balance in covariates between comparison groups is evaluated before treatment effects of the re-training measures are presented. In the final section of this chapter implications and shortcomings of the findings are discussed before the conclusions of the research are offered.

\subsection{Methods}

\subsubsection{Study design and population}

To investigate the employment effects associated with vocational re-training in Germany, a retro-perspective, quasi-experimental, cohort study was performed. The impact of the re-training measures was assessed by estimating average treatment effects in comparison to a no-training scenario. In the absence of a natural comparison group, an internal comparison group design was used to compare outcomes with and without vocational re-training. The data used for this analysis was collected from the administrative data records of the German Statutory Pension Insurance Fund (Deutsche Rentenversicherung Bund). They featured accurate, processgenerated information on the earnings development and insurance relationship for 
a cohort of rehabilitants in the eight years after admission into re-training and two years prior.

The study population consisted of people with disabilities who, due to their health problem, were no longer able to (or were it was predicted that they will in the foreseeable future no longer be able to) carry out prior job tasks. Additionally, they must have successfully applied for vocational rehabilitation with the German Statutory Pension Insurance Fund.

To become eligible for vocational re-training with the German Statutory Pension Insurance Fund, candidates must satisfy any of the following eligibility criteria: Either the applicants 1 ) already receive a pension due to limited earnings capacity, or 2) an assessment has been made that, without the measure, the pension provider would have to pay out a pension due to limited earnings capacity of the person with a disability, or 3) a medical rehabilitation alone is determined to be insufficient for proper reintegration of a person with a disability into the labor market or 4) the 15year waiting period has been completed.

Additionally, the training scheme needs to be considered necessary for the rehabilitant and there should be a positive chance of a successful measure and consequent re-employment. This success is, for instance, likely if the chances of employment in the rehabilitants' target occupation are good but require additional skills or job-specific knowledge.

Once the application for rehabilitation has been approved, suitable re-training programs were chosen from a large pool of certified private or public institutions specialized in different professions and skill training. Vocational re-training in Germany can generally be categorized into partial and full vocational re-training programs. Apart from the fact that both types of re-training programs require fulltime participation, they differ considerably in contents and length:

- Full re-training programs are comparable to regular apprenticeships programs, typically lasting for two years. During the programs, classroom training is combined with on-the-job training to learn skills and obtain a professional qualification in a new field of work. A formal examination is often set at the end of the programs.

- Partial qualification measures, on the other hand, aim at extending existing competencies with additional skills, e.g. in the fields of business administration or information technology. The measures are usually completed within a year, aiming to reintegrate the participants more quickly into working life. 


\subsubsection{Data}

The data used for this analysis was retrieved from administrative records made available by the Research Data Centre of the German Statutory Pension Insurance Fund. The corresponding data (SUF_RSDV2013) is representative of the whole rehabilitant population and features accurate, process-generated information on the income and employment development before and after participation in a rehabilitation measure. Additionally, the data can also be linked to personal socio-economic and health information. The retrieved data consisted of four databases: The first database included a random sample of all vocational re-training cases in the year 2005 and a range of variables linked to implementing the rehabilitation programs. Moreover, it provided information on some labor-market-related and personal characteristics at the time of application for treatment. The data included information on the type of granted rehabilitation measure, the rehabilitation start date, the medical discharge diagnosis (ICD), the employment status and the residential region at the time of application as well as an indicator variable reflecting whether the rehabilitant successfully completed the re-training measures.

The second dataset was retrieved from the pension insurance follow-up database. It provided information on the participants' insurance relationships, consisting of observations from 2003-2013, including annual individual income and days of (un-) employment data. It, thus, overcomes the lack of detailed labor market data present in many other studies. In this study, the following variables were used from this file: year, yearly income, annual days of employment, days with short- and long-term unemployment, days with other social-security benefits and the occupational group in which the individuals were working prior to program start. To provide further information on the participants' socio-demographics, a third dataset was merged with the previous files. The latter file included data on the persons' sex, birth and death years, nationalities and highest attained levels of education. Lastly, a fourth dataset provided information on receiving a pension. The data included information on whether a pension due to a reduced earnings capacity was paid.

Figure 2 illustrates the process of sample selection. The unique identifier, present in all four databases used to merge the data, is the variable "case". Overall, there were 4,039 observations in the initial sample with a program start in 2005. Limiting the observations to those, for which the program outcome has been registered, reduced the sample to 3,445 observations. Furthermore, duplicates were removed, which reduced the sample by four observations. Next, demographic data was added for all remaining observations. Given the information retrieved in the demographic data file, persons that passed away during the observation period were dropped from the sample. This further reduced sample size to 3,360 observations. To retrieve 
information on the employment status before and after participation in vocational rehabilitation longitudinal labor market data was then merged with the rehabilitation data. Due to interrupted or missing income and employment records, 961 observations were dropped from the sample. In the last step, data on pension receipt was added for those cases, in which a pension due to a reduced earnings capacity was awarded. The final sample consists of 2,399 individuals with complete employment records from 2003-2013.

\begin{tabular}{|c|}
\hline Vocational re-training with German Pension Insurance Fund in 2005 \\
\hline $\mathrm{n}=4,039$ \\
\hline
\end{tabular}

\begin{tabular}{|c|}
\hline Outcome of re-training measure is registered \\
\hline $\mathrm{n}=3,445(-594)$ \\
\hline 5 \\
\hline Removed duplicate entries \\
\hline $\mathrm{n}=3,441(-4)$ \\
\hline 5 \\
\hline Demographic data is added \\
\hline $\mathrm{n}=3,441(0)$ \\
\hline 5 \\
\hline Removed deceased cases \\
\hline $\mathrm{n}=3,360(-81)$ \\
\hline$<$ \\
\hline Income and employment data is added \\
\hline $\mathrm{n}=2,399(-961)$ \\
\hline 5 \\
\hline Data on earnings incapacity pension is added \\
\hline $\mathrm{n}=2,399(0)$ \\
\hline
\end{tabular}

Figure 2 Sampling process: Participants in vocational re-training

\subsubsection{Comparison groups}

Figure 3 provides an illustration of the three comparison groups. In order to compare the impact of the re-training programs a trivariate indicator variable was created, reflecting whether the rehabilitant has either dropped out of re-training (did not successfully complete the measures; value $=0$ ), successfully completed a one-year re-training (value $=1$ ) or successfully completed a two-year re-training (value $=2$ ). ${ }^{8}$ In total, the group of program dropouts contained 367 observations, while there were 278 cases with successfully completed one-year re-training and 1,754 cases with

${ }^{8}$ The outcome of the vocational re-training measure was identified using the variable "BFEWMS" from the provided dataset. 
successfully completed two-year re-training. The group of program dropouts included both one-year and two-year program dropouts due to the rather small number of controls available.

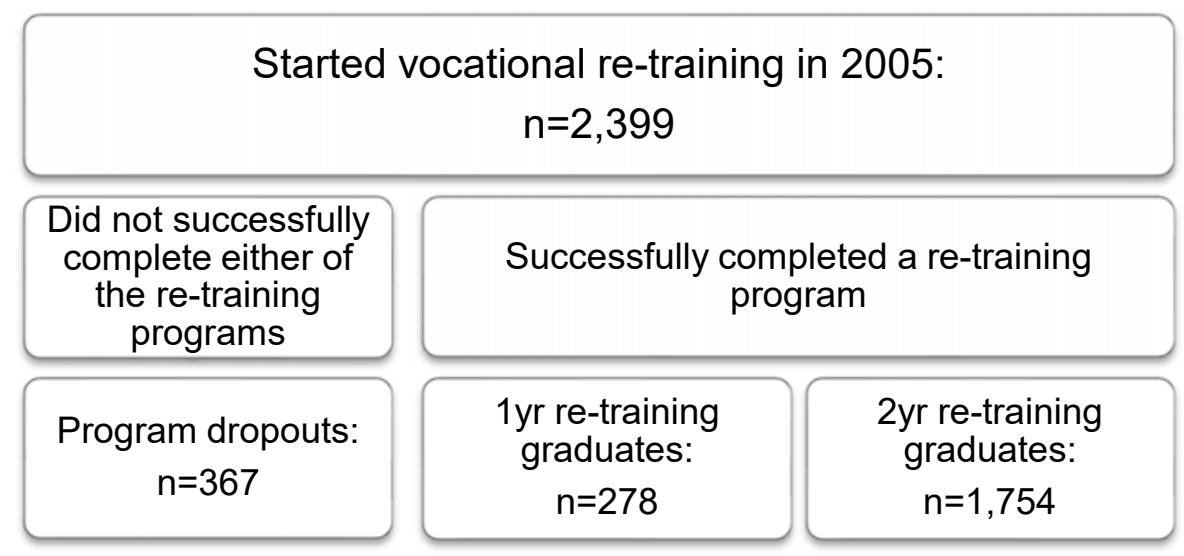

Figure 3 Comparison groups: the impact of vocational re-training

In table 9 the registered reasons for program dropout are listed to provide background information on the causes of unsuccessful program participation. Medical reasons were the predominant reason for program dropout followed by failed examination and other performance issues. Whether this had an influence on the estimations was tested in a sensitivity analysis, which excluded program dropout cases with a registered medical reason for program dropout, from the impact analysis. For the main part of the analysis, all program dropouts $(n=367)$ were used as controls.

Table 9: Registered reasons for program dropout

\begin{tabular}{lll} 
Dropout reason & Freq. & Percent \\
\hline Medical (patient) & 177 & 48.23 \\
Failed Examination & 80 & 21.80 \\
Performance (insurer) & 24 & 6.54 \\
Other (insurer) & 23 & 6.27 \\
Other (patient) & 20 & 5.45 \\
Performance (patient) & 16 & 4.36 \\
Personal (patient) & 15 & 4.09 \\
Disciplinary (insurer) & 9 & 2.45 \\
Economic (patient) & 3 & 0.82
\end{tabular}




\subsubsection{Outcome measures}

Participation in paid employment was assessed using the nominal and real income, as well as employment days, in the first eight years after program admission (20062013). This data was collected directly from the provided pension insurance followup database. A potential drawback of the data used was that employment data collected by the German pension insurance is limited to a contribution ceiling. For annual incomes above $€ 69,600$ ( $€ 58,800$ in the former East German states), the median value of $€ 77,179$ ( $€ 65,400)$ was recorded in the data instead of the true value. This potentially lowers the estimated program impact, as annual income above the specified cut-off value were not properly recorded. Changes in real income were estimated using the average of historical inflation rates collected from the German Federal Bureau of Statistics between 2003 and 2013 (Destatis, 2018). The mean annual discount factor used was equal to $1.6 \%$.

Secondary outcomes evaluated were days on social-security benefits, days on shortterm unemployment benefits, days on long-term unemployment benefits and an indicator variable signaling whether a pension due to a reduced earnings capacity was awarded. The data on social-security benefits included sick pay and temporary allowance payments made during the period of rehabilitation. Short-term unemployment benefits are benefits of the German unemployment insurance, which are paid on the occurrence of unemployment. They are usually paid for up to one year, and for older unemployed people, for up to two years. Long-term unemployment benefits are the basic (means-tested) social security benefits for employable persons in Germany, who are unemployed for longer than 12 months. Data on days with long-term unemployment benefits was only available from 20062009. Workers who are only able to work a few hours a day because of their health can apply for a reduced earnings capacity pension. An individual can claim a reduced earnings capacity pension, if for the foreseeable future, because of ill health or disability, they are unable to do more than three hours of paid work a day.

\section{Missing data analysis}

Before continuing with the description of the independent variables, trends in missing (outcome) data are summarized in this section. In particular, the observations with missing or interrupted income or employment data (961 dropped observations) were closely examined in relation to the uptake of a pension due to a reduced earnings capacity. The results of the analysis were as follows: 1) Among all program dropouts, there was a higher proportion of incomplete income or employment data compared to program graduates (39.14\% vs. 26.30\%). 2) Among observations with incomplete income or employment data, there was a substantially higher proportion of cases with a pension uptake due to a reduced earnings capacity 
(36.54\% vs. 5.42\%). 3) Among program dropout cases with incomplete income or employment data, the proportion of persons receiving a pension due to a reduced earnings capacity was higher compared to graduates with incomplete data. While every second dropout case with missing data was associated with pension uptake, less than every third case in the group of graduates with incomplete data, was associated with pension uptake ( $50 \%$ vs. $32.55 \%)$.

Taken together, the missing data analysis suggests that disproportionally many cases, which were interrupted or incomplete, thus, not considered in our analysis, were cases in which a pension due to a reduced earnings capacity was awarded. There were more dropouts with incomplete data relative to graduates with incomplete data and there was also a higher proportion of awarded earnings incapacity cases in the program dropout group with missing data compared to the graduates with incomplete or missing employment data. This suggests, among other things, that the estimated differences in the forthcoming analysis, i.e. the effects of completing re-training, will be somewhat smaller compared to analysis on the full sample, as missing employment data seems to be positively associated with pension uptake due to a reduced earnings capacity.

The same analysis was also carried out with regards to the uptake of an old-age pension. While the proportion of persons receiving old-age pension was higher in incomplete data cases, the total number was very small, thus, likely only having a small effect on the estimated relationships. Only $0.77 \%$ of all (complete and incomplete) cases received old-age pension between 2006-2013.

\subsubsection{Covariates}

The following variables were included as independent variables in the calculations: age, gender and level of education were collected from the provided demographic database. Additionally, the residential region, the main medical diagnosis and the employment status were retrieved from data collected during the application process. Moreover, based on the information provided from the insurance followup database, several measures of past earnings performance were included. These were the nominal income earned in the year 2003 and 2004 as well as the last registered occupation type.

\subsubsection{Econometric approach}

Adopting the counterfactual framework pioneered by (Rubin, 1974), it was tested whether the re-training measures significantly improved the employment situation compared to the case of no re-training. In this framework a causal effect can be inferred from the difference between two potential outcomes; one that occurs if a person completes a re-training measure, and one that occurs if they do not complete 
the re-training. The following section summarizes the econometric approach used to study the treatment effect associated with vocational re-training in this study. For a more detailed explanation of the methodology used please see the Appendix to Chapter 3.

In this analysis, pre-treatment microdata on the rehabilitants' socio-economic status was used to estimate conditional treatment probabilities (propensity scores). This was done in order to re-weight observations and balance the measured covariates across comparison groups. ${ }^{9}$ Through re-weighting observations, by the inverse probability of being treated, a comparison group with the same distribution of observables as in the treatment group is implicitly created (Hirano \& Imbens, 2001). Because there are a variety of observable factors that can be linked to return-to-work outcomes ${ }^{10}$, it can be cumbersome to determine along which dimension to compare treated and non-treated subjects. Propensity scores, on the other hand, provide a natural weighting scheme that allows the observed differences between comparison groups to be minimized.

\section{Propensity score model}

The developed specification of the propensity score in this analysis included a variety of different variables thought to be characterizing the earnings ability of the rehabilitants. The factors used to predict program allocation/completion can be grouped into individual characteristics, health information, and economic variables on pre-treatment employment outcomes. There is no comprehensive list of variables that would make sure that the matched comparison group can provide an unbiased estimate of the program effect. However, knowledge of the institutional criteria that govern program allocation and the factors that have been found to be strongly correlated to subsequent labor market outcomes, is a good starting point.

One is not guided by the statistical power or significance of the estimated regression coefficients in the selection stage. Rather the objective is to create a sample in which the distribution of covariates that affect labor market outcomes between treated and control subjects is similar, and, thereby, include enough variables for the (weak form of) the conditional independence assumption to hold. This is achieved when adding

\footnotetext{
${ }^{9}$ The original IPW approach was pioneered by Horvitz \& Thompson (Horvitz \& Thompson, 1952). Further important contributions are Robins et al. (Robins, Rotnitzky, \& Zhao, 1995) and Robins \& van der Laan (van der Laan \& Robins, 2003).

10 See e.g. Cornelius et al., 2011; Etuknwa, Daniels, \& Eib, 2019; Langi, Balcazar, \& SuarezBalcazar, 2018, for recent empirical evidence on prognostic individual factors.
} 
additional variables that do not (significantly) alter the estimated coefficients anymore.

Using Statas user-written "bfit" command, combinations of explanatory variables were tested to identify models that exhibit high efficiency based on the combination of covariates included. ${ }^{11}$ The statistical criteria tested to establish efficiency were the Bayesian Information Criterion (BIC) and the Akaike Information Criterion (AIC). Both, the BIC and the AIC, are Penalized-likelihood information criteria that enable cross-comparison of different models using statistical power analysis (Dziak, Coffman, Lanza, \& Runze, 2012). The two information criteria support different models depending on the trade-off between the relative importance one assigns to specificity versus sensitivity.

Using all variables specified in the data section and allowing second-order polynomials as well as interaction terms, all possible combinations of covariates were tested regarding their joint significant and variation explained in relation to the main outcome variable; i.e. the sum of income earned in the years 2006-2013. In accordance with theory, in our estimations, the model with the lowest BIC had a higher joint significance compared to the model with the lowest AIC, an F-statistic of 44.36 vs. 28.91, whereas the model with the lowest AIC was able to explain more of the variation in the dependent variable (Adj. R-squared $=0.2391$ vs. 0.2244 ). All suggested variables to be included according to the BIC and AIC are shown in table 14 in the appendix to chapter 3.

The final specification used in our analysis included all variables suggested by the model with the lowest BIC. Additionally, three further groups of variables were added to the model as suggested by the AIC in order to increase the amount of variation explained. These were an interaction term between before application earnings from 2004 and age at admission, indicator variables reflecting the highest attained level of education and indicator variables for the registered medical diagnosis. Three other terms (Wage2004², Age $^{2}$ and Wage2003*Age), included in the model with the lowest AIC, were not used in the final specification because they correlated strongly with already included variables, while not being existential for the (weak form of the) conditional independence assumption to hold (they did not significantly alter the estimated coefficients when included). Some of the variables, in the final regression model were not individually statistically significant but the likelihood ratio test statistic for the model was large (Prob $>$ chi $2=0.000$ ). The F-

${ }^{11}$ MacDonald, Brodner, \& Jacobs (2004) introduce this technique 
statistic and adjusted variation explained of the final model were in between the models with the optimal BIC and AIC (F-statistic=33.80; Adj. R-squared =0.2366).

The final specification of the propensity score model to estimate the conditional treatment (graduation) probabilities had the following functional form:

$$
\begin{aligned}
\operatorname{Mlogit}(w)=\beta_{0} & +\beta_{1} * \text { Wage } 2003^{2}+\beta_{2} * \text { Wage } 2003+\beta_{3} * \text { Wage } 2004+\beta_{4} * \text { Age } \\
& +\beta_{5} * \text { Wage } 2004 * \text { Age }+\beta_{6} * \text { Working Status }+\beta_{7,8,9 \ldots 15} \\
& * \text { Job Type }+\beta_{16} * \text { Sex }+\beta_{17-20} * \text { Education }+\beta_{21,22} \\
& * \text { ICD diagnosis }+\beta_{23} * \text { Region }+e
\end{aligned}
$$

\section{Outcome Model}

To examine changes in employment outcomes, the average treatment effect (ATE) and the average treatment effect on the treated (ATET), were estimated using the inverse probability weighted regression adjustment method (IPWRA). The IPWRA estimator makes use of conditional probability weights to obtain outcomeregression parameters that account for the missing-data problem, which arises from each subject being observed in only one of the potential outcomes. The IPWRA method is characterized by a three-step procedure to estimate treatment effects:

1) The propensity score was estimated using multinomial logistic regression in order to establish comparability, modeling the program completion status as the dependent variable and individual characteristics as independent variables. The analysis made use of Imbens' generalization of the propensity score (G. W. Imbens, 2000), which showed that the results of Rosenbaum \& Rubin (Rosenbaum \& Rubin, 2006) continue to hold when the treatment is multivalued. ${ }^{12}$ Using Statas' built-in "mlogit" command, conditional treatment probabilities were estimated. The "mlogit" command fits maximum-likelihood multinomial logit models, also known as polytomous logistic regression. ${ }^{13}$ In line with Wooldridge's advice (Wooldridge, Jeffrey, 2010), the specification included all covariates that were correlated with employment outcomes, even though they were not individually significant in the selection model. Following Rosenbaum \& Rubin's approach (Rosenbaum, Paul R, Rubin, 1984), an iterative approach to specifying the propensity score model was used; quadratic- and interaction-terms were added accordingly (as shown in the previous section).

\footnotetext{
${ }^{12}$ See also the papers by Imbens (Imbens, GW, 2004) for further explanations and Cattaneo et al. (Cattaneo, Arbor, Drukker, \& Holland, 2010) or Linden et al (Linden \& Yarnold, 2016) for applications.

${ }^{13}$ For an introduction to multinomial logit models, please see (Greene, 2012; Hosmer, Lemeshow, \& Sturdivant, 2013; Long \& Freese, 2014).
} 
2) Regression models of the employment and social security outcomes were fitted for each treatment level separately (OLS for continuous outcomes, Probit for discrete). ${ }^{14}$ In the regression models, observations were weighted by the inverse probability of treatment (i.e. the conditional probability of people completing a program). The results of the models were used to predict treatment-specific potential outcomes for each subject in both the treated and the non-treated state (of which only one is observed in reality).

3) The means of the treatment-specific outcomes were computed. The differences in these averages provided estimates of the average treatment effects (ATE). In addition, because researchers and policymakers might be more interested in the causal treatment effects only for those who successfully completed the retraining measures, the average treatment effects on the treated (ATET) were calculated. This was achieved by limiting the observations to the subset of graduates before estimating the mean differences between the outcomes with and without program completion.

Following these steps produces consistent estimates of the influence of the intervention because the treatment is assumed to be independent of the potential outcomes after conditioning on the covariates (Cattaneo, 2010). Hereby, it is assumed that all differences between program graduates and non-graduates were due to observable characteristics. The IPWRA estimator exhibits the double robust property meaning that it applies the selection and outcome model simultaneously, thus, producing a consistent estimate of the parameters if either of the two models is correctly specified (Wooldridge, Jeffrey, 2010).

Post-estimation statistics were, henceforth, computed to describe the extent to which covariates were balanced across specifications after the re-weighting of data. In particular, Let $\bar{x}_{g j}$ refer to the sample average of covariate $j$ for the treatment groups $g=0,1,2$ and let $s_{g j}$ be the sample standard deviation; the calculation of normalized differences in a variable then takes the following form (G. W. Imbens \& Rubin, 2015, p. 310):

\footnotetext{
${ }^{14}$ Statistical analysis was performed using the group of "teffects" commands introduced in Stata 13. Compared to the formerly commonly used "psmatch2", the updated algorithm considers the fact that propensity scores are estimated rather than known when calculating standard errors.
} 
The standardized difference in continuous variable $=\frac{\left(\bar{x}_{1 j}-\bar{x}_{0 j}\right)}{\sqrt{\frac{\left(s^{2}{ }_{1 j}+s^{2}{ }_{0 j}\right)}{2}}}$

If the variable was measured on a discrete scale, the standardized differences were calculated as follows:

The standardized difference in discrete variable $=\frac{\left(\hat{p}_{1 j}-\hat{p}_{0 j}\right)}{\sqrt{\frac{\left(\hat{p}_{1 j}\left(1-\hat{p}_{1 j}\right)+\hat{p}_{0 j}\left(1-\hat{p}_{0 j}\right)\right.}{2}}}$

Where $\hat{p}_{1 j}$ and $\hat{p}_{0 j}$ denote the proportion or mean of a binary baseline variable in the treatment and control group, respectively. Although there is no clear cut-off point defined, 0.10 and 0.25 have frequently been considered as values the estimates should be compared against. In general, a difference in average means larger than 0.25 standard deviations is substantial and, in that case, suspicion may be called for (G. Imbens \& Wooldridge, 2007). In contrast, standardized differences as low as 0.10 reflect a degree of balance comparable to what one might expect in a completely randomized experiment (G. W. Imbens \& Rubin, 2015).

\subsection{Results}

\subsubsection{Descriptive characteristics of study participants}

After limiting observations to those for which employment data was recorded for the entire eleven years, the final sample consisted of 2,399 individuals. Of those, 278 had completed a one-year re-training program, 1,754 individuals had successfully completed a two-year re-training program and 367 individuals had not completed either of the re-training programs. Table 10 contains descriptive statistics of the participants at baseline. On average, individuals in the one-year intervention group were older than those in the group of program dropouts, experienced lower pretreatment earnings, were more often unemployed at the time of program application and disproportionally more often came from eastern Germany. The two-year intervention group, on the other hand, was marginally younger than the group of program dropouts, had a relatively higher percentage of females, had higher pretreatment earnings and were less often unemployed at the time of application. The primary medical diagnosis differed only marginally between comparison groups. 
Table 10: Baseline characteristics of the one-year re-training intervention group, the two-year re-training intervention group and the group of program dropouts.

\begin{tabular}{|c|c|c|c|}
\hline Baseline characteristics & $\begin{array}{l}\text { 1-year re- } \\
\text { training } \\
(n=278) \\
\end{array}$ & $\begin{array}{l}2 \text {-year re- } \\
\text { training } \\
(n=1,754) \\
\end{array}$ & $\begin{array}{l}\text { Program } \\
\text { Dropouts } \\
(n=367) \\
\end{array}$ \\
\hline \multicolumn{4}{|l|}{ Age in years } \\
\hline Mean (SD) & $41.58(6.82)$ & $37.87(6.75)$ & $38.11(6.98)$ \\
\hline \multicolumn{4}{|l|}{ Gender } \\
\hline Females & $33 \%$ & $37 \%$ & $31 \%$ \\
\hline $\begin{array}{c}\text { Income 2003, } \\
\text { Mean (SD) }\end{array}$ & $€ 10,096(€ 11,898)$ & $€ 13,249(€ 12,437)$ & $€ 11,078(€ 11,896)$ \\
\hline $\begin{array}{c}\text { Income 2004, } \\
\text { Mean (SD) }\end{array}$ & $€ 5,849(€ 9,280)$ & $€ 6,922(€ 10,115)$ & $€ 5,227(€ 8,991)$ \\
\hline Employment Status & & & \\
\hline Unemployed & $62 \%$ & $44 \%$ & $50 \%$ \\
\hline $\begin{array}{l}\text { Residential Region } \\
\text { Former East } \\
\text { Germany }\end{array}$ & $56 \%$ & $28 \%$ & $30 \%$ \\
\hline $\begin{array}{l}\text { Medical Diagnosis } \\
\text { ICD } 5 \text { (Mental } \\
\text { Disorders) }\end{array}$ & $15 \%$ & $15 \%$ & $13 \%$ \\
\hline ICD 13 (MSD) & $58 \%$ & $64 \%$ & $63 \%$ \\
\hline
\end{tabular}

Notes: Additional control variables used were indicator variables for the level of education and the last registered employment type. For both groups of variables, there were many observations with missing data. Most individuals in the sample for whom education data was available have no tertiary education, but many have completed an apprenticeship. The individuals in the sample for whom data on the employment type was available were most frequently formerly employed in qualified manual tasks, (semi-) professions, simple manual labor and services.

\subsubsection{Covariate balance}

Table 11 shows that the mean standardized differences in covariates between retraining graduates and program dropouts, after re-weighting of observations, were small for all measured baseline characteristics. The differences can be considered negligible when compared to a cut-off of 0.10 standard deviations. The largest improvement in covariate balance, between the initial sample and the weighted sample, was attained by reducing the difference in the share of persons from former east German states between the one-year graduates and the dropout group from 0.53 standard deviations before re-weighting of observations, down to 0.01 standard deviations after re-weighting. Similarly, the difference in income earned in the year 2004 between the two-year graduates and the dropout group was reduced from 0.18 
standard deviations before re-weighting of observations, to 0.01 standard deviations after re-weighting. Summarizing, the analysis of standardized differences suggested that the distribution of covariates between comparison groups were balanced after observations were re-weighted by the inverse probability of being treated.

Table 11: Standardized differences before and after re-weighting observations, one-year re-training intervention group vs. control group and two-year retraining intervention group vs. control group

\begin{tabular}{|c|c|c|c|c|}
\hline & \multicolumn{2}{|c|}{ Initial sample } & \multicolumn{2}{|c|}{ Weighted Sample } \\
\hline Program Dropouts & \multicolumn{2}{|c|}{$n=367$} & \multicolumn{2}{|c|}{$n=794$} \\
\hline 1yr graduates & \multicolumn{2}{|c|}{$n=278$} & \multicolumn{2}{|c|}{$n=809$} \\
\hline 2yr graduates & \multicolumn{2}{|c|}{$n=1,754$} & \multicolumn{2}{|c|}{$n=795$} \\
\hline Baseline characteristics & $1 \mathrm{vs} 0$ & 2vs0 & $1 \mathrm{vs} 0$ & $2 \mathrm{vs} 0$ \\
\hline Age & 0.50 & -0.04 & -0.07 & $<0.01$ \\
\hline Female & 0.04 & 0.13 & 0.04 & 0.02 \\
\hline Income 2003 & -0.08 & 0.18 & -0.06 & 0.01 \\
\hline Income 2004 & 0.07 & 0.18 & 0.05 & $<0.01$ \\
\hline Income $2004 *$ Age & 0.12 & 0.17 & 0.04 & $<0.01$ \\
\hline Income $2003 *$ Income 2003 & -0.05 & 0.16 & -0.01 & 0.01 \\
\hline Unemployed & 0.26 & -0.12 & -0.10 & $<0.01$ \\
\hline Former East Germany & 0.53 & -0.05 & $<0.01$ & 0.01 \\
\hline ICD 5 (Mental Disorders) & 0.08 & 0.06 & $<0.01$ & -0.02 \\
\hline ICD 13 (MSD) & -0.12 & $<0.01$ & -0.07 & 0.03 \\
\hline Neither Abitur nor apprenticeship & -0.04 & -0.11 & -0.03 & $<0.01$ \\
\hline No Abitur but apprenticeship & -0.08 & 0.13 & 0.09 & 0.00 \\
\hline Abitur & -0.04 & -0.02 & 0.06 & 0.01 \\
\hline University Degree & -0.06 & -0.14 & 0.06 & $<0.01$ \\
\hline Agriculture & 0.08 & 0.01 & 0.01 & $<0.01$ \\
\hline Simple Manual Labor & 0.07 & 0.03 & -0.11 & -0.01 \\
\hline Qualified Manual Labor & -0.13 & 0.01 & -0.10 & $<0.01$ \\
\hline Technician/Engineer & 0.07 & -0.03 & -0.01 & -0.04 \\
\hline Simple Services & -0.02 & -0.06 & -0.07 & $<0.01$ \\
\hline Qualified Services & -0.11 & -0.02 & -0.06 & 0.01 \\
\hline (Semi-) Professions & -0.12 & 0.14 & 0.06 & 0.02 \\
\hline Simple commercial/administrative & -0.08 & -0.07 & 0.04 & $<0.01$ \\
\hline Qualified commercial/administrative & 0.04 & 0.03 & -0.02 & $<0.01$ \\
\hline
\end{tabular}

Notes: Initial Sample, standardized differences in covariates between comparison groups as recorded; Weighted Sample, standardized differences in covariates between comparison groups after re-weighting of observations by inverse probability of being treated. 


\subsubsection{Treatment effects Average Treatment Effects}

Table 12 shows that the re-training programs had a significant influence on measured income and employment outcomes compared to the no re-training scenario. The estimated ATE of one-year re-training on nominal income was equal to $€ 22,839$, the ATE of two-year re-training was equal to $€ 35,620$. This was in comparison to the counterfactual scenario of no completed re-training. Without retraining, the average mean income over the eight-year observation period was assessed to be equal to $€ 81,961$. Including the effects of inflation, the ATE of one-year re-training on income was reduced to $€ 21,539$, while the ATE of two-year re-training on income was reduced to $€ 32,776$. All estimated differences in income were statistically significant at the one percent level.

An analysis of the number of days with employment showed that the income gains were mainly the result of more days in employment. The potential-outcome means with re-training were significantly larger compared to the potential outcome mean without re-training. Over the eight-year observation period, the average treatment effect of one-year re-training on the number of days in employment was equal to 322 days, while the ATE of two-year re-training was equal to 445 days. Both estimates were significant at the one percent level.

Additionally, there was a significant decrease in days with social security and unemployment benefits linked to the completion of both programs. The ATE of oneyear programs on the number of days with social security benefits was equal to -300 days, while the ATE of two-year programs was equal to -88 days. Both estimates were significant at the one percent level. Moreover, the ATE of re-training on the number of days with short-term unemployment benefits was equal to -50 days with a one-year re-training, and - 23 days with a two-year re-training. The estimated ATE of one-year re-training on short-term unemployment was significant at the one percent level. The effect of two-year re-training on the number of days with shortterm unemployment benefits was not statistically significant. On the other hand, the mean effect of one-year re-training on long-term unemployment was not statistically significant, whereas the ATE of two-year re-training on accumulative days with long-term unemployment benefits was significant at the one percent level. The ATE of one-year re-training on long term unemployment was equal to 64 days, while the ATE of two-year programs on the accumulative number of days with long-term unemployment benefits was equal to -142 days. 
Table 12: Potential outcome means and average treatment effects, one-year and two-year re-training in comparison to no re-training scenario

\begin{tabular}{|c|c|c|}
\hline & $\begin{array}{l}\text { Potential-Outcome } \\
\text { Mean (SE.) }\end{array}$ & $\begin{array}{l}\text { Average Treatment Effect } \\
(95 \% \text { CI. })\end{array}$ \\
\hline \multicolumn{3}{|c|}{ Income (in nominal $€$ ) } \\
\hline No re-training & $€ 81,961(€ 3,764)$ & \\
\hline 1yr re-training & $€ 104,801(€ 6,063)$ & $€ 22,839(€ 8,992, € 36,687)^{* *}$ \\
\hline $2 y r$ re-training & $€ 117,582(€ 1,910)$ & $€ 35,620(€ 27,546, € 43,695)^{* * *}$ \\
\hline \multicolumn{3}{|c|}{ Income (in $2005 €$ ) } \\
\hline No re-training & $€ 75,255(€ 3,424)$ & \\
\hline 1yr re-training & $€ 96,793(€ 5,710)$ & $€ 21,539(€ 8,549, € 34,528)^{* *}$ \\
\hline $2 y r$ re-training & $€ 108,030(€ 1,769)$ & $€ 32,776(€ 25,471, € 40,081)^{* * *}$ \\
\hline \multicolumn{3}{|c|}{ Employment (in days) } \\
\hline No re-training & $1,209(43)$ & \\
\hline 1yr re-training & $1,531(76)$ & $322(152,492)^{* * *}$ \\
\hline 2yr re-training & $1,654(20)$ & $445(354,536)^{* * *}$ \\
\hline \multicolumn{3}{|c|}{ Social security Benefits (in days) } \\
\hline No re-training & $677(23)$ & \\
\hline 1yr re-training & $377(25)$ & $-300(-366,-235)^{* * *}$ \\
\hline 2yr re-training & $589(7)$ & $-88(-136,-42)^{* * *}$ \\
\hline \multicolumn{3}{|c|}{ Short-Term Unemployment (in days) } \\
\hline No re-training & $185(11)$ & \\
\hline 1yr re-training & $136(12)$ & $-50(-82,-17)^{* *}$ \\
\hline $2 y r$ re-training & $162(5)$ & $-23(-47,1)$ \\
\hline \multicolumn{3}{|c|}{ Long-Term Unemployment (in days) } \\
\hline No re-training & $373(26)$ & \\
\hline 1yr re-training & $437(42)$ & $64(-33,160)$ \\
\hline 2yr re-training & $231(10)$ & $-142(-196,-88)^{* * *}$ \\
\hline \multicolumn{3}{|c|}{ Earnings Incapacity Pension (percentage) } \\
\hline No re-training & $10.1(1.5)$ & \\
\hline 1yr re-training & $9.1(1.8)$ & $-1.1(-5.6,3.5)$ \\
\hline $2 y r$ re-training & $4.3(0.5)$ & $-5.8(-8.9,-2.7)^{* * *}$ \\
\hline
\end{tabular}

Notes: Accumulative results after eight years. Long-term UE only from 2006-2010. Inverse Probability Weighted Regression Adjustment Method was used to estimate potentialoutcome means. The potential-outcome means refer to the average of the outcomes, for a specific level of re-training, given that all individuals would have attained this outcome. The average treatment effect measures the difference in these means. Final values were rounded to the nearest whole number (to the nearest tenth for percentage).

*** $\mathrm{P}<0.001{ }^{* *} \mathrm{P}<0.01{ }^{*} \mathrm{P}<0.05$ 
The re-training programs also influenced the uptake of a pension due to a reduced earnings capacity. While the likelihood to receive an earnings incapacity pension without re-training was equal to $10.1 \%$, it was $9.1 \%$ with one-year re-training and $4.3 \%$ with two-year re-training. The corresponding ATE of one-year re-training on earnings incapacity pension uptake of $-1.1 \%$ was not statistically significant. However, the ATE of two-year re-training on earnings incapacity pension uptake of $-5.8 \%$ was significant at the one percent level.

\section{Average Treatment Effects on the Treated}

Table 13 shows that the evaluated re-training programs had a large effect on the graduates' accumulative incomes and employment days. Without re-training, oneyear re-training graduates would have earned $€ 69,939$ over the eight-year observation period. With re-training, one-year program graduates, on average, had earned $€ 24,260$ more in comparison. Two-year program graduates, on average, would have earned $€ 84,445$ without the re-training measures, but were able to increase their income as a result of the re-training programs, on average, by $€ 35,972$. The corresponding ATET on real incomes were equal to $€ 22,742$ for one-year program graduates, and $€ 33,097$ for two-year program graduates. The income effects were statistically significant at the one percent level.

According to the analysis carried out, one-year program graduates were employed for 405 additional days in comparison to the counterfactual scenario without retraining. Two-year program graduates were employed for 441 additional days in comparison to the no-training scenario. The estimated ATET on employment days were significant at the one percent level. Moreover, one-year re-training graduates had 316 fewer days with social security benefits compared to the no re-training scenario. Two-year re-training graduates had 92 fewer days on social security benefits. Both estimated reductions were significant at the one percent level.

In addition, one-year program graduates, on average, had 26 fewer days on shortterm unemployment benefits in comparison to the non-training scenario, while twoyear graduates had 24 fewer days. However, differences in days with short-term unemployment benefits were not statistically significant for either group of retraining graduates. Moreover, in comparison to the potential outcome mean without re-training, one-year program graduates, had 20 additional days on long-term unemployment benefits, whereas two-year program graduates had 140 fewer days on long-term unemployment benefits. The estimated changes in long-term unemployment benefits were not statistically significant for one-year program graduates, but significant at the one percent level for two-year re-training graduates. Completing vocational re-training also had an effect on the likelihood of uptake of a pension due to a reduced earnings incapacity. The baseline likelihood of receiving 
an earnings incapacity pension without re-training was equal to $10.2 \%$. In comparison, it was 3.1\% lower for completers of one-year programs, and $6.0 \%$ lower for completers of two-year programs. The ATET was not statistically significant for one-year graduates, but significant at the one percent level for two-year graduates.

Table 13: Potential outcome means without re-training among treated individuals and average treatment effects on the treated, one-year and two-year re-training in comparison to no re-training scenario

\begin{tabular}{|c|c|c|}
\hline & $\begin{array}{l}\text { Potential-Outcome } \\
\text { Mean Without Re- } \\
\text { Training (SE.) }\end{array}$ & $\begin{array}{l}\text { Average Treatment Effect on } \\
\text { the Treated }(95 \% \text { CI.) }\end{array}$ \\
\hline \multicolumn{3}{|c|}{ Income (in nominal $€$ ) } \\
\hline 1yr re-training & $€ 69,939(€ 4,601)$ & $€ 24,260(€ 12,805, € 35,715)^{* * *}$ \\
\hline 2 yr re-training & $€ 84,445(€ 3,935)$ & $€ 35,972(€ 27,743, € 44,202)^{* * *}$ \\
\hline \multicolumn{3}{|c|}{ Income (in $2005 €$ ) } \\
\hline 1yr re-training & $€ 64,251(€ 4,250)$ & $€ 22,742(€ 12,150, € 33,334)^{* * *}$ \\
\hline $2 y r$ re-training & $€ 77,533(€ 3,637)$ & $€ 33,097(€ 25,495, € 40,700)^{* * *}$ \\
\hline \multicolumn{3}{|c|}{ Employment (in days) } \\
\hline 1yr re-training & $1,095(62)$ & $405(249,561)^{* * *}$ \\
\hline $2 y r$ re-training & $1,234(44)$ & $441(349,534)^{* * *}$ \\
\hline \multicolumn{3}{|c|}{ Social Security Benefits (in days) } \\
\hline 1yr re-training & $647(28)$ & $-316(-380,-251)^{* * *}$ \\
\hline 2yr re-training & $684(23)$ & $-92(-140,-45)^{* * *}$ \\
\hline \multicolumn{3}{|c|}{ Short-Term Unemployment (in days) } \\
\hline 1yr re-training & $170(17)$ & $-26(-66,13)$ \\
\hline $2 y r$ re-training & $188(11)$ & $-24(-48,0)$ \\
\hline \multicolumn{3}{|c|}{ Long-Term Unemployment (in days) } \\
\hline 1yr re-training & $451(43)$ & $20(-82,122)$ \\
\hline 2yr re-training & $357(26)$ & $-140(-194,-87)^{* * *}$ \\
\hline \multicolumn{3}{|c|}{ Earnings Incapacity Pension (percentage) } \\
\hline 1yr re-training & $10.2(1.9)$ & $-3.1(-7.8,1.6)$ \\
\hline 2yr re-training & $10.2(1.5)$ & $-6.0(-9.2,-2.9)^{* * *}$ \\
\hline
\end{tabular}

Notes: Accumulative results after eight years. Long-term UE only from 2006-2010. Inverse Probability Weighted Regression Adjustment Method was used to estimate potentialoutcome means. The potential-outcome means of no re-training refer to the average potential outcome, that would have occurred among those that graduated from a specific re-training had they not completed the re-training measures. The average treatment effect on the treated measures the difference in means with and without re-training for the subset of treated individuals. Final values were rounded to the nearest whole number (to the nearest tenth for percentage). ${ }^{* * *} \mathrm{P}<0.001{ }^{* *} \mathrm{P}<0.01{ }^{*} \mathrm{P}<0.05$ 


\section{Annual Income Development}

Figure 4 illustrates the annual, inflation-adjusted, income development of the retraining graduates in comparison to the no-training scenario. During the years 20072013, graduates of both re-training programs had significantly higher income compared to the counterfactual scenario of no-training. The positive effects of vocational re-training on income were sustained until the last year of the observation period. In the year 2013, the (adjusted) difference in real income of one-year program graduates was $€ 2,661,95 \%$ CI [€882, €4,439], higher compared to the non-training scenario. Two-year program graduates earned $€ 5,369,95 \%$ CI [€3,975, €6,764], more compared to the counterfactual scenario of no completed re-training.

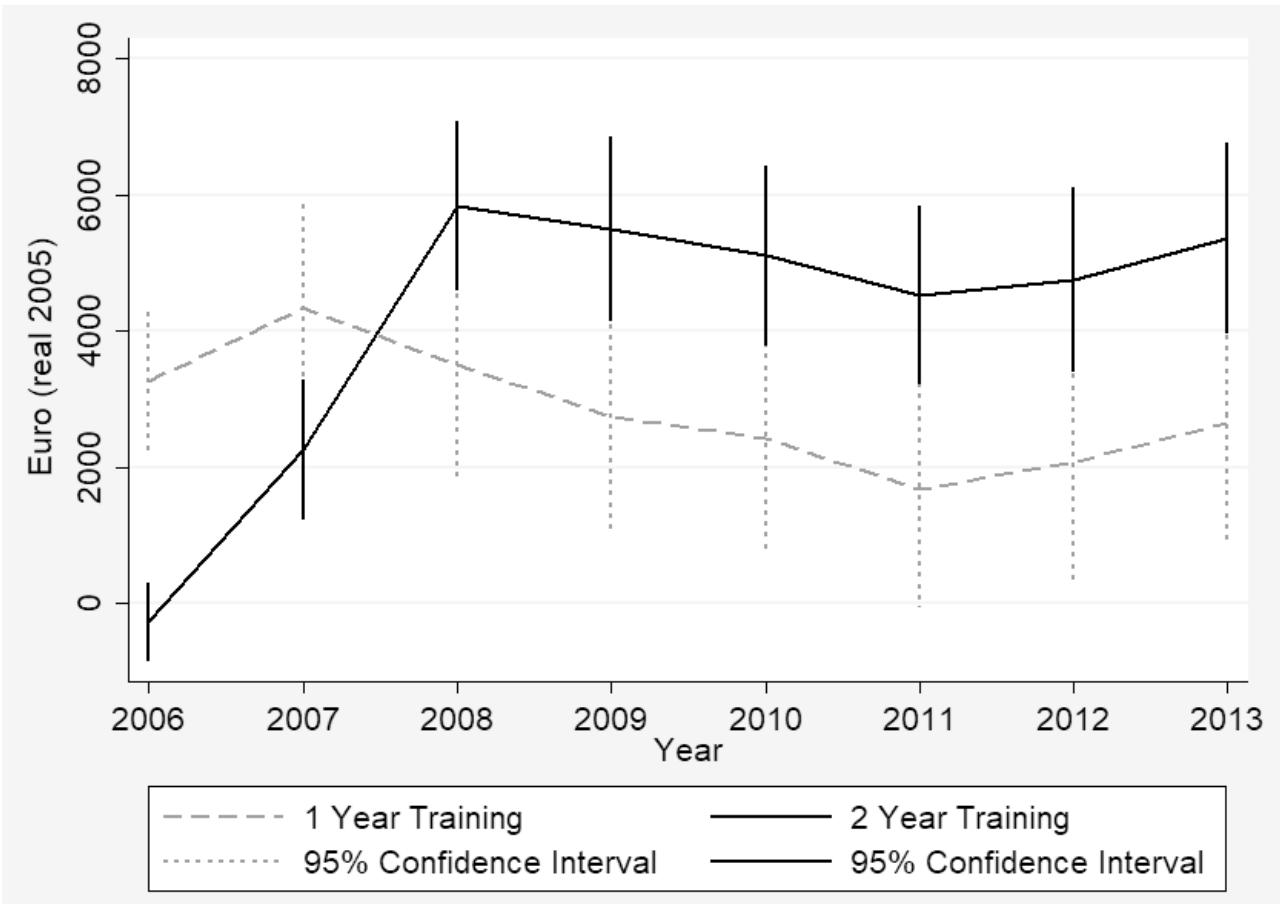

Figure 4 Graphical analysis real income development 2006-2013 in comparison to the scenario of no program completion; average treatment effect on the treated; annual estimates; vertical lines illustrate $\mathbf{9 5 \%}$ confidence interval.

Sensitivity Analysis: Only Non-Medical Program Dropouts

In a sensitivity analysis the control group was trimmed to participants who reported a non-medical reason for program non-completion. The most prevalent reasons for program non-completion were of a medical nature $(48.2 \%)$, followed by failed examinations $(21.8 \%)$ and other performance issues (6.5\%) (Table 9). It could be argued that participants who left the program for medical reasons carry some 
unmeasured confounders linked to their state of health, which negatively affect the employment situation. Thus, it was worth recalculating the model without program dropouts who left the program due to a reported medical reason.

Leaving out data from 177 participants reduced the comparison group to 190 observations (overall $\mathrm{n}=2222$ ). The analysis of standardized differences suggested that the distribution of covariates between comparison groups were balanced after observations were re-weighted by the inverse probability of being treated. All differences in covariates were reduced to a negligible level, the necessary condition to carry out the impact analysis. According to the reduced sample analysis, the adjusted differences in income were still significant but slightly reduced in absolute value in comparison to the treatment effects on the full sample. Over the eight-year observation period, the nominal treatment effect on the treated for one-year program graduates was reduced to $€ 22,451,95 \%$ CI $[€ 8,238, € 36,664]$, and for two-year program graduates to $€ 32,246,95 \%$ CI [ $€ 21,568$, $€ 42,923]$. For the other outcome measures, the estimates were trimmed by a similar margin.

\subsection{Discussion}

In this chapter, an empirical example of how to use administrative panel data when evaluating re-training programs for people with disabilities has been provided. The main research goals of this analysis were linked to finding a viable control group and linking various data sources to examine the long-term effectiveness of vocational re-training measures for a representative group of rehabilitants in Germany. Those effects include changes in individual earnings and days with employment, earnings incapacity pension receipt and changes in the number of days on social security and unemployment benefits. Based on a random sample of 2,399 individuals admitted into vocational re-training, the effectiveness of the measures was examined by estimating the average treatment effect and the average treatment effect on the treated in relation to the case of no completed re-training.

The choice of method for the analysis of absolute treatment effects in this study adds to the methodological debate on how to estimate program effects from observational data for public policymaking. The main point of debate is whether policymakers can confidently rely on treatment effect estimates obtained from quasi-experimental research settings. Much of the literature indicates that the most common weakness in the quasi-experimental research setting is linked to using an external comparison group. While different training programs exhibit different institutional settings, the foregoing analysis suggests that it is possible, at least for the case of vocational retraining in Germany, to make use of information on program dropouts as an appropriate internal control group to obtain meaningful treatment effect estimates 
While this study only included a relatively small number of controls in relation to the number of treated subjects, the analysis of standardized differences has shown that despite the relatively small number of controls, effective re-weighting was carried out, which limited the differences in covariates between comparison groups to a negligible level. This was the prerequisite to carry out the impact analysis.

The results of this analysis suggest that both types of vocational re-training programs analyzed significantly improved the income and employment situation of the individuals involved over the first eight years after program admission. After other factors had been controlled for, graduates of one-year re-training were on average employed for an additional 405 days, 95\% CI [249 days, 561 days], and had earned $€ 24,260$ more than without re-training, 95\% CI [€12,805, €35,715]. Two-year program completers, on average, were employed for 441 additional days, $95 \% \mathrm{CI}$ [349 days, 534 days], and had earned $€ 35,972$ more than without re-training, 95\% CI $[€ 27,743, € 44,202]$. Moreover, completing a re-training program significantly reduced the number of days on social security benefits and influenced the number of days on unemployment benefits. In addition, the measures also reduced the likelihood of receiving an earnings incapacity pension in comparison to the counterfactual scenario of no re-training. Over the eight-year observation period, one-year program graduates had a 3.1\% lower likelihood of being awarded a pension due to a reduced earnings capacity, 95\% CI [-7.8\%, 1.6\%]; two-year program graduates had a $6.0 \%$ lower likelihood, 95\% CI [-9.2\%, $-2.9 \%]$.

When drawing causal conclusions from the estimated relationships the set-up of the quasi-experiment should always be kept in mind. The association between the duration or level of schooling and earnings does not necessarily imply causality. Program dropouts participated in the re-training measures before leaving the program, thus, also profiting from the skill training, while at the same time, using up time that could have been spent otherwise if they would have never participated in the measures at all (e.g. to earn money or to get a different training). On the one hand, failed examination and other performance issues being common among the group of program dropouts' signal that the former might have carried some unmeasured confounders, which could negatively affect their earnings performance. This would mean that the estimated causal relationships in this analysis were upward biased, due to the negligence of these factors. On the other hand, the argument can be brought forward, that many of the program dropouts likely discontinued their training because they found another job opportunity (perhaps even linked to the benefits received from participating in re-training). This would mean that the treatment effects were underestimated, since some of the wage 
improvement in the control group was due to knowledge and skills attained during the period of rehabilitation.

Regarding the relative effectiveness of the two interventions studied, the estimated mean employment effects were generally larger for two-year re-training programs in comparison to one-year programs (despite longer program duration), indicating that two-year programs have a larger impact. However, the response to treatment is likely to be heterogeneous and dependent on the individual situation and preferences of the rehabilitants. Program participation depends both on rehabilitant eligibility and on the selection by the person in charge or self-selection by potential participants.

To better understand the individual needs and responses to training, it would be relevant to further explore the perspective of the individual before and during the period of rehabilitation as well as the role of the provider in programmatic decision making. While about half of program dropouts left the program for medical reasons, further explorative analysis is also needed to improve the assistance provided during the period of rehabilitation to prevent the occurrence of medically related dropouts. This includes knowing more about the barriers with regards to the access to the re-training measure, which would allow a more accurate prediction of when re-training is medically appropriate and what type of additional assistance (accommodation) is needed for the measures to have the greatest chance of success.

In addition, further analysis could investigate whether program dropout could have been predicted before the start of the measures based on better screening and program allocation mechanisms. Apart from medical reasons for program dropout, failed examinations and other performance issues were the other main reasons why individuals were not able to finish a re-training program. Consequently, future research should further investigate possible assistance that can be provided during rehabilitation to increase graduation rates. Moreover, the outcome of allocation practices should be further examined: Were the individuals satisfied with the occupational re-training choice? Would they rather have enrolled in a different trade or occupation? Were there other reasons that influence how well the rehabilitants perform in the examinations? Given the large benefits associated with completed retraining and the comparatively large costs of these measures, these are relevant questions.

A potential drawback of this study is that the range of potential variables for calculating the propensity score was limited to a fixed number of registered covariates. Although this study incorporates various proxies to model the earnings ability of the rehabilitants, it was not possible to observe or measure the 
rehabilitants' motivation or other soft factors that have an influence on employment outcomes, which may lead to imprecise estimates. Incorporating proxies for these "factors" into future analysis could contribute to a better understanding of individual education and work reintegration trajectories. Another interesting area for future research could be to address potential measurement error in the treatment variable using instrumental variables methods. In the absence of randomized control trials, policy changes could be a source of promising instruments since they affect the allocation to treatment independently of preferences. Other, possible instruments include the distance to the nearest training facilities or the parental education level. ${ }^{15}$

Despite these potential limitations, the treatment effects presented in this study are an important contribution to the empirical knowledge on the influence vocational re-training on the employment re-integration of individuals with disabilities in Germany. This claim is based on the availability of a unique database and the application of appropriate statistical methods, which have allowed a thorough evaluation of the impact of the re-training programs. According to the estimated treatment effects, completing a vocational re-training was associated with significant improvements in the employment status while also reducing dependency on social security benefits. Policies to promote the labor market reintegration of persons with disabilities should consider that vocational re-training may be an effective tool for sustainably improving work participation outcomes.

\footnotetext{
15 The distance to a training facility is generally considered to be one of the most important non-monetary barriers impeding access to education programs. On the other hand, parental education instruments build on the concept of inter-generational correlation of education outcomes.
} 


\subsection{Appendix to Chapter 3}

The counterfactual Framework in the analysis of treatment alternatives

Using the terminology of Wooldrige, Jeffrey, (2011), the impact of a treatment on individual $i, \delta i$, is the difference between potential outcomes with and without treatment:

$$
\delta_{i}=Y_{i 1}-Y_{i 0}
$$

To estimate the impact of a program over the population, several possibilities have been suggested in the literature. Since the effect to be measured, is an individual specific, random variable, it has to be specified which feature of the distribution is to be estimated (Wooldridge, Jeffrey, 2010). Rosenbaum \& Rubin (2006) suggest estimating the average treatment effect (ATE), which is the expected effect of treatment on a randomly drawn person from the population (Wooldridge, Jeffrey, 2010, p. 905f):

$$
\tau_{\text {ate }}=E\left(y_{1}-y_{0}\right)
$$

It can be argued that, for policy purposes, this is not the best quantity of interest since it averages across the entire population, thus, potentially including subjects into the estimate of the average who would, in fact, never be eligible for treatment (Wooldridge, Jeffrey, 2010). A better-suited quantity for policy purposes might, therefore, be the average treatment effect on the treated (ATET). The ATET is the mean effect for those who actually participated in the program:

$$
\tau_{\text {atet }}=E\left(y_{1}-y_{0} \mid w=1\right)
$$

Where $\mathrm{w}=1$ refers to the treatment and zero otherwise. The second term is the average outcome of treated individuals had they not received the longer treatment. The difficulty in estimating either the ATE or ATET is that only $y_{0}$ or $y_{1}$ is observed for a single individual, never both. In fact, along with the treatment variable $\mathrm{w}$, the observed outcome is:

$$
y=(1-w) y_{0}+w y_{1}=y_{0}+w\left(y_{1}-y_{0}\right)
$$

If treatment assignment was purely random such that the treatment indicator w was statistically independent of $y_{0}$ and $y_{1}$, the estimation of treatment effects would be straight forward since ATET and ATE would converge to the same value:

$$
\tau_{\text {ate }}=\tau_{\text {atet }}=E(y \mid w=1)-E(y \mid w=0)
$$

However, pure randomization is often unfeasible since, for example, people deemed to be participating in rehabilitation measures usually cannot be denied access to the 
services. Moreover, the individuals themselves, as well as the agency in charge of program allocation, at least partly determine whether they receive treatment and what type. Whereas their decisions may be related to the anticipated benefits from training, placement mechanisms can be thought of being non-random (Wooldridge, Jeffrey, 2010).

\section{Assumptions}

The availability of relevant data on pre-treatment outcomes and patient characteristics, however, allow ATE and ATET to be estimated under less restrictive assumptions than independence between $\left(y_{0}, y_{1}, w\right)$ (Wooldridge, Jeffrey, 2010). Rosenbaum \& Rubin (2006) developed the "ignorability of treatment" assumption, sometimes also called un-confoundedness or conditional independence assumption. Under the ignorability of treatment assumption, conditional on a set of measured covariates $(\mathrm{X})$, the assignment of units to treatment is 'as good as random' (Rosenbaum \& Rubin, 2006):

Conditional Independence: $\quad\left(y_{0}, y_{1}\right) \perp \mathrm{w} \mid \mathrm{X}$

In Heckman \& Robb (1985) this strategy is also referred to as the "selection on observables strategy". It requires that all variables thought to be influencing the outcomes are observed and included in the group of covariates. It provided the basis for using the results of the untreated subject to construct an unbiased counterfactual for the treatment group. Naturally, the conditional independence assumption seems to have a better chance of holding with a richer set of control variables. For the results to have a meaningful interpretation, common support (overlap in measured covariates) is needed to evaluate treatment effects (Wooldridge, Jeffrey, 2010). Common support refers to the property that, for all possible vectors of $\mathrm{X}$, there is a positive probability of being both treated and untreated:

Common support: $\quad 0<P(w=1 \mid x)<1$

When both properties, the conditional independence and the common support, are satisfied, the treatment assignment is said to be strongly ignorable using the original terminology of Rosenbaum \& Rubin (2006), and ATE and ATET can generally be specified (Wooldridge, Jeffrey, 2010). The main challenge is similarity; the goal is to find training participants who can be thought of being very similar in terms of observable characteristics that affect employment outcomes.

\section{The propensity score model}

The most frequently used balancing score is the propensity score; the probability to be treated given the observed characteristics. This has two reasons: First, adjusting for differences in the propensity score between treated and control groups 
eliminates all systematic biases associated with differences in observed patient characteristics. Second, using propensity score methods the "similarity" aspect of the estimation is reduced to a single dimension; naturally, it is easier, to find close matches on a scalar function of the observed characteristics, compared to finding close matches on all covariates observed (G. W. Imbens \& Rubin, 2015).

Formally, let $X i$ denote the individual vector of pre-treatment characteristics, while the treatment status is reflected by $w_{i}$. Then the conditional probability of treatment, the propensity score, can be denoted as:

$$
\operatorname{Pr}\left(w_{i}=1 \mid x_{i}, e\left(x_{i}\right)\right)=\operatorname{Pr}\left(w_{i}=1 \mid x_{i}\right)=e\left(x_{i}\right)
$$

The assumption that motivates the covariate adjustment is that conditioning on $\mathrm{Xi}$ eliminates selection bias. If treatment assignment is strongly ignorable given $X$, (that is $\left.\left(y_{0}, y_{1}\right) \perp \mathrm{w} \mid x\right)$, then it is strongly ignorable given any balancing score, i.e. the propensity score. Accordingly, at any value of a balancing score, the difference between the treatment and control means is an unbiased estimate of the average treatment effect at that value of the balancing score (Rosenbaum \& Rubin, 2006).

The motivation for focusing on propensity score methods is linked to the high dimensionality of the observable characteristics that have an effect on studied outcomes (Dehejia \& Wahba, 2002). Because there are usually various explanatory variables available, it is cumbersome to determine along which dimensions to compare subjects and which weighting scheme to adopt. In those circumstances, propensity score methods can provide a natural weighting scheme that yields unbiased estimates of the treatment impact (Dehejia \& Wahba, 2002). The value of propensity-score matching is in the dimension reduction generated by regions in the support of $\mathrm{X}$ where $\mathrm{p}(\mathrm{Xi})$ is constant but $E\left(y_{i 1} \mid x_{i}\right)$ and $E\left(y_{i 0} \mid x_{i}\right)$ are not (Angrist \& Hahn, 2004).

Usually a probit or logit model is used to estimate conditional treatment probabilities. The probit model employs a probit link function ${ }^{16}$ and is estimated using the maximum likelihood procedure. Probit models were first introduced in the 1930s (Bliss, 1934). Probit models are similar to logit models in that they are specifications for modeling binary response to treatment given a range of observed independent variables. Our analysis makes use of Imbens' generalization of the propensity score (G. W. Imbens, 2000), which showed that the results of Rosenbaum

\footnotetext{
16 The probit function is the quantile function associated with the standard normal distribution. Mathematically, it is defined as the inverse of the cumulative distribution function of the standard normal distribution.
} 
\& Rubin (Rosenbaum \& Rubin, 2006) continue to hold when the treatment is multivalued. Accordingly, a multinomial logit model was used to calculate the conditional treatment probabilities.

\section{Model selection}

When deciding on variables to be included in the mode, it must be ensured that no variables are included that themselves are affected by treatment. For example, including a measure of education obtained after program assignment would hold additional education fixed when it actually varies in response to treatment assignment (Wooldridge, Jeffrey, 2010). Accordingly, proper candidates for being included in the set of covariates are variables measured prior to treatment assignment. This includes past outcomes of the dependent variable (Wooldridge, Jeffrey, 2010). It is suggested to use a flexible probit model in which various levels of the independent variables, for example, level, squares, and interactions are included (Dolton \& Smith, 2011; Wooldridge, Jeffrey, 2010).

As Bitton, Onega, Tosteson, \& Haas (2014) note, selection models should include all variables thought to influence the outcome, even if they do not influence the treatment decision. Specifically, in this case, the model should only include variables that correlate with labor market earnings. On the other hand, instruments, which are variables that influence solely the treatment decision but not outcomes, should not be included in standard treatment selection models since they bias results (Wooldridge, Jeffrey, 2010). Rosenbaum, Paul R, Rubin (1984) present an iterative approach to specifying a propensity score model. The first step is to manually specify an initial propensity score model with only a few variables. Next, further relevant variables are added, and the comparability of treated and untreated subjects in the matched samples and the size of the coefficient in the outcome models were assessed. If there are important systematic differences in covariates between the two groups or potential omitted variables, the model should further be modified. This modifying can be done by adding additional covariates, nonlinear terms and interaction terms (Austin, 2011).

With the assistance of statistical software, this process can be sped up significantly. Using Statas user-written "bfit" command, combinations of explanatory variables can be tested to identify models that exhibit high efficiency based on the combination of covariates included. ${ }^{17}$ The statistical criteria tested to establish efficiency are the Bayesian Information Criterion (BIC) and the Akaike Information Criterion (AIC). Both, the BIC and the AIC, are penalized-likelihood information

\footnotetext{
17 MacDonald et al. (2004) introduce this technique.
} 
criteria that enable cross-comparison of different models using statistical power analysis (Dziak et al., 2012).

The two information criteria support different models depending on the trade-off between the relative importance assigned to specificity versus sensitivity. Specificity refers to not overfitting a model or suggesting non-existent relationships while sensitivity refers to having sufficient parameters to model the relationships among variables (Dziak et al., 2012). Under the BIC, the optimal model exhibits fewer terms compared to the AIC due to the penalty given to higher degrees of freedom. In contrast, under the AIC, the optimal model includes more terms being more optimistic and the resulting penalty for model complexity being comparatively weak.

Table 14 shows the different variables included in the models with the lowest BIC and AIC, respectively. In accordance with the theory, the BIC model had a higher joint significance, an F-statistic of 44.36 vs. 28.91, whereas the model with the lowest AIC was able to explain more of the variation in the dependent variable (Adj. Rsquared $=0.2391$ vs. 0.2244 ). The specification used in our final model included all variables suggested by the BIC. Additionally, three further variables were added to the model. These were the interaction term between before application earnings (2004) and age at admission, the education dummies and the ICD dummies. Three other terms included in the model suggested with the lowest AIC score (Wage2004², Age $^{2}$ and Wage2003*Age), were not used in this final specification because they correlate strongly with already included variables while not being existential for the conditional independence assumption to hold.

The final specification of the propensity score model to estimate the conditional treatment graduation probabilities had the following functional form:

$$
\begin{aligned}
\operatorname{Mlogit}(w)=\beta_{0} & +\beta_{1} * \text { Wage } 2003^{2}+\beta_{2} * \text { Wage } 2003+\beta_{3} * \text { Wage } 2004+\beta_{4} * \text { Age } \\
& +\beta_{5} * \text { Wage } 2004 * \text { Age }+\beta_{6} * \text { Working Status }+\beta_{7,8,9} \ldots 15 \\
& * \text { Job Type }+\beta_{16} * \text { Sex }+\beta_{17-20} * \text { Education }+\beta_{21,22} \\
& * \text { ICD diagnosis }+\beta_{23} * \text { Region }+e
\end{aligned}
$$

Some of the variables in the final model were not individually statistically significant but the likelihood ratio test statistic for the model was large (Prob $>$ chi2 $=0.0000$ ). The F-statistic and adjusted variation explained of the final model were somewhere in between the models with the optimal BIC and AIC (F-statistic = 33.80; Adjusted rsquared $=0.2366$ ). 
Table 14: Comparison of independent variables included in the optimal model under the Bayesian and the Akaike Information Criterion and final model used

\begin{tabular}{lccc}
\hline & $\begin{array}{c}\text { Optimal } \\
\text { BIC }\end{array}$ & $\begin{array}{c}\text { Optimal } \\
\text { AIC }\end{array}$ & $\begin{array}{c}\text { Final } \\
\text { Model }\end{array}$ \\
\hline Prior work Experience & & & \\
Wage20032(cont.) & - & $\mathrm{O}$ & $\mathrm{O}$ \\
Wage2003 (cont.) & $\mathrm{O}$ & $\mathrm{O}$ & $\mathrm{O}$ \\
Wage2004 ${ }^{2}$ (cont.) & - & $\mathrm{O}$ & - \\
Wage2004 (cont.) & $\mathrm{O}$ & $\mathrm{O}$ & $\mathrm{O}$ \\
Wage2003 * Age (cont.) & - & $\mathrm{O}$ & - \\
Wage2004* Age (cont.) & - & $\mathrm{O}$ & $\mathrm{O}$ \\
Working status (1 dummy) & $\mathrm{O}$ & $\mathrm{O}$ & $\mathrm{O}$ \\
Job type dummies (9 dummies) & $\mathrm{O}$ & $\mathrm{O}$ & $\mathrm{O}$ \\
Demographic Factors & & & \\
Age ${ }^{2}$ (cont.) & - & $\mathrm{O}$ & - \\
Age (cont.) & $\mathrm{O}$ & $\mathrm{O}$ & $\mathrm{O}$ \\
Sex (1 dummy) & $\mathrm{O}$ & $\mathrm{O}$ & $\mathrm{O}$ \\
Education (4 dummies) & - & $\mathrm{O}$ & $\mathrm{O}$ \\
ICD (2 dummies) & - & $\mathrm{O}$ & $\mathrm{O}$ \\
Residential Region (1 dummy) & $\mathrm{O}$ & $\mathrm{O}$ & $\mathrm{O}$ \\
\hline Constant & $\mathrm{O}$ & $\mathrm{O}$ & $\mathrm{O}$ \\
\hline F & 44.36 & 28.91 & 33.80 \\
Prob > F & 0.00 & 0.00 & 0.00 \\
Adj. R-squared & 0.2244 & 0.2391 & 0.2366 \\
$\mathrm{~N}$ & 2,399 & 2,399 & 2,399 \\
\hline Not Dep
\end{tabular}

Notes: Dependent Variable: Income 2006-2013. $\mathrm{O}=$ variable was included in the model. The maximum order of the covariate polynomials tested in this analysis was a fully interacted second-order polynomial.

\section{Estimation}

In this study, the inverse probability weighted regression adjustment (IPWRA) method was used to estimate treatment effects. The IPWRA estimator used in this study is part of the class of "Wooldridge's double-robust" estimators, derived in Wooldridge (2007) and discussed at length in (Wooldridge, Jeffrey, 2010). The method is characterized by a multi-equation model consisting of an equation for the program selection (in this case a multinomial logit model), an outcome equation for the treated state and an outcome equation for the other treatment and non-treated state. 
The propensity score was estimated using multinomial logistic regression in order to establish comparability, modeling the program completion status as the dependent variable and individual characteristics as independent variables. The analysis made use of Imbens' generalization of the propensity score (G. W. Imbens, 2000), which showed that the results of Rosenbaum \& Rubin (Rosenbaum \& Rubin, 2006) continue to hold when the treatment is multi-valued. ${ }^{18}$ Using Statas' built-in "mlogit" command, conditional treatment probabilities were estimated. The "mlogit" command fits maximum-likelihood multinomial logit models, also known as polytomous logistic regression. ${ }^{19}$

Next, regression models of the employment and social security outcomes were fitted for each treatment level separately (OLS for continuous outcomes, Probit for discrete). In the regression models, observations were weighted by the inverse probability of treatment (i.e. the conditional probability of people completing a program). The results of the models were used to predict treatment-specific potential outcomes for each subject in both the treated and the non-treated state (of which only one is observed in reality).

The means of the treatment-specific outcomes were computed among all individuals in the sample. The differences in these averages provide the estimates of the ATE. In addition, because researchers and policymakers might be more interested in the causal treatment effects only for those who successfully completed the re-training measures, the ATET was calculated. The ATET was calculated by limiting the observations to the subset of two-year (one-year) graduates before estimating the mean differences between the outcomes with and without program completion.

Methods based on the inverse probability weight have several advantages over traditional matching techniques: First for IPW methods, data is simply reweighted using the estimated propensity score, thus, not requiring the choice of a caliper width. Second, under certain assumptions, the inverse probability method achieves the semi-parametric efficiency bound (Hirano, Imbens, \& Ridder, 2003). Third, the approach exhibits the double robust property meaning that it applies the selection and outcome model simultaneously producing a consistent estimate of the parameter if either of the two models is correctly specified (Wooldridge, Jeffrey, 2010). A simple proof that the doubly robustness property holds for certain combinations of conditional mean specifications is provided in Wooldridge (2007).

\footnotetext{
${ }^{18}$ See also the papers by Imbens (Imbens, GW, 2004) for further explanations.

${ }^{19}$ For an introduction to multinomial logit models, please see Greene, 2012; Hosmer, Lemeshow, \& Sturdivant, 2013; Long \& Freese, 2014.
} 
4. One- and two-year re-training in comparison: does the extra year pay off? 


\subsection{Introduction}

The previous chapter showed how completing either type of vocational re-training program (partial or full) was linked to improvements in the employment situation and a reduced dependency on social security benefits, when compared to the alternative of no re-training. In this chapter, the analysis is extended to examine the extent to which one-year and two-year vocational re-training programs have similar effects on employment outcomes. Although the shorter re-training programs require less time and smaller financial resources, recent assessments have found that they might provide employment effects comparable to those of the traditional two-year programs.

Traditionally, the focus of German vocational rehabilitation programs has been on long-term re-training measures that are often completed by formal examinations. In the light of political initiatives to strengthen horizontal training (qualification for additional tasks), partial qualifications are becoming increasingly popular as an alternative to the more involved full re-training programs. Following criticism regarding long-lasting lock-in effects of the traditional two-year programs, there has been an uprising of partial re-training measures that usually can be completed within a year.

A recent evaluation of the differential impact of partial and full re-training measures (Bethge \& Streibelt, 2015) suggests that the one-year programs provide similar employment effects to those of the traditional two-year program. Using four years of follow up data, the authors show that, for the matched rehabilitants, partial qualifications led to integration results comparable to those of the longer two-year programs. Their results are linked to the earlier findings by Biewen \& Waller (2007), who identified that short-term programs are effective when compared to the traditional and more expensive longer-term degree programs. A similar finding can be found in Fitzenberger \& Völter (2007), who compared the effectiveness of publicsponsored training programs with those of the German federal employment office.

However, the evaluation periods in the previously published reports are relatively short compared to the duration of the measures studied. According to Streibelt \& Egner (2012), the time of follow up strongly influences studied outcomes. As such, there is a demand for empirical evidence on long-term outcomes to assess the question of long-term effectiveness and efficiency. Education researchers agree that the impact of an educational program may not be measurable for several years after the program has taken place. This delayed impact can be caused by a variety of factors, such as program lock-in effects, transitional periods after graduation with interrupted employment contracts, unavailability to the labor market (e.g. due to 
enrolling in another education or training program) or (lack of) seniority, all of which may reduce short-term graduate productivity.

To determine whether program allocation into a one- or two-year program is, on average, more effective, the employment outcomes of the two programs were directly compared. This comparison was performed on two levels: first, average treatment effects were calculated to compare the potential outcomes of all rehabilitants under each treatment scenario. Second, the analysis of the treatment effect was limited to the subset of individuals who participated in a two-year retraining. This was done in order to evaluate the extent to which the additional year of education had an influence on the level of income earned and on social security benefit dependency among those that actually participated in the longer re-training programs. In addition, the potential outcome means with two-year re-training among treated individuals with a one-year re-training were estimated in order to assess the potential effect of the longer re-training for those that only received the shorter-duration re-training. In the analyses, a "selection on observed variables strategy" was adopted, making use of available pre-treatment data on the rehabilitants' socio-economic status to identify conditional treatment probabilities. The doubly robust inverse probability weighted regression adjustment method was used to estimate treatment effects for the comparison groups.

The remainder of this chapter is structured as follows: The next section presents the methods, with a description of the study design, the study population, the retraining programs and the data used. Moreover, the statistical analysis method is summarized. In the results section, descriptive statistics of the participants are presented and the balance in covariates between comparison groups is evaluated before relative treatment effects of the re-training measures are presented. In the final section of this article, shortcomings of the findings are discussed before conclusions from the research are offered.

\subsection{Methods}

\subsubsection{Study design and population}

To investigate the relative employment effects associated with two- or one-year vocational re-training in Germany, a retro-perspective, quasi-experimental, cohort study was performed. Often valid control groups can be found by analyzing people in different training programs because the best alternative to a certain educational program is not necessarily to receive no education but to receive a different treatment type instead. In this research, the incremental impact of two-year retraining measures was assessed by estimating average treatment effects in comparison to hypothetical allocation into a one-year program. In the absence of 
randomization, a propensity score-based method was used to establish comparability between comparison group.

The data used for this analysis was collected from the administrative data records of the German Statutory Pension Insurance Fund (Deutsche Rentenversicherung Bund). It comprised accurate, process-generated information on the earnings development and insurance relationship for a cohort of rehabilitants in the eight years after admission into re-training and two years prior. The study population consisted of people with disabilities who, due to their health problem, were no longer able to (or would, in the foreseeable future, no longer be able to) carry out prior job tasks. Additionally, they must have successfully applied for vocational rehabilitation with the German Statutory Pension Insurance Fund ${ }^{20}$.

Once the application for rehabilitation had been approved, suitable re-training programs were chosen from a large pool of certified private or public institutions specialized in different professions and skill training. Vocational education in Germany can generally be categorized into partial and full vocational re-training programs. Apart from the fact that both types of re-training programs require fulltime participation, they differ considerably in content and length.

Full re-training programs are comparable to regular apprenticeships programs, typically lasting for two years. During the programs, classroom training is combined with on-the-job training to learn skills and obtain a professional qualification in a new field of work. A formal examination is often set at the end of the programs. Partial qualification measures, on the other hand, aim at extending existing competencies with additional skills, e.g. in the fields of business administration or information technology. The measures are usually completed within a year, aiming to reintegrate the participants more quickly into working life.

\subsubsection{Data}

The data used for this analysis was retrieved from administrative records made available by the Research Data Centre of the German Statutory Pension Insurance Fund. The corresponding data is representative of the whole rehabilitant population and featured accurate process-generated information on the income and employment development before and after participation in a rehabilitation measure.

The retrieved data consisted of three databases: The first database included a random sample of all recognized vocational education measures in 2005 and a range

\footnotetext{
${ }^{20}$ The different eligibility and exclusion criteria for vocational rehabilitation have already been presented in Section 3.2.1 and are, therefore, not repeated here.
} 
of variables linked to implementing the rehabilitation programs. The second database was retrieved from the pension insurance follow-up database in order to include information on the insurance relationship of the participants for two years before the program started and eight years after (2003-2013). To add further information on the rehabilitants' backgrounds, a third database was added to control for fixed individual characteristics. Lastly, a fourth dataset provided information on whether a pension due to a reduced earnings capacity was paid out as well as the level of the pension benefits. This information was also collected directly by the Research Data Centre.

\subsubsection{Comparison groups}

In order to compare the effects of full and partial re-training programs, the data was stratified according to allocation into either the two-year or one-year re-training (horizontal comparison Figure 5). This was achieved by creating a bivariate indicator variable, which reflected whether the rehabilitants participated in a one-year retraining (value $=0$ ) or participated in a two-year re-training (value $=1$ ). ${ }^{21}$ In total, the group of one-year participants included 317 observations, while the group of twoyear participants included 2,082 observations.

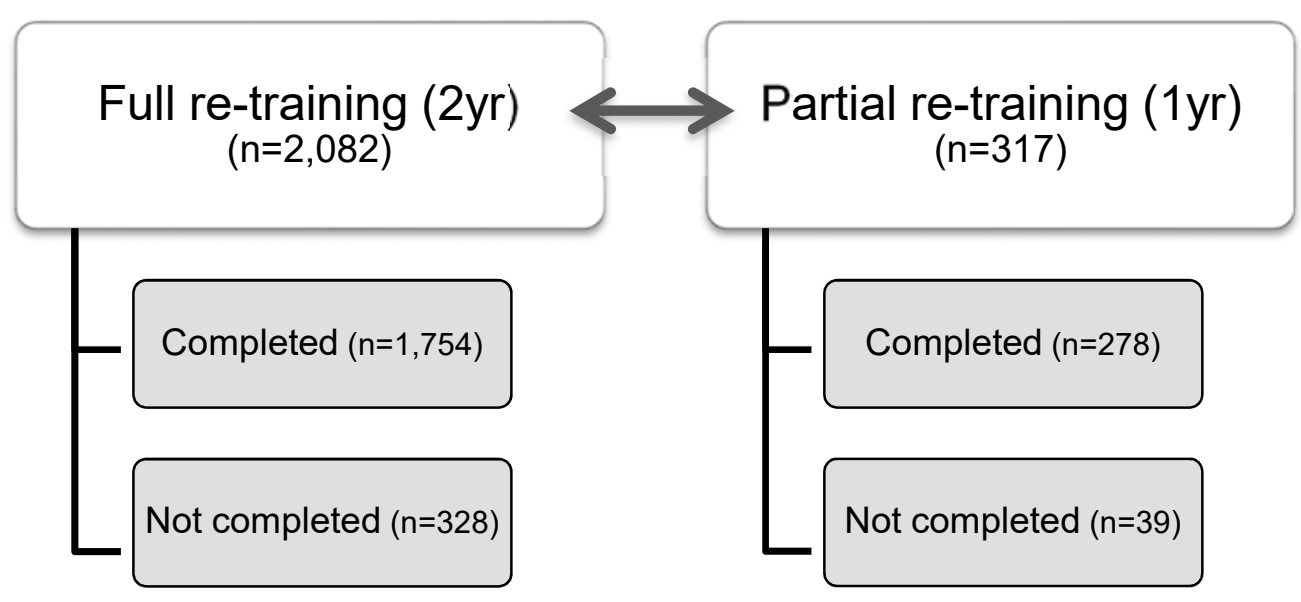

Figure 5 Comparison groups: duration effects of vocational re-training

${ }^{21}$ The outcome of the vocational re-training measure was identified using the variable "BFEWMS" from the provided dataset. 


\subsubsection{Outcome measures}

Participation in paid employment was assessed using the nominal and real income, as well as employment days, in the first eight years after program admission (20062013). This data was collected directly from the provided pension insurance followup database. A potential drawback of the data used was that employment data collected by the German pension insurance is limited to a contribution ceiling. For annual incomes above $€ 69,600$ ( $€ 58,800$ in the former East German states), the median value of $€ 77,179$ ( $€ 65,400)$ was recorded in the data instead of the true value. This potentially lowers the estimated program impact, as annual income above the specified cut-off value were not properly recorded. Changes in real income were estimated using the average of historical inflation rates collected from the German Federal Bureau of Statistics between 2003 and 2013 (Destatis, 2018). The mean annual discount factor used was equal to $1.6 \%$.

Secondary outcomes evaluated were days on social-security benefits, days on shortterm unemployment benefits, days on long-term unemployment benefits and an indicator variable signaling whether a pension due to a reduced earnings capacity was awarded. The data on social-security benefits included sick pay and temporary allowance payments made during the period of rehabilitation. Short-term unemployment benefits are benefits of the German unemployment insurance, which are paid on the occurrence of unemployment. They are usually paid for up to one year, and for older unemployed people, for up to two years. Long-term unemployment benefits are the basic (means-tested) social security benefits for employable persons in Germany, who are unemployed for longer than 12 months. Data on days with long-term unemployment benefits was only available from 20062009. Workers who are only able to work a few hours a day because of their health can apply for a reduced earnings capacity pension. An individual can claim a reduced earnings capacity pension, if for the foreseeable future, because of ill health or disability, they are unable to do more than three hours of paid work a day.

\subsubsection{Covariates}

The following variables from the demographic database were included as independent variables in the calculations: age, gender and level of education. Additionally, the residential region, the main medical diagnosis and the employment status were retrieved from data collected during the application process. Moreover, based on the information provided from the insurance followup database, measures of past earnings performance were included: the nominal income earned in the year 2003 and 2004 and the last registered occupation type. 


\subsubsection{Econometric approach}

To determine whether program allocation into a one- or two-year program was, on average, more effective, the employment outcomes of the two programs were directly compared. In the analysis, a "selection on observed variables strategy" was adopted, making use of available pre-treatment data on the rehabilitants' socioeconomic statuses to identify conditional treatment probabilities. A probit model was used to model program allocation as a function of the measured covariates:

$$
\begin{aligned}
\operatorname{Probit}(w)=\beta_{0} & +\beta_{1} * \text { Wage } 2003^{2}+\beta_{2} * \text { Wage } 2003+\beta_{3} * \text { Wage } 2004+\beta_{4} * \text { Age } \\
& +\beta_{5} * \text { Wage } 2004 * \text { Age }+\beta_{6} * \text { Working Status }+\beta_{7,8,9} \ldots 15 \\
& * \text { Job Type }+\beta_{16} * \text { Sex }+\beta_{17-20} * \text { Education }+\beta_{21,22} \\
& * \text { ICD diagnosis }+\beta_{23} * \text { Region }+e
\end{aligned}
$$

The variables used in the calculation represent the information available during the application process. Some of the variables, in the propensity score model, were not individually statistically significant but the likelihood ratio test statistic for the model was large $($ Prob $>$ chi $2=0.0000)$.

The program impact was calculated using inverse probability weighted regression adjustment. Average treatment effects were calculated to compare the potential outcomes of all rehabilitants under each treatment scenario. In addition, the average treatment effect on the treated of participation in two-year (one-year) re-training programs was estimated against the alternatives of participation in the shorter (longer) re-training. The applied IPWRA estimator ${ }^{22}$ was composed of a three-step approach to estimating treatment effects:

1) probability weights (propensity scores) were estimated (probit)

2) regression models of the outcome for each treatment level were estimated (OLS); observations were weighted by the inverse probability of being treated

3) potential outcome means were predicted under each treatment scenario; the contrast provided an estimate of the ATE. By restricting the analysis to the sub-samples of treated and non-treated subjects, the ATET were estimated.

Additionally, post-estimation statistics were computed to examine whether the covariates were balanced across treatment groups after re-weighting of data. First, the distribution of propensity scores within the two treatment groups was graphed

\footnotetext{
22 The IPWRA method is explained in more detail in the methods section of the previous chapter and in the appendix to Chapter 3 (sections 3.2.6 and 3.5). Here a probit model is used instead of a multinomial logit model because the treatment variable can only take two values.
} 
in order to assess whether the two distributions have enough overlap. Second, the standardized difference in covariates before and after re-weighting of observations were calculated. Standardized differences refer to the difference in the mean value of a variable between treatment groups, divided by the pooled standard deviation. ${ }^{23}$

\subsection{Results}

\subsubsection{Descriptive characteristics of study participants}

In Table 15, the descriptive statistics of the measured covariates are disaggregated by the type of measure provided. The mean age of full re-training participants was substantially lower than that of partial re-training participants. The proportion of women was greater in two-year re-training programs than in one-year programs. Participants of two-year re-training programs also had higher incomes in the years before program admission and were less often unemployed at the time of application than one-year re-training participants.

Whereas only roughly a quarter of full re-training participants resided in one of the former East Germany states, this figure rose to more than half for those in partial retraining participants. The primary medical problems differed to some extent between the different program participants. Compared to one-year re-training participants, two-year re-training participants more often had musculoskeletal and soft-tissue disorders whereas the share of people with mental and behavioral disorders was slightly higher in the one-year re-training group.

Moreover, prior education attainments can be compared; the education variable, however, contained numerous missing values and can, thus, only to a limited extent be used as a valid predictor for prior educational attainments. Nevertheless, including the given information makes sense since education is thought to be a decisive covariate. Most participants for whom education data was available were not highly educated, whereby more full re-training participants than partial retraining participants had a prior apprenticeship. In addition, the registered job position held two years before admission into rehabilitation was used as a further covariate. Re-training participants, who were employed, mainly carried out qualified manual tasks or were employed in (semi-) professions, followed by simple manual labor and services.

\footnotetext{
${ }^{23}$ The calculation of standardized differences is explained in more detail in the methods section of the previous chapter (Section 3.2.6).
} 
Table 15: Baseline characteristics of one-year and two-year re-training participants (including program dropouts)

\begin{tabular}{lll}
\hline Baseline characteristics & $\begin{array}{l}1 \text {-year re-training } \\
(n=317)\end{array}$ & $\begin{array}{l}\text { 2-year re-training } \\
(n=2,082)\end{array}$ \\
\hline $\begin{array}{l}\text { Age in years } \\
\quad \text { Mean (SD) }\end{array}$ & $41.55(6.89)$ & $37.85(6.76)$ \\
$\begin{array}{l}\text { Gender } \\
\quad \text { Females }\end{array}$ & $33 \%$ & $36 \%$ \\
$\quad \begin{array}{l}\text { Income 2003 } \\
\quad \text { Mean (SD) }\end{array}$ & $€ 9,845(€ 11,692)$ & $€ 12,963(€ 12,393)$ \\
$\quad \begin{array}{l}\text { Income 2004 } \\
\quad \text { Mean (SD) }\end{array}$ & $€ 5,702(€ 9,129)$ & $€ 6,666(€ 9,979)$ \\
$\quad \begin{array}{l}\text { Employment Status } \\
\quad \text { Unemployed }\end{array}$ & $61 \%$ & $45 \%$ \\
$\quad \begin{array}{l}\text { Residential Region } \\
\quad \text { Former East Germany }\end{array}$ & $56 \%$ & $28 \%$ \\
$\quad \begin{array}{l}\text { Medical Diagnosis } \\
\text { ICD 5 (Mental Disorders) }\end{array}$ & $16 \%$ & $14 \%$ \\
$\quad$ ICD 13 (Musculoskeletal Disorders) & $57 \%$ & $64 \%$ \\
\hline
\end{tabular}

Notes: Additional control variables used in the analysis but not reported in this output are indicator variables for the level of education and the last registered occupation type. For both groups of variables, there were many observations with missing data. Most individuals in the sample for whom education data was available have no tertiary education, but many have completed an apprenticeship. The individuals in the sample for whom data on the previous employment type was available, were most frequently formerly employed in qualified manual tasks, (semi-) professions as well as simple manual labor and services.

\subsubsection{Covariate balance}

The average propensity of program participants to have been selected into the oneyear program is summarized in Table 16. Accordingly, the average propensity score of being in the one-year program was (by design) higher for one-year re-training participants than for two-year re-training participants. However, the difference was relatively small, and for the full range between minimum and maximum values, comparable candidates were found in the comparison group. Furthermore, the common support condition was assessed by visualizing the distribution of treatment densities. The overlap between treatment propensities is graphed in Figure 6. For all observations in the re-training program, there were at least some candidates in the other program who have a propensity score in a similar range. After re-weighting the data, the two distributions almost perfectly overlap, indicating good performance of the applied re-weighting estimator. 
Table 16: Overview propensity scores one- and two-year participants

\begin{tabular}{llllll}
\hline Group & Observations & $\begin{array}{l}\text { Mean } \\
\text { P-score }\end{array}$ & $\begin{array}{l}\text { Std. } \\
\text { Dev }\end{array}$ & $\begin{array}{l}\text { Min } \\
\text { P-score }\end{array}$ & $\begin{array}{l}\text { Max } \\
\text { P-score }\end{array}$ \\
\hline 1yr participants & 317 & 0.22 & 0.13 & 0.01 & 0.66 \\
2yr participants & 2082 & 0.12 & 0.09 & 0.00 & 0.67 \\
\hline
\end{tabular}

Notes: Dependent variable $=1$ if rehabilitant was allocated into one-year re-training

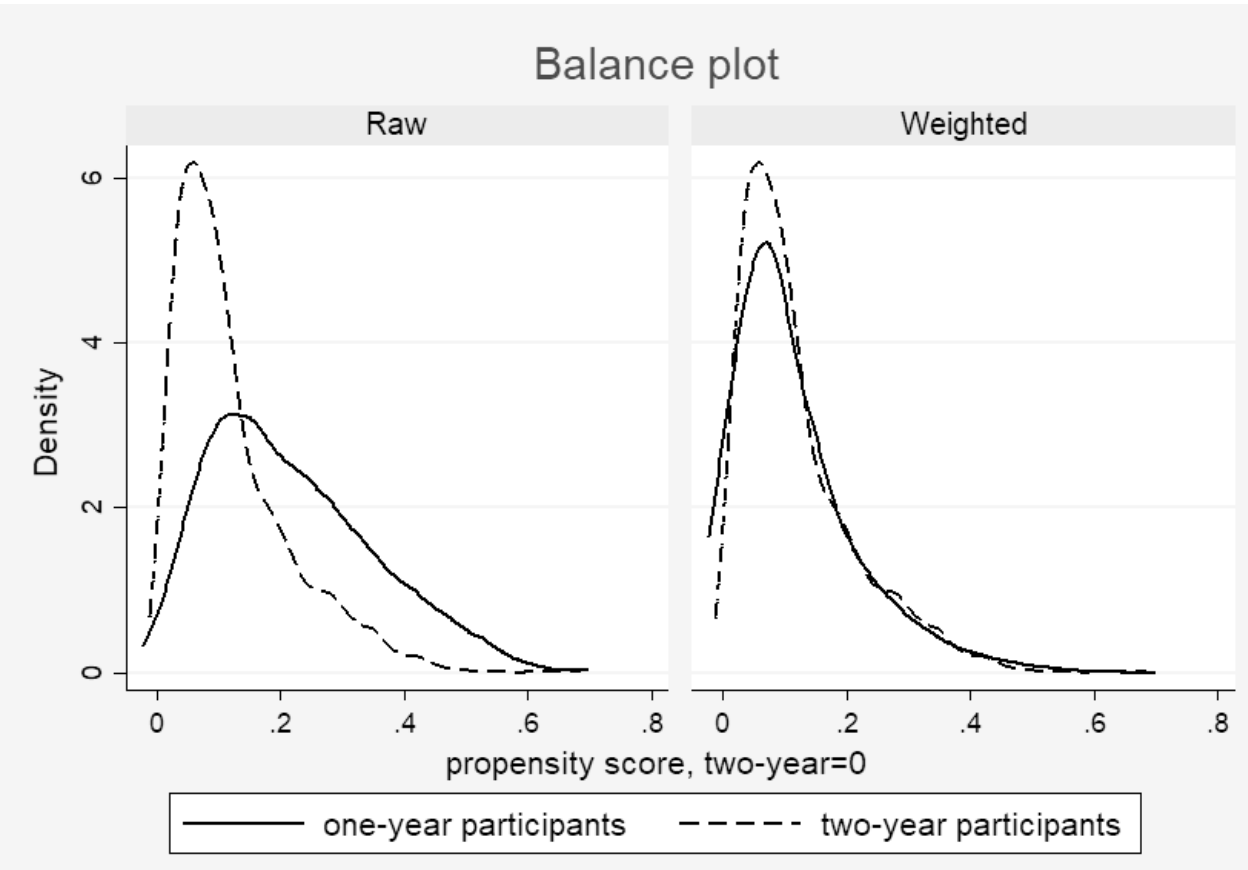

Figure 6 Overlap plot of propensity scores full re-training vs. partial re-training

Table 17 shows that the mean standardized differences in covariates between the participants of one- and two-year re-training can be considered small, after reweighting observations, when compared to a cut-off of 0.10 standard deviations. For example, the standardized difference in age was reduced from 0.54 standard deviations to 0.08 standard deviations, while that of the difference in income earned in 2003 was reduced from 0.26 to 0.04 . The largest improvement in balance was attained for the region variable, where the standardized difference was reduced from 0.58 to 0.03 . Summarizing, the analysis of standardized differences suggests that the distribution of covariates between comparison groups were balanced after observations were re-weighted by the inverse probability of being treated. 
Table 17: Standardized differences in covariates before and after re-weighting of observations, one-year re-training participants vs. two-year re-training participants

\begin{tabular}{lrr}
\hline & Initial sample & Weighted Sample \\
\hline 1 yr & $n=317$ & $n=1,218$ \\
2 yr & $n=2,082$ & $n=1,181$ \\
\hline Baseline characteristics & 1yr vs. $2 \mathrm{yr}$ & 1yr vs. 2yr \\
\hline Age & -0.54 & 0.08 \\
Female & 0.07 & -0.01 \\
Income 2003 & 0.26 & 0.04 \\
Income 2004 & 0.10 & -0.04 \\
Income 2004 * Age & 0.04 & -0.03 \\
Income 2003 * Income 2003 & 0.21 & 0.01 \\
Unemployed & -0.33 & 0.10 \\
Former East Germany & -0.58 & 0.03 \\
ICD 5 (Mental Disorders) & -0.05 & -0.04 \\
ICD 13 (MSD) & 0.14 & 0.06 \\
Neither Abitur nor apprenticeship & -0.03 & 0.03 \\
No Abitur but apprenticeship & 0.16 & -0.10 \\
Abitur & 0.04 & -0.03 \\
University Degree & -0.06 & -0.03 \\
Agriculture & -0.06 & -0.03 \\
Simple Manual Labor & -0.05 & 0.07 \\
Qualified Manual Labor & 0.13 & 0.09 \\
Technician/Engineer & -0.10 & 0.01 \\
Simple Services & -0.04 & 0.04 \\
Qualified Services & 0.10 & 0.07 \\
(Semi-) Professions & 0.26 & -0.03 \\
Simple commercial/administrative & -0.07 \\
Qualified commercial/administrative & 0.02 & 0.00 \\
\hline Nots: & -0.01 & \\
\hline
\end{tabular}

Notes: Initial Sample, standardized differences in covariates between comparison groups as measured in data; Weighted Sample, standardized differences in covariates between comparison groups after re-weighting of observations by the inverse probability of being treated. 


\subsubsection{Treatment effects Average Treatment Effects}

Table 18 presents an overview of the estimated accumulative income and employment effects of two-year compared to one-year programs for the evaluation period 2006-2013 using the IPWRA estimator.

The ATE of two-year re-training in comparison to one-year re-training equaled $€ 10,126$ in nominal euros and $€ 8,792$ in 2005 real euros. The results were a consequence of the higher accumulative days in employment after eight years despite longer program duration and higher dropout rates. In relation to the potential outcome mean with one-year re-training, this change in income equaled an increase of about $10 \%$. The estimated accumulative number of days with employment was 85 days higher with two-year re-training compared to participation in one-year re-training. However, the estimated differences in accumulative income and employment days were not statistically significant at the five percent level.

Additionally, the effects on days with unemployment and days with other social benefits were compared. The duration of transfer payments was characterized by shifts. According to the model estimates, participation in a two-year re-training was associated with 241 more days on social security benefits, 38 more days on shortterm unemployment benefits, but 202 fewer days on long-term unemployment benefits. These effects were significant at the one percent level.

Lastly, the likelihood of receiving an earnings incapacity pension was evaluated. With two-year re-training, the likelihood was 3.2\% lower than for those in a oneyear re-training. However, the measured difference in pension receipt was not statistically significant when compared against the significance threshold of five percent.

Taken together, participation in two-year re-training was associated with more days in employment, higher income, significantly fewer days on long-term unemployment benefits and a reduced risk for an earnings incapacity pension, while participation in one-year re-training was associated with significantly fewer days on social security and short-term unemployment benefits. 
Table 18: Potential outcome means and average treatment effects, participation in two-year vs. one-year re-training

\begin{tabular}{|c|c|c|}
\hline & $\begin{array}{l}\text { Potential-Outcome } \\
\text { Mean (SE.) }\end{array}$ & $\begin{array}{l}\text { Average Treatment Effect } \\
\text { 2yr re-training ( } 95 \% \text { CI.) }\end{array}$ \\
\hline \multicolumn{3}{|c|}{ Income (in nominal €) } \\
\hline 1yr re-training & $€ 101,525(€ 5,710)$ & \\
\hline 2yr re-training & $€ 111,651(€ 1,765)$ & $€ 10,126(€-1,327, € 21,578)$ \\
\hline \multicolumn{3}{|l|}{ Income (in $2005 €$ ) } \\
\hline 1yr re-training & $€ 93,774(€ 5,221)$ & \\
\hline 2yr re-training & $€ 102,565(€ 1,624)$ & $€ 8,792(€-1,696, € 19,280)$ \\
\hline \multicolumn{3}{|c|}{ Employment (in days) } \\
\hline 1yr re-training & $1,495(71)$ & \\
\hline 2yr re-training & 1,579 (19) & $85(-58,228)$ \\
\hline \multicolumn{3}{|c|}{ Social Security Benefits (in days) } \\
\hline 1yr re-training & $367(23)$ & \\
\hline 2yr re-training & $608(7)$ & $241(195,287)^{* * *}$ \\
\hline \multicolumn{3}{|c|}{ Short-Term Unemployment (in days) } \\
\hline 1yr re-training & $129(11)$ & \\
\hline 2yr re-training & $167(4)$ & $38(15,61)^{* * *}$ \\
\hline \multicolumn{3}{|c|}{ Long-Term Unemployment (in days) } \\
\hline 1yr re-training & $454(40)$ & \\
\hline 2yr re-training & $251(10)$ & $-202(-284,-122)^{* * *}$ \\
\hline \multicolumn{3}{|c|}{ Earnings Incapacity Pension (percentage) } \\
\hline 1yr re-training & $8.4(1.6)$ & \\
\hline 2yr re-training & $5.3(0.5)$ & $-3.2(-6.6,0.1)$ \\
\hline
\end{tabular}

Notes: Eight-year accumulative results. Long-term UE only from 2006-2010. Inverse Probability Weighted Regression Adjustment Method was used to estimate the potentialoutcome means. The potential-outcome means refer to the average of the outcomes for a specific level of re-training given that all individuals would have attained this outcome. The average treatment effect measures the difference in these means. Final values were rounded to the nearest whole number (to the nearest tenth for percentage). ${ }^{* * *} \mathrm{P}<0.001{ }^{* *} \mathrm{P}$ $<0.01 * \mathrm{P}<0.05$

\section{Average Treatment Effects on the Treated}

Table 19 shows the employment effects associated with participating in two-year retraining in comparison to the counterfactual scenario of participation in a one-year re-training instead (among those that received a two-year re-training). With a oneyear re-training, two-year program participants would have potentially earned $€ 102,650$ over the eight-year observation period. With a two-year re-training, the participants had, on average, earned $€ 11,270$ more. The corresponding ATET on real incomes for two-year program participants was equal to $€ 9,833$. According to the model estimates, two-year program participants had worked an additional 102 days 
compared to having been allocated a one-year re-training. This increase arose despite potential lock-in effects due to longer program duration. However, neither the effect on income nor the effect on employment were statistically significant at the five percent level. With regards to social-security and welfare benefit receipt, participants of two-year programs had 211 fewer days on long-term unemployment benefits but 41 more days on short-term unemployment benefits and 238 more days on other social security benefits as compared to having been allocated into the shorter re-training programs instead. The differences in the number of days with social security and unemployment benefits were significant at the one percent level. In addition, two-year re-training participants had a 3.4\% reduced risk for a pension due to a reduced earnings capacity. The estimate was, however, not signficant at the five percent level.

Table 19: Potential outcome means with one-year re-training among treated individuals with a two-year re-training and average treatment effects on the treated

\begin{tabular}{|c|c|c|}
\hline & $\begin{array}{l}\text { Potential-outcome } \\
\text { mean with one-year } \\
\text { re-training (SE.) }\end{array}$ & $\begin{array}{l}\text { Average treatment } \\
\text { effect on the treated } \\
(95 \% \text { CI. })\end{array}$ \\
\hline \multicolumn{3}{|l|}{ Income (in nominal €) } \\
\hline $2 y r$ re-training participants & $€ 102,650(€ 6,001)$ & $€ 11,270(€-761, € 23,300)$ \\
\hline \multicolumn{3}{|l|}{ Income (in $2005 €$ ) } \\
\hline 2 yr re-training participants & $€ 94,810(€ 5,559)$ & $€ 9,833(€-1309, € 20974)$ \\
\hline \multicolumn{3}{|l|}{ Employment (in days) } \\
\hline $2 y r$ re-training participants & $1,495(76)$ & $102(-51,254)$ \\
\hline \multicolumn{3}{|l|}{ Social Security Benefits (in days) } \\
\hline $2 y r$ re-training participants & $373(24)$ & $238(190,286)^{* * *}$ \\
\hline \multicolumn{3}{|c|}{ Short-Term Unemployment (in days) } \\
\hline $2 y r$ re-training participants & $127(11)$ & $41(18,65)^{* * *}$ \\
\hline \multicolumn{3}{|c|}{ Long-Term Unemployment (in days) } \\
\hline $2 y r$ re-training participants & $451(43)$ & $-211(-296,-125)^{* * *}$ \\
\hline \multicolumn{3}{|c|}{ Earnings Incapacity Pension (percentage) } \\
\hline 2 yr re-training participants & $8.6(1.7)$ & $-3.4(-6.9,0.0)$ \\
\hline \multicolumn{3}{|c|}{$\begin{array}{l}\text { Notes: Eight-year accumulative results. Long-term UE only from } 2006-2010 \text {. Inverse } \\
\text { probability weighted regression adjustment method was used to estimate potential- } \\
\text { outcome means. The potential-outcome means with one-year re-training illustrate the } \\
\text { average potential outcome that would have occurred among those that participated in two- } \\
\text { year re-training had they participated in one-year re-training measures instead. The } \\
\text { average treatment effect on the treated measures the difference in means between the } \\
\text { actual outcome with two-year re-training and the potential outcome with one-year re- } \\
\text { training. Final values were rounded to the nearest whole number (to the nearest tenth for } \\
\text { percentage). }{ }^{* *} \mathrm{P}<0.001{ }^{* *} \mathrm{P}<0.01{ }^{*} \mathrm{P}<0.05\end{array}$} \\
\hline
\end{tabular}


The graph of yearly income differentials (Figure 7) illustrates the development of the relative earnings difference over time, revealing that, after a lock-in period, the relative income of two-year participants was significantly higher in comparison to having participated in a one-year re-training, instead. In the first two years after program admission the earnings differential was negative, due to longer program participation. In the fourth year after program start, the two-year participants were significantly better off compared to the counterfactual scenario of having been allocated a one-year measure. This positive earnings effect persisted until the last year of the observation period, i.e. the year 2013.

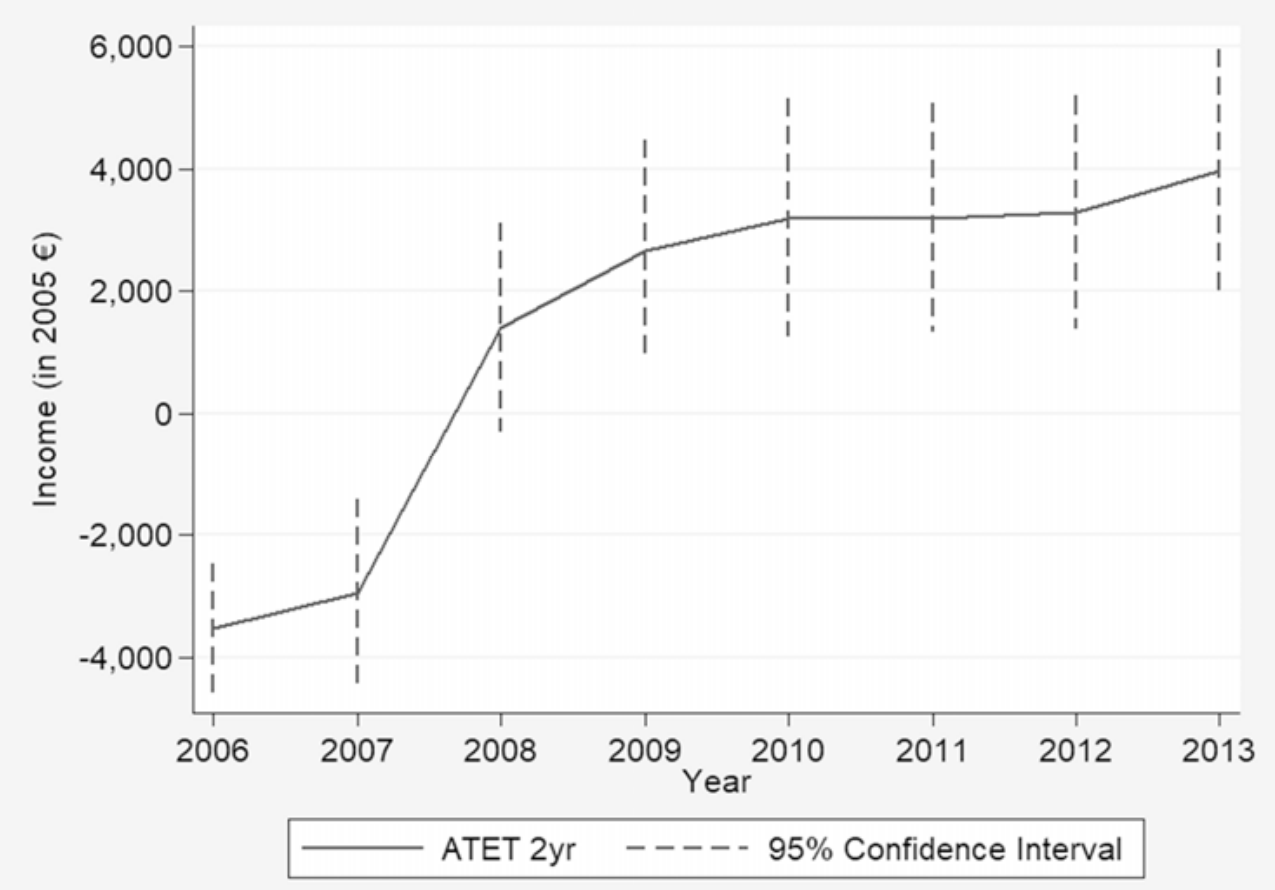

Figure 7 Graphical Analysis of real income development 2006-2013 in comparison to the one-year re-training scenario; average treatment effect on the treated; annual estimates; vertical lines illustrate $95 \%$ confidence interval.

Table 20 shows the employment effects associated with participation in one-year retraining in comparison to the counterfactual scenario of participation in the longer, two-year, re-training (among those that received a one-year re-training). With a twoyear re-training, one-year program participants would have potentially earned $€ 96,406$ over the eight-year observation period. With a one-year re-training, the participants had, on average, earned $€-3,557$ less. The corresponding ATET with regards to inflation-adjusted incomes was equal to $€-2,856$. According to the model 
estimates, one-year program participants had worked an additional 8 days compared to having been allocated a two-year re-training. Neither the effect on income nor the effect on employment were statistically significant at the five percent level. With regards to social-security and welfare benefit receipt, participants of oneyear programs had 164 more days on long-term unemployment benefits but 19 days fewer on short-term unemployment benefits and 264 fewer days on other social security benefits compared to having been allocated into the longer re-training programs instead. The estimated differences in the number of days with social security and long-term unemployment benefits were significant at the one percent level. The effect on days with short-term unemployment benefits was not statistically significant at the five percent level. The small increase in the likelihood to receive an earnings capacity pension, of $0.9 \%$, was also not significant.

Table 20: Potential outcome means with two-year re-training among treated individuals with a one-year re-training and average treatment effects on the treated

\begin{tabular}{ll}
$\begin{array}{l}\text { Potential-outcome } \\
\text { mean with two-year } \\
\text { re-training (SE.) }\end{array}$ & $\begin{array}{l}\text { Average treatment effect } \\
\text { on the treated }(95 \% \mathrm{CI} .)\end{array}$ \\
\hline
\end{tabular}

\begin{tabular}{|c|c|c|}
\hline \multicolumn{3}{|l|}{ Income (in nominal €) } \\
\hline 1yr re-training participants & $€ 96,406(€ 3,025)$ & $€-3,557(€-12,630, € 5,516)$ \\
\hline \multicolumn{3}{|l|}{ Income (in $2005 €$ ) } \\
\hline 1yr re-training participants & $€ 88,601(€ 2,795)$ & $€-2,856(€-11,260, € 5,548)$ \\
\hline \multicolumn{3}{|l|}{ Employment (in days) } \\
\hline 1yr re-training participants & $1,465(33)$ & $8(-111,127)$ \\
\hline \multicolumn{3}{|c|}{ Social Security Benefits (in days) } \\
\hline 1yr re-training participants & $590(11)$ & $-264(-302,-226)^{* * *}$ \\
\hline \multicolumn{3}{|c|}{ Short-Term Unemployment (in days) } \\
\hline 1yr re-training participants & $159(7)$ & $-19(-43,5)$ \\
\hline \multicolumn{3}{|c|}{ Long-Term Unemployment (in days) } \\
\hline 1yr re-training participants & $323(19)$ & $164(93,235)^{* * *}$ \\
\hline \multicolumn{3}{|c|}{ Earnings Incapacity Pension (percentage) } \\
\hline 1yr re-training participants & $6.3(1.0)$ & $0.9(-2.4,4.2)$ \\
\hline \multicolumn{3}{|c|}{$\begin{array}{l}\text { Notes: Eight-year accumulative results. Inverse probability weighted regression } \\
\text { adjustment method was used to estimate potential-outcome means. The potential-outcome } \\
\text { means with two-year re-training illustrate the average potential outcome that would have } \\
\text { occurred among those that participated in one-year re-training had they participated in } \\
\text { two-year re-training measures instead. The average treatment effect on the treated } \\
\text { measures the difference in means between the actual outcome with one-year re-training } \\
\text { and the potential outcome with two-year re-training. Final values were rounded to the } \\
\text { nearest whole number (to the nearest tenth for percentage). }{ }^{* * *} \mathrm{P}<0.001^{* * \mathrm{P}<0.01}{ }^{*} \mathrm{P}<0.05\end{array}$} \\
\hline
\end{tabular}




\subsection{Discussion}

This chapter has provided an econometric evaluation of competing vocational training strategies for people with a disability. The main research goal was to examine the extent to which one- and two-year re-training exhibit similar employment effects. The measured outcomes included changes in earnings, days with employment, days on unemployment and other social-security benefits and the likelihood of receiving a pension due to a reduced earnings capacity.

In the descriptive statistics section, it has been shown that the sampled participants of one-year training measures were older, more often unemployed at program admission and disproportionally more often lived under unfavorable labor market conditions compared to other re-training participants. However, there was also substantial overlap with regards to many of the measured covariates, which was a key prerequisite in order to perform the impact analysis. Inverse probability weighted regression adjustment was used to balance the covariates across comparison groups in order to establish comparability. Subsequently, earnings differentials between participants of full re-training programs and partial re-training programs were estimated.

By prolonging the follow-up in comparison to previous research, the more involved, two-year re-training programs were found to be more effective regarding long-term work participation outcomes as one-year vocational re-training programs. Although two-year re-training measures require an additional year of education, the average treatment effect over the first eight years after program admission was positive; i.e. participation in two-year re-training was associated with more income compared to participation in one-year re-training only, despite an additional year of program duration.

Over the first eight years after program admission, the average treatment effect of two-year re-training compared to one-year re-training, for the whole study population, equaled $€ 10,126,95 \% \mathrm{CI}[€-1,327, € 21,578]$. Furthermore, participation in two-year re-training was associated with more days in employment, fewer days on long-term unemployment benefits and a reduced risk for an earnings incapacity pension. On the other hand, participation in one-year re-training was associated with significantly fewer days on social security benefits and on short-term unemployment benefits.

Since not all individuals can be considered eligible for the longer duration measures, separate analyses of the ATET for the comparison groups were carried out. The subgroup analysis showed, to which extent the two-year participants benefitted from 
actual program participation in the longer type programs and to which extent the one-year group would have benefitted from the longer duration programs.

The first result of the sub-group analysis was, that, over the eight-year observation period, participation in a two-year vocational re-training program was associated with an $€ 11,270$ higher income, 95\% CI [€-761, €23,300], compared to the income achieved after hypothetically having been allocated into a one-year re-training program instead. The increase in income was a consequence of more accumulative days in employment after eight years despite longer program duration and higher dropout rates. The duration of transfer payments, on the other hand, was characterized by shifts, i.e. being allocated a two-year re-training was linked to fewer days with long-term unemployment benefits but more days with short-term unemployment benefits and more days with other social security benefits. Participants of two-year programs had 211 fewer days with long term unemployment benefits, 95\% CI [-296 days, -125 days], but 41 additional days with short term unemployment benefits, 95\% CI [18 days, 65 days], and 238 additional days with other social security benefits, 95\% CI [190 days, 286 days]. Whereas the increase in days with other social security-benefits was mainly due to the longer program duration, the shift in benefit recipience may have been advantageous, as long-term unemployment seems to have been reduced substantially. In addition, two-year re-training participants had a 3.4\% reduced risk for a pension due to a reduced earnings capacity, 95\% CI [-6.9\%, 0.0\%]. The estimate was, however, not signficant at the five percent level

The second result of the sub-group analysis was, that, over the eight-year observation period, participation in a one-year vocational re-training program was associated with a $€-3,557$ lower income compared to the income achieved after hypothetically having been allocated into a two-year re-training program instead, $95 \%$ CI $[€-12,630, € 5,516]$. Neither the effect on income nor the effect on employment days were statistically significant at the five percent level. In fact, the standard error of the estimate of employment days was substantial indicating that the employment effects were very heterogeneous. With regards social-security and welfare benefit receipt, participants of one-year programs had 164 more days on long-term unemployment benefits, 95\% CI [93 days, 235 days], but 19 fewer days on short-term unemployment benefits, 95\% CI [-43 days, 5 days], and 264 fewer days on other social security benefits, 95\% CI [-302 days, -226 days]. This was in comparison to having been allocated into the longer re-training programs instead. The estimated differences in the number of days with social security and long-term unemployment benefits were significant at the one percent level. The effect on days with short-term unemployment benefits was not statistically significant at the five percent level. 
Moreover, the small increase in the likelihood to receive an earnings capacity pension, of $0.9 \%$, was also not significant, $95 \%$ CI [-2.4\%, $4.2 \%]$.

The results indicate, that program allocation was to some extent effective. The ATET of two-year program participants was larger compared than both the ATE and the ATET of one-year program participants (the latter reflects how much lower the income of the one-year participants was compared to having participated in a twoyear program instead). Based on the calculations, the average participant that was allocated into two-year re-training benefited more from the measures, than the average participant that was allocated a one-year re-training would have had. While the retrieved estimates are based on averages, further explorative analysis is needed to examine the conditions under which participants of one-year programs could profit from increased duration of re-training. The response to treatment seems to be heterogeneous and dependent on the individual situation and preferences of the rehabilitants.

Improving the understanding of the conditions under which allocation into a more involved program could lead to better outcomes requires more information on the complex relationship between the applicant and the provider in the application and screening process. There might be more specific reasons, why one-year participants were allocated the reduced-duration re-training, some of which cannot be accounted for in this study. Program participation depends both on rehabilitant eligibility and on the selection by the person in charge or self-selection by potential participants. Thus, it would be relevant to further explore the perspective of the individual before and during the period of rehabilitation as well as the role of the provider in programmatic decision making. 

5. The return on vocational re-training: costeffectiveness from the perspective of social security 


\subsection{Introduction}

Chapter 3 showed that completing a vocational re-training was associated with improvements in income and reductions in the dependency on social security benefits for the participating individuals. Moreover, Chapter 4 showed that, from the individual perspective, the additional year of education, on average, already pays off within the eight-year observation period. In this chapter, the social security perspective is taken to evaluate how long it takes for investments into re-training to break-even and how large the long-term financial returns are.

While legal and social obligations can largely explain expenditures directed at rehabilitation and reintegration, supporting people in the work reintegration process can also be viewed as an economic decision: Assisting workers in the work reintegration process minimizes the negative impact of disabling injuries and diseases on the overall level of production and, consequently, on social security contribution and tax collection. Moreover, reintegrating a worker back into meaningful work prevents the payment of long-term disability pensions and reduces the payment of unemployment and sickness benefits.

Although rehabilitation and reintegration measures are economically important investments, debates about the high costs of such measures often dominate public discussions. A common feature of the costs of work disabilities is that they accrue over a long period of time and among various stakeholders. The difficulties associated with evaluating what the real gains of investing in return-to-work and work reintegration programs lead to individual stakeholders tending to underinvest in workplace reintegration measures, focusing instead on the individual perspective. To assess whether vocational re-training is a worthwhile investment, also from a financial perspective, it is necessary to itemize the services and their resulting costs.

To date, there have been few systematic cost-benefit analyses that evaluate the costeffectiveness of vocational re-training programs from a provider perspective. Such cost-benefit analyses require - in addition to estimates of the impact - information on the specific cost of programs and on the social security system, such as contribution and tax rates or the benefit level of transfer systems.

Not all variables that may be relevant for performance measurement or for calculating an indicator can be collected directly from the routinely generated datasets. Due to the fragmented social security system in Germany with multiple stakeholders, not all data relevant for such a calculation can be accessed through the pension insurance fund research data center. Problems exist, for example, in the 
availability of direct cost data such as the direct program and training costs since the providers of rehabilitation and the implementing organization are not the same.

Some authors of German impact analyses in vocational re-training have tried to perform a cost-benefit analysis or an investigation of the program costs. For instance, in a recent evaluation, Neumann \& Lenske (2010) examined the costs and benefits of vocational rehabilitation for young people with disabilities. The authors conclude that, medium to long term, investment in vocational training for young people with a disability pays off.

In this chapter, the potential economic effects of vocational re-training on public budgets are compared to their costs. The primary objective of this chapter is to investigate the long-term economic impact of vocational re-training on social security providers' balance sheets. While considering consumed resources and economic variables such as inflation, the costs and benefits of one-year and two-year vocational rehabilitation programs to social security are calculated. In addition, the question of one-year or two-year re-training is re-evaluated from the social security perspective

The remainder of this chapter is structured as follows: In the next section, the methods are presented, with a description of the study design, the method of extrapolating benefits and the method of monetarization of indirect benefits. Moreover, a summary of the outcome measures used is given. In the results section, costs and benefits of re-training are evaluated separately before the costeffectiveness for social security systems is assessed. The evaluation includes a sensitivity analysis that also introduces a social discount rate and evaluates the effect of using alternative forecasting models. The results section is concluded by an analysis of the incremental effects of the two-year re-training. In the final section of this chapter, the implications and shortcomings of the findings are discussed before conclusions are drawn.

\subsection{Methods}

\subsubsection{Study design and population}

To investigate whether vocational re-training was cost-effective from the social security perspective, an analysis of the secondary effects of the measures was conducted. This analysis included comparing the financial returns to the social security system with the program costs. The chosen timeframe for the analysis of program returns to social security reflects the average number of years that the average rehabilitant was away from retirement ( 25 years). The benefits of retraining to the social security system were examined based on the results of the 
quasi-experiments in chapters 3 and 4 . Costs were evaluated based on a combination of information retrieved from the primary data used throughout this thesis and information from secondary literature.

\subsubsection{Calculation of benefits to social security Extrapolation of direct benefits}

In this section, predictions about the development of income and social security benefit receipt in the years after the end of the observation period are presented in order to assess the long-term financial returns to the programs. Predicting population outcomes for groups of the same family is less of a challenge than it is for an isolated case. This difference arises because the behavior of the aggregate data by pooling the variances of the population-related factors (which are generally common and well-known) can have stable properties, even if the individuals inside have a high degree of randomness. It is, therefore, easier to achieve a higher degree of accuracy in predicting certain employment outcomes when using pooled population data compared to using data for specific individuals.

In this study, a four-year weighted moving average was employed to model the long-term developments of earnings and days with unemployment benefits. The dynamic four-year weighted moving average took the following general form:

$\mathrm{y}_{\mathrm{t}}=0.1{ }^{*} \mathrm{y}_{\mathrm{t}-4}+0.2{ }^{\mathrm{y}-3}+0.3 * \mathrm{y}_{\mathrm{t}-2}+0.4^{*} \mathrm{y}_{\mathrm{t}-1}$

First, annual treatment effects were estimated for all outcomes. In a next step, using the dynamic specification above, the average treatment effects were extrapolated until the year $2030^{24}$ to provide an estimate of the employment effects of vocational re-training measures over the entire working life. Consequently, accumulative effects for the average program graduate, or participant, were calculated. Table 21 shows the real income differences used in the extrapolation process. Since only the last four years observed were used to predict the future development, the extrapolation results were independent of the initial lock-in and transitional period.

Table 21: Annual income difference 2006-2013

\begin{tabular}{lllllllll}
\hline & 2006 & 2007 & 2008 & 2009 & 2010 & 2011 & 2012 & 2013 \\
\hline 1vs0 & $€ 3,275$ & $€ 4,347$ & $€ 3,508$ & $€ 2,756$ & $€ 2,435$ & $€ 1,677$ & $€ 2,083$ & $€ 2,661$ \\
2vs0 & $€-271$ & $€ 2,263$ & $€ 5,841$ & $€ 5,494$ & $€ 5, \mathbf{1 1 4}$ & $€ 4,528$ & $€ 4,759$ & $€ 5,369$ \\
\hline
\end{tabular}

Notes: Average treatment effect on the treated on annual basis; cf. figure 4 in chapter three.

24 The likelihood of receiving a pension due to a reduced earnings capacity was not extrapolated; instead it was assumed that, once awarded, the recipient stays on benefits until retirement. 
Figure 8 shows the effect of extrapolation by a dynamic four-year weighted moving average on long-term income. The series became stationary after a few years; the development of earnings entered a relatively constant trend, which was then further extrapolated into the future (excluding the initial phase of volatility).

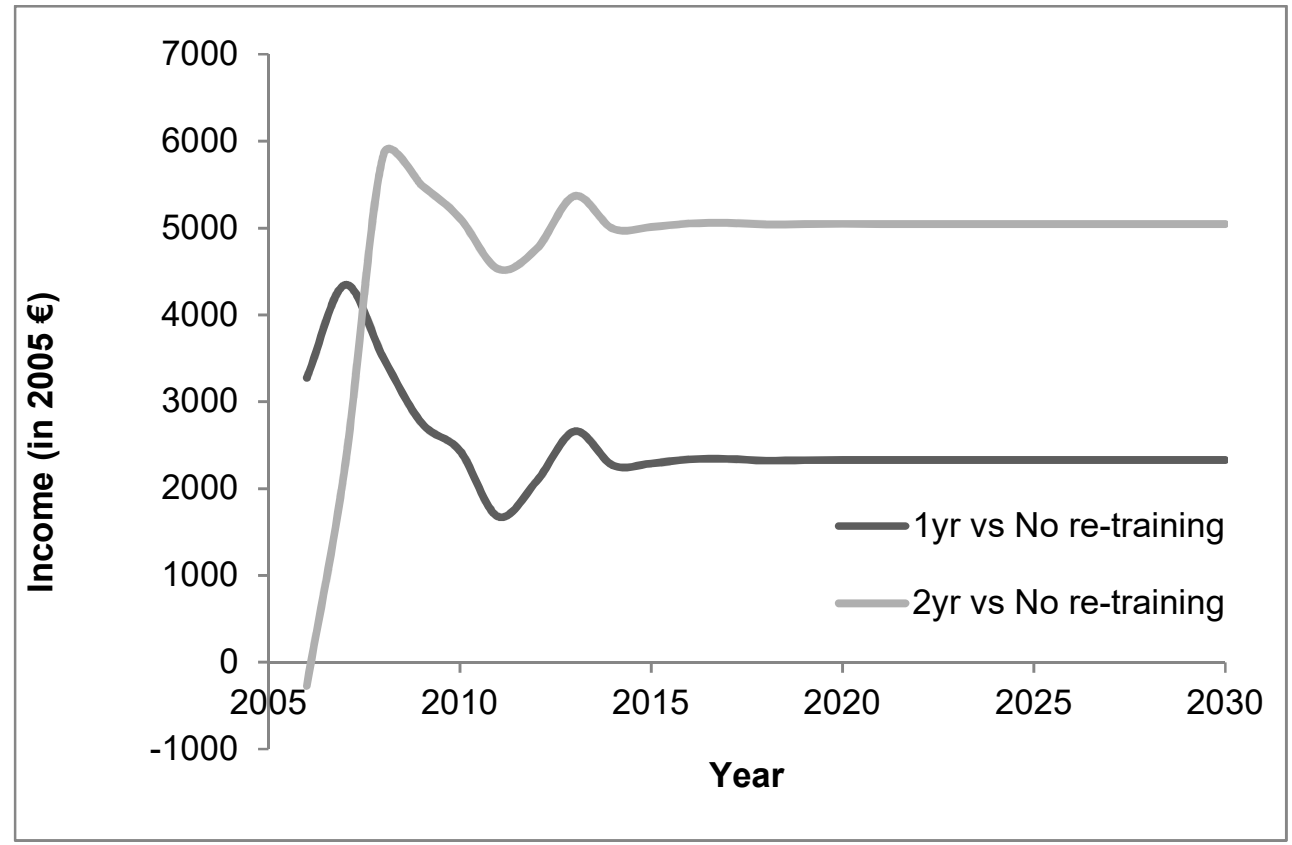

Figure 8 Extrapolation of real wage gains 2013-2030, method of extrapolation: four-year weighted moving average

\section{Monetarization of secondary effects for social security}

Economic benefits can be measured and assessed in different ways. For example, in a cost-effectiveness analysis, consequences are valued in natural units such as prevented employment losses, or the number of sick days saved. In contrast, in a cost-benefit analysis, the concept of value is broadened in order to express the benefits also in monetary terms. Cost-Benefit analysis requires translating the natural units into a monetary value that can be compared with the costs (Drummond, Sculpher, Claxton, Stoddart, \& Torrance, 2015).

The previous section showed the direct effects that can be anticipated from successful re-training and how the effects were extrapolated out of a sample, based on estimated differences. To assess the cost-effectiveness of the measures, the direct benefits, such as the prevented number of days with unemployment, still need to be monetarized so that the benefits can be directly compared to the costs of providing the program. 
Figure 9 illustrates the different direct and indirect outcomes considered. By preventing permanent employment loss ${ }^{25}$ and temporary unemployment, social security systems benefitted from reduced compensation expenditure. Additionally, increased wages mean that taxes and welfare contributions rose. Here, all changes were evaluated in 2005 real Euros (inflation-adjusted).

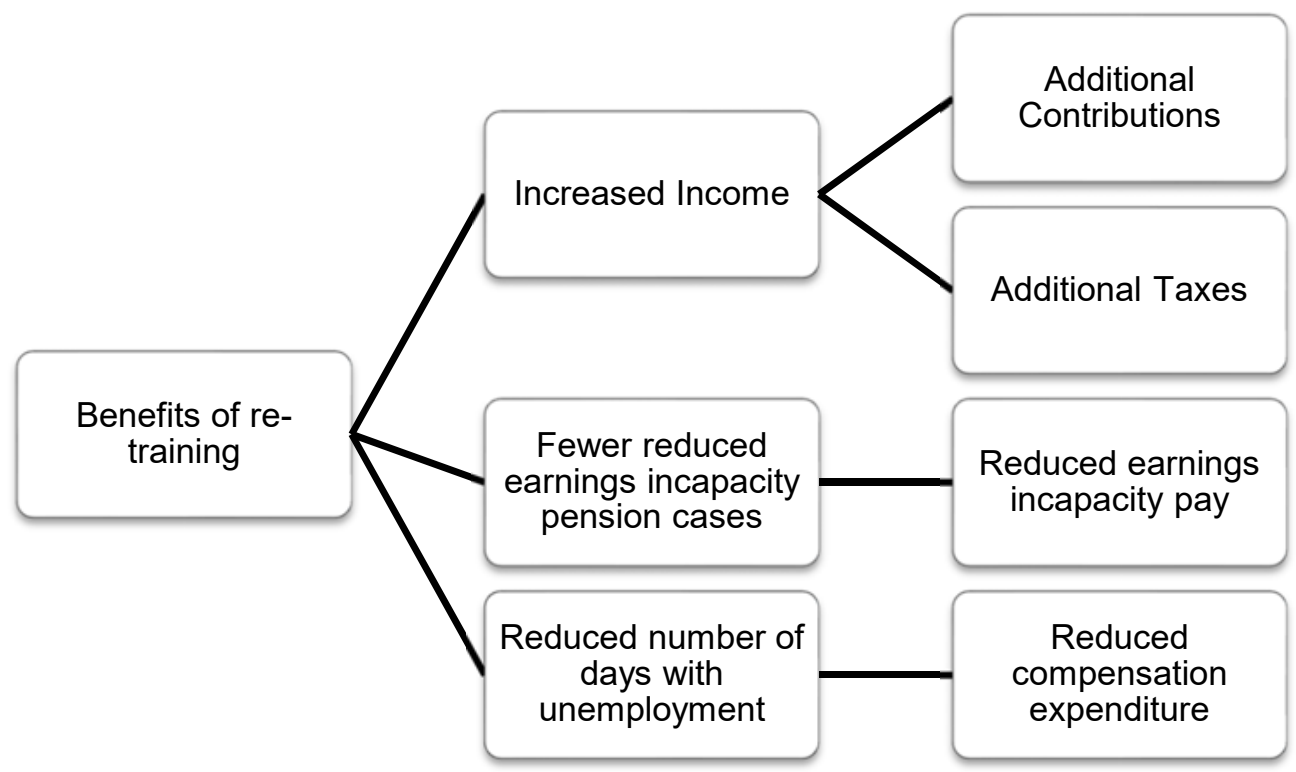

Figure 9 Benefits of re-training to the social security system

Table 22 lists the factors used in calculating the indirect benefits effects resulting from increased income and a reduced number of days on social security benefits. Additional contributions were calculated by multiplying the contribution rate for Germany, which, taken together, equaled $40.75 \%$ with the accumulative gain in real wages.

Additional tax revenue was calculated by factoring in a tax factor, which by assumption equaled $5 \%$ in the baseline scenario. The size of the assumed tax rate was based on a careful evaluation of the tax schedule in Germany. The income tax

\footnotetext{
${ }^{25}$ In the estimation, the reduced risk of receiving a disability pension was not considered to come into effect immediately. Instead annual estimates were retrieved and used in the calculation of the reduced earnings incapacity pay .
} 
in Germany is progressive: first, income tax rates start at $14 \%$, then they rise incrementally to $42 \%$. However, the first $€ 9,168$ (or $€ 18,336$ for married couples submitting a combined return) earned each year are tax free and a considerable number of taxpayer's costs can be deducted from income when computing taxable income (§32a $\mathrm{EStG}^{26}$ ). Given the rather low average income of the rehabilitants (cf. table $12 \& 13)$, a conservative income tax rate was chosen in order to not overestimate the effect on income taxes.

Moreover, unemployment (UE) benefits reduction was estimated by multiplying the respective reduction in days on unemployment and welfare benefits with the respective benefit levels. According to the Statistical Office of the Federal Employment Agency, the level of short-term UE benefits paid in 2005 was, on average, $€ 770$ while the level of long-term UE benefits was $€ 621$. The level of other welfare benefits for that year cannot accurately be measured since it combines various welfare payments such as workers' compensation, sick pay, transitional payments, etc. To, nevertheless, be able to perform the calculation, the level of the other social security benefits was set equal to that of short-term UE benefits, $€ 770$. Taken together, these estimates provided the basis for calculating the benefits for social security providers.

Table 22: Factors used to monetarize the benefits of vocational re-training

\begin{tabular}{|c|c|c|}
\hline Factor & Value & Source \\
\hline \multicolumn{3}{|l|}{ Benefits } \\
\hline $\begin{array}{l}\text { Social security } \\
\text { contribution rate }\end{array}$ & $40.75 \%$ & $\begin{array}{l}\text { International Social Security } \\
\text { Association (2014) }\end{array}$ \\
\hline $\begin{array}{l}\text { Effective income tax } \\
\text { rate }\end{array}$ & $5.00 \%$ & Assumed, $0 \% / 10 \%$ sensitivity \\
\hline Average Pension size & $€ 606$ per month & Primary data (was in dataset) \\
\hline Short-term UE & $€ 770$ per month & $\begin{array}{l}\text { Bundesagentur für Arbeit } \\
(2006, \text { p. 76) }\end{array}$ \\
\hline Long-term UE & $€ 621$ per month & $\begin{array}{l}\text { Bundesagentur für Arbeit } \\
(2006, \text { p. } 78)\end{array}$ \\
\hline Other welfare benefits & $€ 770$ per month & $\begin{array}{l}\text { Assumed, combines various } \\
\text { payments }\end{array}$ \\
\hline
\end{tabular}

\subsubsection{Cost of providing rehabilitation for social security}

Figure 10 illustrates the different costs of re-training considered in this analysis. Three main cost drivers were considered in this analysis that determine the size of

\footnotetext{
${ }^{26}$ German Income Tax Act
} 
the investment made by the social security system. These were the training costs, income replacement benefits provided and, in some circumstances, housing benefits for residence in a boarding school.

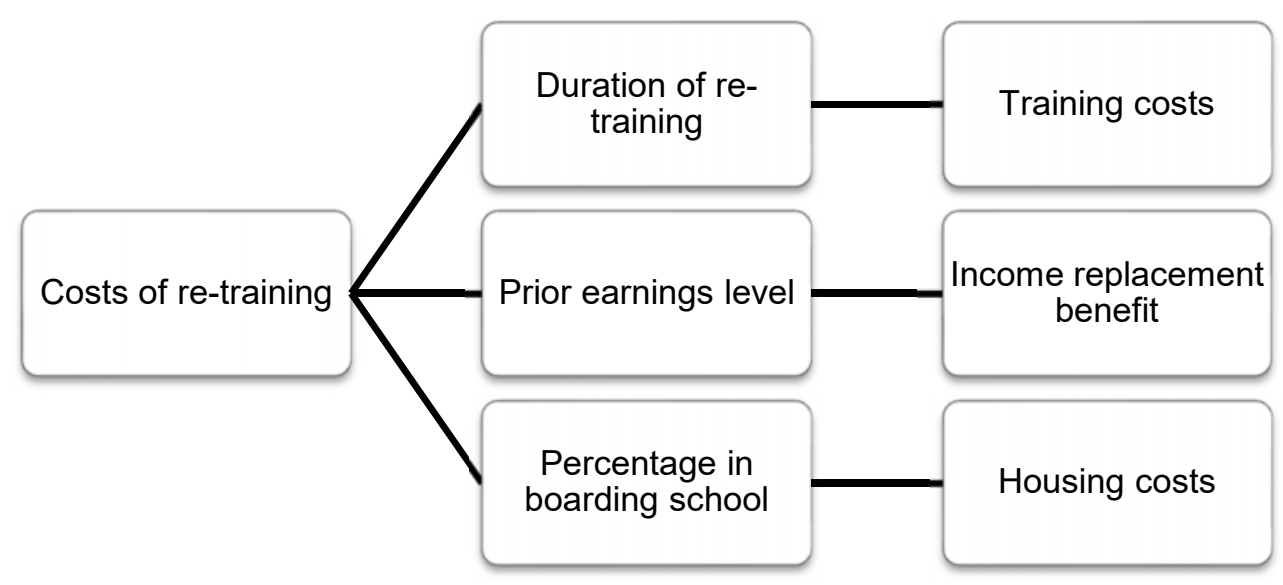

Figure 10 Costs of re-training to the social security system

Table 23 lists the factors used in calculating the costs of re-training. According to information from the German Statutory Pension Insurance Fund, direct costs of retraining are equal to about $€ 1,500$ per month. If a residential placement is required, additional expenses of $€ 500$ per month apply (Kardorff \& Ohlbrecht, 2013). Additionally, to cover the basic costs of living, an income replacement benefit (subsistence allowance) is paid to the rehabilitants. These values were recorded in the dataset at hand. The average income replacement benefit paid during the period of rehabilitation was equal to $€ 8,933$ for one-year program graduates and $€ 22,192$ for two-year program graduates.

Table 23: Factors used to monetarize the costs of vocational re-training

\begin{tabular}{lll}
\hline Factor & Value & Source \\
\hline Costs & & \\
Training Costs & $€ 1,500$ per month & Kardorff \& Ohlbrecht (2013) \\
Housing Costs & $€ 500$ per month & Kardorff \& Ohlbrecht (2013) \\
Income Replacement & 1yr grad: $€ 8,933$ & Primary data (in dataset) \\
& 2yr grad: $€ 22,192$ & Primary data (in dataset)
\end{tabular}




\subsubsection{Cost-effectiveness outcome metrics}

The following section summarizes the outcomes used to evaluate the costeffectiveness of the re-training programs.

\section{Break-even analysis}

The breakeven point (in years) is the point where the financial benefits and costs of re-training are the same. After the breakeven point is exceeded, the social security system incurs a profit; i.e. the net present value (PV) becomes positive. Since the measure is independent of the extrapolation period chosen, it is the primary outcome metric in this analysis.

Breakeven point: $P V$ Benefits $=$ PV Costs

Net present Value (NPV)

The net present value (NPV) is used to compare the cash flows occurring at different times resulting from re-training. The present value of cash flow depends on the time interval up to the cash flow and on the chosen discount rate. The present value, thus, considers the time value of the money. Since, in the baseline scenario, inflation is already accounted for by using real values only, the net present value equals the accumulative sum of the estimated outcomes for the years 2006-2030. In the sensitivity analysis, the effect of introducing a social discount rate is analyzed. With discounting, the net present value is calculated as follows:

$N P V: \sum_{t=1}^{T} \frac{\text { Benefits }_{t}}{(1+i)^{t}}-$ Initial Investment

\section{Return On Investment (ROI)}

The return on investment (ROI) is the ratio between the net profits and the costs that result from the investment into the re-training program. A high ROI, i.e. above 1, means that the investment has a positive overall financial impact. As a measure of performance, the ROI is used to assess the efficiency of an investment or to compare the efficiency of several different investments.

ROI $=\frac{\text { PV Benefits }}{\text { PV Costs }}$

Internal Rate of Return (IRR)

The IRR is the discount rate that sets the net present value of all cash flows from a given project equal to zero. In this study, since all costs and benefits are already measured in real terms, the factor can be interpreted as the rate at which future benefits would have to been discounted, above current interest rates, for the programs to no longer be cost-effective; i.e. the net present value becomes negative. 


\subsection{Results}

In this section, the effects on taxes, contributions and compensation payments are compared to the cost of the interventions. Before the final outcomes are presented, the different costs and benefits of the re-training programs, as calculated, are examined individually.

\subsubsection{Benefits of one-and two-year re-training for social security}

Table 24 shows the results of the extrapolation process. These results can be considered the predicted overall direct effect of the programs over the rehabilitants' working lives. According to the model estimation, accumulative wage effects over the entire period (including the extrapolation/forecast period) were equal to $€ 62,245$ and $€ 118,826$, for one- and two-year programs, respectively.

With regards to benefit receipt, one-year program graduates were predicted to have, 141 fewer days on short-term UE benefits, 90 fewer days on long-term UE benefits and 539 fewer days on other social security benefits. Two-year program graduates were predicted to have 105 fewer days on short-term UE benefits, 710 fewer days on long-term UE benefits and 378 fewer days on other social security benefits.

While income and days with unemployment and other social security benefits were forecasted according to the four-year weighted moving average model, the effect on the earnings incapacity pension uptake was held fixed after the eight-year observation period, i.e. in the model, a constant effect corresponding to the recipient level in the last observed year was assumed.

Table 24: Measured and extrapolated effects resulting from program completion (method of extrapolation: last four-years weighted moving average)

\begin{tabular}{lcccc}
\hline & \multicolumn{2}{c}{$2006-2013$} & \multicolumn{2}{c}{$2006-2030$ (with forecast) } \\
\hline Variable & $1 \mathrm{yr}$ & $2 \mathrm{yr}$ & $1 \mathrm{yr}$ & $2 \mathrm{yr}$ \\
\hline Additional wages & $€ 22,742$ & $€ 33,097$ & $€ 62,245$ & $€ 118,826$ \\
Reduction short-term UE & -26 days & -24 days & -141 days & -105 days \\
Reduction long-term UE & +6 days & -225 days & -90 days & -710 days \\
Reduction social security & -316 days & -150 days & -539 days & -378 days \\
Earnings incapacity & $-3.1 \%$ & $-6.0 \%$ & $-3.1 \%$ & $-6.0 \%$ \\
\hline
\end{tabular}

Notes: Long-term UE measurement only from 2006-2010, '11-13 was extrapolated. For the two-year intervention group, the 2006 Social Security estimate was disregarded to prevent double-counting of the income replacement benefit provided (income replacement benefit was part of the direct costs as measured). The effect on earnings incapacity was held fixed. 
Table 25 provides an overview of the financial benefits to social security. These comprise additional social security contributions and taxes collected, prevented pay of (short- and long-term) unemployment and other welfare benefits, as well as prevented disability pension payments. The benefits were estimated by comparing them to the counterfactual scenario of non-completion of the measures. This study takes a holistic approach without segregation into the different social security branches: pension, health, unemployment and care insurance.

According to our estimates, the largest benefits to social security were additional contributions collected, which were the result of the program graduates' increased incomes. For one-year re-training graduates, long-term contribution collection rose by $€ 25,365$ per individual that successfully completed a program; for two-year vocational re-training by $€ 48,422$. Income taxes to the federal government rose by $€ 3,112$ and $€ 5,941$, respectively. Additionally, by preventing UE, expenditure from compensation payments was additionally prevented. Short-term UE savings were equal to $€ 3,574$ (one-year) and $€ 2,661$ (two-year). The long-term UE benefit savings were $€ 1,844$ (one-year) and $€ 14,488$ (two-year). Savings due to fewer days on social security benefits totaled $€ 13,648$ for one-year programs and $€ 9,566$ for two-year programs, on average. Additionally, due to fewer cases of earnings incapacity pension in comparison to the controls, the average value paid out was reduced by $€ 4,587$ and $€ 8,882$, for one-year and two-year programs, respectively. These numbers reflect the average gain linked to successful rehabilitation per individual. The value of a single case of prevented disability pension was, of course, much higher.

The overall costs to social-security, thus, accumulated to $€ 52,129$ for one-year retraining and $€ 89,959$ for two-year re-training.

Table 25: Benefits to social security for average program completer (2006-2030)

\begin{tabular}{lll} 
Benefits to Social Security & 1yr re-training & 2yr re-training \\
\hline Additional contributions & $€ 25,365$ & $€ 48,422$ \\
Additional taxes & $€ 3,112$ & $€ 5,941$ \\
Short-term UE & $€ 3,574$ & $€ 2,661$ \\
Long-term UE & $€ 1,844$ & $€ 14,488$ \\
Social security benefits & $€ 13,648$ & $€ 9,566$ \\
Reduced earnings incapacity pension & $€ 4,587$ & $€ 8,882$ \\
\hline Overall & $€ 52, \mathbf{1 2 9}$ & $€ 89,959$
\end{tabular}

Notes: Ctr. $=40,75 \%$, tax $=5 \%$, daily short-term UE benefit $=€ 25.32$ ( $€ 770$ per month), daily long-term UE benefit $=€ 20.42(€ 621$ per month), daily social security benefit $=€ 25.32(€ 770$ per month), yearly pension $=€ 7,724$ (€606 per month) 


\subsubsection{Costs of one-and two-year re-training for social security}

To determine whether the benefits from rehabilitation were cost-effective, information on expenditure is needed to put the costs and benefits into perspective. There are three main cost drivers that determine the size of the investment made by the social security system: training costs, income replacement benefits and, in some circumstances, housing benefits for residence in a boarding school.

Since these costs directly depend on program duration, the average program duration among the two treatment groups was evaluated. Table 26 shows that partial re-training, on average, took 9.72 months while full re-training was usually completed after 21.19 months. The average length of training was retrieved from the dataset at hand. Without a direct measurement, the size of the treatment costs was based on information from the pension insurance published in secondary literature and linked with the actual measurement on the program duration.

Table 26: Program duration of one-year and two-year re-training

\begin{tabular}{lll}
\hline & 1yr re-training & 2yr re-training \\
\hline Duration (in months) & 9.72 & 21.19
\end{tabular}

Since not all rehabilitants require housing, actual usage statistics were retrieved from the dataset used throughout this thesis. Table 27 presents the distribution of housing provisions among treatment groups. Among one-year re-training graduates, $9.35 \%$ were provided with housing in a boarding school during program participation while, among two-year re-training graduates, $25.60 \%$ were provided with a permanent placement in a boarding school.

Table 27: Type of housing provided during rehabilitation

\begin{tabular}{llll}
\hline Housing provision & 1yr re-training & 2yr re-training & Total \\
\hline Boarding School & $26(9.35 \%)$ & $449(25.60 \%)$ & $475(23.38 \%)$ \\
None & $252(90.65 \%)$ & $1,305(74.40 \%)$ & $1,557(76.62 \%)$ \\
\hline Total & $278(100 \%)$ & $1,754(100 \%)$ & $2,032(100 \%)$
\end{tabular}

Table 28 provides a summary of the three costs linked to the provision of vocational re-training accruing to the social security provider. For the average duration of 9.72 and 21.19 months, treatment costs (1,500€ per month) of $€ 14,583$ and $€ 31,791$ applied, respectively. To cover the basic costs of living, an income replacement benefit (subsistence allowance) is usually paid to the rehabilitants. On average, the income replacement benefit paid equaled, $€ 8,933$ for a one-year re-training and $€ 22,192$ for a two-year re-training. Additionally, the average costs of boarding per rehabilitant ( $€ 500$ per month) accumulated to $€ 4,861$ and $€ 10,597$, respectively. However, the housing costs in fact only applied to $9.35 \%$ of all one-year graduates and $25.60 \%$ of 
two-year graduates, in the sample that was evaluated. Accordingly, housing costs per average rehabilitant were included in the final estimate. They equaled $€ 454$ and $€ 2,713$, for one- and two-year programs, respectively.

The overall costs of providing vocational re-training, with a proportionate share of people receiving housing benefits, thus, equaled $€ 23,971$ for one-year of training, and $€ 56,695$ for two-year programs.

Table 28: Average costs to social security for re-training measure provided, per program completer

\begin{tabular}{lll} 
& 1yr re-training & 2yr re-training \\
\hline Treatment costs $(€ 1,500$ per month) & $€ 14,583$ & $€ 31,791$ \\
Income replacement benefit & $€ 8,933$ & $€ 22,192$ \\
(Housing, per case) & $€ 4,861$ & $€ 10,597$ \\
(Housing, per average rehabilitant) & $€ 454$ & $€ 2,713$ \\
\hline Total without housing & $€ 23,516$ & $€ 53,983$ \\
Total with housing & $€ 28,394$ & $€ 64,580$ \\
Total, per average rehabilitant & $€ 23,971$ & $€ 56,695$
\end{tabular}

\subsubsection{Cost-effectiveness of one-year and two-year re-training for social security}

After all benefit types have been monetarized, the outcomes were compared with the initial investment. First, the results without (additional) social discounting are presented as they represent the financial returns to the investment made in the year 2005 (the results were adjusted for inflation).

Table 29 shows that, from the social security perspective, the re-training programs generally pay off. However, the benefits outweighed the costs only after a substantial payback period. On average, investments into one-year re-training measures broke even after 11 years, while investments into two-year measures broke even after 17 years. The calculated social discount rate that set the present value of the benefits equal to the costs equaled $8.16 \%$ for one-year re-training programs and $3.68 \%$ for the two-year re-training programs.

The total net present value after 25 years was equal to $€ 28,159$ for one-year retraining, whereas for two-year re-training, the returns were $€ 33,264$ larger in comparison with program costs.

The corresponding ROI ratios (benefits over costs) equaled 2.17 and 1.59, for oneyear and two-year re-training, respectively. This means that, for every euro invested into one-year re-training, the public receives an average return of $€ 2.17$ in the form 
of additional taxes and contribution generated and compensation pay prevented, in the long run. For every euro spent on two-year re-training, the public receives an average return of $€ 1.59$.

Table 29: Overview of cost-benefits for the average program completer 2006-2030

\begin{tabular}{lll} 
& 1yr re-training & 2yr re-training \\
\hline PV Benefits Program Completer & $€ 52,129$ & $€ 89,959$ \\
PV Costs Program Completer & $€ 23,971$ & $€ 56,695$ \\
\hline NPV Program Completer & $€ 28,159$ & $€ 33,264$ \\
ROI & 2.17 & 1.59 \\
Internal Rate of Return & $8.16 \%$ & $3.68 \%$ \\
Break-Even Point & 2016 & 2022 \\
& $(11$ years $)$ & $(17$ years $)$ \\
\hline
\end{tabular}

Notes: The general approach taken in this analysis of the ROI was that costs-benefit relationships were considered per successful rehabilitation case. Thereby, it was assumed that, in the counterfactual scenario of non-graduation, no intervention or income replacement costs were paid. This assumption provides the basis for constructing costbenefit relationships for the average successful rehabilitation case in comparison to the alternative scenario of no treatment.

\section{Sensitivity analysis}

In this section, the effect of changed input factors on the estimated cost-benefit relationships is tested. This sensitivity analysis includes introducing a social discount rate to evaluate the effects on social security considering intertemporal time preferences, changing the forecasting model to naïve and autoregressive integrated moving average (ARIMA) and altering the assumed income tax rate due to it being the only assumed (freely chosen) variable in the calculation. Alternations in the cost of the social discount rate, the effective income tax rate and the forecasting model used were evaluated with regards to the final cost-effectiveness metrics, Break Even, NPV, ROI, and IRR.

\section{The effect of social discounting}

The different benefits to social security, despite already being formulated in real terms, can be compared in terms of the net value to society using a social discount factor. Following the advice of Drummond et al. (2015) interest rates of $2 \%$ and $5 \%$ were chosen to facilitate comparison with other studies. In Table 30, the effect of a social discount rate on the accumulative wage effect over the 25-year period is shown. With a two percent social discount factor, the income gain of one-year program completers was reduced from $€ 62.245$ to $€ 49.354$. With five percent social discounting, the wage gain was further reduced to $€ 36.515$. For two-year program completers, a two percent social discount factor reduced the accumulative wage 
effect from $€ 118,826$ to $€ 91,371$. With five percent discounting, the wage gain of twoyear completers was further reduced to $€ 64,228$.

Table 30: Effect of social discounting on accumulative income forecast 2006-2030

\begin{tabular}{llll}
\hline & $\begin{array}{l}\text { No social } \\
\text { discounting }\end{array}$ & $\mathrm{r}=1.02$ & $\mathrm{r}=1.05$ \\
\hline 1yr real income gain & $€ 62.245$ & $€ 49.354$ & $€ 36.515$ \\
2yr real income gain & $€ 118,826$ & $€ 91,371$ & $€ 64,228$
\end{tabular}

Table 31 shows the results of social discounting on final outcomes. As with wages, the attained final effects in compensation payments and disability payments have also been discounted using the specified social discount rate.

Compared to the zero social discount rate scenario (intertemporal indifference), with two percent discounting, the payback period of one-year programs was increased by one additional year while the payback period of two-year programs was increased by three years. The net present value and ROI ratios, assessed over the 25year period, were somewhat smaller than the baseline scenario. However, the programs were still cost-effective: with a two percent social discount factor. The net present value of one-year programs, over the 25-year period, decreased from $€ 28,159$ to $€ 17,558$ while the ROI was reduced from 2.17 to 1.73 . The corresponding net present value of the two-year programs was reduced from $€ 33,264$ to $€ 12,669$ while the ROI was reduced from 1.59 to 1.22 .

With an assumed social discount rate of five percent, the payback period of one-year re-training programs increased by five additional years while the two-year programs were estimated to not be cost-effective within the specified period of analysis. The net present value of one-year programs over the 25-year period, with a five percent social discount rate, was equal to only $€ 6,991$ while the ROI was reduced further to 1.29. The corresponding net present value of two-year programs was equal to $€-7,689$ while the ROI was reduced to 0.86 .

Table 31: Sensitivity analysis: Social discount factor

\begin{tabular}{lcccccc}
\hline $\begin{array}{l}\text { Social Discount } \\
\text { Factor }\end{array}$ & \multicolumn{3}{c}{1.00 (baseline) } & \multicolumn{2}{c}{1.02} & \multicolumn{2}{c}{1.05} \\
\hline Group & $1 \mathrm{yr}$ & $2 \mathrm{yr}$ & $1 \mathrm{yr}$ & $2 \mathrm{yr}$ & $1 \mathrm{yr}$ & $2 \mathrm{yr}$ \\
\hline Break-even & 2016 & 2022 & 2017 & 2025 & 2021 & $>2030$ \\
NPV & $€ 28,159$ & $€ 33,264$ & $€ 17,558$ & $€ 12,669$ & $€ 6,991$ & $€-7,689$ \\
ROI & 2.17 & 1.59 & 1.73 & 1.22 & 1.29 & 0,86 \\
IRR & $8.16 \%$ & $3.68 \%$ & - & - & - & -
\end{tabular}




\section{The effect of different forecasting models}

In this section, the extrapolation was re-estimated using two different forecasting models. First, the results attained under a four-year moving average were compared to naïve estimates. In a naïve estimate, the last recorded value, i.e. the outcome from 2013 was held constant over the entire extrapolation period. Second, a first-order autoregressive time series model was used to extrapolate outcomes with a more dynamic process.

Table 32 shows that the four-year weighted moving average is the most conservative forecasting model of the three alternatives tested. Under a naïve estimate, the breakeven amount of years was reduced by one year for each program. The net present value of one-year programs increased from $€ 28,159$ to $€ 35,113$, while the ROI jumped from 2.17 to 2.46 . The estimated IRR increased from $8.16 \%$ to $9.26 \%$.

As a result of a naïve estimate, the net present value of two-year programs increased from $€ 33,264$ to $€ 38,505$ while the ROI increased from 1.59 to 1.68 . The corresponding IRR of the 25 -year period increased from $3.68 \%$ to $4.12 \%$.

With a first-order autoregressive extrapolation process, the number of years needed for the one- and two-year programs to break even was reduced to 10 years and 16 years, respectively. The net present value of one-year programs increased from $€ 28,159$ to $€ 36,599$ while the ROI increased from 2.17 to 2.53 . The estimated IRR increased from $8.16 \%$ to $9.46 \%$.

The net present value of two-year programs increased from $€ 33,264$ to $€ 41,650$ while the ROI increased from 1.59 to 1.73 . The corresponding IRR of the 25-year period increased from $3.68 \%$ to $4.36 \%$.

Table 32: Sensitivity analysis: Forecasting model

\begin{tabular}{lcccccc}
\hline $\begin{array}{l}\text { Forecast } \\
\text { Model }\end{array}$ & \multicolumn{2}{c}{ (baseline) } & \multicolumn{2}{c}{ Naïve } & \multicolumn{2}{c}{ ARIMA 1.1.0 } \\
\hline Group & $1 \mathrm{yr}$ & $2 \mathrm{yr}$ & $1 \mathrm{yr}$ & $2 \mathrm{yr}$ & $1 \mathrm{y}$ & $2 \mathrm{yr}$ \\
\hline Break-even & 2016 & 2022 & 2015 & 2021 & 2015 & 2021 \\
NPV & $€ 28,159$ & $€ 33,264$ & $€ 35,113$ & $€ 38,505$ & $€ 36,599$ & $€ 41,650$ \\
ROI & 2.17 & 1.59 & 2.46 & 1.68 & 2.53 & 1.73 \\
IRR & $8.16 \%$ & $3.68 \%$ & $9.26 \%$ & $4.12 \%$ & $9.46 \%$ & $4.36 \%$
\end{tabular}




\section{The effect of different assumed income tax rates}

In this last sensitivity analysis, the only freely chosen factor, the effective income tax rate, was varied. This analysis was undertaken to test the influence of the income tax factor on final outcomes. In the calculation, the assumed five percent tax rate was first reduced to zero taxes before the outcomes under ten percent tax rate were estimated. According to the calculation, changing the assumed tax rate only marginally altered results as income taxes only accounted for a small share of the overall benefits to the social security system.

Table 33 shows that, without the income tax factor, both types of programs were estimated to need an additional year to break even. For one-year programs, the payback period was increased to 12 years while, for two-year programs, the payback period increased to 18 years. The net present value of one-year programs decreased from $€ 28,159$ to $€ 25,047$, while the ROI slides from 2.17 to 2.04 . The estimated IRR decreased from $8.16 \%$ to $7.35 \%$. The net present value of two-year programs was decreased from $€ 33,264$ to $€ 27,323$, while the ROI decreased from 1.59 to 1.48 . The corresponding IRR of the 25 -year period decreased from $3.68 \%$ to $3.10 \%$.

Under a ten percent income tax rate, the number of years needed for the programs to break even was reduced to 10 years (one-year re-training) and 16 years (two-year re-training). The net present value of one-year programs increased from $€ 28,159$ to $€ 31,271$ while the ROI increased from 2.17 to 2.30 . The estimated IRR increased from $8.16 \%$ to $8.94 \%$. The net present value of two-year programs increased from $€ 33,264$ to $€ 39,206$ while the ROR increased from 1.59 to 1.69 . The corresponding IRR of the 25 -year period increased from $3.68 \%$ to $4.25 \%$.

Table 33: Sensitivity analysis: Income tax rate

\begin{tabular}{lcccccc}
\hline $\begin{array}{l}\text { Assumed } \\
\text { Income Tax Rate }\end{array}$ & \multicolumn{2}{c}{0.00} & 0.05 (baseline) & \multicolumn{2}{c}{0.10} \\
\hline Group & $1 \mathrm{yr}$ & $2 \mathrm{yr}$ & $1 \mathrm{yr}$ & $2 \mathrm{yr}$ & $1 \mathrm{y}$ & $2 \mathrm{yr}$ \\
\hline Break-even & 2017 & 2023 & 2016 & 2022 & 2015 & 2021 \\
NPV & $€ 25,047$ & $€ 27,323$ & $€ 28,159$ & $€ 33,264$ & $€ 31,271$ & $€ 39,206$ \\
ROI & 2.04 & 1,48 & 2.17 & 1.59 & 2.30 & 1.69 \\
IRR & $7.35 \%$ & $3.10 \%$ & $8.16 \%$ & $3.68 \%$ & $8.94 \%$ & $4.25 \%$
\end{tabular}




\subsubsection{Incremental cost-effectiveness of two-year re-training in comparison with one-year re-training}

The focus of this section is on the additional costs and benefits of initial allocation into a, longer, two-year program to determine after what time the allocation into the, more involved programs pays off from a social security perspective. This calculation was performed based on the ATET estimates of two-year participant, which have been estimated in the sub-group analysis in chapter four (cf. section 4.3.3). However, in comparison to the accumulative results retrieved in chapter four, annual results effects were first estimated, before the analysis of the economic costs and benefits was carried out.

\section{Additional costs of longer re-training}

Due to including program dropouts, the associated costs of the re-training programs now slightly differ compared to the previous analysis. Table 34 shows that the cases in which participants were allocated into a partial re-training program on average were dismissed after 9.34 months, while participants allocated a full re-training on average stayed in the program for 21.21 months $^{27}$. Given the program duration, the average program costs per participant and the incremental costs for the two-year program across the three main costs linked to the provision of vocational re-training accruing to public systems were calculated: For partial re-training programs, training costs were, on average, equal to $€ 14,012$ while, for the full re-training programs, training costs of $€ 30,323$ applied.

To cover the basic costs of living, an income replacement benefit (subsistence allowance) of $€ 8,599$ or $€ 21,120$ was paid, over the training period, on average, respectively. These values were retrieved from the primary dataset. If a residential placement was required, additional expenses of $€ 500$ per month accrued. The housing costs, in fact, applied to $9.46 \%$ of all one-year participants and $25.89 \%$ of two-year participants, in the sample that was evaluated. This percentage translates into an average paid housing benefit of $€ 442$ and $€ 2,617$ for the average rehabilitation, respectively.

The overall costs of providing vocational re-training with a proportionate share of people receiving housing benefits, and considering the possibility of program dropouts, equaled $€ 23,052$ for one-year of training and $€ 54,059$ for two-year programs. The incremental costs for a two-year program, per program participant, were, thus, equal to $€ 31,008$.

\footnotetext{
27 The difference in average program duration to the previous section is due to the inclusion of program dropouts.
} 
Table 34: Costs of program participation in one- and two-year re-training and additional program costs of two-year re-training, per program participant

\begin{tabular}{llll}
\hline & $\begin{array}{l}1 \mathrm{yr} \\
\text { re-training }\end{array}$ & $\begin{array}{l}2 \mathrm{yr} \\
\text { re-training }\end{array}$ & 2yr vs. 1yr \\
\hline Program Duration (in months) & 9.34 & 20.21 & +10.87 \\
Accommodation in Boarding School & $9.46 \%$ & $25.89 \%$ & $+16.43 \%$ \\
\hline Training Costs & $€ 14,012$ & $€ 30,323$ & $+€ 16,311$ \\
Income Replacement & $€ 8,599$ & $€ 21,120$ & $+€ 12,521$ \\
Housing Costs & $€ 442$ & $€ 2,617$ & $+€ 2,175$ \\
\hline Total per participant & $€ 23,052$ & $€ 54,059$ & $+€ 31,008$
\end{tabular}

\section{Additional benefits from longer re-training}

In Table 35, the results of the extrapolation process are presented. These results can be considered the predicted overall direct effect of the programs over the rehabilitants' working lives. According to the model estimation, accumulative wage effects over the entire period (including the extrapolation/forecast period) were equal to $€ 64,442$ for two-year programs.

Over the entire evaluation period, two-year program participants were predicted to have 142 additional days on short-term UE benefits, 922 fewer days on long-term UE benefits and 75 more days on social security benefits. While income and social security benefits were forecasted according to the four-year weighted moving average model, the effect on earnings incapacity pension uptake was held fixed after the eight-year observation period, i.e. in the model, a constant effect was assumed.

Table 35: Measured and extrapolated direct effects resulting from program participation in longer re-training

\begin{tabular}{lcc}
\hline Variable & $2006-2013$ & $2006-2030$ (with forecast) \\
\hline Additional Wages & $€ 9,833$ & $€ 64,442$ \\
Short-term UE Benefits & 41 days & 142 days \\
Long-term UE Benefits & -319 days & -922 days \\
Social security Benefits & 115 days & 75 days \\
Earnings Incapacity & $-3.44 \%$ & $-3.44 \%$ \\
\hline
\end{tabular}

Notes: Long-term UE measurement only from 2006-2010, '11-13 was extrapolated. For the two-year intervention group, the 2006 social security estimate was disregarded to prevent double-counting of the income replacement benefit provided (income replacement benefit was part of the direct costs as measured). The effect on earnings incapacity was held fixed. Method of extrapolation: 4-year weighted moving average.

Table 36 provides an overview of the financial benefits to social security of providing a two-year instead of a one-year re-training for participants that participated in a 
two-year re-training (treatment effect on the treated). These comprised additional social security contributions and taxes collected, prevented pay of (short-and long term) UE benefits and other welfare benefits, as well as prevented disability pension payments. The benefits were estimated in comparison to the counterfactual scenario of prior allocation into a one-year program.

The largest benefits to social security were the prevented long-term UE benefits and additional contributions collected, being the result of fewer days on unemployed benefits and increased income of two-year program participants. For two-year retraining participants, long-term contribution collection rose by $€ 26,260$; Income taxes to the federal government rose by $€ 3,222$.

Additionally, compensation payments differed between the different programs. With two-year re-training short-term UE benefits were $€ 3,592$ higher while longterm UE benefits were estimated to be lowered by $€ 18,827$; payments linked to other social security benefits were increased by $€ 1,906$. Additionally, $€ 5,074$ in pension payments were prevented per average participant. Taken together, the overall incremental benefits to the social security system equaled $€ 47,886$.

Table 36: Additional benefits to social security two-year vs. one-year re-training, per participant, 2006-2030

\begin{tabular}{lr}
\hline Benefits to Social Security & 2yr vs. 1yr \\
\hline Additional contributions & $€ 26,260$ \\
Additional taxes & $€ 3,222$ \\
Reduction of short-term UE & $€-3,592$ \\
Reduction of long-term UE & $€ 18,827$ \\
Reduction of social security benefits & $€-1,906$ \\
Reduced earnings incapacity pension & $€ 5,074$ \\
\hline Overall & $€ 47,886$
\end{tabular}

\section{Incremental Cost-Effectiveness}

Table 37 shows that, from the social security perspective, the average net present value of providing two-year re-training opposed to one-year re-training was positive after 18 years in the baseline scenario. The long-run benefits linked to twoyear re-training, after 25 years, were $€ 16,878$ larger than the additional costs of the more involved measures. In other words, for the average rehabilitant that has been allocated into a two-year measure, social security systems received an average return of $€ 16,878$ after 25 years.

Compared to the incremental costs of $€ 31,008$ for providing the extra year of education, this difference equaled an ROI of 1.54. Furthermore, the internal rate of 
return of the projects was calculated in order to evaluate the attractiveness of investing into re-training, irrespective of a presumed social discount rate. The calculated social discount rate, which set the present value of the benefits equal to the costs, equaled $2.93 \%$ for the two-year re-training. On average, 18 years are needed for the additional investments to break-even.

Table 37: Incremental cost-benefit relationship to social security two-year vs. one-year re-training, per participant, 2006-2030

\begin{tabular}{ll}
\hline & $2 \mathrm{yr}$ vs. 1yr \\
\hline Present Value Incremental Benefits & $€ 47,886$ \\
Present Value Incremental Costs & $€ 31,008$ \\
\hline Incremental Net Present Value 25 years & $€ 16,878$ \\
Incremental Internal Social Rate of Return & $2.93 \%$ \\
Incremental Break-even & 18 years (2023) \\
Incremental ROI 25 years & 1.54 \\
\hline
\end{tabular}

Notes: The general approach taken in this comparative analysis was that costs-benefit relationships were considered per allocated rehabilitation case irrespective of rehabilitation outcome.

\subsection{Discussion}

This chapter has provided an empirical example evaluation of the costs and benefits of vocational re-training for social security providers. Initially, a literature survey, identified the main costs to social security. In addition, the benefits calculated in the quasi-experiments in chapters three and four were re-estimated on an annual basis before being extrapolated to project the development of employment outcomes outside the observation period. Next, the primary effects were monetarized and compared with the incurred costs. The results of the economic analysis were threefold: First, it has been shown that both types of re-training measures were, on average, cost-effective from the social security perspective. However, the evaluated measures payed off only in the very long run. This challenge the recent state-of-theart in program evaluation usually appraising only one, two or three years of followup. A second major result from this analysis was that the financial payback period of partial re-training was shorter in comparison to the longer full-retraining measures. Third, for the participants that were allocated a two-year program, the incremental effects to social security were positive, however, the payback period of the additional investment was substantial.

In particular, the long-run benefits linked to one-year re-training were $€ 28,159$ larger than the costs of providing the measure. For two-year re-training, the returns were $€ 33,264$ larger in comparison with program costs. The corresponding ROI ratios (benefits over costs) equaled 2.17 and 1.59, respectively. These results mean that, for 
every euro spent on partial re-training, the social security system received an average return of $€ 2.17$, in the form of additional taxes and contribution generated and compensation pay prevented, in the long run. For every euro spent on full retraining, the social security system received an average return of $€ 1.59$, in the long run. The calculated social discount rate, which set the present value of the benefits equal to the costs, equaled $8.16 \%$ for the one-year re-training programs and $3.68 \%$ for two-year re-training programs. Payback periods were assessed to be equal to 11 years (one-year re-training) and 17 years (two-year re-training).

In a sensitivity analysis, it was further analyzed to what extent the cost-effectiveness changes as a result of altering some of the crucial assumptions to the model applied. These changes included introducing a fixed social discount rate, to simulate the effects considering stronger intertemporal time preferences for the present; changing the forecasting model to a naïve and a first-order autoregressive model and altering the assumed income tax rate due to it being the only assumed (freely chosen) variable in the calculation.

While, under a two percent social discount rate, both programs were still costeffective when evaluated over the 25-year period, with five percent discounting, the, more involved, two-year programs were no longer cost-effective. With regards to the forecasting model used, both naïve and autoregressive modeling of future benefits yielded higher returns to the social security system compared to the fouryear moving average used in the main estimation. Thus, the applied main forecasting method can be considered most conservative of the three models tested. Changing the assumed income tax rate, as tested in the last part of the sensitivity analysis, only marginally affected the final results.

In a different analysis, it has been shown that program allocation into a two-year as opposed to allocation into a one-year re-training measure broke even after 18 years (average treatment effect on the treated). The long-run benefits linked to two-year re-training after 25 years were $€ 16,878$ larger than the additional costs of the more involved measures. In other words, for the average rehabilitant who has been allocated into a (longer) two-year measure, social security systems receive, on average, a positive return in the long run. Compared to the average incremental costs of $€ 31,008$ for providing the longer duration re-training measures, this increase equaled an ROI of 1.54. Furthermore, the internal rate of return of the additional investment was calculated to evaluate the attractiveness of investing into the longer re-training measure as opposed to providing only one-year re-training for the given sample of two-year participants. The calculated social discount rate, which set the 
present value of the incremental benefits equal to the additional costs of providing two-year re-training (instead of one-year re-training), equaled $2.93 \%$.

The results of the foregone analysis suggest that policymakers have a financial incentive to invest in both types of vocational re-training programs as they were cost-effective in the long run. Given that the produced findings were calculated based on average values, the results from this analysis should, however, be treated with caution since allocation into vocational rehabilitation and consequent outcomes are dependent on the individual situation of the rehabilitants, as shown previously in this thesis.

Further work needs to be carried out on the influence of currently non-quantifiable benefits such as the health benefits from working or the spillover effects to other household members. Additionally, it was not possible to observe or measure the motivation and abilities of the rehabilitants, which may lead to imprecise estimates. Furthermore, estimating some input parameters for which no empirical information could be found should be substantiated by future research.

Despite these potential limitations, this chapter offers comprehensive results to better understand the economic effects of rehabilitation measures, contributing substantially to the understanding of the economic consequences of vocational retraining programs. Given that the actual financial costs of re-training for the whole population accumulate to several hundred million euros, the foregone evaluation puts the returns to re-training in relation, showing that the measures pay off, also from the social security perspective.

Based on the cost-benefit relationships estimated in this chapter, and given some uncertainty about the long term, a possible explanation for the current expansion and political prioritization of the shorter one-year programs is offered. Policymakers and the individual's interest may conflict with each other in some circumstances if the individual might prefer the, more involved re-training but for cost-efficiency reasons is only allocated a one-year program. 

6. Conclusions and policy implications 
This final chapter summarizes the main contributions that this thesis has made and provides a summary of the research findings. Next, the policy implications for vocational re-training in Germany as well as for vocational rehabilitation programs, in general, are discussed. The chapter continues with a discussion of the limitation of this study and proposes areas for future research.

\subsection{Contributions of this thesis}

Work disability is a challenge for social security systems, affecting millions of people worldwide. At the same time, social policymakers have implemented vocational rehabilitation programs aimed at improving the chances of re-employment despite physical limitations and other health restrictions. So far, much of the empirical evidence on the effects of vocational rehabilitation in Germany has been confined to small sample sizes and specific institutional settings. In this thesis, unique administrative data, a relevant time of follow-up and advanced statistical measures to handle confounding have been utilized in order to provide new and informative evidence on the effects of vocational rehabilitation on employment outcomes in Germany.

This thesis has made four major contributions to the analysis of the effectiveness of vocational rehabilitation programs for persons with disabilities.

First, the research investigated how socio-economic factors influenced the employment outcomes after participation in vocational rehabilitation. The analysis included identifying which factors positively affect re-employment chances and which socio-economic characteristics tend to act as barriers to successful work reintegration. Several risk factors were consequently identified that were associated with little improvement in the employment status: Among the different factors tested, old-age, low prior educational outcomes and irregular employment relationships were especially associated with less improvement in the employment status. The attained findings in this chapter support the argument that providers of vocational rehabilitation services need to be aware of the influence demographicand occupational factors have on re-employment chances: the socio-economic status and the work environment significantly affect return-to-work outcomes after participation in vocational rehabilitation.

Second, while taking the effect of confounding variables into account, the analyses examined to what extent the vocational re-training program, the most time-intensive and costly type of vocational program available, impacted employment outcomes and to what extent the programs reduced the likelihood of receiving an earnings incapacity pension. The estimated treatment effects provide policymakers with detailed information about the interventions' abilities to improve the individuals' 
employment statuses and show to what extent these improvements were sustained over-time: The result of this analysis was that, despite the presence of physical limitations, completing the re-training measures greatly improved the employment situation of the rehabilitants, i.e. the measures resulted in more days in employment and higher annual income earned while at the same time dependency on social security benefits was reduced. In addition to its empirical relevance, the research also contributes to the methodological debate on how to estimate the impact of labor market programs on employment outcomes from observational data using quasiexperiments. This approach, so far, has been widely underutilized in vocational rehabilitation research.

Third, the effects of two-year and one-year vocational re-training programs were directly compared to provide a better understanding of the duration effects of the re-training measures. The results showed that the disadvantages of longer program duration were offset by significantly higher employment outcomes in the long term for participants of two-year re-training programs. From the perspective of the retraining participants, the additional year of re-training generally paid off, i.e. the treatment effects on the treated with regards to income and employment days were positive for two-year re-training participants and the likelihood to be awarded a pension due to a reduced earnings capacity was also lower. The large reduction in days with long-term unemployment benefits indicates that full re-training programs had a significantly greater impact with regards to preventing permanent exit from the labor market. Whereas the increase in days with other social security-benefits was mainly due to the longer program duration, the shift in benefit recipience may be advantageous, as long-term unemployment seems to have been reduced substantially.

Finally, the cost-effectiveness and efficiency of re-training were evaluated from a social security providers' perspective. While chapters three and four showed what impact vocational rehabilitation may have on the individuals involved; in the fifth chapter, secondary effects for social security were calculated. These comprise increased contributions and tax collection and a reduction in the paid compensation benefits. Relating the benefits to the costs of providing the programs, the estimated financial balance sheets illustrate the large economic potential of meaningful vocational rehabilitation, showing whether the programs were cost-effective.

Taken together, this thesis adds to a growing body of literature on the effects of labor market activation programs for people with a disability. While re-affirming the important role of micro-level determinants, this thesis has provided an extensive example of the individual and fiscal effects that are possible through meaningful 
vocational rehabilitation measures. Re-training has been found to have a large impact on employment participation and on livelihoods. By enabling people with a disability to return to work, vocational rehabilitation programs contribute to reducing the economic disadvantages they face and facilitate their social (re-) integration.

\subsection{Summary of main findings}

The following section summarizes the main findings in order to answer the four research questions posed in the introduction section of this thesis:

1. To what extent do socio-economic factors influence return-to-work outcomes after participation in vocational rehabilitation? (Chapter 2)

In the second chapter, employment outcomes and their determinants after participation in vocational rehabilitation have been investigated through an analysis of a representative panel dataset of German rehabilitants. The focus on patient-level characteristics has only received limited attention in research as microdata in the present form is rare. As such, this chapter provides nationally and internationally new and relevant insights with regards to the effects of demographic factors and job characteristics on employment outcomes for vocational rehabilitants.

At first, through a comparison of income and employment data over several years before and after program entry, cohort and individual employment results were measured. Next, it was examined to what extent differences in the return-to-work outcomes among rehabilitants were linked to their socio-economic status. The factor analysis showed that before-after differences in the employment outcomes can, to a substantial extent, be predicted based on the participants' socio-demographic and employment characteristics. Various socio-economic characteristics seemed to facilitate employment reintegration while others acted as a barrier.

Among the different factors tested, the personal employment and income history was the strongest predictor of future labor market outcomes. Moreover, it has been shown that the patients' ages, educations, jobs, and employment types had a large and significant effect on employment outcomes after vocational rehabilitation. The findings are in line with previous findings in the field (see the review by Streibelt \& Egner, 2013), which also found a strong effect of various individual factors on return to work outcomes (such as income before admission, the rehabilitants age and the employment or occupational status).

Younger, better educated, white-collar workers and those employed in full-time contracts exhibited significantly greater changes in employment outcomes than their 
respective comparison groups. Differences in employment outcomes with regards to gender were, in comparison, small, but also significant. To provide more detailed results with regards to gender-specific effects, a stratified regression model was further added to the analysis. The stratified model showed gender-specific effects of socio-economic factors. Most notably, the coefficients for being widowed, divorced and unemployed changed signs.

The estimated differences throughout the models tested persisted, also after controlling for intra-group heterogeneity in employment history. The relatively good fit of the change score regression models in comparison to a similar study based on medical rehabilitation data from the same data source (Petrunyk, et al., 2015) can, to some extent, be linked to the more cohesive data and a longer time horizon used. Another explanation is that employment results after vocational rehabilitation are more dependent on individual characteristics compared to employment outcomes after medical rehabilitation.

According to the analysis, the socio-economic status plays a substantial role in an individual's return to work. The measured differences in employment outcomes after participation in vocational rehabilitation were closely related to the participants' socio-demographic and employment characteristics. The attained findings in this analysis support the argument that providers of vocational rehabilitation services need to be aware of the influence demographic and occupational factors have on employment outcomes in the return to work of people with a disability.

2. To what extent do vocational re-training programs impact employment outcomes and influence dependency on social security benefits? (Chapter 3)

In the third chapter, an empirical example of how to use administrative panel data when evaluating re-training programs for people with disabilities has been provided. In the absence of randomized experiments, using an applicant-based comparison group was the most appropriate option to evaluate treatment effects. According to the estimates in Chapter 3, participation in vocational re-training was associated with significant improvements in the employment status while also reducing dependency on social security benefits and the likelihood of an earnings incapacity pension.

Based on a random sample of 2,399 individuals admitted into vocational re-training in the year 2005, the impact of vocational re-training was examined by estimating the average treatment effect and the average treatment effect on the treated in relation to the counterfactual case of no completed re-training. The analyzed 
outcomes comprised changes in individual earnings and days with employment, earnings incapacity pension receipt and changes in the number of days on social security and unemployment benefits.

The results of this analysis suggest that both types of vocational re-training programs analyzed significantly improved the income and employment situation of the individuals involved over the first eight years after program admission. After other factors had been controlled for, graduates of one-year re-training were, on average, employed for an additional 405 days, 95\% CI [249 days, 561 days], and had earned $€ 24,260$ more than without re-training, 95\% CI [€12,805, €35,715]. Two-year program completers, on average, were employed for 441 additional days, $95 \% \mathrm{CI}$ [349 days, 534 days], and had earned $€ 35,972$ more than without completed retraining, 95\% CI [€27,743, €44,202].

Moreover, graduating from a re-training program significantly reduced the number of days on social security benefits and influenced the number of days on unemployment benefits. In addition, the measures also reduced the likelihood of receiving an earnings incapacity pension in comparison to the counterfactual scenario of no re-training. Over the eight-year observation period, one-year program graduates had a $3.1 \%$ lower likelihood of being awarded a pension due to a reduced earnings capacity, 95\% CI [-7.8\%, 1.6\%]; two-year program graduates had a $6.0 \%$ lower likelihood, $95 \%$ CI [-9.2\%, $-2.9 \%]$.

Further work needs to be carried out to determine under which conditions returnto-work outcomes could be improved by providing individuals with a two-year instead of a one-year re-training. The estimated mean employment effects were generally larger for two-year re-training programs in comparison to one-year programs, indicating that some individuals could have potentially benefitted from allocation into a full re-training program. However, the response to treatment is likely to be heterogeneous and dependent on the individual situation and preferences of the rehabilitants. In order to better understand the conditions under which allocation into a more involved program could lead to better outcomes, more information on the complex relationship between the applicant and the provider in the application and screening process is needed.

The choice of method for the analysis of absolute treatment effects in Chapter 3 adds to the methodological debate on how to estimate program effects from observational data for public policymaking. The main point of debate is whether policymakers can confidently rely on treatment effect estimates obtained from quasi-experimental research settings. Much of the literature indicates that the most common weakness in the quasi-experimental research setting is linked to using an external comparison 
group. While different training programs exhibit different institutional settings, the foregoing analysis suggests that it is possible, at least for the case of vocational rehabilitation in Germany, to make use of information on program dropouts as an appropriate internal control group to obtain meaningful treatment effect estimates.

One needs to be careful when drawing causal conclusions from the estimated relationships. Many of the persons in the group of program dropouts actually participated to some extent in the re-training measures before leaving the program, thus, also profiting from the skill training, while at the same time, using up time that could have been spend otherwise if they would have never participated in the measures at all (e.g. to earn money or to get a different training). It remains a topic of discussion, whether a person that participates in a re-training but never graduates from it benefits more from the measure in relation to the time that is lost. On the one hand, failed examination and other performance issues being the main non-medical dropout reasons point to lower abilities of the group of program dropouts which would mean that the effects were overestimated; the group of program dropouts carried some unmeasured confounders that negatively affected their performance (negative dropout). On the other hand, the argument can be brought forward, that some of the program dropouts likely discontinued their training because they found another job (perhaps even linked to the benefits received from re-training). This would mean that the estimated relationships were underestimated, since those that were offered employment and leave the program carried some unmeasured confounders that positively affected their earnings ability (positive dropout). Accordingly, the set-up of the quasi-experiment should be kept in mind when drawing conclusions from the estimated relationships.

Despite these potential limitations, the treatment effects presented in this study are an important contribution to the empirical knowledge on the influence vocational re-training on the employment re-integration of individuals with disabilities in Germany. This claim is based on the availability of a unique database and the application of appropriate statistical methods, which have allowed us to evaluate the impact of the re-training programs. According to the estimated treatment effects in this chapter, completing a vocational re-training was associated with significant improvements in the employment status while also reducing dependency on social security benefits. Policies to promote the labor market re-integration should consider that vocational re-training may be an effective tool for sustainably improving the employment situation of people with a disability. 
3. Are there significant differences between the effects of one- and two-year re-training programs with regards to income earned, employment days and social security benefit receipt? (Chapter 4 )

In the fourth chapter, it has been shown how longitudinal administrative data in the area of publicly funded training programs can be used to measure the differential impact of training programs on labor market outcomes. The main research goals were to examine the extent to which the two most commonly allocated types of measures, further training, and re-training, exhibit similar employment effects. Those effects included changes in individual earnings developments, days with employment and days with unemployment benefits.

The descriptive statistics section of this chapter showed that the sampled participants of further training measures were older, more often unemployed at program admission and disproportionally more often live under unfavorable labor market conditions compared to re-training participants. However, there was also substantial overlap with regards to many of the measured covariates, which was a key prerequisite in order to carry out the impact analysis. Using the inverse probability-weighted regression adjustment method, the covariates were first balanced across comparison groups in order to establish comparability, before the earnings differentials were estimated.

By prolonging the follow-up in comparison to previous research, the more involved, two-year re-training programs were found to be more effective regarding long-term work participation outcomes as one-year vocational re-training programs. Although two-year re-training measures require an additional year of education, the average treatment effect over the first eight years after program admission was positive; i.e. participation in two-year re-training was associated with more income compared to participation in one-year re-training only, despite an additional year of program duration.

Over the first eight years after program admission, the average treatment effect of two-year re-training compared to one-year re-training, for the whole study population, equaled $€ 10,126,95 \% \mathrm{CI}[€-1,327, € 21,578]$. Furthermore, participation in two-year re-training was associated with more days in employment, fewer days on long-term unemployment benefits and a reduced risk for an earnings incapacity pension. On the other hand, participation in one-year re-training was associated with significantly fewer days on social security benefits and on short-term unemployment benefits. 
Since not all individuals can be considered eligible for the longer duration measures, separate analyses of the ATET for the comparison groups were carried out. The subgroup analysis showed, to which extent the two-year participants benefitted from actual program participation in the longer type programs and to which extent the one-year group would have benefitted from the longer duration programs.

The first result of the sub-group analysis was, that, over the eight-year observation period, participation in a two-year vocational re-training program was associated with an $€ 11,270$ higher income, $95 \% \mathrm{CI}[€-761, € 23,300]$, compared to the income achieved after hypothetically having been allocated into a one-year re-training program instead. The increase in income was a consequence of more accumulative days in employment after eight years despite longer program duration and higher dropout rates. The duration of transfer payments, on the other hand, was characterized by shifts, i.e. being allocated a two-year re-training was linked to fewer days with long-term unemployment benefits but more days with short-term unemployment benefits and more days with other social security benefits. Participants of two-year programs had 211 fewer days with long term unemployment benefits, 95\% CI [-296 days, -125 days], but 41 additional days with short term unemployment benefits, 95\% CI [18 days, 65 days], and 238 additional days with other social security benefits, 95\% CI [190 days, 286 days]. Whereas the increase in days with other social security-benefits was mainly due to the longer program duration, the shift in benefit recipience may have been advantageous, as long-term unemployment seems to have been reduced substantially. In addition, two-year re-training participants had a 3.4\% reduced risk for a pension due to a reduced earnings capacity, $95 \%$ CI $[-6.9 \%, 0.0 \%]$. The estimate was, however, not signficant at the five percent level

The second result of the sub-group analysis was, that, over the eight-year observation period, participation in a one-year vocational re-training program was associated with a $€-3,557$ lower income compared to the income achieved after hypothetically having been allocated into a two-year re-training program instead, $95 \%$ CI $[€-12,630, € 5,516]$. Neither the effect on income nor the effect on employment days were statistically significant at the five percent level. In fact, the standard error of the estimate of employment days was substantial indicating that the employment effects were very heterogeneous. With regards social-security and welfare benefit receipt, participants of one-year programs had 164 more days on long-term unemployment benefits, 95\% CI [ 93 days, 235 days], but 19 fewer days on short-term unemployment benefits, $95 \%$ CI [-43 days, 5 days], and 264 fewer days on other social security benefits, $95 \%$ CI [-302 days, -226 days]. This was in comparison to having been allocated into the longer re-training programs instead. The estimated 
differences in the number of days with social security and long-term unemployment benefits were significant at the one percent level. The effect on days with short-term unemployment benefits was not statistically significant at the five percent level. Moreover, the small increase in the likelihood to receive an earnings capacity pension, of $0.9 \%$, was also not significant, $95 \%$ CI [-2.4\%, $4.2 \%]$.

The results indicate, that program allocation was to some extent effective. The ATET of two-year program participants was larger compared than both the ATE and the ATET of one-year program participants (the latter reflects how much lower the income of the one-year participants was compared to having participated in a twoyear program instead). Based on the calculations, the average participant that was allocated into two-year re-training benefited more from the measures, than the average participant that was allocated a one-year re-training would have had. While the retrieved estimates are based on averages, further explorative analysis is needed to examine the conditions under which participants of one-year programs could profit from increased duration of re-training. The response to treatment seems to be heterogeneous and dependent on the individual situation and preferences of the rehabilitants.

Further work is needed to determine under which conditions return-to-work outcomes could be improved by providing individuals with a two-year instead of a one-year re-training. The estimated mean employment effects were generally larger for two-year re-training programs in comparison to one-year programs, indicating that some individuals could have potentially benefitted from allocation into a full re-training program. However, the response to treatment is likely to be heterogeneous and dependent on the individual situation and preferences of the rehabilitants. One-year program participants seem to be a more marginalized group, as such, there might be specific reasons to support them with less intensive measures.

Improving the understanding of the conditions under which allocation into a more involved program could lead to better outcomes requires more information on the complex relationship between the applicant and the provider in the application and screening process. Program participation depends both on rehabilitant eligibility and on the selection by the person in charge or self-selection by potential participants. Thus, it would be relevant to further explore the perspective of the individual before and during the period of rehabilitation as well as the role of the provider in programmatic decision making. 
4. What are the financial returns for providers of the re-training programs: are the measures cost-effective? (Chapter 5)

The fifth chapter provides an empirical example evaluation of the costs and benefits of vocational re-training for social security providers. The main costs of the measures were identified through a literature survey. Benefits from the quasi-experiments in chapters three and four were extrapolated in order to project the development of outcomes outside the observation period. The estimates from chapter three were used to evaluate the absolute cost-effectiveness of the two types of re-training programs in comparison to the do-nothing scenario. The estimates from chapter four were used to evaluate the incremental cost-effectiveness of the allocation into the more involved two-year re-training measures in comparison to allocation into the, less involved, one-year re-training measures.

The results of the analysis were twofold.

The first result was that both types of re-training measures evaluated were, on average, cost-effective from the social security perspective when compared against the do-nothing alternative. Despite the restrictive assumptions used, the estimated balance sheets demonstrate the large economic potential of investing in work reintegration measures. In the baseline scenario, one-year measures broke even after 11 years, while two-year measures broke even after 17 years. After 25 years, the estimated benefits linked to one-year re-training were $€ 28,159$ larger than the costs of providing the measure. For two-year re-training, the returns were $€ 33,264$ larger in comparison with program costs. The corresponding ROI ratios (benefits over costs) for one- and two-year programs over the 25-year evaluation period were equal to 2.17 and 1.59 , respectively. This means that, for every euro that was spent on the one-year re-training, the public received an average return of $€ 2.17$ in the form of additional taxes and contribution generated and compensation pay prevented, in the long run. For every euro spent on two-year re-training, the public received an average return of $€ 1.59$. Furthermore, the internal rate of return of the projects was calculated, to evaluate the attractiveness of investing into re-training, irrespective of a presumed social discount rate. The calculated social discount rate, that set the present value of the (inflation-adjusted) benefits equal to the costs, equaled $8.16 \%$ for one-year re-training programs and $3.68 \%$ for the two-year re-training.

In a sensitivity analysis, it was further analyzed to what extent the cost-effectiveness of the two program types changes as a result of altering some of the crucial assumptions to the applied model. This analysis included introducing a social discount rate to simulate the effects considering stronger intertemporal time preferences for the present, changing the forecasting model to a naïve and a first- 
order autoregressive model and altering the assumed income tax rate due to it being the only assumed (freely chosen) variable in the calculation.

While, under a two-percent social discount rate, both programs were still costeffective when evaluated over the 25-year period, with five percent discounting, the, more involved, two-year programs were no longer cost-effective. With regards to the forecasting model used, both naïve and autoregressive modeling of future benefits yielded higher returns to the social security system compared to the fouryear moving average used in the main estimation. Thus, the applied main forecasting method can be considered the most conservative of the three models tested. Changing the assumed income tax rate, as tested in the last part of the sensitivity analysis, only had a marginal effect on results.

The results of the first cost-effectiveness analysis suggest that policymakers have a financial incentive to invest in both types of vocational re-training programs as they were found to be cost-effective in the long run. Given that the produced findings are based on average values, the results from this analysis should, however, be treated with caution. Allocation to vocational rehabilitation and consequent outcomes are largely dependent on the rehabilitants' individual situations, as shown previously in this thesis. The estimated payback periods of the programs were rather long, making the factor age, next to the prior earnings level, likely to be to most decisive factor when wanting to draw further conclusions about the cost-effectiveness on a case by case basis. Of course, economic considerations may not be the only factor in the decision-making process.

Moreover, based on the cost-benefit relationships estimated in the first costeffectiveness analysis, and given some uncertainty about the long term, a possible explanation for the current expansion and political prioritization of the shorter oneyear programs is offered: the break-even point of one-year programs was attained much earlier (11 instead of 17 years) and the ROI and IRR wee much larger. Policymakers and the individual's interest may, thus, conflict in some circumstances as the individual might prefer the, more involved, two-year measure, but is only allocated, a less costly, one-year measure. While chapters three and four showed that, for the individuals involved, two-year programs, on average, had a larger impact on employment outcomes, the results of this analysis might explain why, from the social security perspective, one-year programs are increasingly often assigned.

The second results were that while the payback period of the longer duration measures was much longer and the ROI and IRR much smaller, the net present value over the entire 25-years was in fact larger for the, more involved, two-year measures 
compared to one-year measures. In addition, a second analysis on the incremental effects of two-year re-training compared to one-year re-training showed, that for the average rehabilitant, that had been allocated a, more costly two-year measure, the additional financial investments made broke even after 18 years. Over the entire 25year simulation period, long-run benefits linked to two-year re-training, were $€ 16,878$ larger than the additional costs of the more involved measures. With incremental costs of $€ 31,008$ for providing the extra year of education, this equaled an ROI of 1.54 over the twenty-five-year evaluation period. Furthermore, the internal rate of return of the additional investment was calculated. The calculated social discount rate that set the present value of the benefits equal to the additional costs of providing two-year re-training (instead of one-year re-training) equaled $2.93 \%$.

These estimates need to be treated with caution, since it can be anticipated that the outcomes are heterogeneous. Some people may be more suitable to participate in full re-training measures while others may favor shorter measures. Although this analysis incorporated various proxies to model individual characteristics such as past earnings ability or the education level, many soft factors that may influence treatment decisions cannot be modeled with the data at hand. Furthermore, the estimates provided above reflect the treatment effects for those that received the longer-duration training. The potential treatment effects for those that have been allocated a one-year re-training were predicted to be much smaller.

Taken together, chapter 5 has provided a comprehensive analysis of the secondary effects of vocational rehabilitation contributing to a better understanding of the economic consequences of applied rehabilitation measures. Both types of re-training measures evaluated were, on average, cost-effective from the social security perspective. Given that the actual financial costs of re-training for the whole population accumulate to several hundred million euros, the foregone evaluation shows what potential returns such measures can bring to public systems.

\subsection{Policy recommendations}

The German vocational re-training program for people with a disability has been found to have a positive impact on rehabilitants' employment outcomes. The results showed that the principal objective, namely, to improve participation in employment, was generally achieved. Contrary to the common misconception that "off-the-job training" is relatively ineffective, this thesis has provided an empirical example of the positive impact of the programs. Therefore, the first policy recommendation offered by this thesis is to continue to invest in vocational retraining as it generates a positive impact for the participating individuals. 
Another conclusion from the analysis in chapter three is that program dropout was associated with significantly worse work participation outcomes, compared to program graduates; unsuccessful completing seems to be a strong predictor for worse employment outcomes. Accordingly, preventing dropout and, thus, improving the overall effectiveness and efficiency of re-training measures is another important policy target. Since about half of the dropouts were related to medical reasons, better a priori screening and better medical supervision during the programs may contribute to reducing program dropout rates. Regarding the nonmedical dropouts, many were due to performance issues and failed examinations. Further investigation is needed into why programs that were deemed to be appropriate turned out to be infeasible for certain individuals.

The literature on the comparative effects of one- and two-year re-training has previously found that reduced training may lead to better outcomes. A shortening of the average duration of measures tends to have a favorable effect in the short term because the lock-in effect loses weight compared to the program effect. However, when assessed over longer follow-up, the more involved training programs have been found to be more effective since the accumulative employment effects were larger (although not significant). From the perspective of two-year re-training participants, the additional year of re-training generally paid off, i.e. the treatment effects on the treated with regards to income and employment days were positive for two-year re-training participants and the likelihood to be awarded a pension due to a reduced earnings capacity was also lower. These results are an important reassurance, that the additional time invested into the longer training measure actually paid off for the individuals involved.

When separating the analysis into the sub-samples of participating individuals in the one- and two-year re-training, respectively, the results indicate that program allocation was to some extent effective. The average treatment effect on the treated of two-year program participants was larger compared to both the sample average treatment effect across both treatment groups and the average treatment effect on the treated of the one-year program participants (which shows the income loss associated with reduced duration training). Thus, the average participant that was allocated into two-year re-training benefited more from the measures, than the average participant that was allocated a one-year re-training would have. While these estimates are based on averages, further explorative analysis is needed to examine the conditions under which participants of one-year programs could profit from an increased duration of re-training. 
The performed analysis in chapter two showed how the individuals' socio-economic statuses strongly influenced employment outcomes after participation in vocational rehabilitation. This, rather obvious, finding has rarely been substantiated by empirical evidence, let alone considered in the assignment practice. This study has shown that factors such as greater age or low prior educational attainments decrease the chances of a successful return to employment. To improve return-to-work outcomes, it is therefore recommended that policy targets these groups. For example, by improving access to and the effectiveness of individual case management.

Vocational re-training was cost-effective when compared to the alternative of no completion of re-training since it increases wages and employment days while reducing the number of days on social security and unemployment benefits. Accordingly, policymakers should make use of the provided empirical evidence to strengthen the role of vocational re-training with regards to its usage and application within the overall social protection system - vocational rehabilitation pays off, also from the social security perspective.

\subsection{Limitations and areas for future research}

The limitations of this thesis have been discussed in each chapter and are summarized below together with propositions for future research.

The results of the second chapter show that an avenue for future studies would be research into the specific barriers to employment of the most vulnerable socioeconomic groups of rehabilitants, i.e. older people, those with little prior education or those in previously irregular employment relationships. The results point to potential problems in the screening mechanisms that should be reconsidered, potential problems during the period of treatment as well as general reintegration problems, which could stem from discrimination in the labor market, or from incentives and disincentives provided by the retirement and unemployment benefit system. Explorative studies are needed to examine in more detail the underlying factors of these poor return-to-work outcomes since the data that was used in this analysis is not suitable to answer these questions.

The relationship between the duration or level of schooling and earnings estimated in chapters three and four do not necessarily imply causality. Moreover, the range of potential variables for calculating the propensity score was limited. For example, it was not possible to observe or measure the rehabilitants' motivation or other soft factors that have an influence on employment outcomes, which may lead to imprecise estimates. Incorporating proxies for these "factors" into future analysis could contribute to a better understanding of individual education and work 
reintegration trajectories. Another interesting area for future research could be to address potential measurement error in the treatment variables, using instrumental variables to establish causality. In the absence of randomized control trials, policy changes could be a source of promising instruments since they affect the allocation to treatment independently of preferences. Other, possible instruments include the distance to the nearest training facilities or the parental education level. ${ }^{28}$

A potential drawback of the sample used was the comparably small number of controls in relation to the number of treated subjects in the treatment effect analysis. However, the analysis of standardized differences has shown, that despite the relatively small number of controls, effective re-weighting was carried out, which limited the differences in covariates between comparison groups to a negligible level. Additionally, the majority of estimated treatment effects were statistically significant signaling that the number of controls was sufficient to detect differences in outcomes. A potential area for future research could be to include several cohorts of rehabilitants into the analysis to establish a larger sample size.

While about half of program dropouts left the program for medical reasons, further explorative analysis is needed to find out, whether assistance provided during the period of rehabilitation could be improved (in order to prevent the occurrence of medically related dropouts). This includes knowing more about the barriers with regards to the access to the re-training measure, which would allow a more accurate prediction of when re-training is medically appropriate and what type of additional assistance (accommodation) is needed for the measures to have the greatest chance of success. In addition, further analysis could investigate whether program dropout could have been predicted before the start of the measures based on better screening mechanisms and knowledge about the program contents.

Apart from medical reasons for program dropout, failed examinations and other performance issues were the other main reasons why individuals were not able to finish a re-training program. Consequently, future research should further investigate possible assistance that can be provided, during the period of rehabilitation, to increase graduation rates. Moreover, the outcome of allocation practices should be further examined: Are the individuals satisfied with the occupational re-training choice? Would they rather have enrolled in a different trade or occupation? Are there other reasons that influence how well the rehabilitants

\footnotetext{
28 The distance to a training facility is generally considered to be one of the most important non-monetary barriers impeding access to education programs. On the other hand, parental education instruments build on the concept of inter-generational correlation of education outcomes.
} 
perform in the examinations? Given the large benefits associated with completed retraining and the comparatively large costs of these measures, these are relevant questions.

Further work must be undertaken to determine under which conditions return-towork outcomes could be improved by providing individuals with a two-year instead of a one-year re-training. The estimated mean employment effects were generally larger for two-year re-training programs in comparison to one-year programs, indicating that some individuals could have potentially benefitted from allocation into a full re-training program. However, the response to treatment is likely to be heterogeneous and dependent on the rehabilitants' individual situations and preferences.

To better understand the conditions under which allocation into a more involved program could lead to better outcomes, more information is needed on the complex relationship between the applicant and the provider in the application and screening process. Program participation depends both on the eligibility of the applicant and on the selection by the person in charge or self-selection by potential participants. Thus, it would be relevant to investigate through further explorative studies the individual perspective before and during the period of rehabilitation as well as the provider's role in programmatic decision making. 


\section{List of references}

§32a EStG. German Income Tax Act of 8 October 2009 (BGBl. I S. 3366, 3862 (Federal Law Gazette I, $p .3366,3863))$, which has most recently been amended by Article 1 of the law from of 4 August 2019 (BGBl. I S. 1122 (Federal Law Gazette I, p. 1122).

Aakvik, A. (2003). Estimating the employment effects of education for disabled workers in Norway. Empirical Economics, 28(3), 515-533.

Adams, J. H. ., \& Williams, A. C. D. C. . (2003). What affects return to work for graduates of a pain management program with chronic upper limb pain? Journal of Occupational Rehabilitation, 13(2), 91-106.

Allison, P. D. (1990). Change Scores as Dependent Variables in Regression Analysis. Sociological Methodology, 20, 93.

Angrist, J., \& Hahn, J. (2004). When to control for covariates? Panel asymptotics for estimates of treatment effects. Review of Economics and Statistics, 86(1), 58-72.

Austin, P. C. (2011). An introduction to propensity score methods for reducing the effects of confounding in observational studies. Multivariate Behavioral Research, 46(3), 399-424.

Baril, R., Berthelette, D., \& Massicotte, P. (2003). Early return to work of injured workers: multidimensional patterns of individual and organizational factors. Safety Science, 41(4), 277-300.

Beiler, J. (2013). Employability as a problem of the vocational rehabilitation of the disabled. Rehabilitation (Germany), 52(5), 352-356.

Berecki-Gisolf, J., Clay, F. J., Collie, A., \& McClure, R. J. (2012). The Impact of Aging on Work Disability and Return to Work. Journal of Occupational and Environmental Medicine, 54(3), 318-327.

Bethge, M., \& Streibelt, M. (2015). Are Two-Year Vocational Retraining Programs Really Better than One-Year Programs? Findings of a Propensity Score Matched Analysis. Die Rehabilitation, 54(6), 389-395.

Biewen, M., Fitzenberger, B., Osikominu, A., Völter, R., \& Waller, M. (2006). Beschäftigungseffekte ausgewählter Maßnahmen der beruflichen Weiterbildung in Deutschland: Eine Bestandsaufnahme. Zeitschrift Für Arbeitsmarktforschung, 4, 365-390.

Biewen, M., Fitzenberger, B., Osikominu, A., \& Waller, M. (2007). Which Program for Whom? Evidence on the Comparative Effectiveness of Public Sponsored 
Training Programs in Germany. IZA Discussion Papers Series, 2885(07).

Bitton, A., Onega, T., Tosteson, A. N. A., \& Haas, J. S. (2014). Toward a better understanding of patient-reported outcomes in clinical practice. American Journal of Managed Care, 20(4), 281-283.

Blackwell, T. L., Leierer, S. J., Haupt, S. S., Kampitsis, A., \& Wolfson, J. R. (2004). Prediction of Vocational Outcomes for Workers' Compensation Claimants with Back Injury. Journal of Applied Rehabilitation Counseling, 35(2), 32-38.

Bläsche, A., Brandherm, R., Eckhardt, C., Käpplinger, B., Knuth, M., Kruppe, T., ... Schütt, P. (2017). Qualitätsoffensive strukturierte Weiterbildung in Deutschland. Düsseldorf: Hans-Böckler-Stiftung.

Bliss, C. I. (1934). The method of probits. Science, 79(2037), 38-39.

Bundesagentur für Arbeit. (2006). Arbeitsmarkt 2005. Nürnberg: Bundesagentur für Arbeit.

Burstedde, A., Kolev, G., \& Matthes, J. (2017). Wachstumsbremse Fachkräfteengpässe. Köln: Institut der deutschen Wirtschaft.

Campolieti, M., Gunderson, M. K., \& Smith, J. A. (2014). The effect of vocational rehabilitation on the employment outcomes of disability insurance beneficiaries: new evidence from Canada. IZA Journal of Labor Policy, 3(1), 1-29.

Cancelliere, C., Donovan, J., Stochkendahl, M. J., Biscardi, M., Ammendolia, C., Myburgh, C., \& Cassidy, J. D. (2016). Factors affecting return to work after injury or illness: Best evidence synthesis of systematic reviews. Chiropractic and Manual Therapies, 24(1), 32.

Card, D. (1999). The causal effect of education on earnings. In O. Ashenfelter \& D. Card (Eds.), Handbook of labor economics (Vol. 3, pp. 1801-1863). North Holland: Elsevier.

Card, D., Kluve, J., \& Weber, A. (2010). Active labour market policy evaluations: A meta-analysis. Economic Journal, 120(548), 452-477.

Cattaneo, M. D. (2010). Efficient semiparametric estimation of multi-valued treatment effects under ignorability. Journal of Econometrics, 155(2), 138-154.

Cattaneo, M. D., Arbor, A., Drukker, D. M., \& Holland, A. D. (2010). Estimation of multivalued treatment effects under conditional independence. The Stata Journal, (ii), 1-40. 
Cornelius, L. R., Van Der Klink, J. J. L. L., Groothoff, J. W., \& Brouwer, S. (2011). Prognostic factors of long term disability due to mental disorders: A systematic review. Journal of Occupational Rehabilitation, 21(2), 259-274.

Council of Europe. (1996). European Social Charter (Revised), 1996. Strasbourg.

Cullen, K. L., Irvin, E., Collie, A., Clay, F., Gensby, U., Jennings, P. A., ... Amick, B. C. (2018). Effectiveness of Workplace Interventions in Return-to-Work for Musculoskeletal, Pain-Related and Mental Health Conditions: An Update of the Evidence and Messages for Practitioners. Journal of Occupational Rehabilitation, 28(1), 1-15.

Dean, D. H., \& Dolan, R. C. (1991). Assessing the Role of Vocational Rehabilitation in Disability Policy. Journal of Policy Analysis and Management, 10(4), 568.

Dean, D., Pepper, J., Schmidt, R., \& Stern, S. (2015). The effects of vocational rehabilitation for people with cognitive impairments. International Economic Review, 56(2), 399-426.

Deeke, A., Dietrich, H., Kruppe, T., Lott, M., Rauch, A., Stephan, G., \& Wolff, J. (2011). Geförderte Qualifizierungsmaßnahmen in Deutschland: Aktuelle Evaluationsergebnisse im Überblick. Sozialer Fortschritt, 60(9), 196-203.

Dehejia, R. H., \& Wahba, S. (2002). Propensity score-matching methods for nonexperimental causal studies. Review of Economics and Statistics, 84(1), 151161.

Destatis. (2018). Consumer Price Index (including rates of change): Germany, Code: 611110001. Federal German Statistical Office.

Dodoo-Schittko, F., Brandstetter, S., Blecha, S., Thomann-Hackner, K., Brandl, M., Knüttel, H., ... Apfelbacher, C. (2017). Determinants of quality of life and return to work following acute respiratory distress syndrome - A systematic review. Deutsches Arzteblatt International, 114(7), 103-109.

Dolton, P., \& Smith, J. (2011). The Impact of the UK New Deal for Lone Parents The Impact of the UK New Deal for Lone Parents on Benefit Receipt. UK Department for Work and Pensions, (5491), 1-34.

Drummond, M. F., Sculpher, M. J., Claxton, K., Stoddart, G., \& Torrance, G. W. (2015). Methods for the Economic Evaluation of Health Care Programmes (4th ed.). Oxford: Oxford University Press.

DRV. (2017). Statistik der Deutschen Rentenversicherung: Rentenversicherung in 
Zahlen 2017. Statistik Der Deutschen Rentenversicherung. Berlin: Deutsche Rentenversicherung Bund.

Duggan, M., \& Imberman, S. A. (2008). Why Are the Disability Rolls Skyrocketing? The Contribution of Population Characteristics, Economic Conditions, and Program Generosity. In Health at Older Ages: The Causes and Consequences of Declining Disability among the Elderly (pp. 337-379). University of Chicago Press.

Dziak, J. J., Coffman, D. L., Lanza, S. T., \& Runze, L. (2012). Sensitivity and specificity of information criteria: Technical Report Series \#12-119. The Methodology Center, Technical Report, (814), 1-30.

Enichlmair, C., \& Heckl, E. (2013). Erfolg arbeitsmarktpolitischer Bildungsmaßnahmen: Angewandte Kriterien und Indikatoren am Beispiel Deutschland. Wien: KMU Forschung Austria.

Etuknwa, A., Daniels, K., \& Eib, C. (2019). Sustainable Return to Work: A Systematic Review Focusing on Personal and Social Factors. Journal of Occupational Rehabilitation.

Feuerstein, M., Berkowitz, S. M., Haufler, A. J., Lopez, M. S., \& Huang, G. D. (2001). Working with low back pain: Workplace and individual psychosocial determinants of limited duty and lost time. American Journal of Industrial Medicine, 40(6), 627-638.

Fitzenberger, B., \& Völter, R. (2007). Long-run effects of training programs for the unemployed in East Germany. Labour Economics, 14(4 SPEC. ISS.), 730-755.

Foreman, P., Murphy, G., \& Swerissen, H. (2006). Facilitators and Barriers to Return to Work: A Literature Review. South Australian WorkCover Corporation. Melbourne: Australian Institute for Primary Care, La Trobe University.

Franche, R.-L., Cullen, K., Clarke, J., Irvin, E., Sinclair, S., \& Frank, J. (2005). Workplace-Based Return-to-Work Interventions: A Systematic Review of the Quantitative Literature. Journal of Occupational Rehabilitation, 15(4), 607-631.

Frölich, M., Heshmati, A., \& Lechner, M. (2004). A microeconometric evaluation of rehabilitation of long-term sickness in Sweden. Journal of Applied Econometrics, 19(3), 375-396.

Furlan, A. D., Gnam, W. H., Carnide, N., Irvin, E., Amick, B. C., DeRango, K., ... Bültmann, U. (2012). Systematic review of intervention practices for depression in the workplace. Journal of Occupational Rehabilitation, 22(3), 312-321. 
Gericke, C. (2010). Einflussfaktoren der beruflichen Integration psychisch beeinträchtigter Menschen (Humboldt-Universität zu Berlin). Humboldt-Universität zu Berlin.

Giesen J., M., \& Cavenaugh Brenda, S. (2013). Disability Insurance Beneficiaries with Visual Impairments in Vocational Rehabilitation: Socio-demographic Influences on Employment. Journal of Visual Impairment $\mathcal{E}$ Blindness, 107(6), 453-467.

Glymour, M. M., Weuve, J., Berkman, L. F., Kawachi, I., \& Robins, J. M. (2005). When is baseline adjustment useful in analyses of change? An example with education and cognitive change. American Journal of Epidemiology, 162(3), 267278.

Greene, W. H. (2012). Econometric Analysis (6th ed.). New Jersey: Prentice-Hall.

Heckman, J. J., \& Robb, R. (1985). Alternative methods for evaluating the impact of interventions. An overview. Journal of Econometrics, 30(1-2), 239-267.

Hirano, K., \& Imbens, G. W. (2001). Estimation of causal effects using propensity score weighting: An application to data on right heart catheterization. Health Services and Outcomes Research Methodology, 2(3-4), 259-278.

Hirano, K., Imbens, G. W., \& Ridder, G. (2003). Efficient estimation of average treatment effects using the estimated propensity score. Econometrica, 71(4), 1161-1189.

Hoffmann, H. (2004). Berufliche Rehabilitation. In W. Rössler (Ed.), Psychiatrische Rehabilitation. Berlin, Heidelberg: Springer.

Hoosain, M., de Klerk, S., \& Burger, M. (2019). Workplace-Based Rehabilitation of Upper Limb Conditions: A Systematic Review. Journal of Occupational Rehabilitation, pp. 175-193.

Horvitz, D. G., \& Thompson, D. J. (1952). A Generalization of Sampling Without Replacement from a Finite Universe. Journal of the American Statistical Association, 47(260), 663-685.

Hosmer, D. W., Lemeshow, S., \& Sturdivant, R. X. (2013). Applied Logistic Regression: Third Edition (3rd ed.). Hoboken, NJ, USA: John Wiley \& Sons.

ILO. (1983). C159 Vocational Rehabilitation and Employment (Disabled Persons) Convention. Geneva: International Labour Organization.

Imbens, GW. (2004). Nonparametric estimation of average treatment effects under exogeneity: A review. Review of Economics and Statistics, 86(1), 4-29. 
Imbens, G. W. (2000). The role of the propensity score in estimating dose-response functions. Biometrika, 87(3), 706-710.

Imbens, G. W., \& Rubin, D. B. (2015). Causal Inference for Statistics, Social, and Biomedical Sciences: An Introduction. Cambridge: Cambridge University Press.

Imbens, G., \& Wooldridge, J. (2007). Estimation of Average Treatment Effects under unconfoundedness. Lecture Series: What's New in Econometrics. National Bureau of Economic Research.

ISSA. (2012). Social Policy Highlight 22. Geneva: International Social Security Association.

ISSA. (2014). Social Security Programs Throughout the World: Europe, 2014. Geneva: International Social Security Association.

Kardorff, E. Von, \& Ohlbrecht, H. (2013). Abbrüche beruflicher Qualifizierungsmaßnahmen in der Rehabilitation. 1-185.

Kreutzer, J. S., Marwitz, J. H., Walker, W., Sander, A., Sherer, M., Bogner, J., ... Bushnik, T. (2003). Moderating factors in return to work and job stability after traumatic brain injury. The Journal of Head Trauma Rehabilitation, 18(2), 128-138.

Laaksonen, M., \& Gould, R. (2015). Return to Work After Temporary Disability Pension in Finland. Journal of Occupational Rehabilitation, 25(3), 471-480.

Langi, F. L. F. G., Balcazar, F. E., \& Suarez-Balcazar, Y. (2018). Analysis of Time in Rehabilitation and Incidence of Successful Rehabilitation Within Individuals with Disabilities Receiving Occupational/Vocational Training. Journal of Occupational Rehabilitation, 28(4), 701-710.

Leinonen, T., Solovieva, S., Husgafvel-Pursiainen, K., Laaksonen, M., \& ViikariJuntura, E. (2019). b. PLOS ONE, 14(2), e0212498.

Linden, A., \& Yarnold, P. R. (2016). Combining machine learning and propensity score weighting to estimate causal effects in multivalued treatments. Journal of Evaluation in Clinical Practice, 22(6), 871-881.

Lindsay, S., Cagliostro, E., Albarico, M., Mortaji, N., \& Karon, L. (2018). A Systematic Review of the Benefits of Hiring People with Disabilities. Journal of Occupational Rehabilitation, Vol. 28, pp. 634-655.

Long, J. S., \& Freese, J. (2014). Regression Models for Categorical Dependent Variables Using STATA. Stata Press. 
Lysaght, R., Kranenburg, R., Armstrong, C., \& Krupa, T. (2016). Participant Recruitment for Studies on Disability and Work: Challenges and Solutions. Journal of Occupational Rehabilitation, 26(2), 125-140.

MacDonald, S. J., Brodner, W., \& Jacobs, J. J. (2004). A consensus paper on metal ions in metal-on-metal hip arthroplasties. The Journal of Arthroplasty, 19(8), 12-16.

Maris, E. (1998). Covariance adjustment versus gain scores-revisited. Psychological Methods, 3(3), 309-327.

McDowell, C., \& Fossey, E. (2015). Workplace Accommodations for People with Mental Illness: A Scoping Review. Journal of Occupational Rehabilitation, 25(1), 197-206.

Meschnig, A., von Kardorff, E., \& Klaus, S. (2019). Von der beruflichen Vollqualifizierungsmaßnahme zurück in ArbeitEine Langzeitanalyse individueller Verlaufskarrieren und ihrer biografischen und strukturellen Bedingungen. Die Rehabilitation, 58(03), 153-162.

Muijzer, A., Groothoff, J. W., Geertzen, J. H. B., \& Brouwer, S. (2011). Influence of efforts of employer and employee on return-to-work process and outcomes. Journal of Occupational Rehabilitation, 21(4), 513-519.

Muijzer, Anna, Groothoff, J. W., De Boer, W. E. L., Geertzen, J. H. B., \& Brouwer, S. (2010). The assessment of efforts to return to work in the European Union. European Journal of Public Health, 20(6), 689-694.

Neumann, M., \& Lenske, W. (2010). Endbericht Kosten und Nutzen der beruflichen Rehabilitation junger Menschen mit Behinderungen oder funktionalen Beeinträchtigungen - eine gesamtwirtschaftliche Analyse. Köln: Institut der deutschen Wirtschaft.

Nevala, N., Pehkonen, I., Koskela, I., Ruusuvuori, J., \& Anttila, H. (2015). Workplace Accommodation Among Persons with Disabilities: A Systematic Review of Its Effectiveness and Barriers or Facilitators. Journal of Occupational Rehabilitation, 25(2), 432-448.

Nikolic, I. A., Stanciole, A. E., \& Zaydman, M. (2011). Chronic Emergency: Why NCDs Matter. Health, Nutrition, and Population, (July), 34.

Odeen, M., Magnussen, L. H., Maeland, S., Larun, L., Eriksen, H. R., \& Tveito, T. H. (2013). Systematic review of active workplace interventions to reduce sickness absence. Occupational Medicine, 63(1), 7-16. 
OECD. (2009). Sickness, Disability and Work: Keeping On Track in the Economic Downturn. Stockholm: Organisation for Economic Co-operation and Development.

OECD. (2010). Sickness, disability and work: Breaking the barriers - A synthesis of findings across OECD countries. Paris: OECD Publishing.

Ottomanelli, L., \& Lind, L. (2009). Review of critical factors related to employment after spinal cord injury: Implications for research and vocational services. Journal of Spinal Cord Medicine, 32(5), 503-531.

Petrunyk, I., Pfeifer, C., Fischer, S., \& Wiemer, A. (2015). Before-After Differences in Labor Market Outcomes for Participants in Medical Rehabilitation in Germany. Schmollers Jahrbuch, 135(4), 537-562.

Robins, J. M., Rotnitzky, A., \& Zhao, L. P. (1995). Analysis of semiparametric regression models for repeated outcomes in the presence of missing data. Journal of the American Statistical Association, 90(429), 106-121.

Rosenbaum, Paul R, Rubin, D. B. (1984). Reducing Bias in Observational Studies Using Score on the Propensity Subolassification. The American Economic Review, 79(387), 516-524.

Rosenbaum, P. R., \& Rubin, D. B. (2006). The central role of the propensity score in observational studies for causal effects. Biometrika, 70, 170-184.

Rubin, D. B. (1974). Estimating causal effects of treatments in randomized and nonrandomized studies. Journal of Educational Psychology, 66(5), 688-701.

Saltychev, M., Tenovuo, O., \& Laimi, K. (2013). Return to work after traumatic brain injury: Systematic review. Brain Injury, 27(13-14), 1516-1527.

Schmidt, C., Flach, T., \& Begerow, B. (2011). Einfluss psychosozialer Faktoren auf die Wiedereingliederung in Arbeit. Köln: Institut für Qualitätssicherung in Prävention und Rehabilitation (iqpr) $\mathrm{GmbH}$ an der Deutschen Sporthochschule Köln.

Sears, J. M., Rolle, L. R., Schulman, B. A., \& Wickizer, T. M. (2014). Vocational Rehabilitation Program Evaluation: Comparison Group Challenges and the Role of Unmeasured Return-to-Work Expectations. Journal of Occupational Rehabilitation, 24(4), 777-789.

Selander, J., Marnetoft, S.-U., Bergroth, A., \& Ekholm, J. (2002). Return to work following vocational rehabilitation for neck, back and shoulder problems: risk 
factors reviewed. Disability and Rehabilitation, 24(14), 704-712.

SGB IX. Ninth book of the German Code of Social Law from 23 December 2016 (BGBl. I S. 3234 (Federal Law Gazette I, p. 3234)), which has most recently been amended through Article 5 of the law from 8 July (BGBl. I S. 1025 (Federal Law Gazette I, p. 1025)). , (2016).

Statistisches Bundesamt. (2017). Pressemitteilung Nr. 228. Wiesbaden.

Steenstra, I. A., Lee, H., De Vroome, E. M. M., Busse, J. W., \& Hogg-Johnson, S. J. (2012). Comparing current definitions of return to work: A measurement approach. Journal of Occupational Rehabilitation, 22(3), 394-400.

Stergiou-Kita, M., Mansfield, E., \& Sokoloff, S. (2016). Gender Influences on Return to Work After Mild Traumatic Brain Injury. Archives of Physical Medicine and Rehabilitation, 97(2), S40-S45.

Street, T. D., \& Lacey, S. J. (2015). A systematic review of studies identifying predictors of poor return to work outcomes following workplace injury. Work, 51(2), 373-381.

Streibelt, M., \& Egner, U. (2012a). Eine Meta-Analyse zum Einfluss von Stichprobe, Messmethode und Messzeitpunkt auf die berufliche Wiedereingliederung nach beruflichen Bildungsleistungen. Die Rehabilitation, 51(06), 398-404.

Streibelt, M., \& Egner, U. (2012b). Eine systematische Übersichtsarbeit zu den Einflussfaktoren auf die berufliche Wiedereingliederung nach beruflichen Bildungsleistungen. Die Rehabilitation, 52(02), 111-118.

Swiaczny, F. (2016). Demografischer Wandel und Migration in Deutschland. In Handbuch Diversity Kompetenz (pp. 155-172). Wiesbaden: Springer Fachmedien Wiesbaden.

UN General Assembly. (2007). Convention on the Rights of Persons with Disabilities : resolution / adopted by the General Assembly, 24 January 2007. A/RES/61/106.

Valentin, G. H., Pilegaard, M. S., Vaegter, H. B., Rosendal, M., Ørtenblad, L., Væggemose, U., \& Christensen, R. (2016). Prognostic factors for disability and sick leave in patients with subacute non-malignant pain: a systematic review of cohort studies. BMJ Open, 6(1), e007616.

van der Laan, M. J., \& Robins, J. M. (2003). Unified Methods for Censored Longitudinal Data and Causality. New York: Springer.

Van Muijen, P., Weevers, N. L. E. C., Snels, I. A. K., Duijts, S. F. A., Bruinvels, D. J., 
Schellart, A. J. M., \& van der Beek, A. J. (2013). Predictors of return to work and employment in cancer survivors: A systematic review. European Journal of Cancer Care, 22(2), 144-160.

van Vilsteren, M., van Oostrom, S. H., de Vet, H. C. W., Franche, R. L., Boot, C. R. L., \& Anema, J. R. (2015). Workplace interventions to prevent work disability in workers on sick leave. Cochrane Database of Systematic Reviews, 2015(10), CD006955.

Weber, A., Peschkes, L., \& de Boer, W. (2015). Return to Work (RtW) Begriffsbestimmung und Hintergrund. In Return to Work - Arbeit für alle. Grundlagen der beruflichen Reintegration (pp. 23-34). Stuttgart: Gentner Verlag.

White, M. I., Wagner, S. L., Schultz, I. Z., Murray, E., Bradley, S. M., Hsu, V., ... Schulz, W. (2015). Non-modifiable worker and workplace risk factors contributing to workplace absence: A stakeholder-centred synthesis of systematic reviews. Work, 52(2), 353-373.

Wooldridge, Jeffrey, M. (2010). Econometric Analysis of Cross Section and Panel Data (2nd ed.). Cambridge, MA: MIT Press.

Wooldridge, J. M. (2007). Inverse probability weighted estimation for general missing data problems. Journal of Econometrics, 141(2), 1281-1301.

World Health Organization \& World Bank. (2011). The World Report on Disability 2011. World Health Organization.

Yasuda, S., Wehman, P., Targett, P., Cifu, D. X., \& West, M. (2002). Return to work after spinal cord injury: a review of recent research. NeuroRehabilitation, 17(3), 177-186. 


\section{Summary}

This thesis adds to the growing body of literature on the effects of labor market activation programs for people with a disability. Work disability is nowadays widely viewed as a public health issue, where the consequences of work disability are considered not only to be following a biomedical causality but also to be influenced by participation restrictions and contextual factors. It is widely accepted that many of the barriers facing people with disabilities are, in fact, avoidable and that workers with a disability are far from incompatible with employment in the labor market. Factors such as the absence of access to vocational education and retraining and the lack of financial and other assistance have been found to be significant drivers of labor market exclusion. Consequently, to improve chances for re-employment, despite physical limitations and other health restrictions, vocational rehabilitation and re-training programs have been implemented by social policymakers in many parts of the world. However, so far, much of the empirical evidence on the effects of vocational rehabilitation programs has been confined to small sample sizes and specific institutional settings. In order to improve the empirical understanding of the work participation effects of these programs this dissertation project has been initiated.

In this thesis, unique administrative data, a relevant time of follow-up and advanced statistical measures to handle confounding have been utilized in order to provide new and informative evidence on the effects of vocational rehabilitation programs on work participation outcomes in Germany. While re-affirming the important role of micro-level determinants, the present study provides an extensive example of the individual and fiscal effects that are possible through meaningful vocational rehabilitation measures. The impact analysis presented in this thesis takes a broad perspective and examines the influence of vocational re-training on the number of employment days, income earned, days on unemployment and other social-security benefits and on the likelihood of receiving an earnings incapacity pension. The research questions of this thesis were as follows:

1. To what extent do individual socio-economic factors influence return-towork outcomes after participation in vocational rehabilitation?

2. To what extent do vocational re-training programs impact employment outcomes and influence dependency on social security benefits?

3. Are there significant differences between the effects of one- and two-year retraining programs with regards to income earned, employment days and social security benefit receipt?

4. What are the financial returns for providers of the re-training programs: are the measures cost-effective? 
The thesis comprises six chapters. In the first chapter the aims and contributions of the thesis as well as the study context and research methods were introduced.

In the second chapter, employment outcomes and their determinants after participation in vocational rehabilitation have been investigated through an analysis of a representative panel dataset of German rehabilitants. Since participants in vocational rehabilitation are a heterogeneous group of people, with different social and occupational backgrounds and different health situations, possible barriers to work reintegration can only adequately be assessed with reference to the rehabilitants' individual situations. A needed step to improve the empirical evidence is, thus, to assess the relevant person-related factors influencing return-towork outcomes. This chapter provides an overview of the direction and size of the relevant person- and occupation-related factors on work participation outcomes. The analysis included identifying which factors positively affected re-employment chances and which socio-economic characteristics tended to act as barriers to successful work reintegration.

The evaluated factors comprised age, gender, education, employment history, job type as well as other relevant socio-economic characteristics. The analysis of socioeconomic determinants also served as a starting point for subsequent impact analysis since to know the effect of socio-economic factors on employment outcomes is a necessary condition for validly examining an interventions' impact. Among the different factors tested, personal employment and income history were the strongest predictors. Additionally, other factors, such as the rehabilitants' age, prior education level or work environment were found to have a strong impact. The attained findings in this chapter support the argument that providers of vocational rehabilitation services need to be aware of the influence demographic- and occupational factors have on re-employment chances: the socio-economic status and the work environment significantly affect return-to-work outcomes after participation in vocational rehabilitation.

In the third chapter, an empirical example of how to use administrative panel data when evaluating re-training programs for people with disabilities has been provided. It was examined, to what extent completing a vocational re-training, the most time-intensive and costly type of vocational rehabilitation measure, impacted employment outcomes and to what extent the programs reduced the likelihood of receiving an earnings incapacity pension. In the absence of randomization, an applicant-based comparison group was used to compare outcomes with and without completion of vocational re-training. While the selection of potential controls was one of the key challenges to estimate the treatment effects, advanced 
statistical measures to handle confounding were applied in order to establish comparability.

The first result of the impact analyses carried out was that completing vocational retraining greatly improved the employment status of the rehabilitants, i.e., the programs led to significantly more days in employment and significantly higher accumulative income, in the first eight years after program start. Secondly, completing a re-training program also reduced the number of days on unemployment and other social security benefits and the likelihood of receiving a pension due to reduced earnings capacity. The estimated treatment effects provide policymakers with detailed information about the interventions' abilities to improve the individuals' employment statuses and show to what extent these improvements were sustained over-time. In addition to its empirical relevance, the research also contributes to the methodological debate on how to estimate the impact of labor market programs on employment outcomes from observational data using quasiexperiments. This approach, so far, has been widely underutilized in vocational rehabilitation research in Germany up to this point.

In the fourth chapter, it was examined, to what extent one-year and two-year vocational re-training programs had a similar impact on employment outcomes when directly compared with each other. In the light of political initiatives to strengthen horizontal training, one-year re-training measures are becoming increasingly popular as an alternative to the more involved two-year re-training programs. Comparing both program types directly, this chapters' analysis showed that the long-term employment effects associated with the traditional, two-year retraining programs were significantly larger compared to one-year re-training programs. However, accumulative differences in income and employment days eight years after program start, were not statistically significant. The duration of transfer payments was characterized by shifts, i.e. being allocated a two-year retraining was linked to fewer days with long-term unemployment benefits but more days with short-term unemployment benefits and more days with other social security benefits. Whereas the increase in days with other social security-benefits was mainly due to the longer program duration, the shift in benefit recipience may have been advantageous, as long-term unemployment was reduced substantially.

In chapter five, the vocational re-training costs and benefits to social security providers were compared to evaluate the cost-effectiveness and efficiency of vocational re-training. By providing new evidence on metrics such as the net present value, the return on investment and the internal rate of return, this chapter's analysis contributes to a better understanding of the economic consequences of vocational 
re-training. Taking a long-term perspective, it has been shown after how many years the re-training programs break even and how large the economic returns to the social security system were. The estimated financial balance sheets showed that both one-year and two-year vocational re-training programs were, on average, costeffective; however, only after a substantial payback period. Another result from this analysis was that the financial payback period of partial re-training was shorter in comparison to the longer full-retraining measures.

The sixth chapter presents the conclusions of this thesis. It comprises a summary and discussion of the gathered evidence with regards to answering the four research questions posed in the introduction as well as a discussion on policy implications, possible limitations of this study and recommendations for future research. Taken together, the German vocational re-training program for people with a disability has been found to have a positive impact on rehabilitants' employment outcomes. The results showed that the principal objective, namely, to improve participation in employment, was generally achieved. Contrary to the common misconception that "off-the-job training" is relatively ineffective, this thesis has provided an empirical example of the positive impact of the programs.

The following section summarizes the main findings in order to answer the four research questions posed in the introduction section of this thesis:

1. To what extent do individual socio-economic factors influence return-to-work outcomes after participation in vocational rehabilitation?

According to the analysis in chapter two, the socio-economic status plays a substantial role in an individual's return to work. The measured differences in employment outcomes, after participation in vocational rehabilitation, were closely related to the participants' socio-demographic and employment characteristics. The attained findings in this analysis support the argument that providers of vocational rehabilitation services need to be aware of the influence individual-level socioeconomic factors have on employment outcomes in the return to work of people with a disability.

2. To what extent do vocational re-training programs impact employment outcomes and influence dependency on social security benefits?

The results of this analysis suggest that, over the first eight years after program admission, both types of vocational re-training programs analyzed significantly improved the income and employment situation of the individuals involved. According to the estimated treatment effects in chapter three, completing a 
vocational re-training was associated with significant improvements in the employment status while also reducing dependency on social security benefits. Policies to promote the labor market re-integration should consider that vocational re-training may be an effective tool for sustainably improving the employment situation of people with a disability.

3. Are there significant differences between the effects of one- and two-year retraining programs with regards to income earned, employment days and social security benefit receipt?

While annual long-term results were highly statistically significant, the accumulative results over the first eight-years after program admission were not statistically significant due to negative lock-in effects at the beginning of the observation period, linked to longer program duration. Nevertheless, the analysis showed that the disadvantages of longer program duration were generally offset by higher employment outcomes in the long term for participants of two-year retraining programs. From the perspective of the re-training participants, the additional year of re-training generally paid off, i.e. the treatment effects on the treated with regards to income and employment days were positive for two-year retraining participants and the likelihood to have been awarded a pension due to a reduced earnings capacity was also lower. In addition, a significant and large reduction in days with long-term unemployment benefits indicates that two-year retraining programs had a significantly greater impact with regards to preventing permanent exit from the labor market.

4. What are the financial returns for providers of the re-training programs: under what conditions are the measures cost-effective?

Both types of re-training measures evaluated were, on average, cost-effective from the social security perspective. Despite the restrictive assumptions used, the estimated balance sheets demonstrate the large economic potential of investing in work reintegration measures. From the social security perspective, the average net present value of providing one- and two-year re-training services were positive in the long run, however, only after a substantial payback period. While the payback period of one-year programs was assessed to be much shorter, a possible explanation for the current expansion of horizontal training is given. The policymakers and the individual's interest may, thus, conflict in some circumstances. This is because the individual might prefer the, more involved, twoyear measure, but is only allocated, a less costly and more cost-efficient one-year measure. 
The policy recommendations derived from this study are summarized as follows:

1. Provide individualized assistance. This study has shown that factors such as greater age or low prior educational attainments decrease the chances of a successful return to employment. To improve return-to-work outcomes, it is therefore recommended that policy targets these groups. For example, by improving access to and the effectiveness of individual case management.

2. Continue to invest in vocational re-training as it generates a positive impact on return-to-work outcomes.

3. Prevent program dropout as this leads to significantly worse work participation outcomes and, thereby, improve the overall effectiveness and efficiency of retraining measures. Since about half of the dropouts were related to medical reasons, better a priori screening and better medical supervision during the programs may contribute to reducing program dropout rates. Regarding the non-medical dropouts, many were due to performance issues and failed examinations. Further investigation is needed into why programs that were deemed to be appropriate turned out to be infeasible for certain individuals.

4. Re-assess program allocation criteria. The literature on the comparative effects of one- and two-year re-training has previously found that reduced training may lead to better outcomes. A shortening of the average duration of measures tends to have a favorable effect in the short term because the lock-in effect loses weight compared to the program effect. However, when assessed with longer followup, the more involved training programs have been found to be more effective since the long-term outcomes were significantly better. These results ask for reassessing the conditions under which comparable candidates who are being given a partial re-training could be upgraded to a full re-training measure. This change could potentially improve individual employment outcomes and, thus, also benefit the social security system in the long run.

5. Use the provided empirical evidence to strengthen the role of vocational rehabilitation in the public debate. Vocational re-training was generally costeffective when compared to the alternative of no completion of re-training since it increases wages and employment days while reducing the number of days on social security and unemployment benefits. Accordingly, policymakers should make use of the provided empirical evidence to strengthen the role of vocational re-training with regards to its usage and application within the overall social protection system - vocational rehabilitation pays off, also from the social security perspective. 


\section{Valorization addendum}

In this addendum, the relevance of this thesis for policy and practice is described.

Work disability is a challenge for social security systems, affecting millions of people worldwide. At the same time, social policymakers have implemented vocational rehabilitation programs aimed at improving the chances for re-employment despite physical limitations and other health restrictions. So far, much of the empirical evidence on the effects of vocational rehabilitation in Germany has been confined to small sample sizes and specific institutional settings. In this thesis, unique administrative data, a relevant time of follow-up and advanced statistical measures to handle confounding effects have been utilized in order to provide new and informative evidence on the effects of vocational rehabilitation on employment outcomes. This evidence is needed for policymakers to be able to strengthen the role and effectiveness of vocational rehabilitation programs.

This thesis has made four major contributions to the analysis of the effectiveness of vocational rehabilitation programs for persons with disabilities:

In chapter two it was investigated how socio-economic factors influenced the employment outcomes after participation in vocational rehabilitation. The analysis included identifying which factors positively affect re-employment chances and which socio-economic characteristics tend to act as barriers to successful work reintegration. Several risk factors were consequently identified that were associated with little improvement in the employment status: Among the different factors tested, old-age, low prior educational outcomes and irregular employment relationships were especially associated with less improvement in the employment status. The attained findings in this chapter support the argument that providers of vocational rehabilitation services need to be aware of the influence demographicand occupational factors have on re-employment chances: the socio-economic status and the work environment significantly affect return-to-work outcomes after participation in vocational rehabilitation. This study has shown that factors such as greater age or low prior educational attainments decrease the chances of a successful return to employment. To improve work participation outcomes, it is therefore recommended that policy targets these groups. For example, by improving access to and the effectiveness of individual case management; for case managers it is particularly important to consider the effects of the identified contextual factors as they shape the values and goals of the individuals and influence the outcome of the work reintegration process. 
In chapter three the impact of re-training was assessed through a comparison with similar program dropouts. While taking the effect of confounding variables into account, the analyses examined to what extent the vocational re-training program, the most time-intensive and costly type of vocational program available, impacted employment outcomes and to what extent the programs reduced the likelihood of receiving an earnings incapacity pension. The conclusion of the analysis was that the vocational re-training programs analyzed had a positive impact on rehabilitants' work participation outcomes. The estimated treatment effects provide policymakers with detailed information about the interventions' abilities to improve the individuals' employment statuses and show to what extent these improvements were sustained over-time: Despite the presence of physical limitations, completing the re-training measures greatly improved the employment situation of the rehabilitants, i.e. graduating from a re-training program resulted in more days in employment and higher annual income earned while at the same time dependency on social security benefits was reduced.

The analysis showed that the principal objective, namely, to improve participation in employment, was generally achieved. Contrary to the common misconception that "off-the-job training" is relatively ineffective, this thesis has provided an empirical example of the positive impact of the programs. Therefore, a policy recommendation offered by this thesis is to continue to invest in vocational retraining as it generates a positive impact on participating individuals. The gathered evidence in this thesis is not only of interest to those who are currently undergoing re-training or those that are planning to do so in the future, but also gives the implementing agencies a detailed evaluation of the impact of their work. In addition to its empirical relevance, the research also contributes to the methodological debate on how to estimate the impact of labor market programs on employment outcomes from observational data using quasi-experiments. This approach, so far, has been widely underutilized in vocational rehabilitation research in Germany and can hopefully inspire other researchers to embark on similar research projects.

Another relevant conclusion from the analysis in chapter three is that program dropout was associated with significantly worse employment outcomes, compared to program graduation. Unsuccessful completing seems to be a strong predictor for worse work participation outcomes. Accordingly, preventing dropout and, thus, improving the overall effectiveness and efficiency of re-training measures should be an important policy target. Since about half of the dropouts were related to medical reasons, better a priori screening and medical supervision during the programs may contribute to reducing program dropout rates. Regarding the non-medical dropouts, many were due to performance issues and failed examinations. As such it would be 
worthwhile to further explore why the individuals did not finish the programs and what type of assistance could be provided to increase graduation rates. Moreover, the outcome of allocation practices should be further examined: Are the individuals satisfied with the occupational re-training choice? Would they rather have enrolled in a different trade or occupation? Are there other reasons that influence how well the rehabilitants perform in the examinations? Given the large benefits associated with completed re-training and the comparatively large costs of providing the measures, these are relevant questions.

In chapter four, it was examined, to what extent one-year and two-year vocational education programs have similar effects on employment outcomes, when directly compared with each other. The literature on the comparative effects of one- and twoyear re-training has previously found that reduced training may lead to better outcomes: a shortening of the average duration of measures tends to have a favorable effect in the short term because the lock-in effect loses weight compared to the program effect. However, when assessed over a longer time period, the more involved training programs have been found to have a larger impact on work participation outcomes. The analysis in this thesis showed that the disadvantages of longer program duration were offset by higher employment outcomes in the longterm for participants of two-year re-training programs. From the perspective of twoyear re-training participants, the additional year of re-training, thus, generally paid off, i.e. the treatment effects on the treated with regards to income and employment days were positive for two-year re-training participants and the likelihood to be awarded a pension due to a reduced earnings capacity was also lower. These results are an important reassurance, that the additional time invested into the longer training measure actually paid off for the individuals involved.

Furthermore, the retrieved results indicate that program allocation was, also, to some extent effective. The average treatment effect on the treated of two-year program participants was larger compared to the sample average treatment effect across both treatment groups and the average treatment effect on the treated of the one-year program participants (which shows the income loss associated with a reduced duration of training). Thus, the average participant that was allocated into two-year re-training benefitted more from the measures, than the average participant that was allocated a one-year re-training would have. While these estimates are based on averages and are a product of econometric analysis, further explorative analysis is needed to examine the conditions under which participants of one-year programs could profit from an increased duration of re-training. 
In chapter five, the cost-effectiveness and efficiency of re-training were evaluated from social security provider's perspective. The three prior chapters showed what impact vocational rehabilitation may have on the individuals involved; in the fifth chapter, secondary effects for social security were calculated. These comprised increased contributions and tax collection and a reduction in the paid compensation benefits. The cost-benefit analysis carried out provides new evidence on the secondary effects of vocational re-training (on social security). While oftentimes the costs of rehabilitation and work reintegration measures dominate public discussions, the cost-benefit analysis in this study puts the costs and benefits into comparison. The result of this analysis was that vocational re-training was costeffective when compared to the alternative of no completion of re-training. However, in relation to earlier studies, that usually only considered few years of follow up, this study has shown that a much longer post-treatment observation period is needed for the benefits of the programs to outweigh the costs. 


\section{About the Author}

Nicolas Echarti was born on February 18 ${ }^{\text {th }}, 1989$ in Mainz, Germany. He grew up in a small town near Darmstadt, Germany, where he attended high school from 2000 to 2008. After an exchange year in the USA, he obtained the German Abitur in 2008. Before entering university, Nicolas took a gap year to do voluntary work in the social sector. He then moved to Maastricht and obtained a bachelor's degree in International Economic Studies at the School of Business and Economics of Maastricht University, the Netherlands (2012), and a master's degree in Public Policy and Human Development at Maastricht Graduate School of Governance (2013). During his bachelor's degree, he spent one semester abroad at the University of Western Australia in Perth, Australia. After his master's degree, Nicolas moved to Bonn, Germany to do an internship at the social protection department of the German Development Cooperation (GIZ). In 2014, he started working for the German Statutory Accident Insurance (DGUV) on a research project about the economic returns of work reintegration measures. In the same year, he also started his PhD program in Governance and Policy Analysis at the Maastricht Graduate School of Governance, combining his work for the DGUV with obtaining a PhD degree. During his three-year project work for DGUV, Nicolas was invited to speak at several conferences, including the World Congress on Safety and Health at Work in 2017. In 2018, he joined the University of Applied Sciences Bonn-Rhein-Sieg (HBRS) for a curriculum evaluation and development project and to teach an undergraduate course in applied econometrics. 


\section{UNU-MERIT/MGSoG Dissertation Series}

2019

\section{Shellie E. Solomon}

Neighborhoods Matter:

Crime, collective efficacy and

foreclosures in Miami

UNU-MERIT/MGSoG Dissertation

Series № 235

\section{Michał Kazimierczak}

Regional Innovation and

Entrepreneurship

Patents, trade marks, entry and

entrants' growth in European

manufacturing industries

UNU-MERIT/MGSoG Dissertation

Series № 234

\section{Fernanda Soares}

The Influence of Within School and Across Schools' Collaborative Practices on Student Learning and Teaching

Outcomes in West Africa

UNU-MERIT/MGSoG Dissertation Series № 233

\section{Mira Bierbaum}

New Mindsets to Innovate Activation UNU-MERIT/MGSoG Dissertation Series № 232

\section{Norman Dytianquin}

Technology in the Asian Miracle and Crisis Debates: Applications of and Insights from the Field of Influence Approach to Input-Output Analysis UNU-MERIT/MGSoG Dissertation Series № 231

\section{Nga Le}

The implications of health insurance for the labour market and patient satisfaction with medical care in Vietnam

UNU-MERIT/MGSoG Dissertation Series № 230

\section{Jinhyuck Park}

Intellectual Property right protection and cross-border RED investments by multinational enterprises UNU-MERIT/MGSoG Dissertation Series № 228

\section{Richard de Groot}

Show me the Money:

Essays on the Impact of Cash Transfers on Child Nutrition and the Role of Intra-Household Dynamics UNU-MERIT/MGSoG Dissertation Series № 228 


\section{Catie Lott}

Diamonds are a Women's Best Friend

Broadening Measures of Women's

Access to Formal Political Decision-

Making

UNU-MERIT/MGSoG Dissertation

Series № 227

\section{Ana Cristina Calderon Ramirez}

Public Management Reforms

Three stories about public procurement agencification in Latin America UNU-MERIT/MGSoG Dissertation Series № 226

\section{Camilo Nicanor Carrillo Purin}

Teachers' in-service training and student achievement:

The effect of in-service training of

Peruvian teachers on student

achievement

UNU-MERIT/MGSoG Dissertation

Series № 225

\section{Hugo Confraria}

Developing scientific capacity in the

Global South

UNU-MERIT/MGSoG Dissertation

Series № 224

\section{Alison Cathles}

Educational Pathways and Skills:

Past, Present, and Future

UNU-MERIT/MGSoG Dissertation

Series № 223

\section{Ibrahima Sory Kaba}

Aggregate Fluctuations and

Development: Essays on

Macroeconomic Volatility and

Economic Growth

UNU-MERIT/MGSoG Dissertation

Series № 222

\section{Charlotte Keijser}

Firm Participation, Learning and

Innovation in Heterogenous Value

Chains of IT-enabled Services

UNU-MERIT/MGSoG Dissertation

Series № 221

\section{Salih Çevikarslan}

Innovation Strategies and Their

Implications for Technological Change

and Market Outcomes:

An Evolutionary Multi-Agent Based

Modelling Approach

UNU-MERIT/MGSoG Dissertation

Series № 220

\section{Wondimagegn Mesfin Tesfaye}

Essays on the Impacts of Climate-

Smart Agricultural Innovations on

Household Welfare

UNU-MERIT/MGSoG Dissertation

Series № 219

\section{Tatevik Poghosyan}

How Board Networks Affect Firm

Performance and Innovation Incentives in Transition Economies: The Case of Armenia

UNU-MERIT/MGSoG Dissertation

Series № 218 


\section{Arip Muttaqien}

Essays on Inequality and Polarization:

Empirical Studies in Developing Asia

UNU-MERIT/MGSoG Dissertation

Series № 217

2018

\section{Katrin Marchand}

Essays on Forced Migration and

Labour Market Participation in

Developing Countries

UNU-MERIT/MGSoG Dissertation

Series № 216

\section{Ortrun Merkle}

The Myth of Gender Neutral Power:

Corruption and Gender Norms

UNU-MERIT/MGSoG Dissertation

Series № 215

\section{Biljana Meshkovska}

Life after Trafficking:

(re)integration processes of women that have been trafficked for the purpose of sexual exploitation in Europe

UNU-MERIT/MGSoG Dissertation

Series № 214

\section{Vincenzo Vinci}

The Relevance of Institutions and

People's Preferences for Social

Protection

UNU-MERIT/MGSoG Dissertation

Series № 213

\section{Silke Heuser}

The Effectiveness of Environmental Policies on Reducing Deforestation in the Brazilian Amazon

UNU-MERIT/MGSoG Dissertation

Series № 212

\section{Jennifer Waidler}

Social Assistance and Remittances and Their Role in the Fight Against

Poverty

UNU-MERIT/MGSoG Dissertation

Series № 211

\section{Choolwe Muzyamba}

The role of community mobilization in the promotion of maternal health of women living with HIV in Zambia UNU-MERIT/MGSoG Dissertation Series № 210

Juan Carlos A. Castillo Sánchez Assessing the Role of the Export Sector in Mexican Economic

Development,1965-2014

UNU-MERIT/MGSoG Dissertation

Series № 209

\section{Tareq Abuelhaj}

Food Security Policy Impact Analysis:

The Econometrics of Cash and Food

Assistance Cost Effectiveness

UNU-MERIT/MGSoG Dissertation

Series № 208

\section{Marta Férnandez de Arroyabe}

Arranz

Essays on MEAS and Innovation

UNU-MERIT/MGSoG Dissertation

Series № 207 


\section{Clotilde Mahé}

Essays on Migration and Occupational

Choice

UNU-MERIT/MGSoG Dissertation

Series № 206

\section{Simone Sasso}

Talent on the move. Essays on Human Capital, Graduate Mobility and

Economic Development

UNU-MERIT/MGSoG Dissertation

Series № 205

\section{Khaled Walid Rajab}

Strategic Planning under Fragility

UNU-MERIT/MGSoG Dissertation

Series № 204

\section{Mutinta Hambayi Nseluke}

A Tall Order: Improving Child Linear Growth

UNU-MERIT/MGSoG Dissertation Series № 203

Elvis Korku Avenyo

Innovations and Firm Performance

in sub-Saharan Africa: Empirical

Analyses

UNU-MERIT/MGSoG Dissertation

Series № 202

\section{Ni Zhen}

Employment Dynamics, Firm

Performance and Innovation

Persistence in the Context of

Differentiated Innovation Types:

Evidence from Luxembourg

UNU-MERIT/MGSoG Dissertation

Series № 201

\section{Caroline Wehner}

Too Scared to Achieve: The Relation

Between Neuroticism,

Conscientiousness

and Socioeconomic Outcomes

UNU-MERIT/MGSoG Dissertation

Series № 200

\section{Stefania Innocenti}

On Institutional Persistence

UNU-MERIT/MGSoG Dissertation

Series № 199

Hassen Abda Wako

Economic Globalization, Institutions

and Development: Essays on Aid,

Foreign Direct Investment and Trade

UNU-MERIT/MGSoG Dissertation

Series № 198

2017

\section{Hans-Erik Edsand}

Winds of Change

UNU-MERIT/MGSoG Dissertation

Series № 197

\section{Ana Patricia Silva Vara}

Redressing the Gender Gap

UNU-MERIT/MGSoG Dissertation

Series № 196

\author{
Andrés Iván Mideros Mora \\ Essays on the Economic Effects of Non- \\ contributory Social Protection \\ UNU-MERIT/MGSoG Dissertation \\ Series № 195
}




\section{Tobias Broich}

New Actors in the Global Economy

UNU-MERIT/MGSoG Dissertation

Series № 194

\section{Bernard Nikaj}

From No-government to E-government UNU-MERIT/MGSoG Dissertation Series № 193

\section{Ali Safarnejad}

Prioritizing the HIV Response

UNU-MERIT/MGSoG Dissertation

Series № 192

\section{Clovis Freire}

Diversification and Structural

Economic Dynamics

UNU-MERIT/MGSoG Dissertation

Series № 191

\section{Michael Verba}

Innovation and Knowledge Dynamics:

Essays on the Knowledge Economy

UNU-MERIT/MGSoG Dissertation

Series № 190

\section{Pui Hang Wong}

The Hearts and Minds in Conflict and Peace: The Economics of

Counterinsurgency and the Psychology of Reconstruction

UNU-MERIT/MGSoG Dissertation Series № 189

\section{Brenda Yamba}

Schooling Despite All Odds: Evidence from Lesotho on Female Child Carers who Stayed in School UNU-MERIT/MGSoG Dissertation Series № 188

\section{Sheng Zhong}

Moving towards An Energy Efficient

Future: Essays on Energy Efficiency,

Technology and Development

UNU-MERIT/MGSoG Dissertation

Series № 187

\section{Julieta Marotta}

Access to Justice and Legal

Empowerment of Victims of Domestic

Violence through Legal Organizations

in the City of Buenos Aires: A

Qualitative Empirical Legal Study

UNU-MERIT/MGSoG Dissertation

Series, № 186

\section{Andrea Franco-Correa}

On the Measurement of

Multidimensional Poverty as a Policy

Tool: Empirical Applications to Chile, Colombia, Ecuador and Peru

UNU-MERIT/MGSoG Dissertation

Series, № 185

\section{6}

\section{Yesuf Awel}

Insurance for Growth: Empirical Essays on Insurance Demand and Impacts in Africa

UNU-MERIT Dissertation Series, № 108

\section{Tigist Mekonnen Melesse}

Grow More Food using Fewer

Resources: Agricultural Technology

Adoption and Innovation Practices for

Inclusive and Sustainable

Development

UNU-MERIT Dissertation Series, № 107 


\section{Eleni Yitbarek}

Getting Ahead or left Behind? Essays on Poverty Dynamics and Social

Mobility in Africa

UNU-MERIT Dissertation Series, № 106

\section{Thuy Dieu Nguyen}

Firm-Level Theory and Evidence of

Corruption

UNU-MERIT Dissertation Series, № 105

\section{Raquel Tsukada Lehman}

Essays on Household Production with

Labor-Saving Technology

UNU-MERIT Dissertation Series, № 104

\section{Eva Barteková}

Multi-Problem Challenges for a

Renewable Future: Empirical Studies on Competitive Disadvantages from

Electricity Price Differentials and

Mineral Supply Risk in an Open

Economy

UNU-MERIT Dissertation Series, № 103

\section{Jocelyn Olivari}

Entrepreneurial Traits and Innovation:

Evidence from Chile

UNU-MERIT Dissertation Series, № 102

\section{Muhammad Shafique}

Essays on the role of knowledge, RED, and Technology-based Firms in the Evolution of Socio-techno-economic System

UNU-MERIT Dissertation Series, № 101

\section{Serdar Türkeli}

Governance of Innovation Policy:

Empirical Studies on Applied Political Economy by Multi-Methods Analysis UNU-MERIT Dissertation Series, № 100

\section{Ayokunu Adedokun}

Pathways to Sustainable Peace building in Divided Societies: Lessons and Experiences from Mozambique MGSoG Dissertation Series, № 75

\section{Luiz Rothier Bautzer}

Organizing Concurrent Engineering through ICT Platforms

Blueprinting Product Lifecycle

Management Platforms across

Disciplinary Agencies

MGSoG Dissertation Series, № 74

\section{Natalia Popova}

Migration in the Periphery of the

European Union:

Determinants of Successful and

Sustainable Labour Market Integration of Return Migrants in Albania, Egypt, Moldova and Tunisia

MGSoG Dissertations Series, № 73 


\section{Richard A. Martina}

Uncertainty and Resource Constraint in the Small Island Developing States: Essays in Entrepreneurial Cognition MGSoG Dissertations Series, № 72

\section{Cécile Cherrier}

The Expansion of Basic Social

Protection in Low-income Countries: An Analysis of Foreign Aid Actors' Role in the Emergence of Social Transfers in Sub-Saharan Africa MGSoG Dissertations series, № 71

\section{Paul Caldron}

The Tacit Bargain in Short-Term Medical Missions: Why U.S. physicians go and what it costs MGSoG Dissertation Series, № 70

\section{Mahmut Kobal}

Customs \& Excellence: A Comparative Approach on Administrative and Regulatory Compliance Perspectives of the EU-Turkey Customs Union MGSoG Dissertation Series, № 69

\section{Craig Loschmann}

Essays on Conflict-related Migration and Development in the Case of Afghanistan MGSoG Dissertations Series, № 68

\section{Andrea Milan}

Rural Livelihoods, Location and Vulnerable Environments: Approaches to Migration in Mountain areas of Latin America MGSoG Dissertation Series, № 67

\section{Farida Lada}

On Guarding the Welfare of Clinical Trial Subjects While Promoting Novel Drug Innovation

A Game Theoretical Approach MGSoG Dissertation Series, № 66

\section{5}

\section{Hibret Belete Maemir}

Dissecting Aggregate Productivity: International Integration and Growth with Heterogeneous Firms

UNU-MERIT Dissertation Series, № 96

\section{Giorgio Triulzi}

Looking for the Right Path: Technology Dynamics, Inventive Strategies and Catching-up in the Semiconductor Industry

UNU-MERIT Dissertation Series, № 95
Abdul Baseer Qazi
Knowledge flows and networks in the ICT sector: The case of Pakistan
UNU-MERIT Dissertation Series, № 94
Ajay Thutupalli
Technology Paradigm Shifts in Agriculture: Drivers of Sustainability and Catch up UNU-MERIT Dissertation Series, № 93 


\section{Eduardo Urias}

Improving access to HIVIAIDS

treatment in Brazil: When are

Compulsory Licenses effective in Price

Negotiations?

UNU-MERIT Dissertation Series, № 92

\section{Francesca Guadagno}

Why have so few Countries

Industrialised?

UNU-MERIT Dissertation Series, № 91

\section{Daniel Opolot}

The Evolution of Beliefs and Strategic

Behaviour

UNU-MERIT Dissertation Series, № 90

\section{Alejandro Lavopa}

Structural Transformation and

Economic Development: Can

Development Traps be Avoided

UNU-MERIT Dissertation Series, № 89

\section{Jinjin Zhao}

Urban water management reform: The Case of China

UNU-MERIT Dissertation Series, № 88

\section{Simona Vezzoli}

Borders, Independence and Postcolonial Ties: the Role of the State in Caribbean Migration MGSoG Dissertation Series, № 65

\section{Silvia Consuelo Gómez Soler}

Civil Conflict and Education: How

Does Exposure to Civil Conflict Affect

Human Capital Accumulation?

Evidence from Standardized Exit

Exams in Colombia

MGSoG Dissertation Series, № 64

\section{Paula Nagler}

Occupational Choice in the Developing World

MGSoG Dissertation Series, № 63

\section{Jasmin Kientzel}

Determinants of Professional

Commitment to Environmental

Sustainability

MGSoG Dissertation Series, № 62

\section{Mehmet Güney Celbiş}

Regional Policies: Convergence, Trade, and the Allocation of Public Capital MGSoG Dissertation Series, № 61

\section{Florian Henning}

Living Up to Standard:

Interoperability Governance and

Standards Adoption in Government

Information Networks

MGSoG Dissertation Series, № 60

\section{Niels P. Groen}

The Never-Ending Project

Understanding E-Government Project

Escalation

MGSoG Dissertation Series, № 59

\section{Derek Copp}

Teacher-Based Reactivity to Provincial

Large-scale Assessment in Canada

MGSoG Dissertation Series, № 58 


\section{Michaella Vanore}

Family-Member Migration and the

Psychosocial Health Outcomes of

Children in Moldova and Georgia

MGSoG Dissertation Series, № 57

\section{Sonja Fransen}

The Economic and Social Effects of

Remittances and Return Migration in

Conflict-Affected Areas: The Case of

Burundi

MGSoG Dissertation Series, № 56

\section{Ibrahim Khalil Conteh}

The Impact of Floods on Primary

School Education in Zambia

MGSoG Dissertation Series, № 55

\section{Richard Bluhm}

Growth Dynamics and Development

Essays in Applied Econometrics and

Political Economy

MGSoG Dissertation Series, № 54

\section{Nevena P. Zhelyazkova}

Work-Family Reconciliation and Use of Parental Leave in Luxembourg:

Empirical Analysis of Administrative Records

MGSoG Dissertation Series, № 53

\section{4}

\section{Dirk Crass}

The Impact of Brands on Innovation and Firm Performance: Empirical Evidence from Germany UNU-MERIT Dissertation Series, № 87

\section{Samyukta Bhupatiraju}

The Geographic Dimensions of Growth and Development

UNU-MERIT Dissertation Series, № 86

\section{François Lafond}

The Evolution of Knowledge Systems UNU-MERIT Dissertation Series, № 85

\section{Annalisa Primi}

Promoting Innovation in Latin America: What Countries Have Learned (and What They Have Not) in Designing and Implementing Innovation and Intellectual Property Policies

UNU-MERIT Dissertation Series, № 84

\section{Fatoumata Lamarana Diallo}

Evaluation of Meal and Deworming

Programs for Primary Schools in Rural Senegal

UNU-MERIT Dissertation Series, № 83

\section{Sachin Kumar Badkas}

Metachoice and Metadata: Innovating with Environmental Policy Analysis in Europe

MGSoG Dissertation Series, № 52

\section{Irina S. Burlacu}

An Evaluation of Tax-Benefit Systems Impact on the Welfare of Frontier Worker:

The Case of Luxembourg and Belgium MGSoG Dissertation Series, № 51 


\section{Özge Bilgili}

Simultaneity in Transnational

Migration Research: Links Between

Migrants' Host and Home Country

Orientation

MGSoG Dissertation Series, № 50

\section{Yulia Privalova Krieger}

Reshaping the Big Agenda:

Transnational Politics and Domestic

Resistance Financial crisis and social

protection reform in Bosnia and

Herzegovina

MGSoG Dissertation Series, № 49

\section{Marieke van Houte}

Moving Back or Moving Forward?

Return migration after Conflict

MGSoG Dissertation Series, № 48

\section{Oxana Slobozhan}

Global Governance in the Management of Natural Resources: The Case of the Extractive Industries Transparency Initiative (EITI)

MGSoG Dissertation Series, № 47

\section{Luis Bernardo Mejia Guinand}

The Changing Role of the Central

Planning Offices in Latin America: A

Comparative Historical Analysis

Perspective (1950-2013)

MGSoG Dissertation Series, № 46

\section{Cheng Boon Ong}

Ethnic Segregation in Housing,

Schools and Neighbourhoods in the

Netherlands

MGSoG Dissertation Series, № 45

\section{Luciana V. Cingolani}

Bureaucracies for Development:

Oxymoron or Reality? Studies on State

Capacity in Challenging Governance

Contexts

MGSoG Dissertation Series, № 44

\section{Carlos Cadena Gaitán}

Green Politics in Latin American

Cities - Sustainable Transport Agendas

MGSoG Dissertation Series, № 43

\section{Katie Kuschminder}

Female Return Migration and

Reintegration Strategies in Ethiopia

MGSoG Dissertation Series, № 42

\section{Metka Hercog}

Highly-Skilled Migration and New

Destination Countries

MGSoG Dissertation Series, № 41

\section{Margaret Agaba Rugadya}

Can Remittances Influence the Tenure

and Quality of Housing in Uganda?

MGSoG Dissertation Series, № 40

\section{Ilire Agimi}

New Governance Under Limited

Statehood: The Case of Local

Government Reform in Kosovo

MGSoG Dissertation Series, № 39

\section{3}

\section{Anant Kamath}

Information Sharing through Informal Interaction in Low-Tech Clusters UNU-MERIT Dissertation Series, № 82 


\section{Flavia Pereira de Carvalho}

What we talk about when we talk about

Brazilian Multinationals: An

Investigation on Brazilian FDI,

Economic Structure, Innovation and

the Relationship between them

UNU-MERIT Dissertation Series, № 81

\section{Jun Hou}

Complementarity in Innovation and

Development: A Cross-country

Comparison

UNU-MERIT Dissertation Series, № 80

\section{Rufin Baghana}

Impacts of Government Incentives to $R \mathcal{E D}$, Innovation and Productivity:

A Microeconometric Analysis of the

Québec Case

UNU-MERIT Dissertation Series, № 79

\section{Lilia I. Stubrin}

High-Tech Activities in Emerging

Countries: A Network perspective on

the Argentinean Biotech Activity

UNU-MERIT/MGSoG Dissertation

Series, № 78

\section{Kristine Farla}

Empirical Studies on Institutions,

Policies and Economic Development

MGSoG Dissertation Series, № 38

\section{Marina Petrovic}

Social Assistance and Activation in the Pursuit of Happiness: Shedding New

Light on Old Policy Solutions to Social Exclusion

MGSoG Dissertation Series, № 37

\section{Laura Torvinen}

Assessing Governance Assessments:

The Case of Mozambique: Governance

Assessments in the Context of Aid

Effectiveness Discourse

MGSoG Dissertation Series, № 36

\section{Biniam Egu Bedasso}

Institutional Change in the Long

Shadow of Elite: Essays on

Institutions, Human Capital and

Ethnicity in Developing Countries

MGSoG Dissertation Series, № 35

\section{Sepideh Yousefzadeh Faal}

Deghati

Childhoods Embargoed: Constructing and Reconstructing Multidimensional

Child Poverty in Iran 1984-2009

MGSoG Dissertation Series, № 34

\section{Robert Bauchmüller}

Investing in Early Childhood Care and Education: The Impact of Quality on Inequality

MGSoG Dissertation Series, № 33

\section{Martin Rehm}

Unified Yet Separated: Empirical

Study on the Impact of Hierarchical

Positions within Communities of

Learning

MGSoG Dissertation Series, № 32 


\author{
Abdul Waheed \\ Innovation Determinants and \\ Innovation as a Determinant: Evidence \\ from Developing Countries \\ UNU-MERIT Dissertation Series, \\ № 77
}

\section{Bilal Mirza}

Energy Poverty and Rural Energy

Markets in Pakistan

UNU-MERIT Dissertation Series, № 76

\section{Benjamin Engelstätter}

Enterprise Software and Video Games:

An Empirical Analysis

UNU-MERIT Dissertation Series, № 75

\section{Fulvia Farinelli}

Natural Resources, Innovation and

Export Growth: The Wine Industry in

Chili and Argentina

UNU-MERIT Dissertation Series

\section{Rodolfo Lauterbach}

Innovation in Manufacturing: From

Product Variety and Labor

Productivity Growth to Economic

Development in Chile

UNU-MERIT Dissertation Series

\section{Kirsten Wiebe}

Quantitative Assessment of

Sustainable Development and Growth

in Sub-Saharan Africa

UNU-MERIT Dissertation Series, № 74
Julio Miguel Rosa

Organizational Strategies, Firms'

Performance and Spatial Spillovers:

The Canadian Case in Research and

Development.

UNU-MERIT Dissertation Series, № 73

Johannes Wilhelmus Marie Boels

Joseph Schumpeter, Honderd Jaar

Economische Ontwikkeling: Een

Historisch-theoretische Beschouwing.

UNU-MERIT Dissertation Series

\section{Dorcas Mbuvi}

Utility Reforms and Performance of the

Urban Water Sector in Africa

MGSoG Dissertation Series, № 31

\section{Lina Salanauskaite}

Distributional Impacts of Public

Policies: Essays in Ex-Ante and Ex-

Post Evaluation

MGSoG Dissertation Series, № 30

\section{Esther Schüring}

To Condition or not - is that the

Question?

An Analysis of the Effectiveness of Ex-

Ante and Ex-Post Conditionality in

Social Cash Transfer Programs

MGSoG Dissertation Series, № 29

\section{Joe Abah}

Strong Organisations in Weak States:

Atypical Public Sector Performance in

Dysfunctional Environments

MGSoG Dissertation Series, № 28 


\section{Zina Samih Nimeh}

Social Citizenship Rights: Inequality and Exclusion

MGSoG Dissertation Series, № 27

2011

\section{Daniel Vertesy}

Interrupted Innovation: Emerging

Economies in the Structure of the

Global Aerospace Industry

UNU-MERIT Dissertation Series, № 72

\section{Tina Saebi}

Successfully Managing Alliance

Portfolios: An Alliance Capability

View

UNU-MERIT Dissertation Series, № 71

\section{Nora Engel}

Tuberculosis in India: A Case of Innovation and Control

UNU-MERIT/MGSoG Dissertation

Series, № 70

\section{Evans Mupela}

Connectivity and growth in Sub-

Saharan Africa: The Role of

Communication Satellites

UNU-MERIT Dissertation Series, № 69

\section{Nantawan Kwanjai}

Cross Cultural Intelligence amid

Intricate Cultural Webs: A Tale of the

UnDutchables in the Land of 1002

Smiles

UNU-MERIT Dissertation Series, № 68

\section{Lina Sonne}

Innovation in Finance to Finance Innovation: Supporting Pro-poor Entrepreneur-based Innovation UNU-MERIT Dissertation Series, № 67

\section{Lenka Eisenhamerová}

Legitimacy of 'Humanitarian Military Intervention'

MGSoG Dissertation Series, № 26

\section{Sonila Tomini}

Informal Payments for Health Care

Services in Albania

MGSoG Dissertation Series, № 25

\section{Jinjing Li}

Dynamic Microsimulation in Public

Policy Evaluation

MGSoG Dissertation Series, № 24

\section{Aziz Atamanov}

Rural Nonfarm Employment and International Migration as

Alternatives to Agricultural

Employment: The Case of Kyrgyzstan

MGSoG Dissertation Series, № 23

\section{Frieda Vandeninden}

Poverty Alleviation: Aid and Social

Pensions

MGSoG Dissertation Series, № 22

\section{Juliana Nyasha Tirivayi}

The Welfare Effects of Integrating AIDS Treatment with Food Transfers: Evidence from Zambia

MGSoG Dissertation Series, № 21 
Agnieska Ewa Sowa

Who's Left Behind? Social Dimensions

of Health Transition and Utilization of

Medical Care in Poland

MGSoG Dissertation Series, № 20

\section{Emmanaouil Sfakianakis}

The Role of Private Actors in the

Provision of Public Goods with

Applications to Infrastructure and

Financial Stability

MGSoG Dissertation Series, № 19

\section{Siu Hing Lo}

White Collars Green Sleeves: An Inter-

organizational Comparison of

Determinants of Energy-Related

Behaviors among Office Workers

MGSoG Dissertation Series, № 18

\section{Treena $\mathbf{W u}$}

Constraints to Human Capital

Investment in Developing Countries:

Using the Asian Financial Crisis in

Indonesia as a Natural Experiment

MGSoG Dissertation Series, № 17

\section{Henry Espinoza Peña}

Impact Evaluation of a Job-Training

Programme for Disadvantaged Youths:

The Case of Projoven

MGSoG Dissertation Series, № 16
2010

\section{Fernando Santiago}

Human Resources Management

Practices and Learning for Innovation in Developing Countries:

Pharmaceutical Firms in Mexico

UNU-MERIT Dissertation Series,

№ 66

\section{Zakaria Babutsidze}

Essays on Economies with

Heterogeneous Interacting Consumers

UNU-MERIT Dissertation Series,

№ 65

\section{Bertha Vallejo}

Learning and Innovation Under

Changing Market Conditions: The

Auto Parts Industry in Mexico

UNU-MERIT Dissertation Series,

№ 64

\section{Donatus Ayitey}

Technical Change, Competitiveness

and Poverty Reduction: A Study of the

Ghanaian Apparel Industry

UNU-MERIT Dissertation Series, № 63

\section{Sergey Filippov}

Multinational Subsidiary Evolution:

Corporate Change in New EU Member

States

UNU-MERIT Dissertation Series, № 62 


\section{Asel Doranova}

Technology Transfer and Learning under the Kyoto Regime: Exploring the Technological Impact of CDM Projects in Developing Countries

UNU-MERIT Dissertation Series, № 61

\section{Florian Tomini}

Between Family and Friend:

Understanding the Interdependency of Private Transfers

MGSoG Dissertation Series, № 15

\section{Michał Polalowski}

The Institutional Transformation of

Social Policy in East Central Europe:

Poland and Hungary in Comparative and Historical Perspective

MGSoG Dissertation Series, № 14

\section{Maha Ahmed}

Defining, Measuring and Addressing Vulnerability: The Case of Post

Conflict Environments

MGSoG Dissertation Series, № 13

\section{Pascal Beckers}

Local Space and Economic Success:

The Role of Spatial Segregation of

Migrants in the Netherlands

MGSoG Dissertation Series, № 12

\section{Victor Cebotari}

Conflicting Demands in Ethnically

Diverse Societies: Ethno political

Contention and Identity Values in

Europe

MGSoG Dissertation Series, № 11

\section{Dennis Gyllensporre}

Competing and Complementary

Perspectives on the EU as a Crisis

Management Actor:

An Examination of the Common

Security and Defence Policy through

the Lenses of Idealism and Realism

MGSoG Dissertation Series, № 10

\section{Judit Vall Castello}

Business Cycle and Policy Effects on Labour Market Transitions of Older and Disabled Workers in Spain

MGSoG Dissertation Series, № 9

\section{Keetie Roelen}

False Positives or Hidden Dimensions:

The Definition and Measurement of

Child Poverty

MGSoG Dissertation Series, № 8

Denisa Maria Sologon

Earning Dynamics in Europe

MGSoG Dissertation Series, № 7

\section{Melissa Siegel}

Money and Mobility: Migration and

Remittances

MGSoG Dissertation Series, № 6

Jessica S. Hagen-Zanker

Modest Expectations: Causes and

Effects of Migration on Migrant

Households inSource Countries

MGSoG Dissertation Series, № 5 
Alexis Habiyaremye

From Primary Commodity Dependence to Diversification and Growth:

Absorptive Capacity and Technological Catch Up in Botswana and Mauritius. UNU-MERIT Dissertation Series, № 60

\section{Yoseph Getachew}

The Role of Public Capital in Economic Development

UNU-MERIT Dissertation Series, № 59

\section{Sandra Leitner}

Embodied Technological Change and Patterns of Investment in Austrian Manufacturing UNU-MERIT Dissertation Series, № 58

\section{Semih Akçomak}

The Impact of Social Capital on Economic and Social Outcomes UNU-MERIT Dissertation Series, № 57

\section{Abraham Garcia \\ The Role of Demand in Technical Change \\ UNU-MERIT Dissertation Series, № 56}

\section{Saurabh Arora}

Coherence in Socio-technical Systems: A Network Perspective on the Innovation Process UNU-MERIT Dissertation Series, № 55
Human Development and Autonomy in Project Aid: Experiences from four bilateral projects in Nicaragua and $E l$ Salvador

MGSoG Dissertation Series, № 4

\section{Christiane Arndt}

Governance Indicators

MGSoG Dissertation Series, № 3

\section{Britta Augsburg}

Microfinance: Greater Good or Lesser Evil?

MGSoG Dissertation Series, № 2

\section{8}

\section{Rutger Daems}

Medicines for the Developing World UNU-MERIT Dissertation Series, № 54

\section{Johannes Hanel}

Assessing Induced Technology:

Sombart's Understanding of Technical

Change in the History of Economics

UNU-MERIT Dissertation Series, № 53

\section{Rifka Weehuizen}

Mental Capital: the Economic Significance of Mental Health UNU-MERIT Dissertation Series, № 52 


\section{Danielle Cloodt}

The Relationship between RED

Partnership Formation, Social

Embeddedness and Innovative

Performance

UNU-MERIT Dissertation Series, № 51

\section{Sabine Fuss}

Sustainable Energy Development

under Uncertainty

UNU-MERIT Dissertation Series, № 50

\section{Geranda Notten}

Measuring and Managing Poverty

Risks

MGSoG Dissertation Series, № 1

2007

\section{Tobias Kronenberg}

Reconciling Environmental

Conservation with Economic

Prosperity: The Feasibility of Double

Dividends in the Short and Long Run

UNU-MERIT Dissertation Series, № 49

\section{Viktoria Kravtsova}

Assessing the Impact of Foreign Direct Investment in Transition Economies UNU-MERIT Dissertation Series, № 48

\section{Suhail Sultan}

The Competitive Advantage of Small and Medium Sized Enterprises: The Case of Jordan's Natural Stone Industry UNU-MERIT Dissertation Series, № 47

\section{6}

\section{Bulat Sanditov}

Essays on Social Learning and Imitation

UNU-MERIT Dissertation Series, № 46

\section{Mamata Parhi}

Dynamics of New Technology Diffusion: A Study of the Indian Automotive Industry

UNU-MERIT Dissertation Series, № 45

\section{Andreas Reinstaller}

Social Structures and the Innovation Process: Their Role in the Demand of Firms and Consumers

UNU-MERIT Dissertation Series, № 44

\section{Rose Kiggundu}

Innovation systems and Development: The Journey of a Beleaguered Nile Perch Fishery in Uganda UNU-MERIT Dissertation Series, № 43 


\section{Thomas Pogue}

The Evolution of Research

Collaboration in South African Gold

Mining: 1886-1933

UNU-MERIT Dissertation Series, № 42

\section{Geoffrey Gachino}

Foreign Direct Investment, Spillovers and Innovation: The Case of Kenyan

Manufacturing Industry

UNU-MERIT Dissertation Series, № 41

\section{Önder Nomaler}

Technological Change, International

Trade and Growth: An Evolutionary,

Multi-Agents-Based Modeling

Approach

UNU-MERIT Dissertation Series, № 40

2005

Samia Satti Osman Mohamed-

Nour

Change and Skill Development in the Arab Gulf Countries

UNU-MERIT Dissertation Series, № 39

\section{Elad Harison}

Intellectual Property Rights:

Economics and Policy Analysis

UNU-MERIT Dissertation Series, № 38

\section{Daniel Dalohoun}

Learning to innovate: agricultural innovation and entrepreneurship: the case of Songhai farmers in Benin UNU-MERIT Dissertation Series, № 37

\section{Müge Ozman}

Networks, Organizations and

Knowledge

UNU-MERIT Dissertation Series, № 36

\section{Bas Straathof}

Product Variety and Economic

Growth: The Counteracting Effects of

Scale and Idiosyncrasy

UNU-MERIT Dissertation Series, № 35

\section{Wilfred Schoenmakers}

Knowledge Flows between

Multinational Companies: A Patent

Data Analysis

UNU-MERIT Dissertation Series, № 34

\section{Myriam Cloodt}

Mergers and Acquisitions ( $M$ and As) in High-Tech Industries: Measuring the Post-M and A Innovative Performance of Companies UNU-MERIT Dissertation Series, № 33 
2004

\section{Paola Criscuolo}

$R \mathcal{E D}$ Internationalisation and

Knowledge Transfer: Impact on MNEs and their Home Countries

UNU-MERIT Dissertation Series, № 32

\section{Maarten Verkerk}

Trust and Power on the Shop Floor UNU-MERIT Dissertation Series, № 31

\section{Gottfried Leibbrandt}

Adoption, Harmonization and Succession of Network Technologies across Countries

UNU-MERIT Dissertation Series, № 30

\section{Mark Sanders}

Skill Biased Technical change: Its Origins, the Interaction with the Labour Market and Policy Implications UNU-MERIT Dissertation Series, № 29

2003

\section{Nadine Roijakkers}

Inter-firm Cooperation in High-tech Industries: a Study of RED

Partnerships in Pharmaceutical

Biotechnology

UNU-MERIT Dissertation Series, № 28
Viki Sonntag

Speed, Scale and Sustainability

UNU-MERIT Dissertation Series, № 27

\section{Masaru Yarime}

From End-of-Pipe Technology to Clean Technology

UNU-MERIT Dissertation Series, № 26

\section{Stéphane Malo}

The Combinatorial Chemistry

Revolution: Sustaining a Superior

Performance Position through

Technological Learning

UNU-MERIT Dissertation Series, № 25

2002

\section{Annelies Hogenbirk}

Determinants of Inward Foreign Direct Investment: the Case of the Netherlands

UNU-MERIT Dissertation Series, № 24

\section{Bastiaan Johan terWeel}

The Computerization of the Labour Market

UNU-MERIT Dissertation Series 


\section{Marjolein Caniëls}

\section{John Adeoti}

Technology Investment in Pollution Control in Sub-Saharan Africa: The Case of the Nigerian Manufacturing Industry

UNU-MERIT Dissertation Series, № 23

\section{Edward Huizenga}

Innovation Management: How

Frontrunners Stay Ahead: An

Empirical Study on Key Success

Factors in the ICT sector

UNU-MERIT Dissertation Series, № 22

2000

\section{Machiel van Dijk}

Technological Change and the

Dynamics of Industries: Theoretical Issues and Empirical evidence from

Dutch Manufacturing

UNU-MERIT Dissertation Series, № 21

1999

\section{Jan Cobbenhagen}

Managing Innovation at the Company Level: A Study on Non-Sector-Specific Success Factors UNU-MERIT Dissertation Series, № 20
Regional Growth Differentials: The Impact of Locally Bounded Knowledge Spillovers

UNU-MERIT Dissertation Series, № 19

\section{8}

\author{
Aldo Geuna \\ Resource Allocation and Knowledge \\ production: Studies in the Economics \\ of University Research \\ UNU-MERIT Dissertation Series, \\ № 18 \\ 1996

\section{Reinoud Joosten} \\ Dynamics, Equilibria, and Values \\ UNU-MERIT Dissertation Series, \\ № 17
}

\section{Hugo Kruiniger}

Investment, $R \mathcal{E} D$, and the Financing Decisions of the Firm

UNU-MERIT Dissertation Series, № 16

\section{5}

\section{Hans van Meijl}

Endogenous Technological Change: The Case of Information Technology, Theoretical Considerations and Empirical Results UNU-MERIT Dissertation Series, № 15 


\section{René Kemp}

Environmental Policy and Technical

Change: A Comparison of the

Technological Impact of Policy

Instruments

UNU-MERIT Dissertation Series, № 14

\section{Rohini Acharya}

The Impact of New Technologies on

Economic Growth and Trade: A Case

Study of Biotechnology

UNU-MERIT Dissertation Series, № 13

\section{Geert Duysters}

The Evolution of Complex Industrial Systems: The Dynamics of Major IT Sectors

UNU-MERIT Dissertation Series, № 12

\section{Marjan Groen}

Technology, Work and Organisation: A Study of the Nursing Process in Intensive Care Units

UNU-MERIT Dissertation Series, № 11

1994

\section{Huub Meijers}

On the Diffusion of Technologies in a Vintage Framework: Theoretical Considerations and Empirical Results UNU-MERIT Dissertation Series, № 10

\section{Theon van Dijk}

The Limits of Patent Protection: Essays on the Economics of Intellectual

Property Rights

UNU-MERIT Dissertation Series, № 9

\section{Hans Voordijk}

Naar Integrale Logistiek in

Bedrijfsketens: Ontwikkelingen in de

Bouw

UNU-MERIT Dissertation Series, № 8

1993

\section{Paul Diederen}

Technological Progress in Enterprises and Diffusion of Innovation:

Theoretical Reflections and Empirical Evidence

UNU-MERIT Dissertation Series, № 7

\section{Ben Dankbaar}

Economic Crisis and Institutional Change: The Crisis of Fordism from the Perspective of the Automobile Industry UNU-MERIT Dissertation Series, № 6

\section{Hanno Roberts}

Accountability and Responsibility: The Influence of Organisation Design on Management Accounting UNU-MERIT Dissertation Series, № 5 


\section{Bart Verspagen}

Uneven Growth between

Interdependent Economies: An

Evolutionary View on Technology

Gaps, Trade and Growth

UNU-MERIT Dissertation Series,

№ 4

\section{Sjoerd Romme}

A Self-organization Perspective on

Strategy Formation

UNU-MERIT Dissertation Series,

№ 3

1989

John Spangenberg

Economies of Scale, and Atmosphere in

Research Organisations

UNU-MERIT Dissertation Series,

№ 2

1988

John Hagedoorn

Evolutionary and Heterodox

Innovation Analysis: A Study of

Industrial and Technological

Development in Process Control and

Information Technology

UNU-MERIT Dissertation Series, № 1 